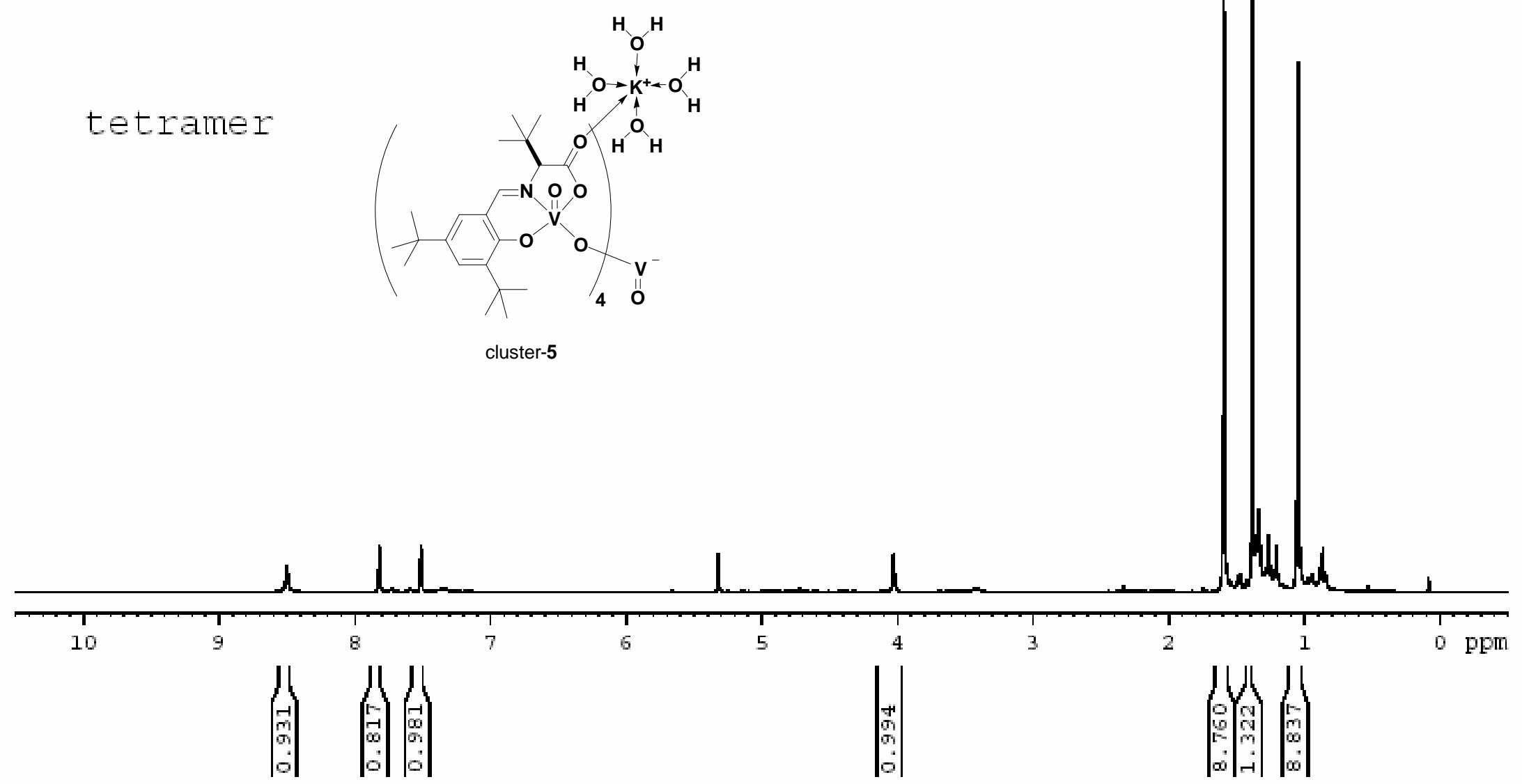




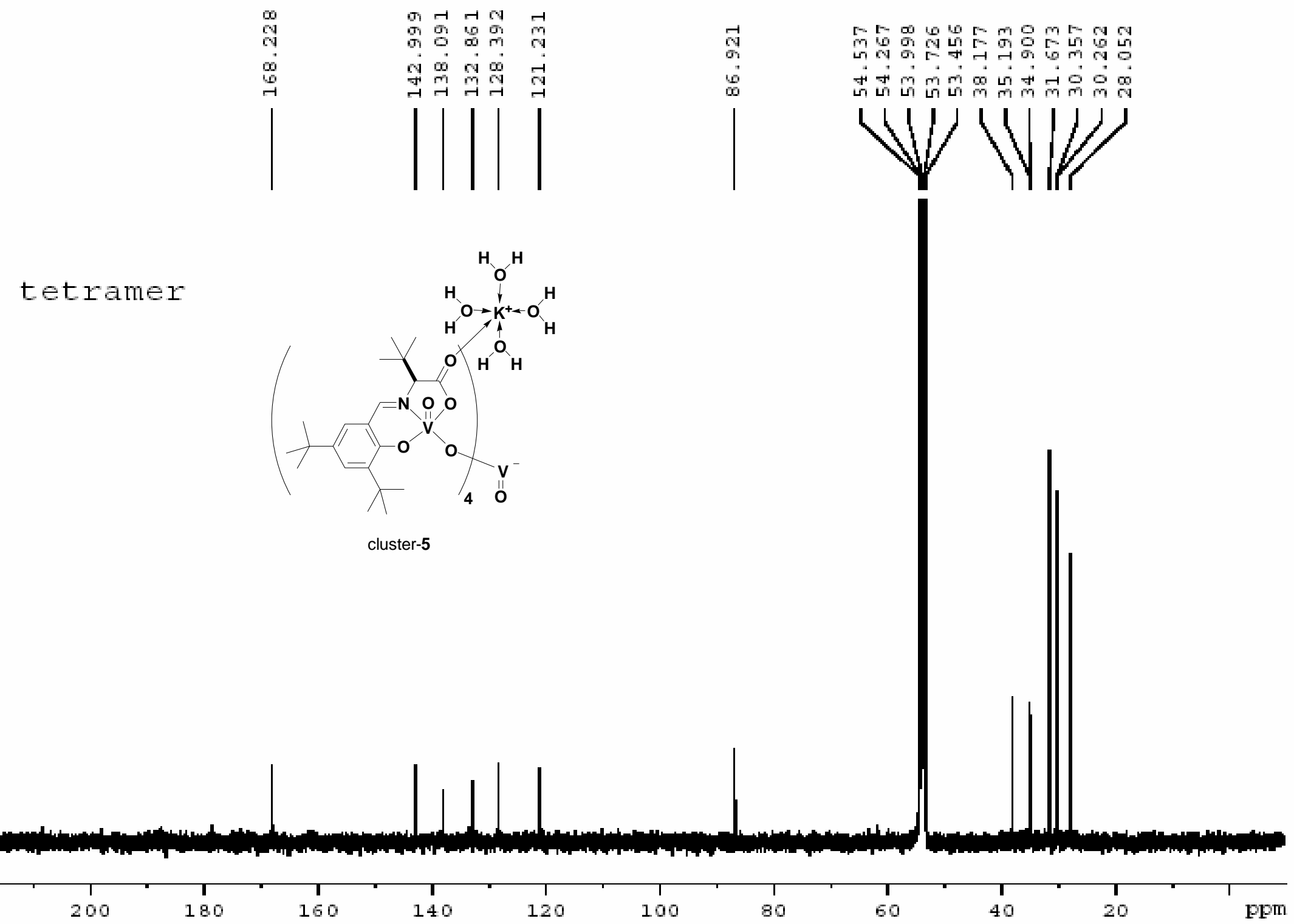




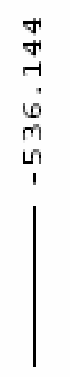

웅요

ตं

606

1

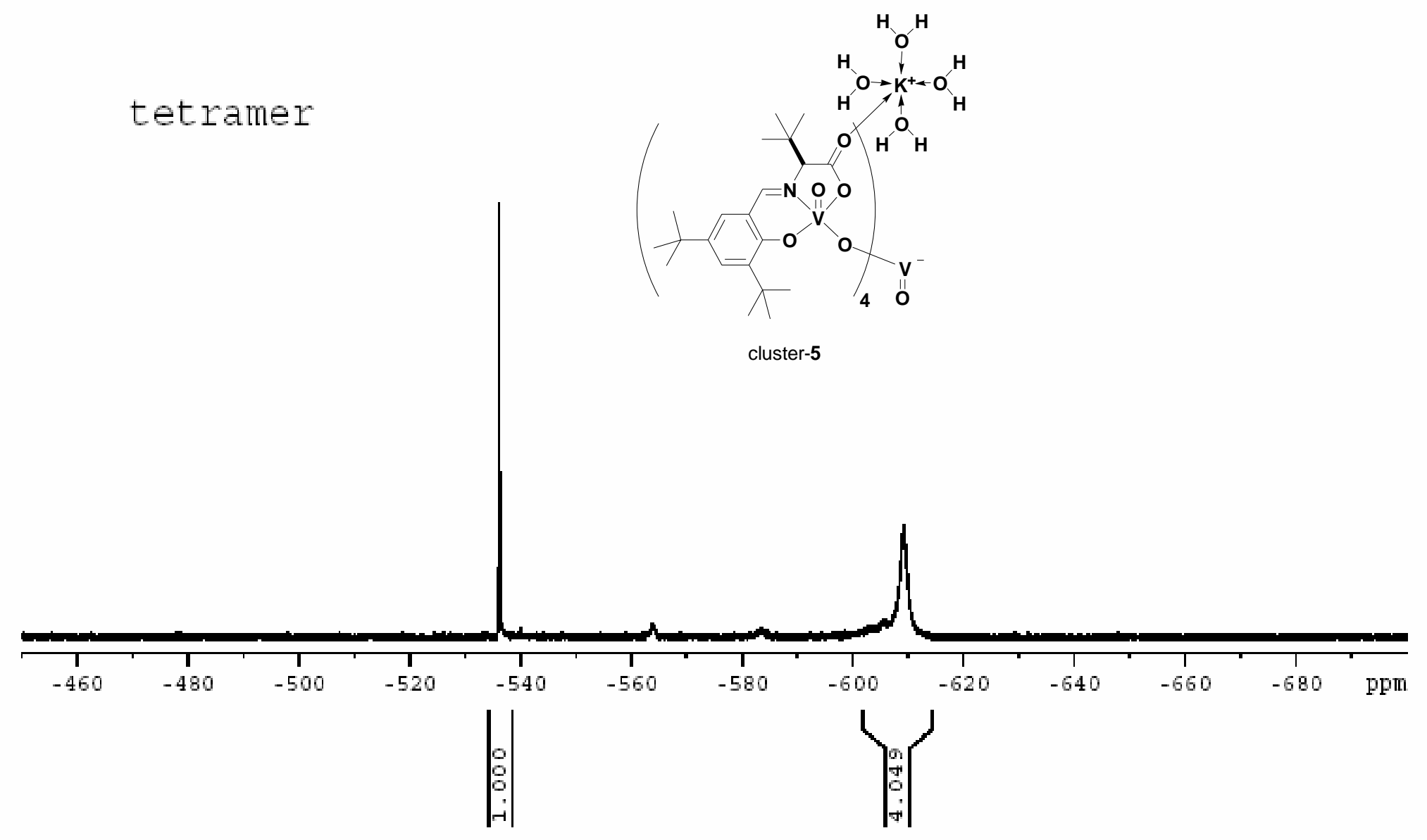


H spectrum of
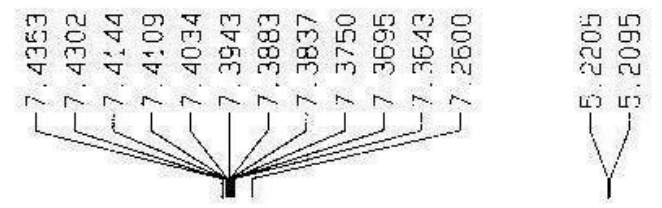

$\mathrm{OH}$ S

8

$8 a$
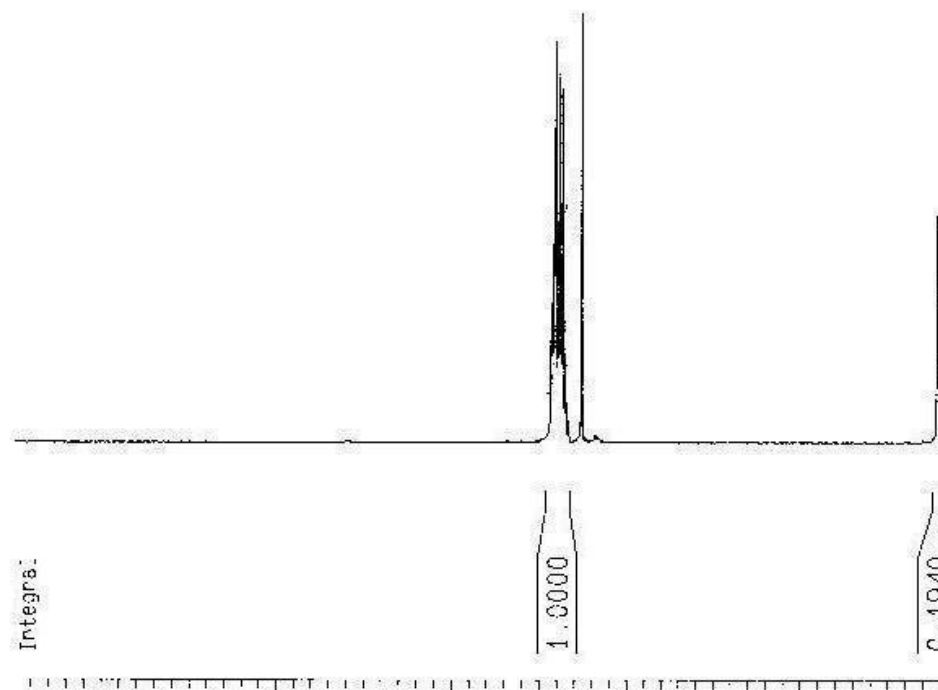

ppm 9

7

6

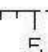

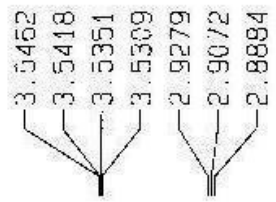
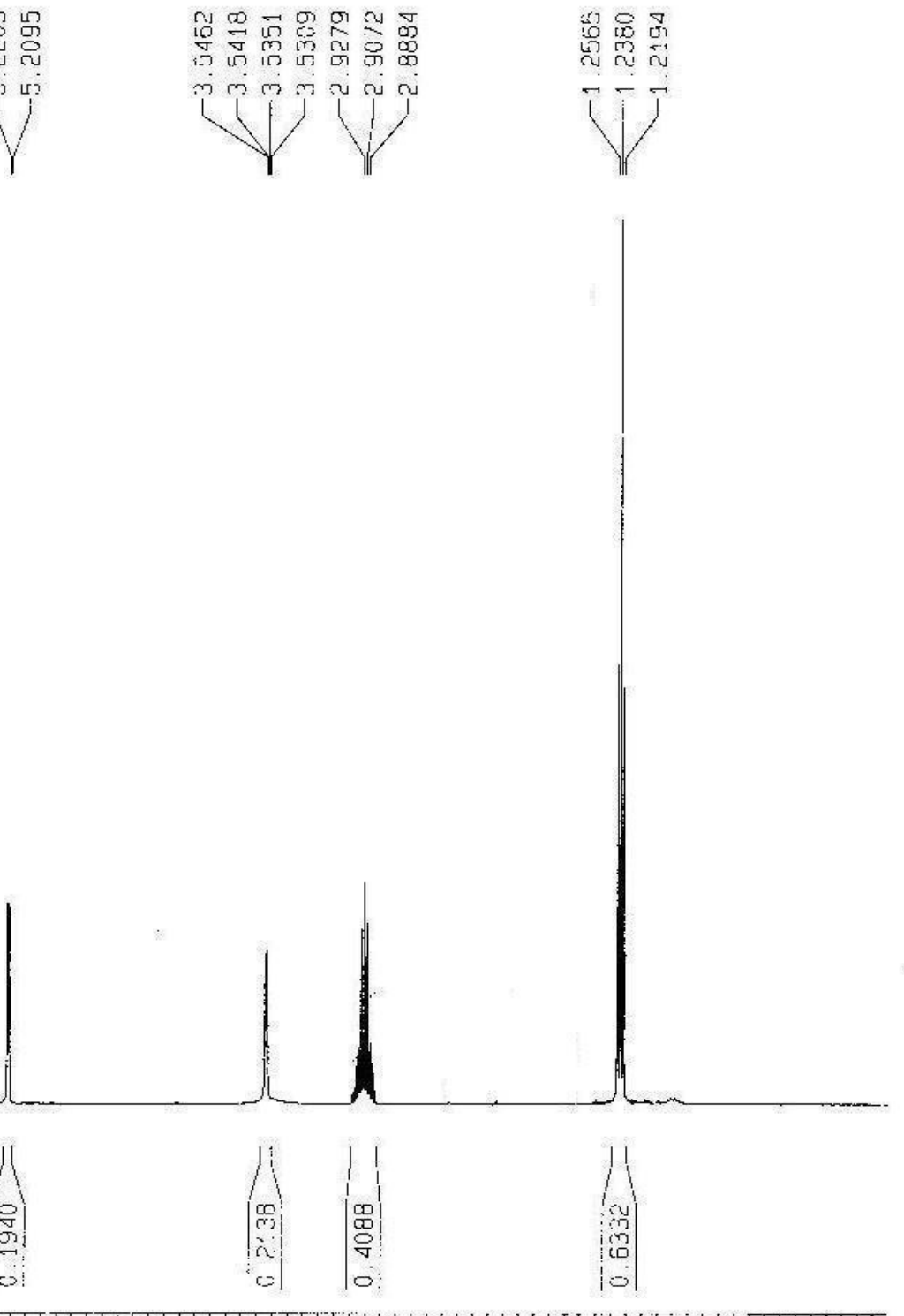

VAME

=XPNO
SROCNO

et.r thio est

=2 - Arquisitzor Parameters

jate_ 20051021

Time 20051021
$203 \%$

INSTRUKA SPREL

$5 \mathrm{~mm}$ BEO BB. $1 \mathrm{H}$

SOL WEN

5 WH

- IDHES $\quad 0.36591 \mathrm{~B} \mathrm{~Hz}$

$\Delta 0 \quad 1.3664756 \mathrm{ser}$

76

JW

TE

362
3600 use

6.50 user.

$300.0 \mathrm{~K}$

1. $50000000 \mathrm{sec}$

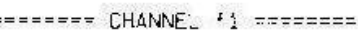

NUC1 $1 \mathrm{H}$

D. $1300 \mathrm{dE}$

SFC1 $\quad 400.132500 \mathrm{~B} M * 1 \mathrm{z}$

$=2$. Processang parameters

S1 $\quad 4001300095 \mathrm{MHz}$

HEW

Hew

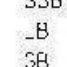

x

EM

$0 \quad 10 \mathrm{~Hz}$

15. AMR p]ot parameter's

$\begin{array}{rl}\mathrm{CY} & 20.00 \mathrm{~cm}\end{array}$

$\begin{array}{ll}C Y & 10.50 \mathrm{~cm}\end{array}$

$0.500 \mathrm{ppm}$
$\mathrm{Fi}$

$\begin{array}{ll}52 & 4201.37 \mathrm{~Hz}\end{array}$

$\begin{array}{ll}{ }^{5} & -0.500 \mathrm{DDm} \\ 5 & -200.07 \mathrm{~Hz}\end{array}$

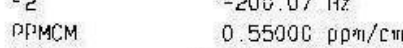

HZCM $220.07152 \mathrm{~Hz} / \mathrm{Cm}$ 
C13 scectrum cf

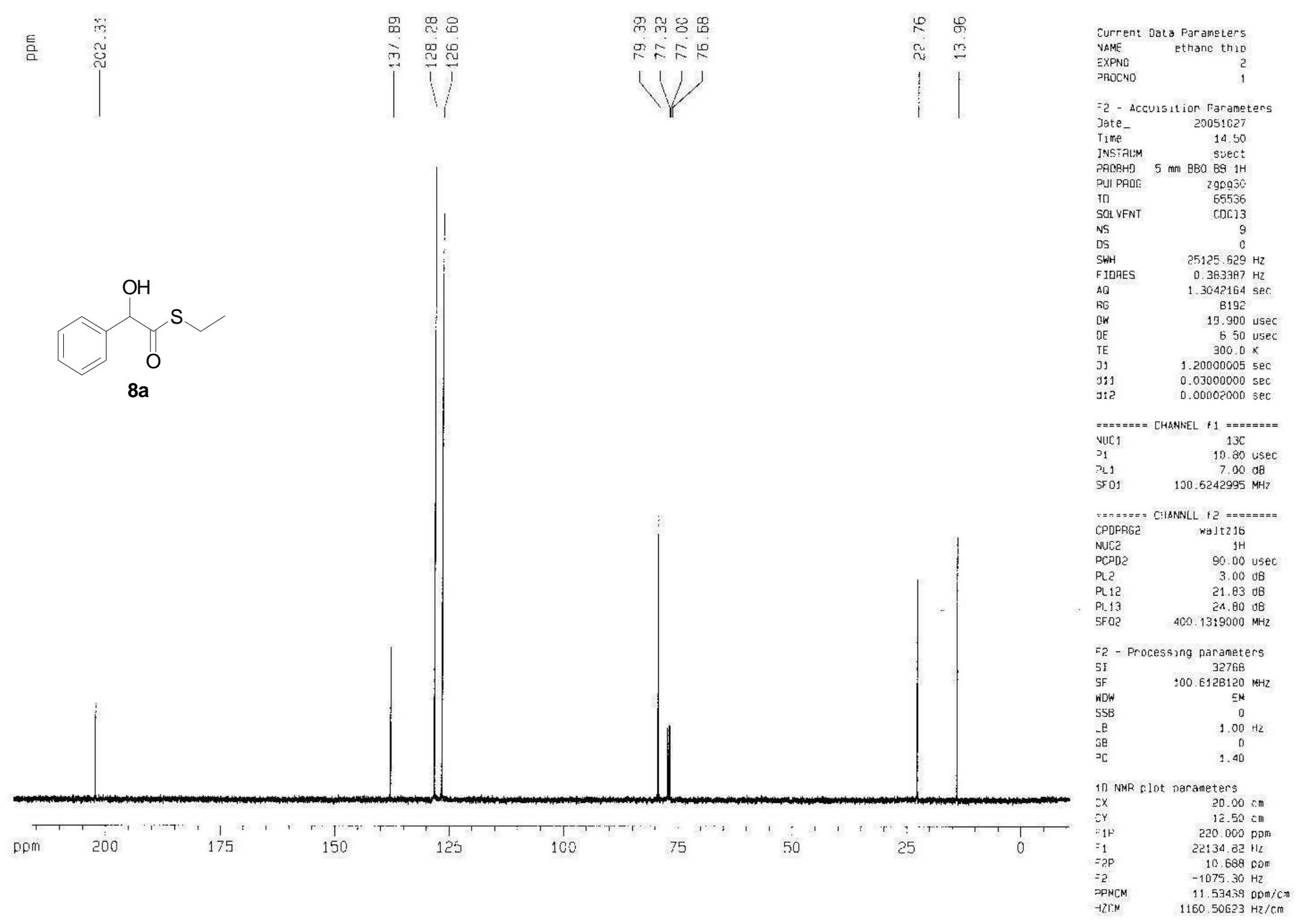


$H$ spectrum of

長
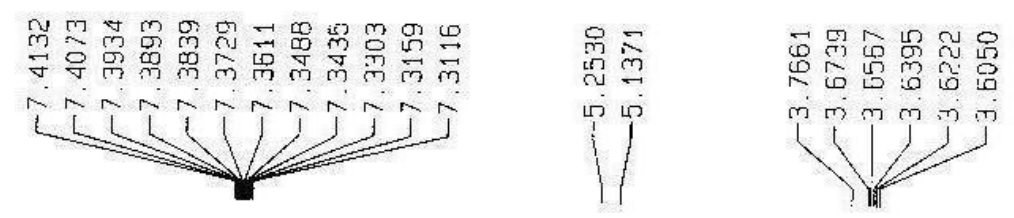

$\mathrm{OH}$

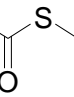

$8 b$

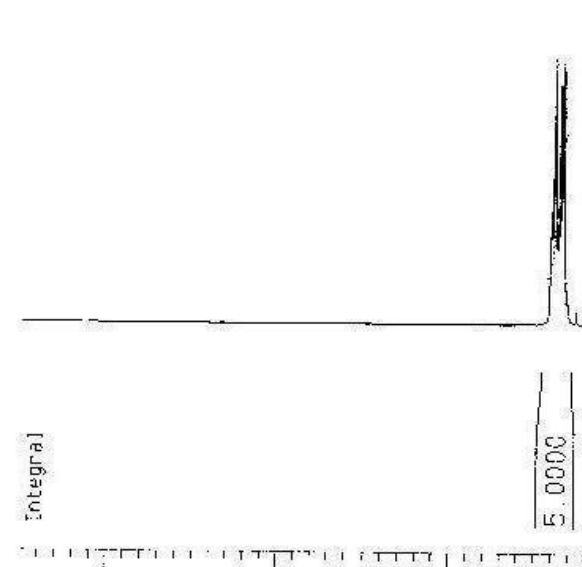

opm

g

8

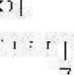

${ }^{+T T}+1:$ i ITT

6
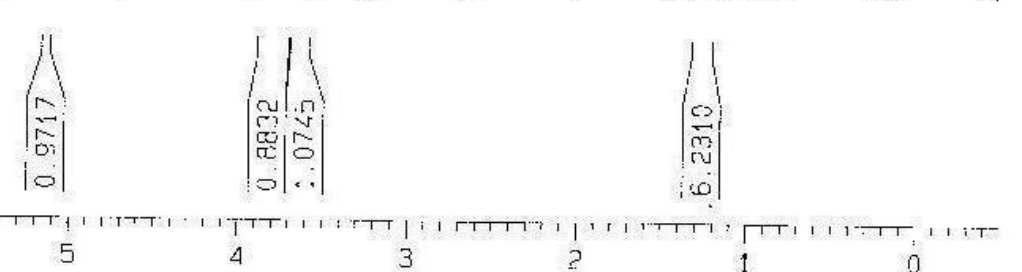

NAME

sam-150 HTi

PAOCNCS

ᄃa - AcLíisition Parameters

Date_ 200605?

T:me 16.07

Nhinum 5 spect

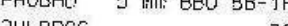

$\begin{array}{lr}\text { SULPFOG } & 2930 \\ \text { TO } & 16384\end{array}$

SOLVENT CDC13

NS

$\begin{array}{ll}35 & 0 \\ 5 \mathrm{WH} & 5995.204 \mathrm{~Hz}\end{array}$

FIDRES $\quad 5995.204 \mathrm{~Hz}$

4Q 1. $1.36 E 4756 \mathrm{SEC}$

$26 \quad 22.6$

DW $\quad 93.400$ use:

TE $\quad 6.50$ usec

$31 \quad 1.50000000 \mathrm{Kes}$

$===-===$ CHANNEL $+11==-====$

$==-===$ CHANNEL $+1 \quad=-====-$
$1 \mathrm{H}$

$\begin{array}{ll}\mathrm{P}_{1} & 1 \mathrm{H} \\ \mathrm{Nat} & 10.10 \mathrm{usec}\end{array}$

$\quad 400.1325000$ de

-2 - Prccesesıng garrämeters

SI 16384

WOW $\quad$ E.M

S.SB

$\begin{array}{rr}\mathrm{iB} & 0.10 \\ 3 \mathrm{~B} & 0\end{array}$

$\begin{array}{ll}x \\ x & 1.03\end{array}$

10 MMR plot paraneters

$\begin{array}{ll}Z X & 23.00 \mathrm{~cm} \\ Z Y & 10.50 \mathrm{~cm}\end{array}$

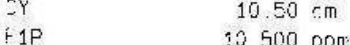
$420237 \mathrm{~Hz}$

$-0.500 \mathrm{pDm}$

$-200.07 \mathrm{~Hz}$

$0.55000 \mathrm{pem} / \mathrm{cm}$

$220.07152 \mathrm{~Hz} / \mathrm{cm}$ 


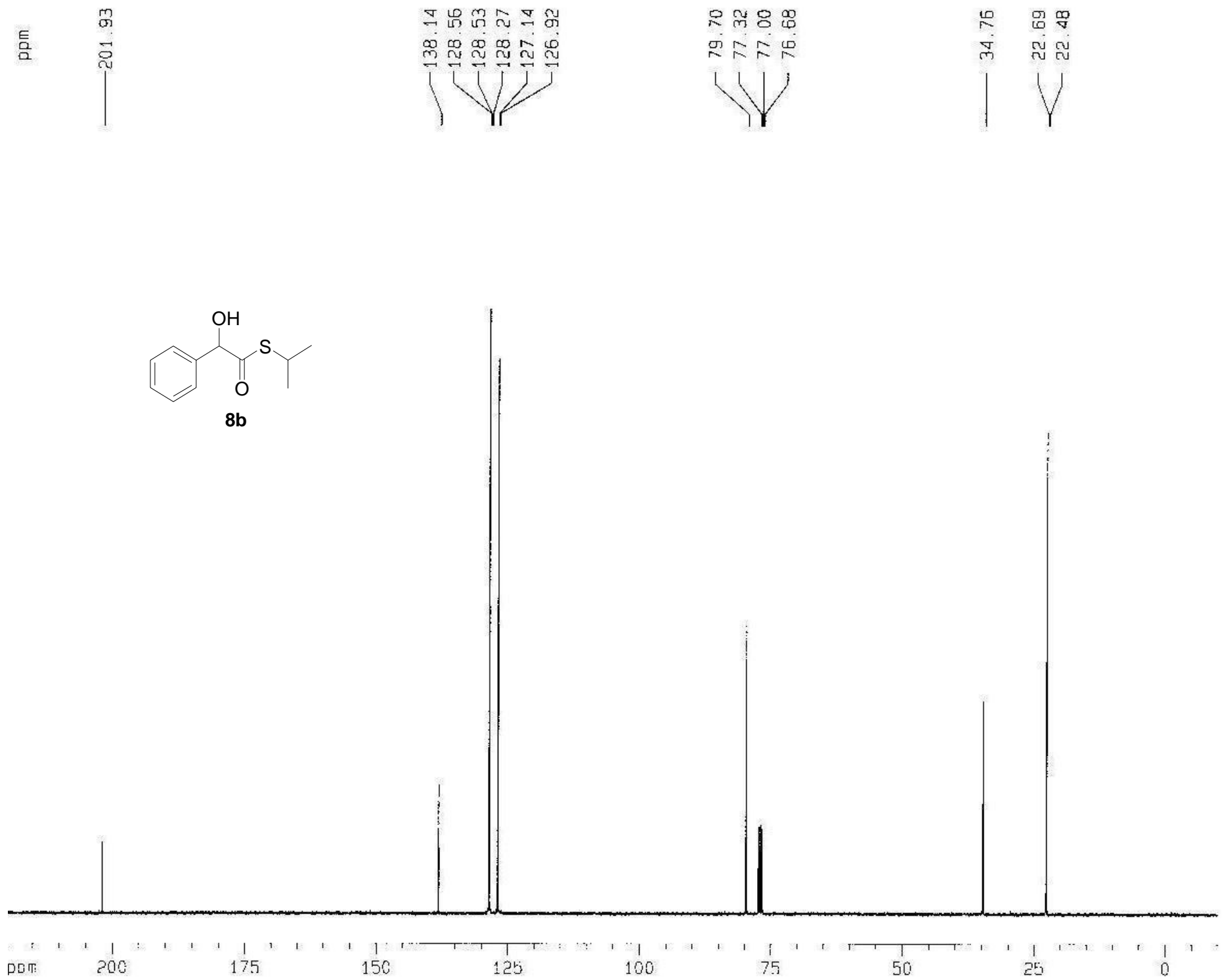

Current Data Parameters

SAM-150 HTI

=2 - Acquisjtigri Fanareter

Time- 16.14

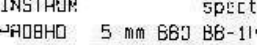

$29 p 530$

$\begin{array}{ll}\text { IU } & \text { E5535 } \\ \text { SO:VENT } & \text { CDC13 }\end{array}$

NS

इWWH $\quad 25125.623 \mathrm{~Hz}$

FIORES $\quad 0.393387 \mathrm{~Hz}$

AO $\quad 1.3042164 \mathrm{sec}$

1192

4.900 user

6. 50 usec

1. 200000055

$\quad 0.03000000 \mathrm{sec}$

$1120.00002000 \mathrm{sec}$

$======$ CHANWFL
NUCL
1.3.

$\mathrm{P}_{1} \quad 10.80$ usec

SFO1 1000242905 SH

CPOPHe

CPDPHGE waltz 16

NUC2 $\quad 90.00$

$\begin{array}{ll}\text { गCPO2 } & 90.00 \text { use } \\ \text { गL5 } & 3.00 \mathrm{~dB}\end{array}$

TL12 $21.83 \mathrm{dE}$

FFC? $400.1319000 \quad 452$

$=2$ - processing parameters

SI

$\begin{array}{ll}\text { SF } & 32750 \\ & 100.5127374 \mathrm{M} \\ \text { MIZ }\end{array}$

WDW

$\begin{array}{lc}S 59 & 2 \\ i .8 & 1.00 \mathrm{~Hz} \\ \mathrm{~GB} & 5\end{array}$

10] Nish plut paramelers

Cx $2000 \mathrm{~cm}$

$\begin{array}{lr}c r & 10.00 \mathrm{~cm} \\ \mathrm{cr} & 220.000 \mathrm{ppm}\end{array}$

$\begin{array}{ll}=1 & 22134.81 \mathrm{H} \% \\ =? \mathrm{P} & -10.002 \mathrm{~m}\end{array}$

$\begin{array}{lll}=2 & -10.000 \\ =2 & 00\end{array}$

गPMCM
IZCHA $11.50000 \mathrm{pPm} / \mathrm{Lm}$
$1157.04700 \mathrm{hz} / \mathrm{CT}$ 

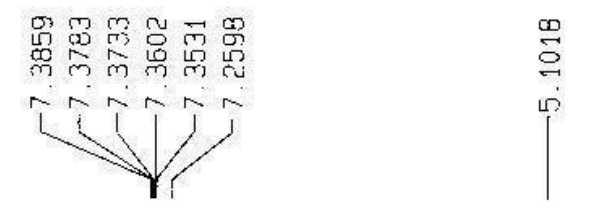

疍

i
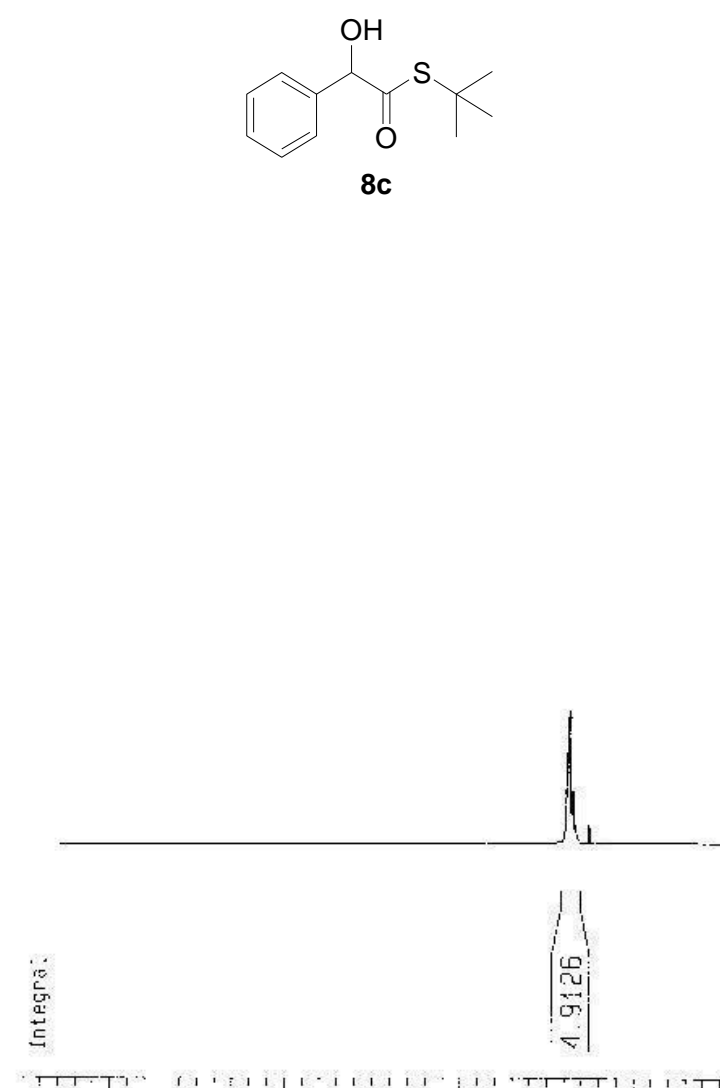

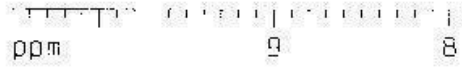

8

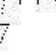

inther

5

5

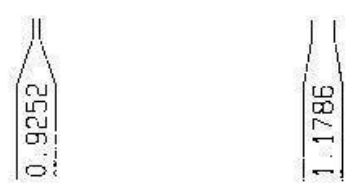

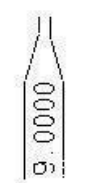

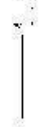

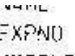

-2 - Acuulistion Parameters

Jate_ 200605z5

I I me $\quad 14.45$

ThOBD 5 an

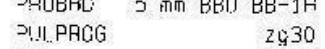

$\begin{array}{lr}\text { ग.II.PAGG } & 2430 \\ \text { TD } & \$ 6384\end{array}$

SQLVENT $\quad$ CUC:3

NS

as

CWH

-0.365918 Hz

40 $0.365918 \mathrm{~Hz}$

रह 1016

Ji' B.3.400 user

J5 6.50 use

TE
$\mathrm{D} 1.5000000 \mathrm{Kec}$

$=====--$ CHANNEL 11 ==:-"--+-

NUC.1 $1 \mathrm{H}$

$\begin{array}{ll}\mathrm{P}_{1} & 10.10 \mathrm{J5eC} \\ \mathrm{OL1} & 3.00 \mathrm{HB}\end{array}$

$100.32500 \mathrm{MHZ}$

-2 - Processing parameters

SI $163 \mathrm{EA}$

$\begin{array}{lc}\text { SF } & 400.1300391 \mathrm{MH}> \\ \text { WDW } & \text { EM }\end{array}$

WDW

$\therefore B \quad 0.10 \mathrm{~Hz}$

$\begin{array}{ll}\mathrm{SB} & 0 \\ \mathrm{OC} & 1.000\end{array}$

CD NMA Dlot parameters

Cx $\quad 20.60 \mathrm{~cm}$

Cy $\quad 0.50 \mathrm{~cm}$

=1P $10.500 \mathrm{FFm}$

$\begin{array}{ll}\tau_{1} & 4202.37 \mathrm{~Hz} \\ \tau ? \mathrm{~F} & -0.500 \mathrm{pgm}\end{array}$

$=2 \quad-200.07 \mathrm{~Hz}$

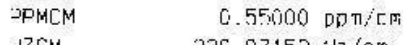

$-72 \mathrm{CM} \quad 22007152 \mathrm{~Hz} / \mathrm{cm}$ 


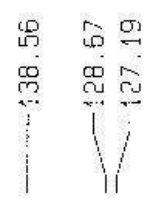

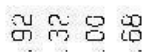

证点

11

$\mathrm{OH}$

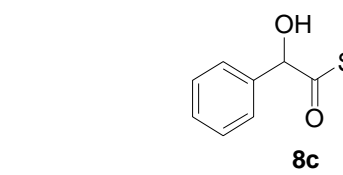

$8 c$

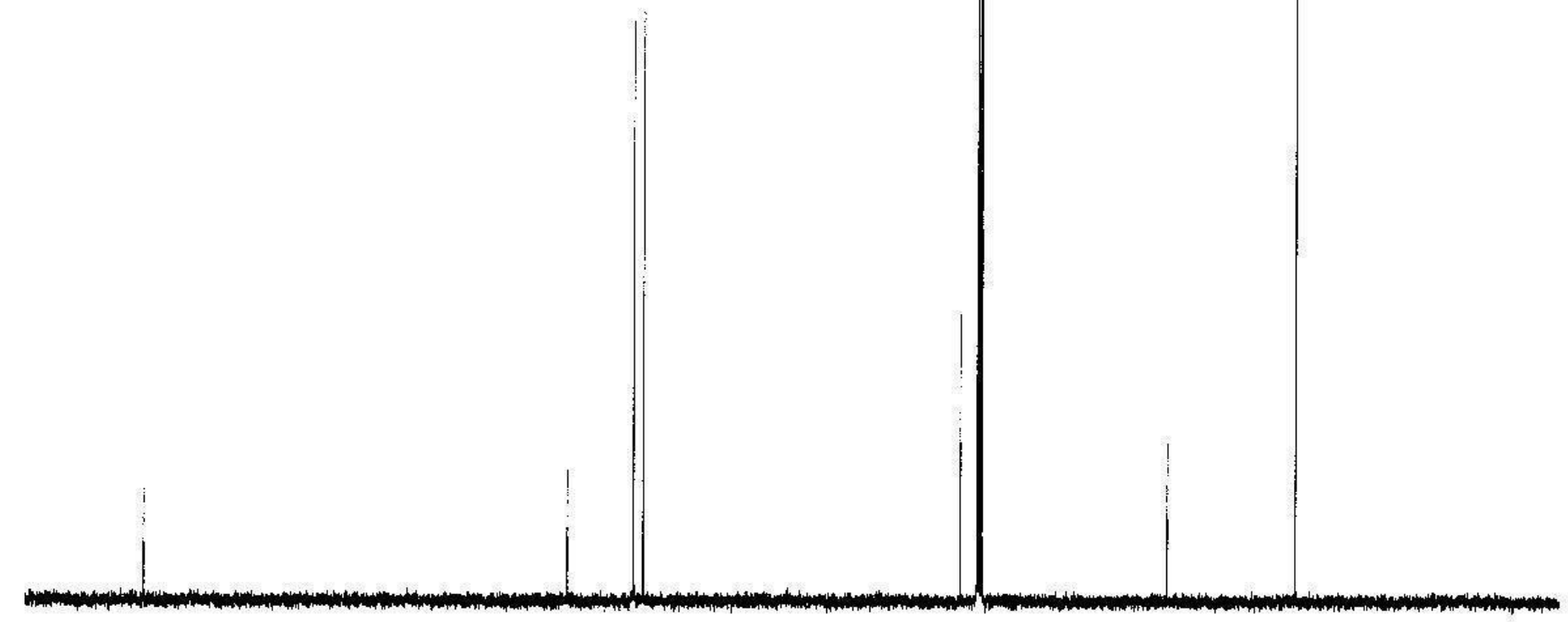

วpm
5000
500

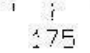

150

125

100

75

50

i

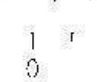

Curresit Uata Parmingters

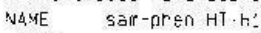

aRacNu

:2 - Arqujaition Parsneters

Tate _. 20EC5?

T:me

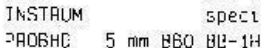

T9 $290 \mathrm{~g} 30$

$\begin{array}{lr}\text { SOLYEAT } & \text { COCT3 } \\ \text { VS } & \text { TRO }\end{array}$

5WH $\quad 25125.629 \mathrm{~Hz}$

0. $383387 \mathrm{~Hz}$

पढ

Jh 19.900 use.

$360.3 \mathrm{~K}$

1. Proonowos, sec

$0.0300000 \mathrm{sec}$
$5.0000200 \mathrm{sec}$

ale

$======$ CHANNEL
NUUC1 $1=====$
$13 \mathrm{C}$

$\mathrm{P}_{1} \quad 10 . \mathrm{BO} \mathrm{Usec}$

PL1 $\quad 7.00$ a日

$== \pm===$ CHANMEL if

EPUPAG 2HAT W3itz15

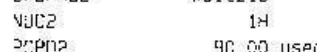

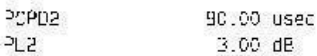

PL12 2:.83 de

LFil 400.1319000

$=2$ - Procossano parameters

$\begin{array}{ll}\mathrm{Si} & 32 / \mathrm{EE} \\ \mathrm{SF} & 200.6: 2 / 3 \mathrm{EH} M \mathrm{MH}\end{array}$

WCW
SSR

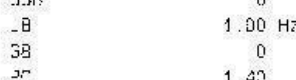

10 biMf plat. farameters

Cx $20.00 \mathrm{con}$

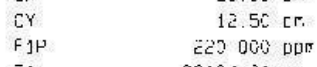

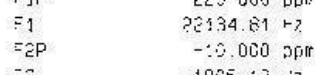

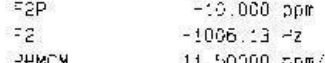

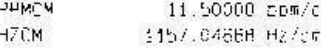



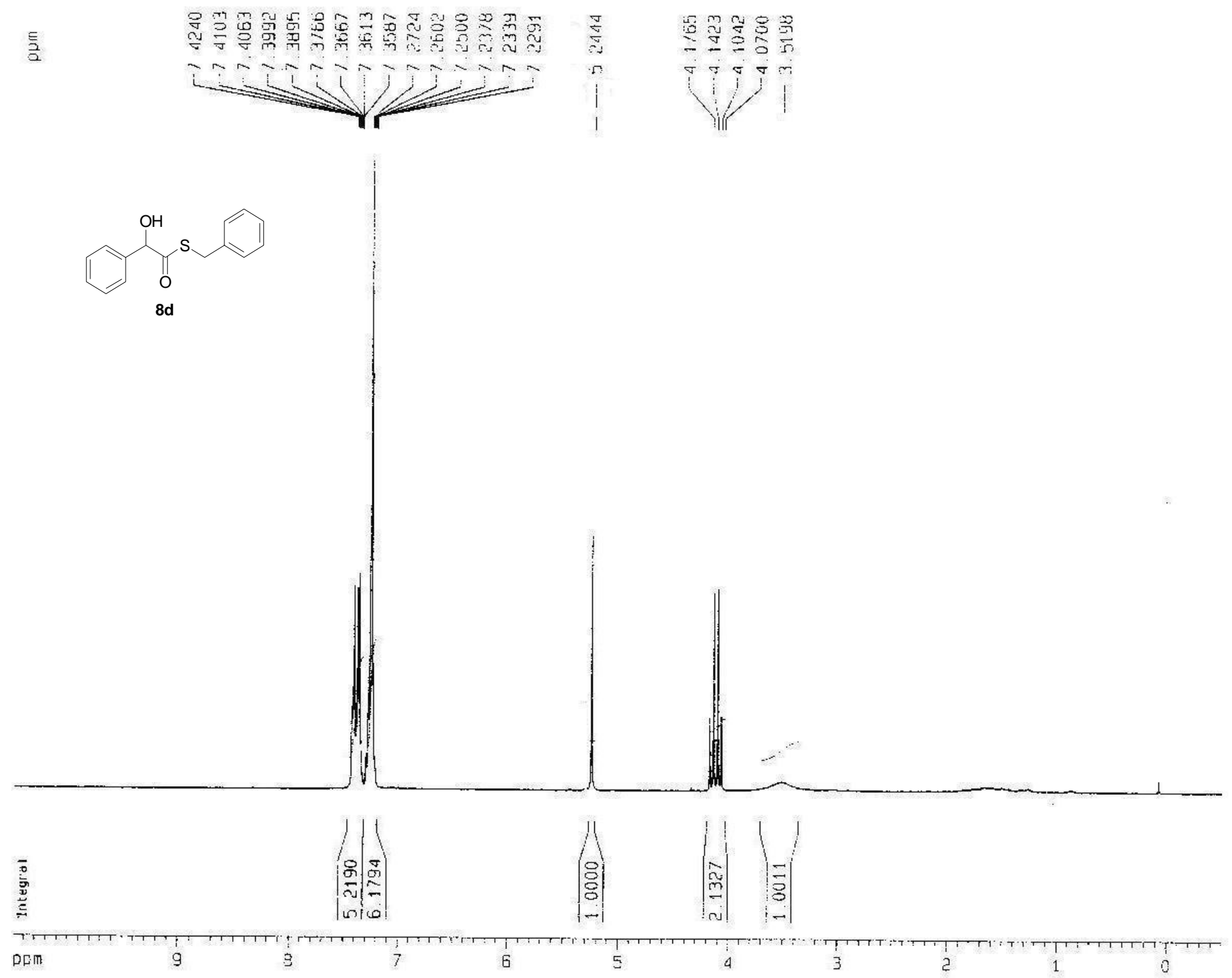

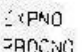

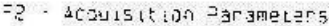

Jate _ $\quad 20060523$

ine $\quad 5.32$

instolus soect

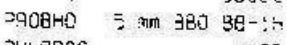

30.7906

SoL

vs

JW $3905.204 \mathrm{~Hz}$

२G $\$ 22 \overline{\mathrm{a}}$

83. 500 usec

6 50 'se

₹0000000 $\mathrm{sec}$

J.

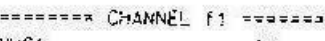

${ }_{21} \quad 10.10 \mathrm{usec}$

$\begin{array}{lrl}31.1 & 3.90 \mathrm{JB} \\ \text { IF01 } & 400 \quad 1326009 \mathrm{MHz}\end{array}$

=2 - рracassung paramerers

II 16384

FF" $400.130009:$ Ma

NOW

2
-3

36

$5 \mathrm{M}$

0

0

1D NMA DiOK parameters

cx $20.00 \mathrm{~cm}$

$\begin{array}{ll}\mathrm{Fr} & 10.50 \mathrm{~cm}\end{array}$

$\begin{array}{lll}-1 P & 10500 \mathrm{pom} \\ -1 & 420137 \mathrm{~Hz}\end{array}$

-2p -12.500 oon

$2 \quad-200.07 \mathrm{~Hz}$

$\begin{array}{lr}\rightarrow \text { DPCM } & 055000 \mathrm{ppm} / \mathrm{cm} \\ \rightarrow Z C H & 220.07152 \mathrm{iz} / \mathrm{cm}\end{array}$ 


ह⿳亠口冋口

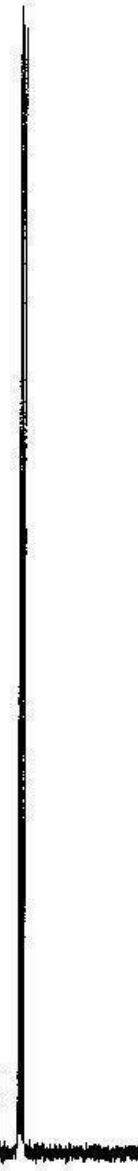

I
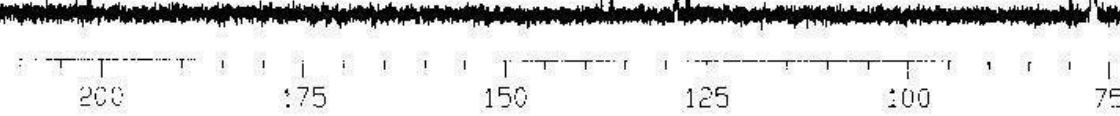

1
75

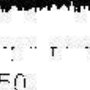

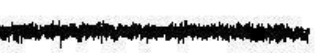
$T-1$
25 
4 soer:- an: :

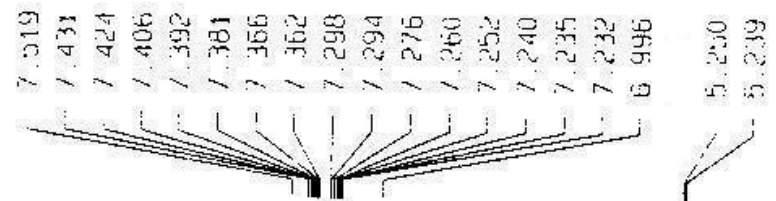

=3 - Arju $25: t$ : on دar эmeters

iate- z0050a15

INSTRUM 15. 78

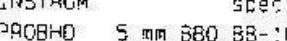

गULPROG I3:

To 12584

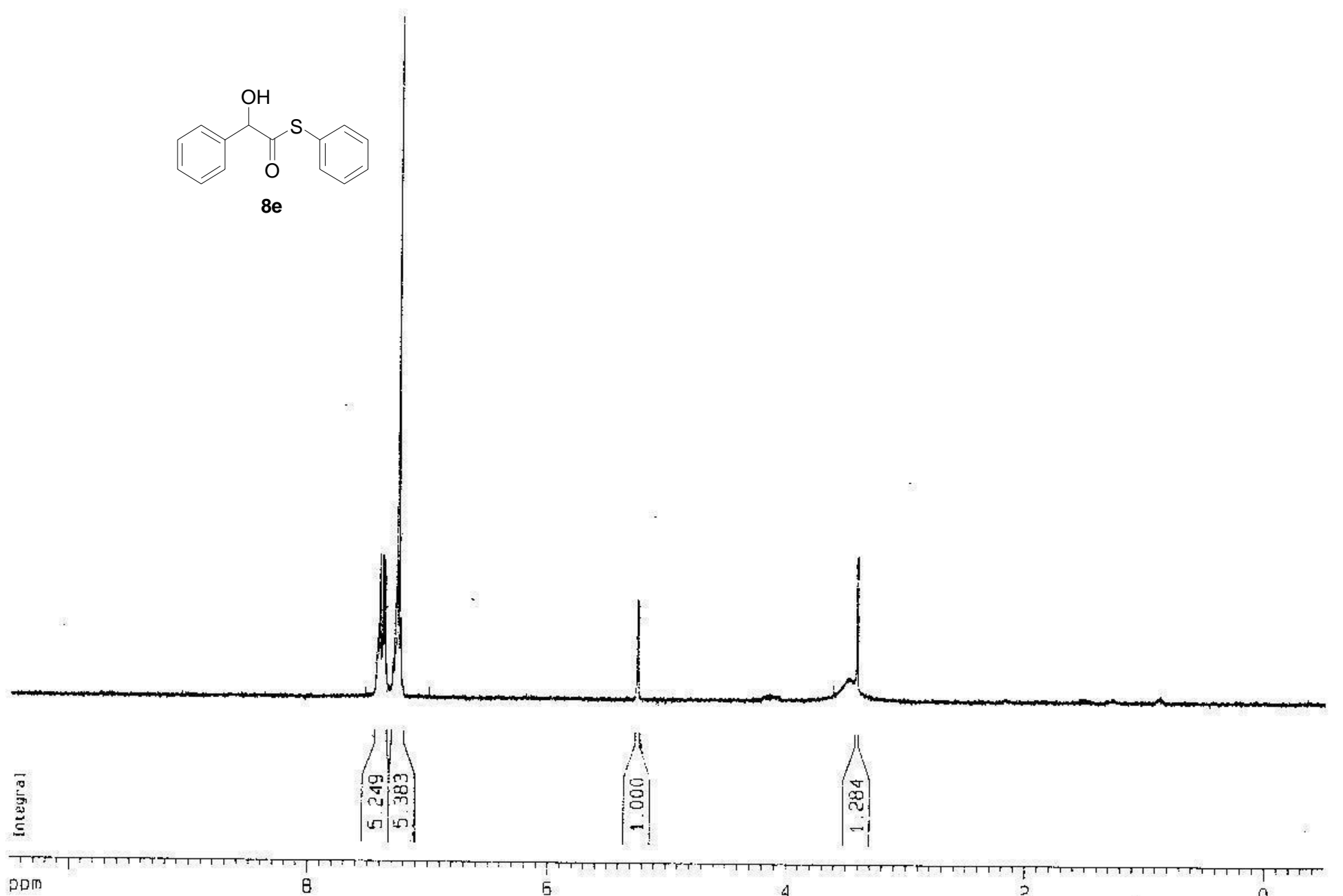

vs

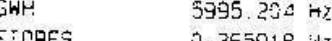

I उË 37565

545.1
33.400
isse

JE 50 use

$1-500000$

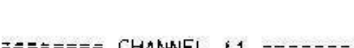

VLiC:

$21 \quad$ to. 10 wsec

3.00
560

Fe - Processing paramecars

51 16384

SF $\quad 400.1300095 \mathrm{MHZ}$

WOW

$5 \mathrm{sB}$
$-\mathrm{B}$
50

$$
\begin{gathered}
300095 \mathrm{MHZ} \\
\equiv: 4 \\
0 \\
0.10 \mathrm{HZ} \\
0
\end{gathered}
$$

to NMR plot oar ameters

$\begin{array}{ll}\text { CX } & 20.00 \mathrm{tm} \\ \mathrm{Cy} & 3000 \mathrm{~m}=\mathrm{m}\end{array}$

F1P $\quad 10.500 \mathrm{ppm}$

$\begin{array}{ll}=1 & 4201.37 \mathrm{~Hz} \\ =20 & -0.500 \mathrm{ogm}\end{array}$ 


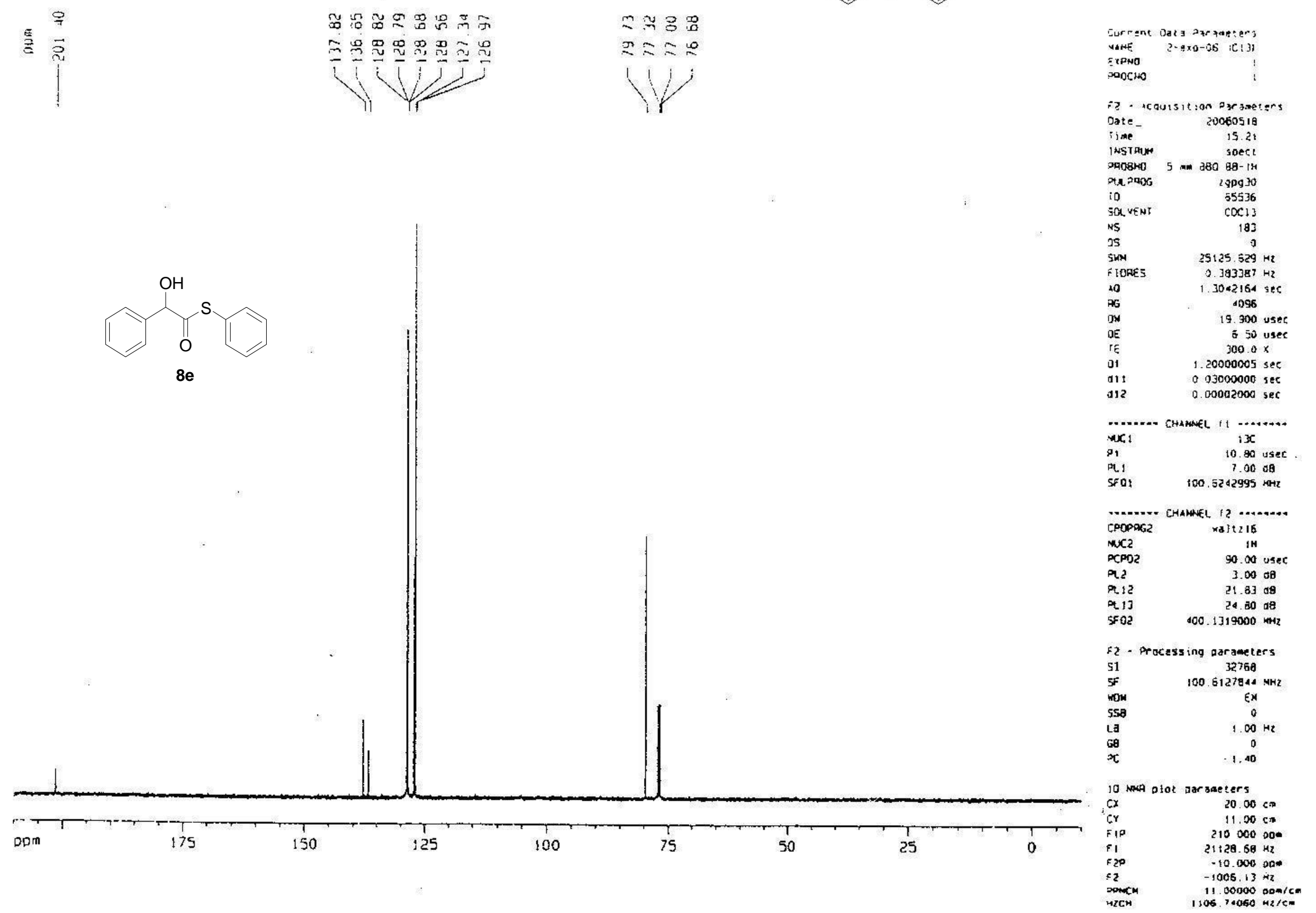




\section{$H$ spectrum of}

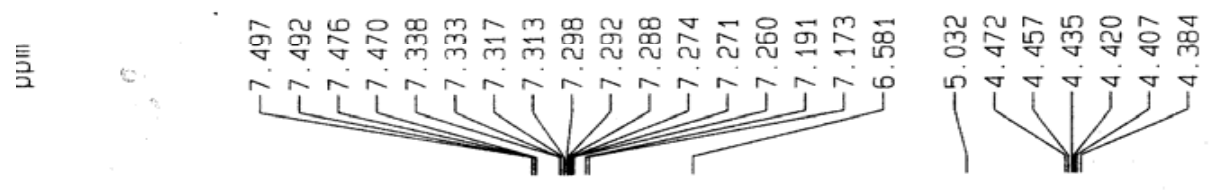

NAME

PROCNO

F2 - Acquisition Parameters

Date _ 20051213

Time

INSTRUM spect PROBHD $5 \mathrm{~mm}$ BBO BB-1H $\begin{array}{lr}\text { PULPROG } & 2930 \\ \text { TO } & 16384\end{array}$

SOLVENT CDC13

NS

DS 0

SWH $\quad 5995.204 \mathrm{H}_{2}$

RG $\quad 322.5$

DW 83.400 usec

6.50 usec

$\begin{array}{lc}\text { TE } & 300.0 \mathrm{~K} \\ \text { D1 } & 1.50000000 \mathrm{sec}\end{array}$

$==z====$ CHANNEL $f 1==z==z=$

$\begin{array}{ll}\text { NUC1 } & 1 \mathrm{H} \\ \mathrm{P}_{1} & 10.10 \mathrm{usec}\end{array}$

$\begin{array}{lr}\text { PL1 } & 3.00 \mathrm{~dB} \\ \text { SFO1 } & 400.1326008 \mathrm{MHz}\end{array}$

SI - Processing parameters

SF $\quad 400.1300099 \mathrm{MHz}$

WOW EM

$\begin{array}{lc}\text { SSB } & 0 \\ \text { LB } & 0.10 \mathrm{~Hz}\end{array}$

GB $\quad 0$

10 NMR plot parameters

10 NMR plot parameters
CX $20.00 \mathrm{~cm}$

$\begin{array}{lr}\text { CX } & 20.00 \mathrm{~cm} \\ \mathrm{CY} & 9.00 \mathrm{~cm}\end{array}$

F1P $\quad 10.500 \mathrm{ppm}$

F1 $\quad 4201.37 \mathrm{~Hz}$

$\begin{array}{ll}F 2 P & -0.500 \mathrm{ppm} \\ F 2 & -200.07 \mathrm{~Hz}\end{array}$

$\begin{array}{ll}F 2 & -0.500 .07 \mathrm{~Hz} \\ \text { PPMCM } & 0.55000 \mathrm{pDm} / \mathrm{c}\end{array}$

$\begin{array}{lr}\text { PPMCM } & 0.55000 \mathrm{ppm} / \mathrm{cm} \\ \text { HZCM } & 220.07152 \mathrm{~Hz} / \mathrm{cm}\end{array}$

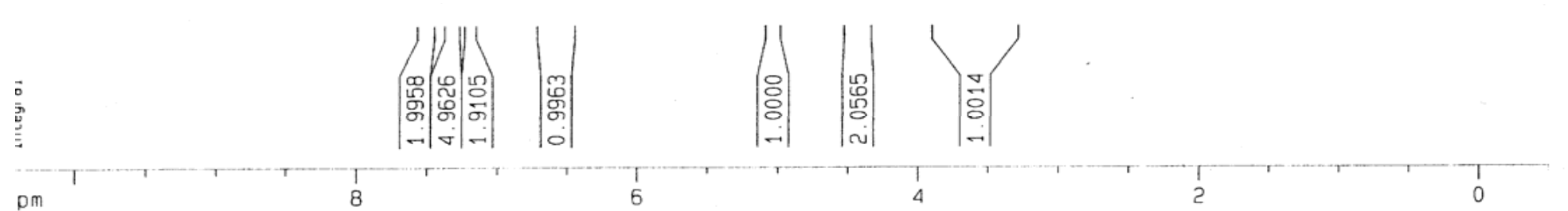


C13 spectrum of

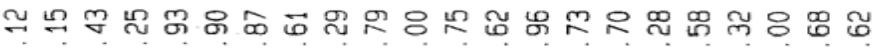

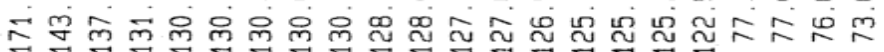
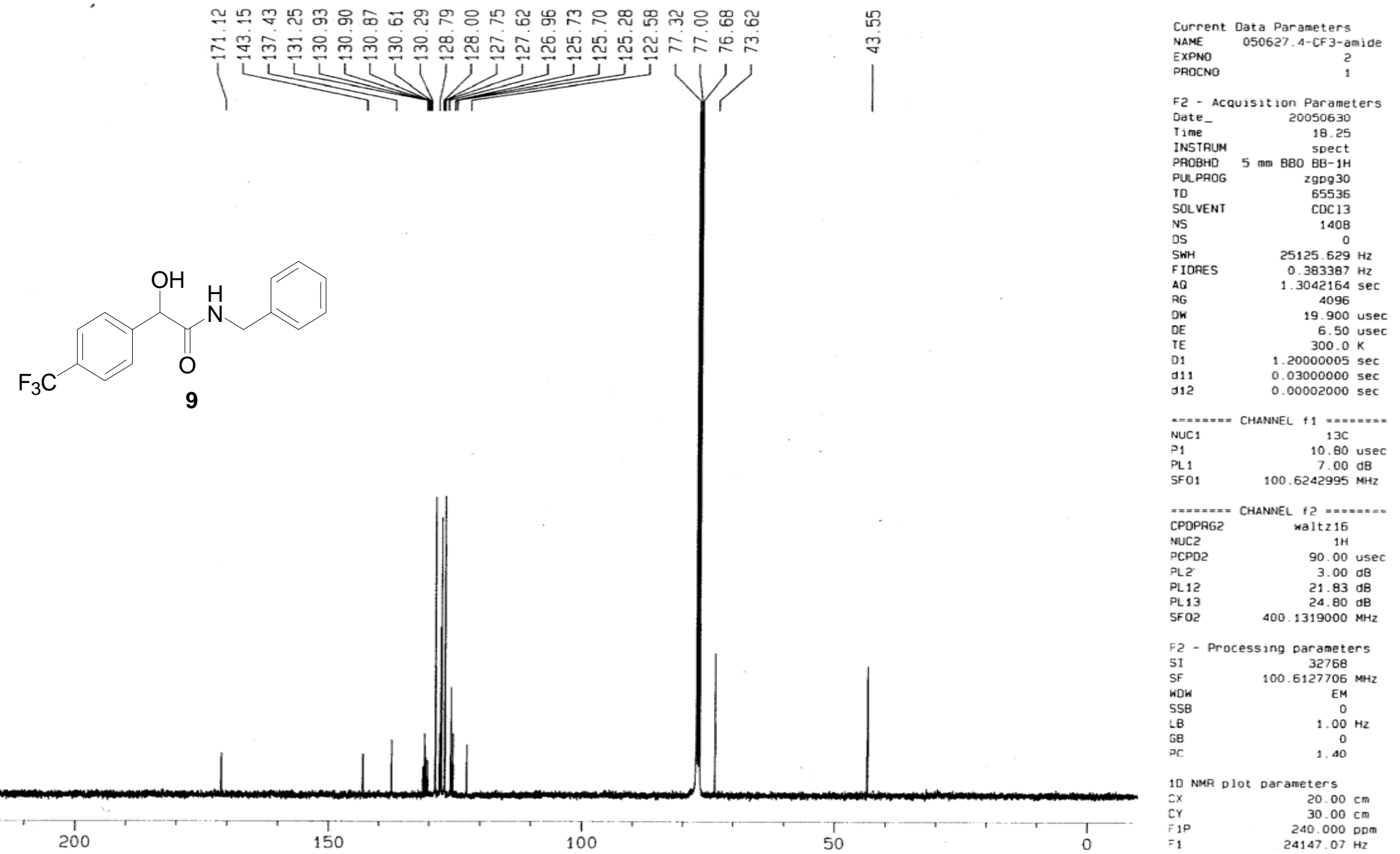

10 NMR plot parameters

10 NMR Dlot Darameters
C. $20.00 \mathrm{~cm}$ 
$H$ spectrum of

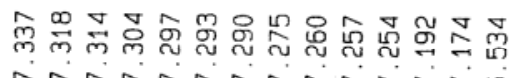

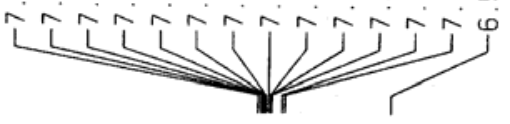

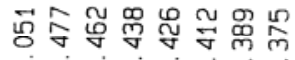

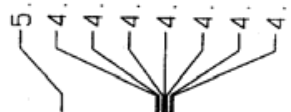

$\mathrm{Cl}$

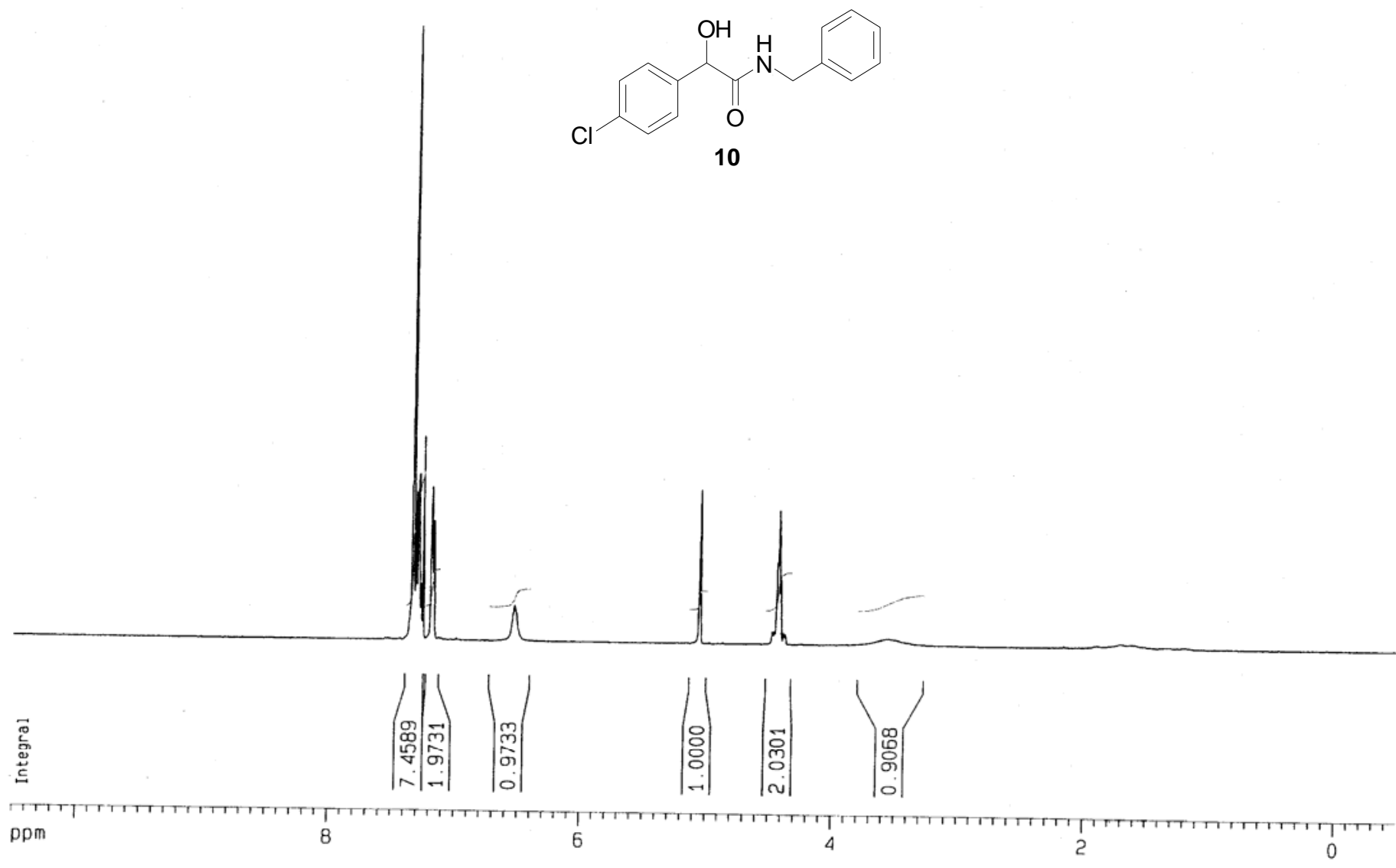

10
Current Data Parameters

NAME 4 CImedelicBnamide

PROCNO 5

F2 - Acquisition Parameter

Date_ 20050319

INSTRUM $\quad 19.50$

PROBHO $5 \mathrm{~mm}$ BBO spect

PULPROG

PULP

SOLVENT

SWH

FIDRES

$A Q$

RG

DE

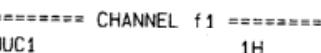

$$
\begin{aligned}
& \begin{array}{ll}
\text { P1 } & 10.10 \text { usec } \\
\text { PL1 } & 3.00 \mathrm{~dB}
\end{array}
\end{aligned}
$$$$
\text { SF01 } 400.1326008 \mathrm{MHz}
$$

F2 - Processing parameters

SI 16384

SF $\quad 400.1300102 \mathrm{MHz}$

WDW

$\begin{array}{ll}\mathrm{LB} & 0 \\ \mathrm{CB} & 0.10 \mathrm{~Hz}\end{array}$

${ }_{P C}^{G B}$

10 NMR plot parameters

CX $\quad 20.00 \mathrm{~cm}$

CY $\quad 9.00 \mathrm{~cm}$

$\begin{array}{lr}\text { Cr } & 10.500 \mathrm{ppm} \\ \text { F1 } & 4201.37 \mathrm{~Hz}\end{array}$

F2P $\quad-0.500 \mathrm{ppm}$

$-200.06 \mathrm{~Hz}$

$\begin{array}{ll}\text { PPMCM } & 0.55000 \mathrm{pDm} / \mathrm{cm}\end{array}$ 

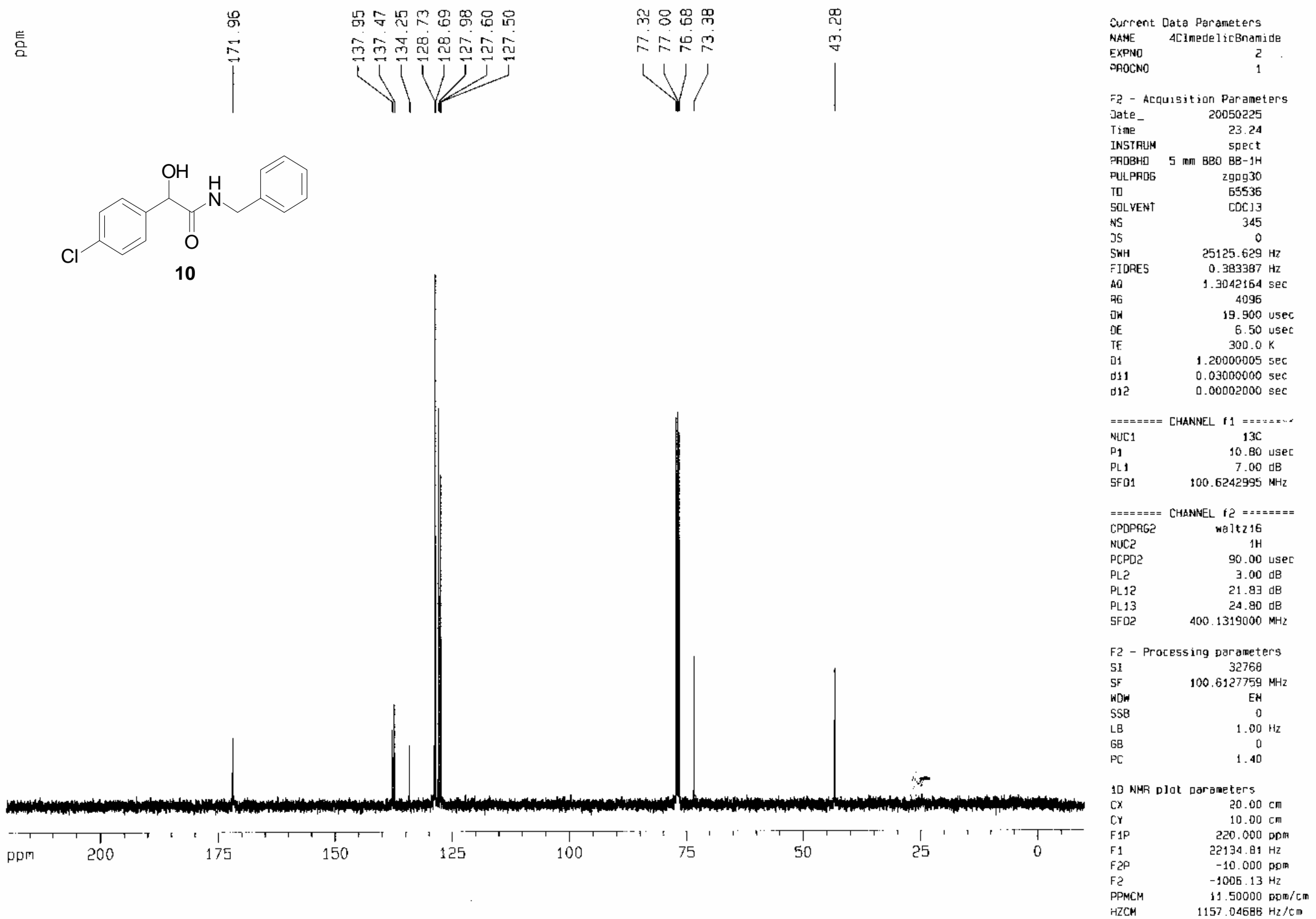
$H$ spectrum of

言

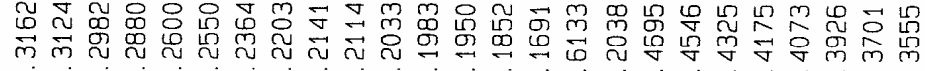

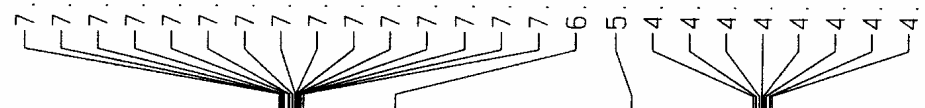

$\mathrm{OH}$

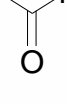

11

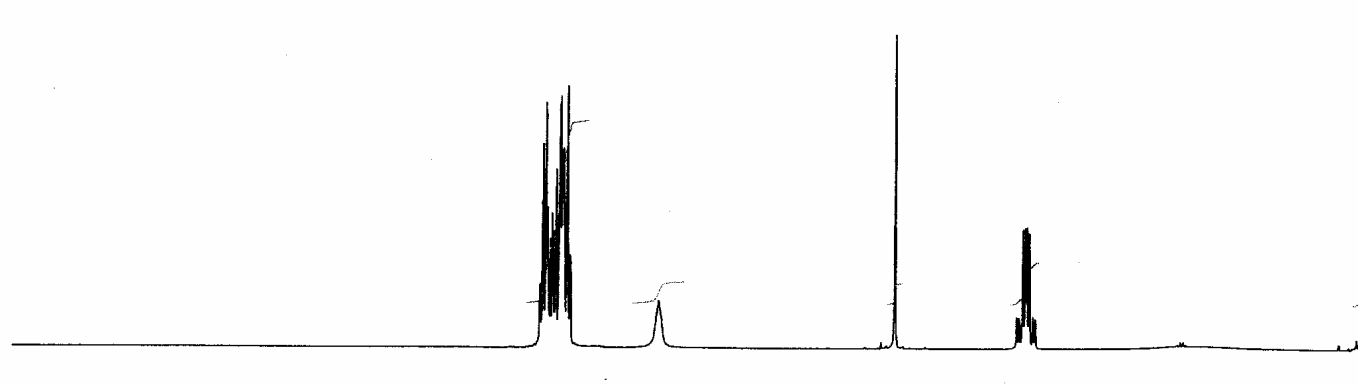

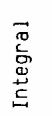

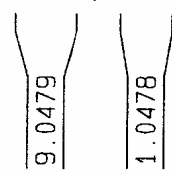

ppm

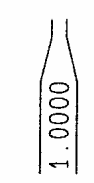

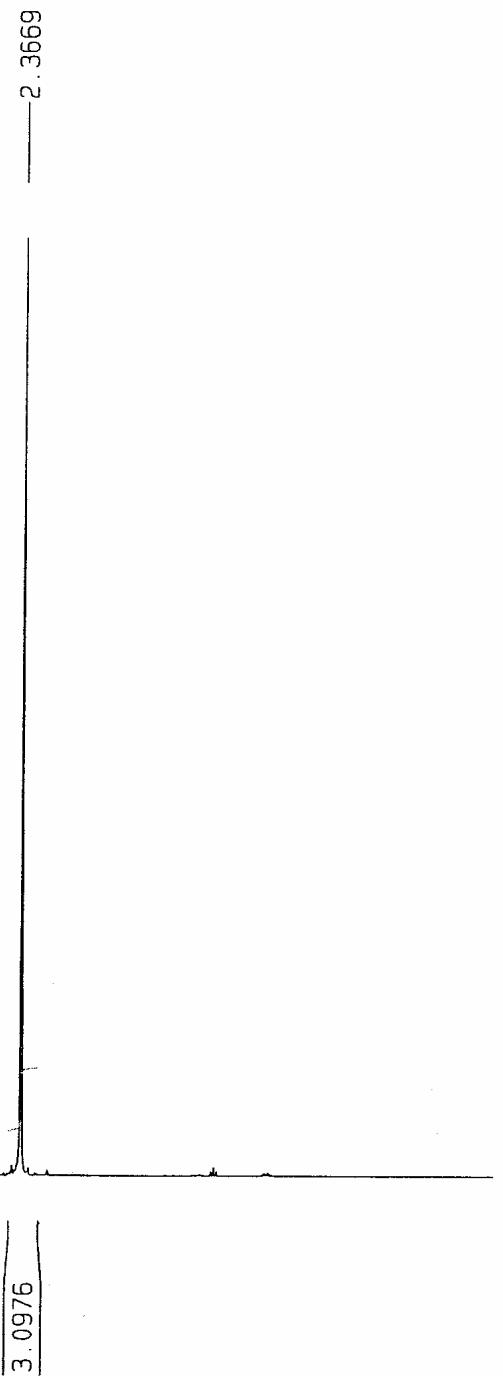

Current Data Parameters

NAME 2-Me-Medaliamide

EXPNO

F2 - Acquisition Parameters

Date _ 20050213

Time 23.40

INSTRUM

PROBMD $5 \mathrm{~mm}$ BBO BB-1H

PULPROG 2930

$\begin{array}{ll}\text { TD } & 16384 \\ \text { SOLVENT } & \text { CDC13 }\end{array}$

NS

$\begin{array}{lr}\text { DS } & 0 \\ \text { SWH } & 5995.204 \mathrm{~Hz}\end{array}$

$0.365918 \mathrm{~Hz}$

AQ $\quad 1.3664756 \mathrm{se}$$$
90.5
$$

83.400 usec

6.50 usec

1. $50000000 \mathrm{sec}$

$====x=x=$ CHANULL $f 1=====5$

$\begin{array}{ll}\text { NUC1 } & 1 \mathrm{H} \\ \text { P1 } & 10.10 \mathrm{useC}\end{array}$

PL1 $3.00 \mathrm{~dB}$

SF01 $400.1326008 \mathrm{MHZ}$

F2 - Processing parameters

SI 16384

SF $\quad 400.1300088 \mathrm{MHZ}$

WDW

SSB
LB

$G B$

$300088 \mathrm{MHZ}$
$\mathrm{EM}$
0
$0.10 \mathrm{~Hz}$

$0.10 \mathrm{~Hz}$

1.00

10 NMR plot parameters

$\begin{array}{ll}\text { CX } & 20.00 \mathrm{~cm} \\ \mathrm{CY} & 10.50 \mathrm{~cm}\end{array}$

$\begin{array}{lrl}\text { CY } & 10.50 \mathrm{~cm} \\ \text { F1P } & 10.500 \mathrm{cDm}\end{array}$

$\begin{array}{ll}\mathrm{CH} & 10.500 \mathrm{Dpm}\end{array}$

$\begin{array}{ll}\text { F1 } & 4201.37 \mathrm{~Hz} \\ \text { FEP } & -0.500\end{array}$

F2 $\quad-200.06 \mathrm{~Hz}$

$\begin{array}{ll}\text { PPMCM } & 0.55000 \mathrm{ppm} / \mathrm{cm}\end{array}$ 
C13 spectrumbufogge: spectrum of

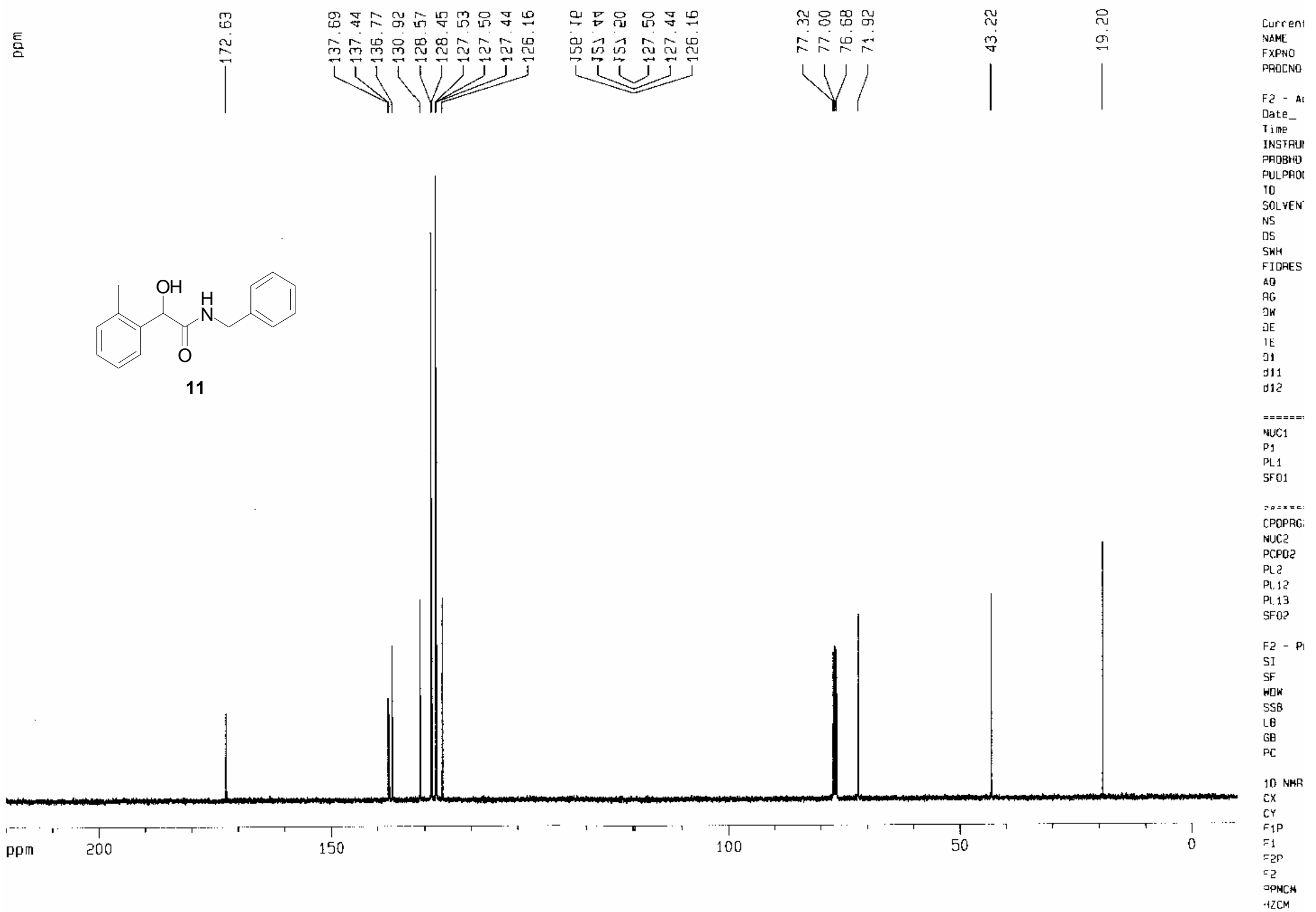


$H$ spectrum of
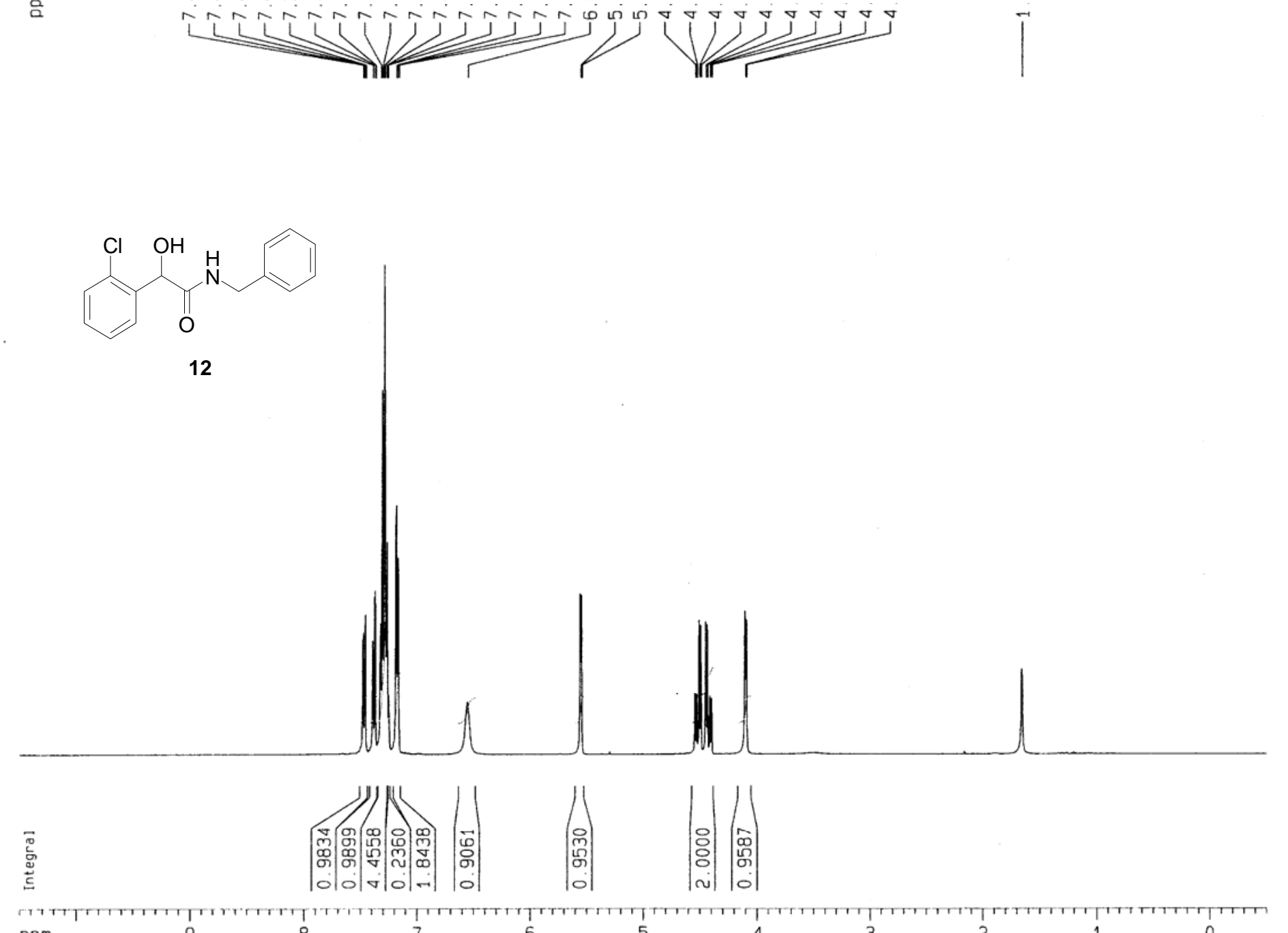

ppm

9

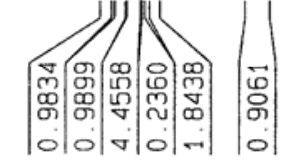

$\mid \begin{aligned} & 1 \\ & 0 \\ & 0 \\ & 0 \\ & 0 \\ & 0\end{aligned}$

$\left|\begin{array}{c|c} & \\ 0 \\ 0 \\ 0 \\ 0\end{array}\right|\left|\begin{array}{c}n \\ 0 \\ 0 \\ 0 \\ 0\end{array}\right|$

7

6

5

\begin{abstract}
4
\end{abstract}
2

Current Data Parameters NAME $050520.2-C 1$-amide EXPNO

F2 - Acquisition Parameters

Date 20050520 15.07 INSTRUM spect PROBHD $5 \mathrm{~mm} \mathrm{BBO} \mathrm{BB-1 \textrm {H }}$ $\begin{array}{lr}\text { PULPROG } & 2930 \\ \text { TD } & 16384\end{array}$ SOLVENT $\quad$ COC13 NS

SWH $\quad 5995$ FIDRES $\quad 0.365918 \mathrm{~Hz}$ AQ $\quad 1.3664756 \mathrm{sec}$ RG 1613 DW
DE $\quad 83.400$ usec TE $01 \quad 1.50000000 \mathrm{sec}$ $=======$ CHANNEL
NUC1 $1==z====$
$1 \mathrm{H}$ $P_{1} \quad 10.10$ usec SF01 400.1325008

2 - Processing parameters SI 16384 SF $\quad 400.1300099$ MHZ WDW $\quad$ EM GB 1.00 10 NMR plot parameters CX $\quad 20.00 \mathrm{~cm}$ $10.500 \mathrm{~cm}$ F1 $\quad 10.500 \mathrm{ppm}$ F2P $\quad-0.500 \mathrm{pDm}$ F2 $\quad-200.07 \mathrm{~Hz}$ $\begin{array}{lr}\text { PPMCM } & 0.55000 \mathrm{ppm} / \mathrm{cm} \\ \mathrm{HZCM} & 220.07152 \mathrm{HZ} / \mathrm{cm}\end{array}$ 
C13 spectrum of

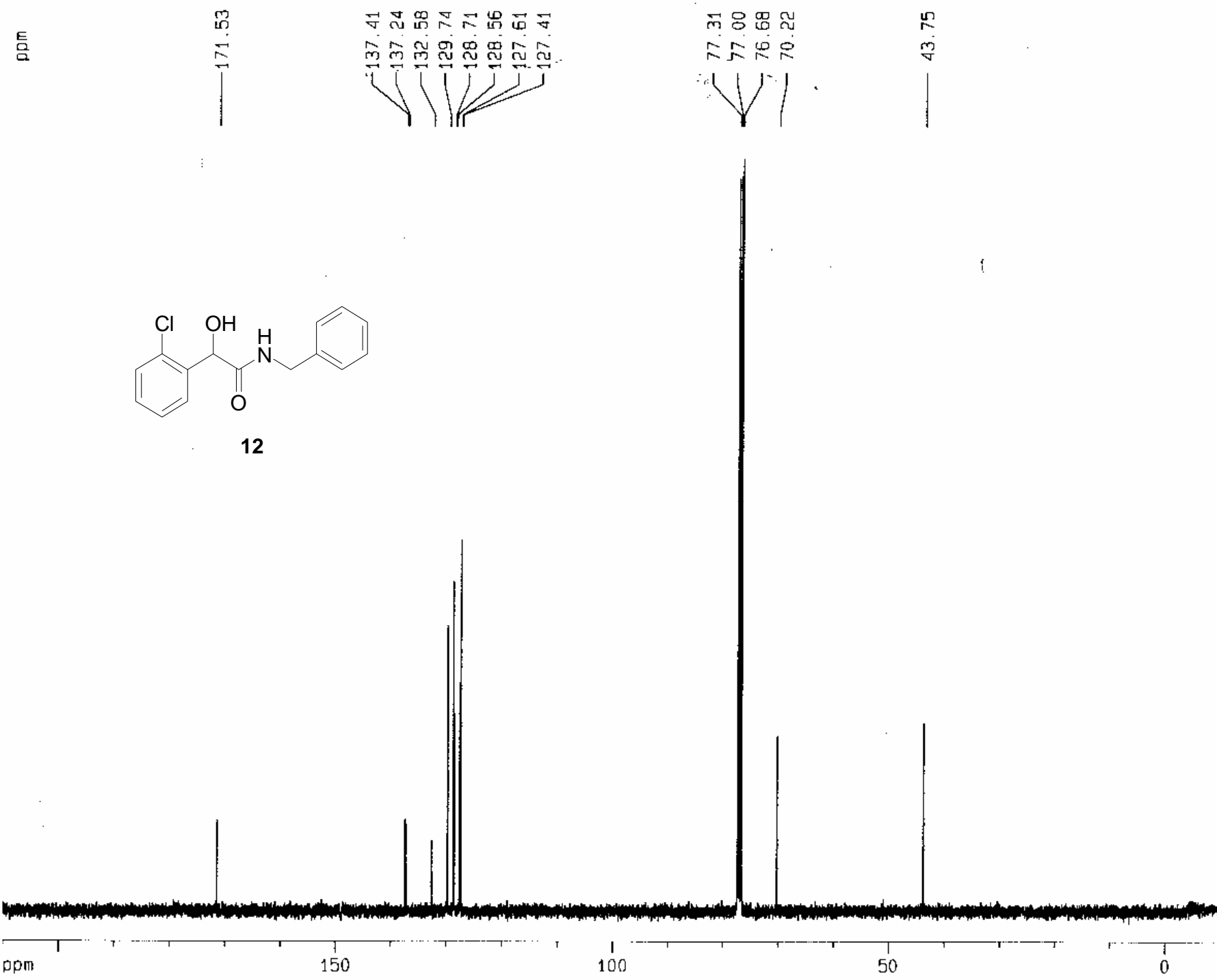

$$
\begin{aligned}
& \text { Current Data Parameters }
\end{aligned}
$$

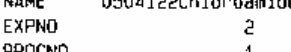

$$
\begin{aligned}
& \text { F2 - Acquisition Parameters } \\
& \text { Date_ } 20050412 \\
& \begin{array}{lr}
\text { I ImE } & 18.03 \\
\text { INSIRUM } & \text { 5pect } \\
\text { PFOBHD } & 5 \mathrm{~mm} \text { BBo BB-1H }
\end{array} \\
& \text { PULPFOG } 5 \text { an }
\end{aligned}
$$

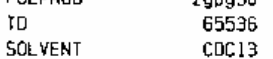

$$
\begin{aligned}
& \begin{array}{lr}
\mathrm{NS} & 220 \\
05 & 0
\end{array} \\
& \begin{array}{l}
05 \\
5 \mathrm{HH}
\end{array} \\
& \begin{array}{ll}
0.399397 \mathrm{~Hz} \\
\mathrm{AB} & 1.3042154 \mathrm{5EC}
\end{array}
\end{aligned}
$$

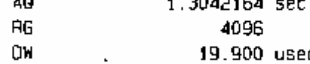

$$
\begin{aligned}
& \begin{array}{r}
\text { 19. } 900 \text { usec } \\
\text { 6. } 50 \text { use }
\end{array} \\
& 300.0 \mathrm{~K} \\
& \text { 1. } 0.00000005 \mathrm{sec} \\
& 0.000020000 \text { sec }
\end{aligned}
$$

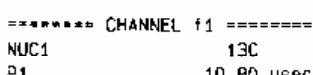

$$
\begin{aligned}
& \text { LL, 10. Bo use } \\
& 100.6242995 \mathrm{MHZ}
\end{aligned}
$$

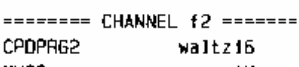

$$
\begin{aligned}
& \text { NuC2 } \quad 1 \text { PCPR }
\end{aligned}
$$

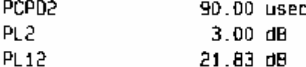

$$
\begin{aligned}
& \begin{array}{ll}
24.80 \mathrm{dg} \\
\mathrm{L} 13
\end{array} \\
& \text { SFO2 400. } 1319000 \mathrm{MHZ} \\
& \text { F́2 - Processing parameters } \\
& \begin{array}{lc}
\text { SI } & 32768 \\
\text { SF } & 100.6127744 \\
\mathrm{MHz}
\end{array} \\
& \text { Naw } \\
& \begin{array}{lc}
\text { SSE } & 0 \\
\mathrm{LB} & 1.00 \mathrm{~Hz} \\
\mathrm{~GB} & 0 \\
\mathrm{PC} & 1.00
\end{array} \\
& \text { 10 NMA Dlot Daraneters }
\end{aligned}
$$

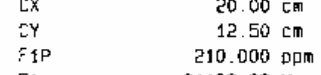


$H$ spectrum of

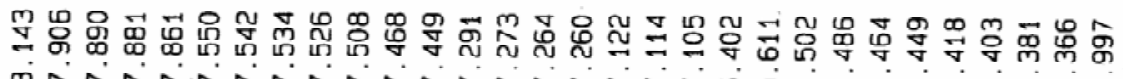

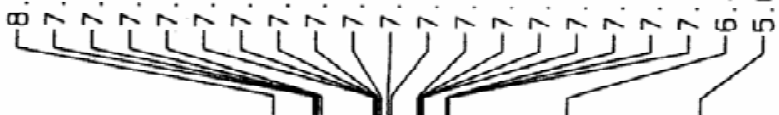

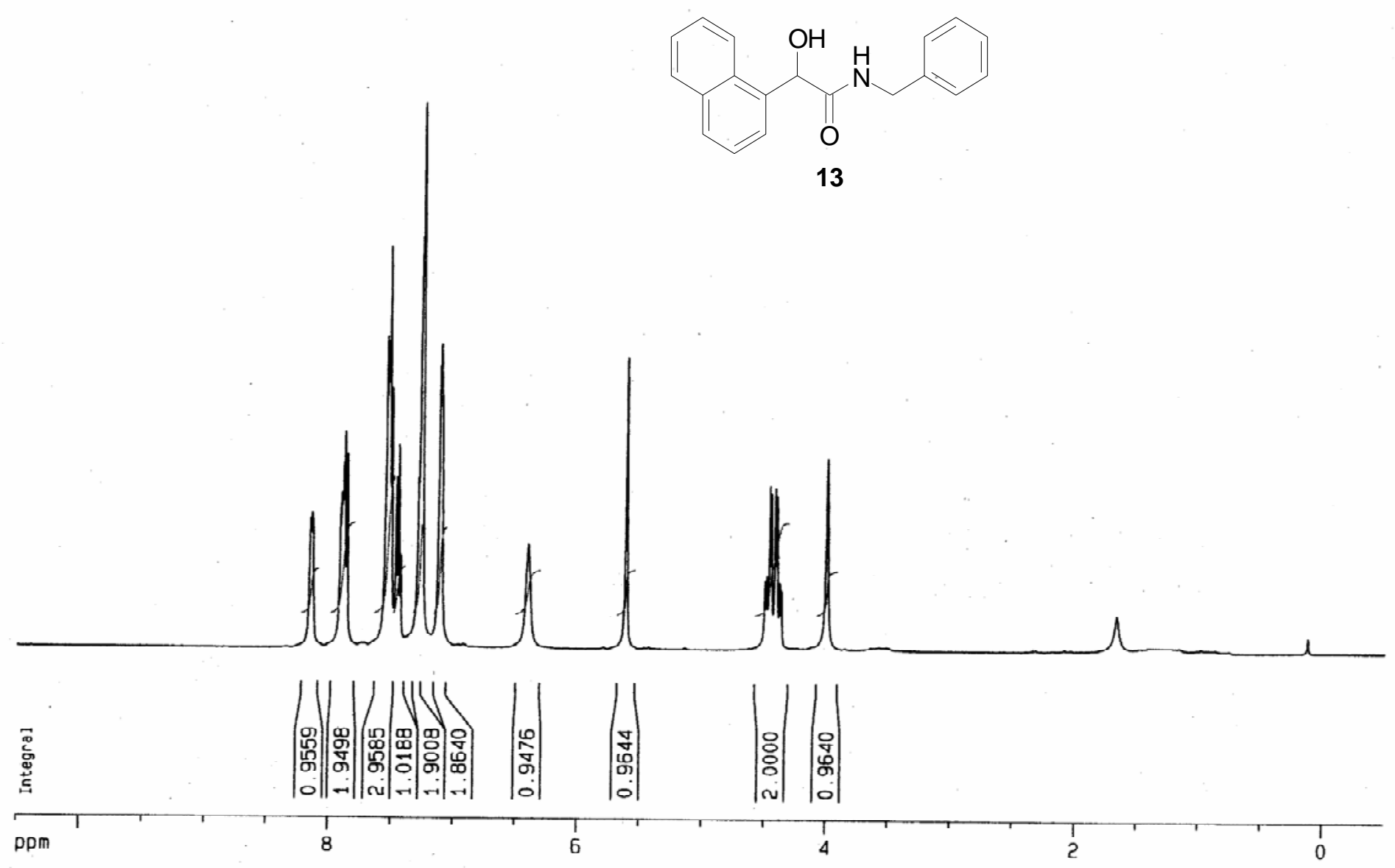

Current Data Parameters AME 050413 inaphamide EXPNO $\quad$ 050413.1naphama EXPNO 1

F2 - Acquisition Parameters Date_ 20050414 Ime 17.47 PROBHD $5 \mathrm{~mm}$ BBO BB-1H PULPROG 2930 TD 16384 SOLVENT COC13 SWH $\quad 5995.204$ FIDRES $\quad 0.365918 \mathrm{~Hz}$ AQ $\quad 1.3664756 \mathrm{sec}$ $\begin{array}{ll}\text { RG } & 143.7 \\ \text { DW } & 83.400 \text { usec }\end{array}$ DE $\quad 6.50$ usec D1 1.50000000 $=m z=m=$ CHANNEL $f 1=m=z=z$ NUC1 $1 \mathrm{H}$ P1 $\quad 10.10$ usec $\begin{array}{lr}\mathrm{PL} 1 & 3.00 \mathrm{~dB} \\ \mathrm{SF01} & 400.1326008 \mathrm{MHz}\end{array}$

F2 - Processing parameters SI 16384 SF $\quad 400.1299967 \mathrm{MHZ}$ NOW LB. $\quad 0.10 \mathrm{~Hz}$

PC

10 NHR plot parameters cX $20.00 \mathrm{~cm}$ $\begin{array}{lr}\text { Cr } & 8.00 \mathrm{~cm} \\ \text { F1P } & 10.500 \mathrm{DDm}\end{array}$ F1 $\quad 4201.37 \mathrm{~Hz}$

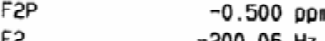
PРмСM $\quad-200.06 \mathrm{~Hz}$ HZCM $\quad 0.55000 \mathrm{ppm} / \mathrm{cm}$ 
C13 spectrum of

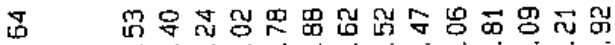

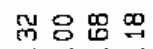

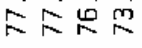

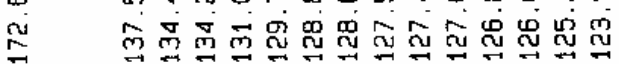

(V)

渵

L L $101 \%$

\%

r

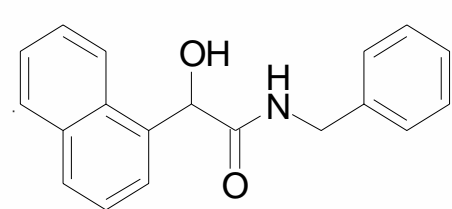

13

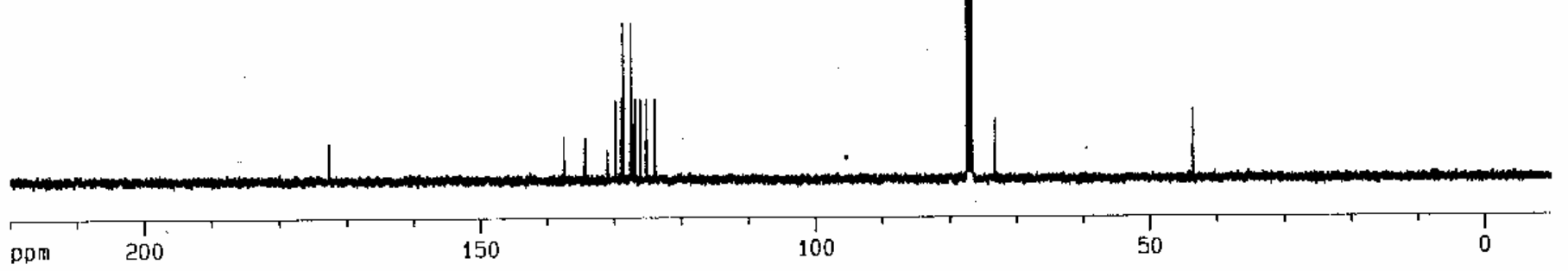

ppm

150
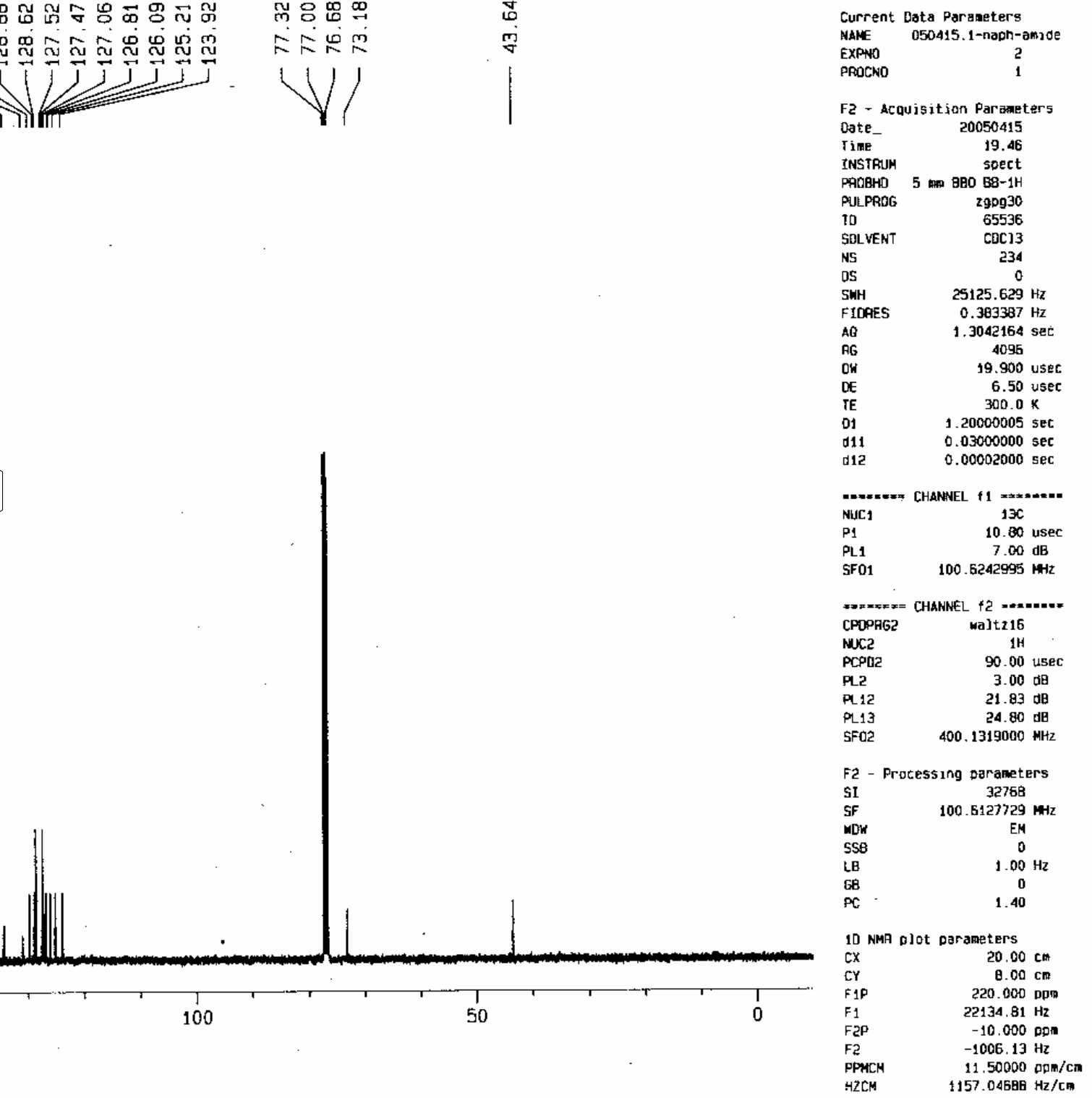
$H$ spectrum of

言

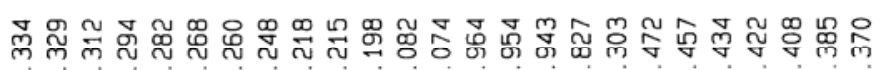

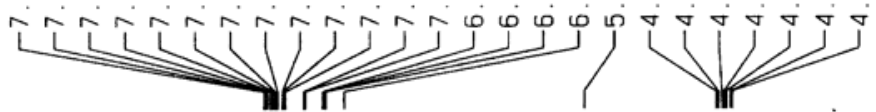

橸品

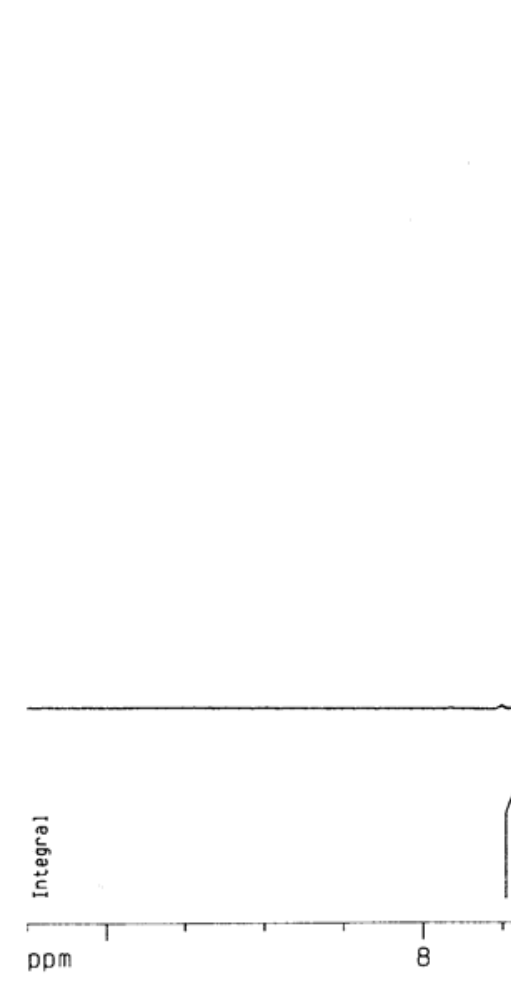

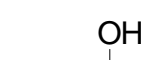
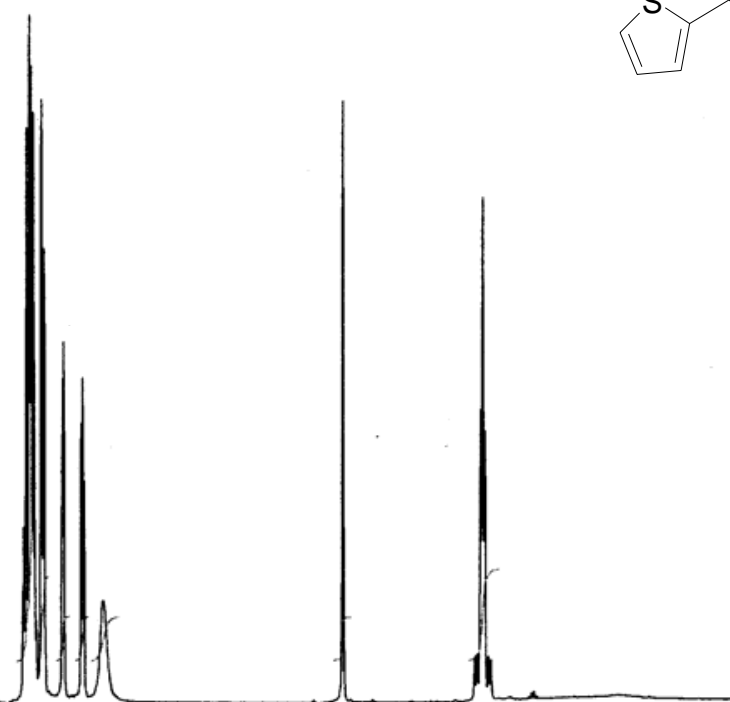

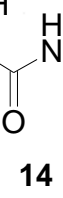

Current Data Parameters

NAME BnNthiophen

PROCNO

Parameters

Date_

Time $\quad 14.34$

Spect
PROBHO $5 \mathrm{~mm} B B O \mathrm{BB}-1 \mathrm{H}$ PULPROG $\quad 2930$ TD 16384

$\begin{array}{ll}\text { TD VOLNT } & 16384 \\ \text { COC13 } & \end{array}$ NS

SWH

FIDRES $\quad 5995.204 \mathrm{~Hz}$

1. $3659756 \mathrm{~Hz}$

1.363 .7

DW $\quad 83.400$ usec

6.50 usec

D1 $\quad 1.50000000 \mathrm{sec}$

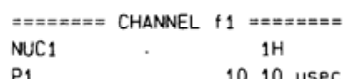

P1 $\quad 10.10$ usec

SF01 400.1326008 MHZ

F2 - Processing parameters

SI 16384

SF $\quad 400.1300088 \mathrm{MHz}$

$\begin{array}{lc}\text { WOW } & \text { EM } \\ \text { SSB } & 0\end{array}$

$\begin{array}{lc}\text { LB } & 0.10 \mathrm{~Hz} \\ \text { GB } & 0\end{array}$

10 NMR plot parameters

CX $\quad 20.00 \mathrm{~cm}$

$\begin{array}{lr}\mathrm{Cr} & 8.00 \mathrm{~cm} \\ \mathrm{Fip} & 10.500 \mathrm{~cm}\end{array}$

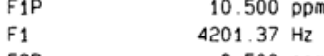

F2P $\quad-0.500 \mathrm{ppm}$

PPMCM $\quad-200.06 \mathrm{~Hz}$

$\begin{array}{ll} & 0.55000 \mathrm{pDm} / \mathrm{cm} \\ \mathrm{HZCM} & 220.07150 \mathrm{~Hz} / \mathrm{cm}\end{array}$ 
C13 spectrum of
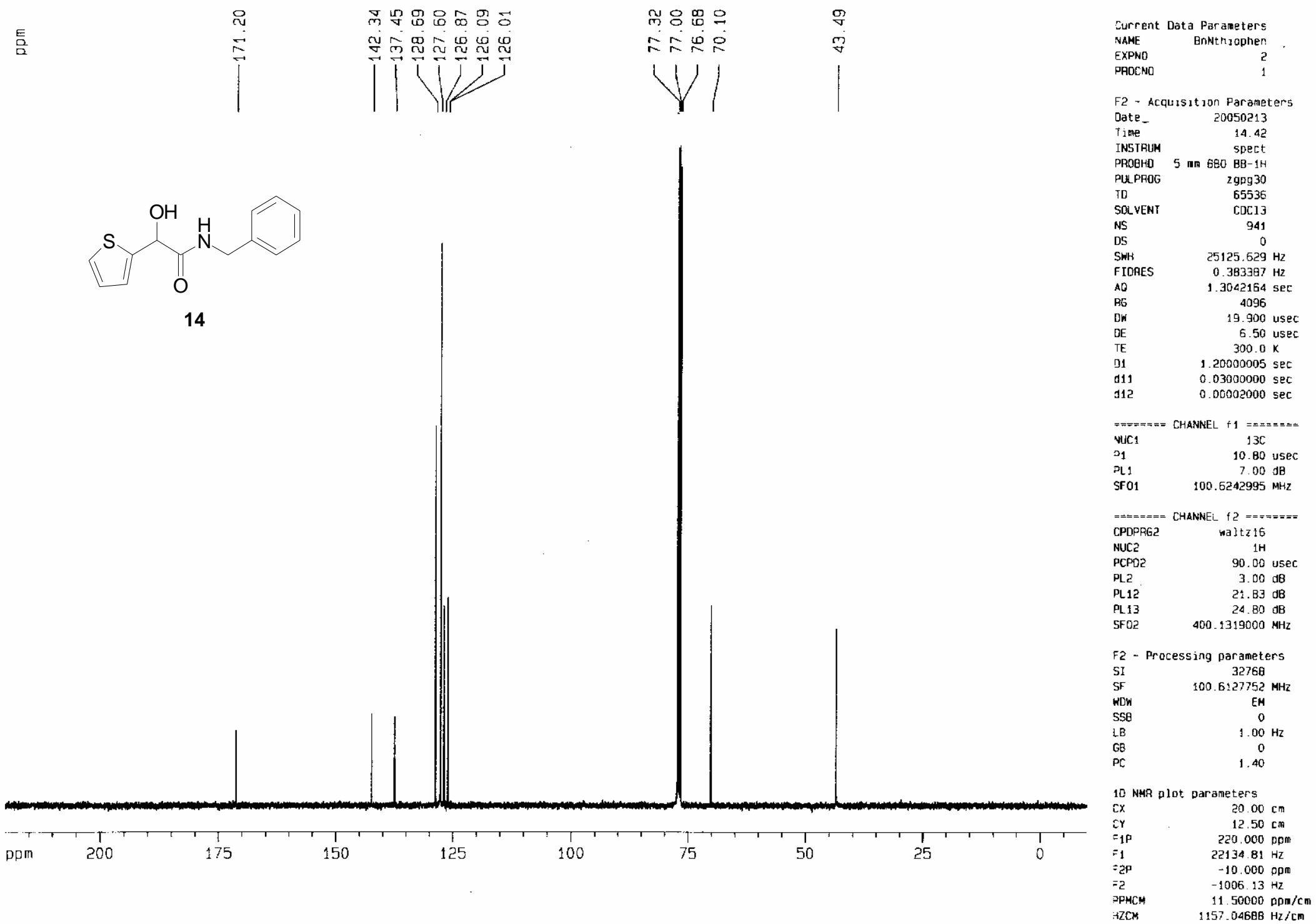
H spectrum of

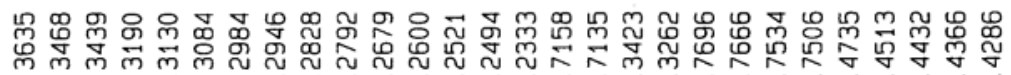
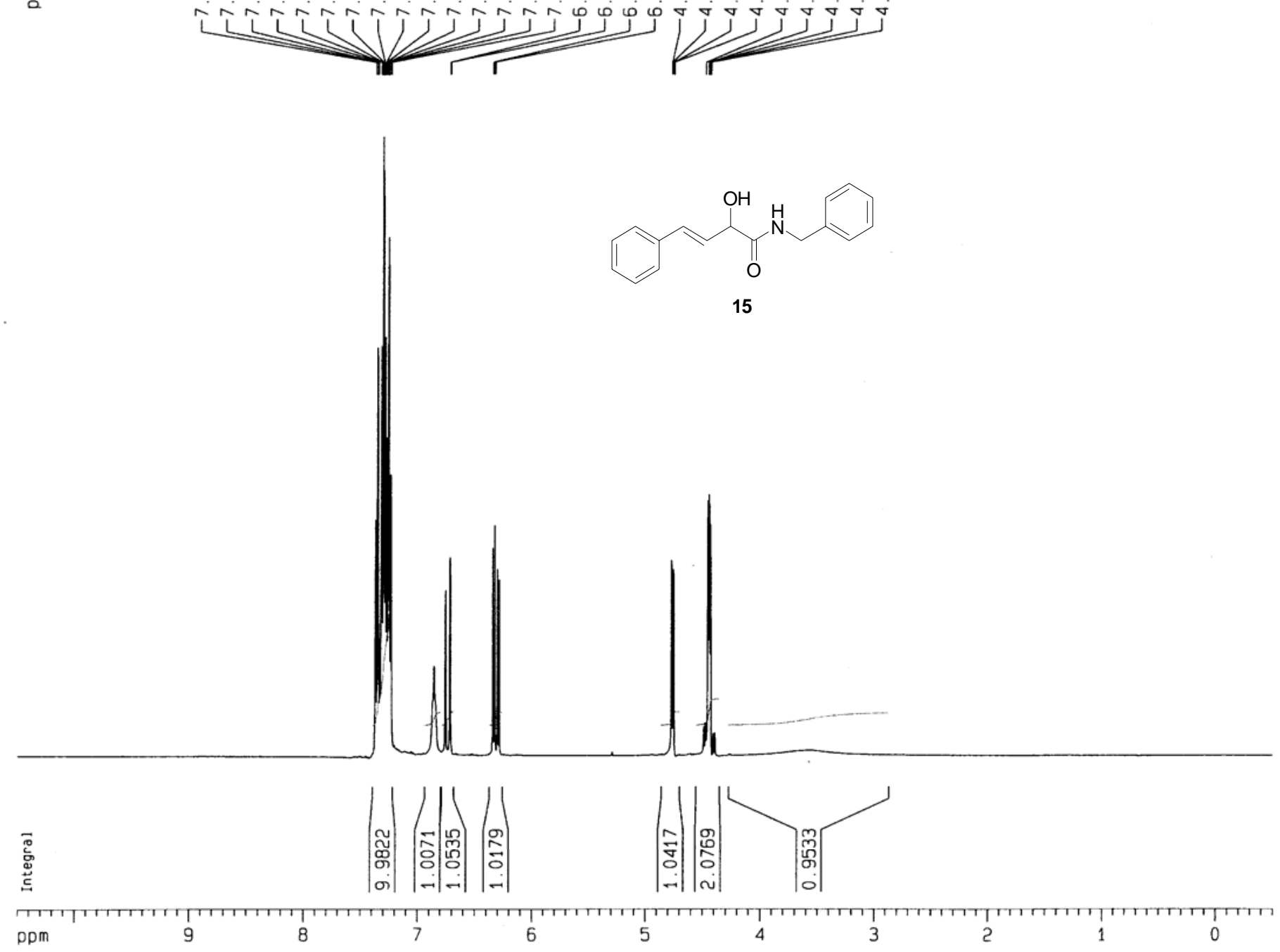

Current Data Parameters

NAME cinamylmedelate

EXPNO

Procio

- Acquisit Parameters

Date_ 20050404

Time

12.06

PROBHD $5 \mathrm{~mm} \mathrm{BBO} \mathrm{spect}$

PULPROG 2930

Th

2930
6384

SOLVEN

NS

SWH

-16
-16
59.204

FIDRES $\quad 5995.204 \mathrm{~Hz}$

$0.365918 \mathrm{~Hz}$

$1.3664756 \mathrm{sec}$
128

OW 83.400 usec

DE 6.50 use

$01 \quad 1.50000000 \mathrm{sec}$

$== \pm=z=z$ CHANNEL $f 1= \pm= \pm= \pm$

P1 $1 \mathrm{H}$

$\begin{array}{lr}\text { PL1 } & 3.00 \mathrm{~dB} \\ \text { SF01 } & 400.1326008 \mathrm{MHz}\end{array}$

F2 - Processing parameters

SI 16384

SF $\quad 400.1300091$ MHz

WOW

$\begin{array}{lr}\text { SSB } & 0 \\ L B & 0.10 \mathrm{~Hz}\end{array}$

$\begin{array}{lr}\text { GB } & 0 \\ \text { PC } & 1.00\end{array}$

10 NMR plot parameters

10 NMR plot parameters

$\begin{array}{ll}\text { CX } & 20.00 \mathrm{~cm} \\ \mathrm{CY} & 10.00 \mathrm{~cm}\end{array}$

F1P $\quad 10.500 \mathrm{ppm}$

F1 $\quad 4201.37 \mathrm{~Hz}$

$-0.500 \mathrm{PDD}$
$\mathrm{F} 2 \mathrm{P}$

PPMCM $\quad 0.55000 \mathrm{ppm} / \mathrm{cm}$ 

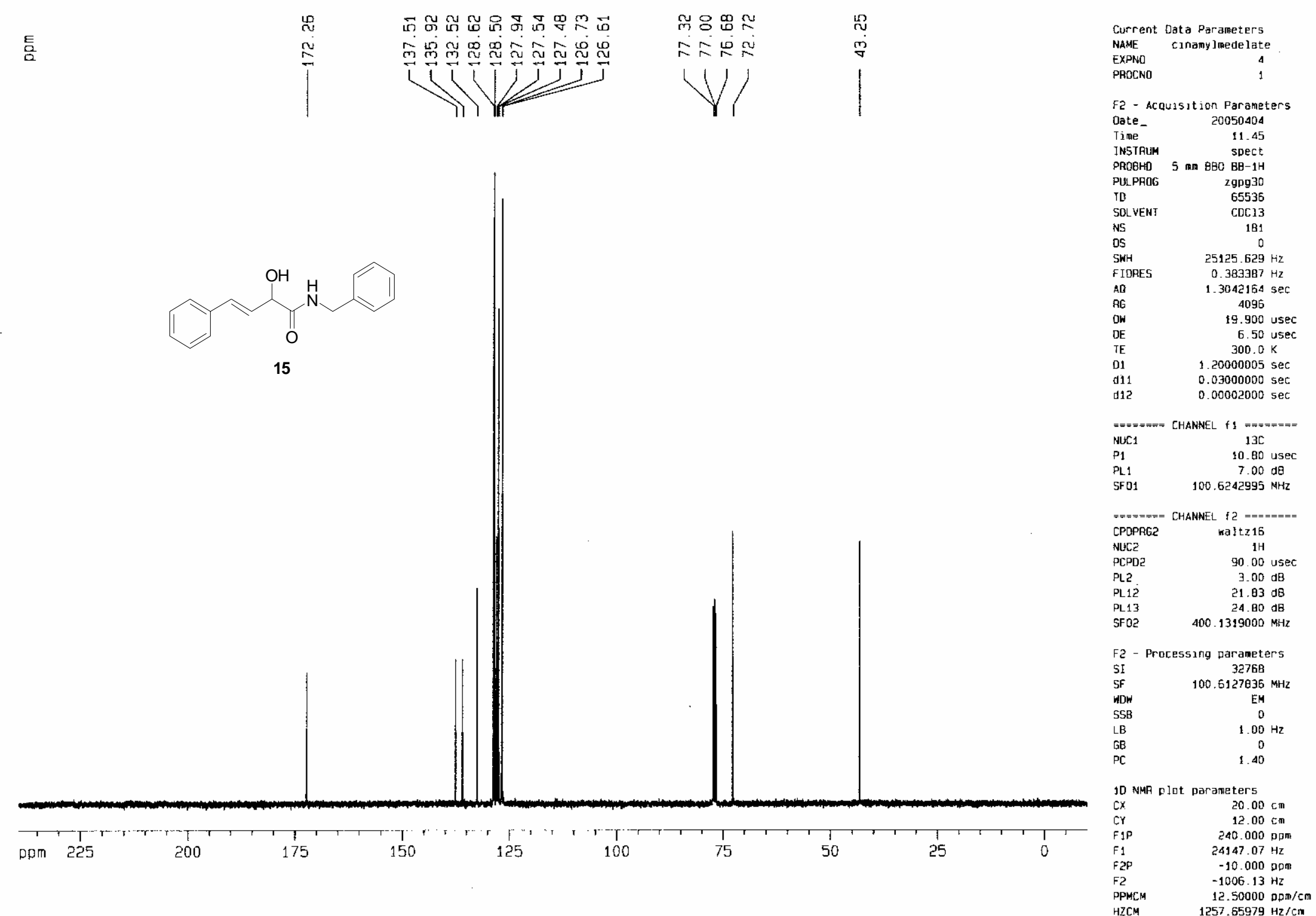
H spectrum: of

틍

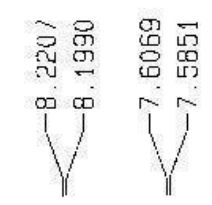

$\mathrm{OH}$

$\mathrm{O}_{2} \mathrm{~N}$ $\int^{\mathrm{s}}$

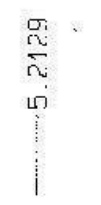

5an--HT-4л了tro

PROCNO

1

-2 - MrQu15] inn Parameters

Date_ 20060109

Time 12.05

ThSTRUM SPEC:

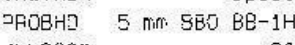

"ULPROG

SCLVEN

is

SW

SWH

40

$\rightarrow G_{3}$

$3 \mathrm{~W}$

TE

16
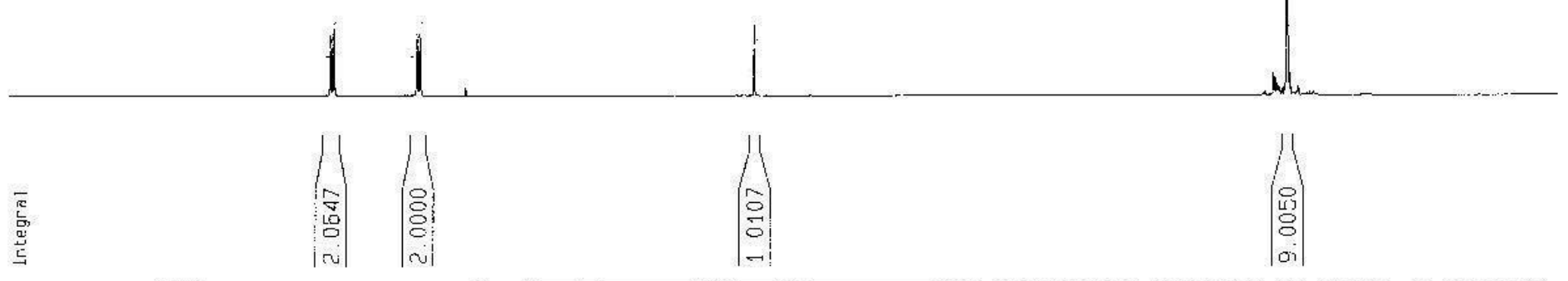

16384
$\operatorname{CDC} 13$
1

$5995.204 \cdot 12$

0.365918 th

1. 3664756 s.e:

71.8

83. $400 \mathrm{usec}$

6. 50 บ5อ $300.0 \mathrm{~K}$ 1. $50000000 \mathrm{sec}$

$======$ CHANNEL $f 14=====$

vert $1 \mathrm{H}$

$\begin{array}{ll}\text { PL } & 10.10 \text { U5e } \\ \text { PL1 } & 3.00 \mathrm{gB}\end{array}$

PL1 $\quad 400.132600 \mathrm{MHHz}$

$\therefore$ - Processing parameters

SI - Processing parameters

$\begin{array}{lr}\text { SI } & 16384 \\ \text { it } & 400.1300095 \mathrm{MHz}\end{array}$

NIOW $\quad 400.1300095$

$\begin{array}{lr}\text { NOW } & \text { EM } \\ S S 3 & 0\end{array}$

$3.10 \mathrm{~Hz}$

$\triangle B$

10. NMA plot paramerers

ax $20.30 \mathrm{~cm}$

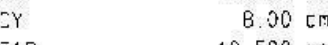

$=1 \quad 10.520 \mathrm{ppm}$

$\begin{array}{ll}-1 & 420 \div .37 \mathrm{~Hz} \\ -2 \mathrm{P} & -0.500 \mathrm{pFnz}\end{array}$

$=5 \quad-200.0 \% \mathrm{~Hz}$

$\quad 0.55050 \mathrm{cDm} / \mathrm{Cm}$

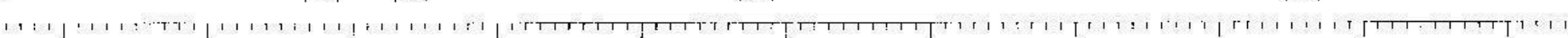

$0 \mathrm{non}$

5

5

3

?

1 

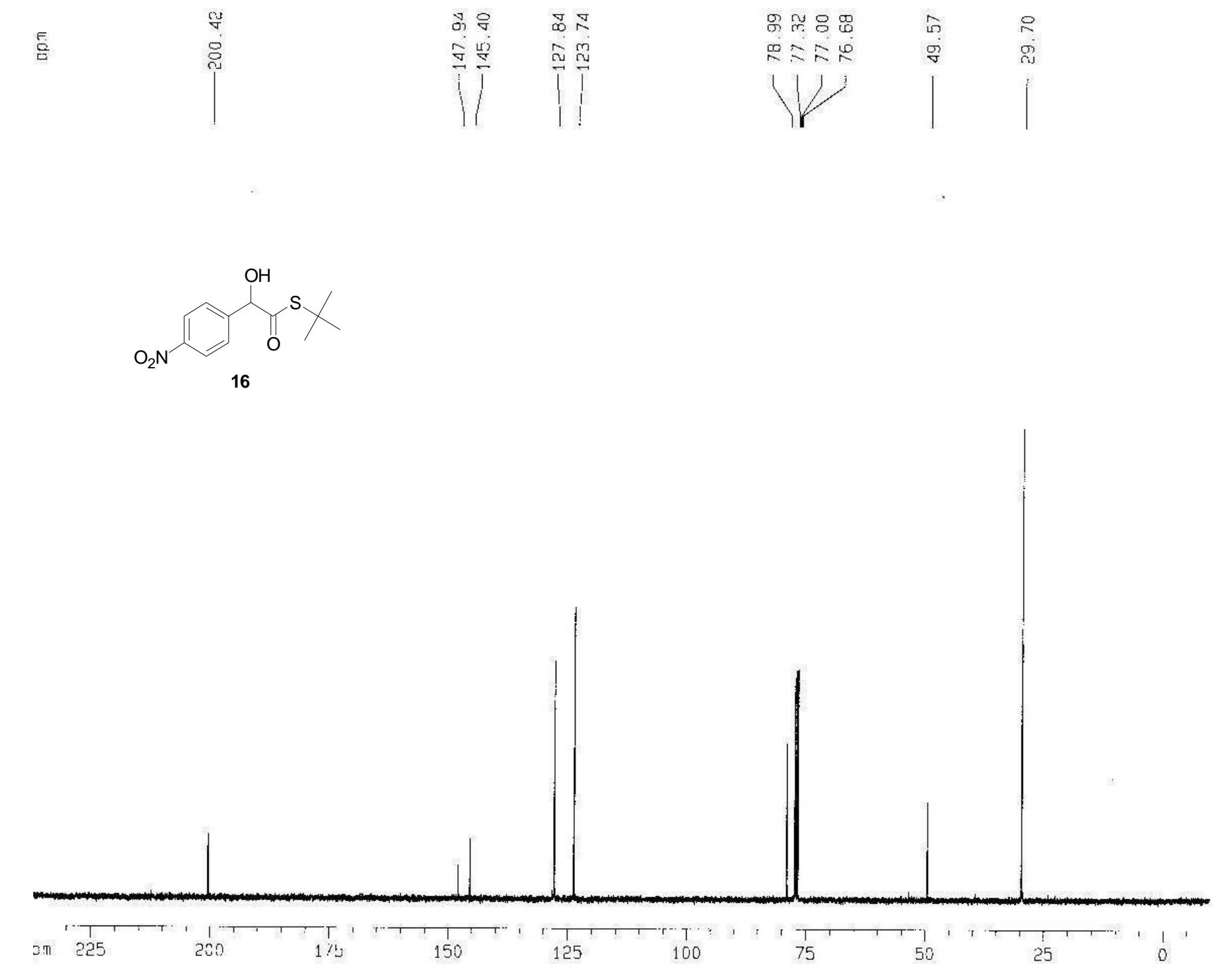

요욤용

器今员

12
Current Lata Parameters

EXNE $\quad$ Säm-HT-Aח 1 tro

JFOCNO

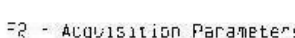

Jite_-

INSTRUY

คULOROG

TD

SOLVE

עS

SWH 25125

F JORES $\quad 0.383387 \mathrm{~Hz}$

AQ $\quad 1.3042162 \mathrm{sec}$

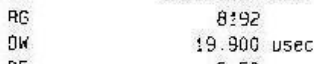

JE $\quad 6.50$ usec

J1

1. $200000005 \mathrm{FeC}$ $0.03000000 \mathrm{sec}$
$0.00002000 \mathrm{sec}$

$==== \pm==$ CHANNEL +1 =s===="

NUC1 $== \pm=E$ CHANNEL $+10= \pm====$

D.1 $\quad 10.80$ usec

SF01 $100.6242995 \mathrm{MHz}$

$======$ CHANNEL $f 2=======$

CPIPAG? waltz210

NuC2 1 1.

$30.03 \mathrm{usec}$
$\mathrm{L} 2 \mathrm{O}$

2L12 $21.63 \mathrm{dE}$

$24.60 \mathrm{de}$

$1399000 \mathrm{~m} M$

$=2$ - Processing parameters

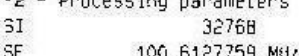

SF $\quad 100.6127759$ Mst

IDSB

$\begin{array}{ll}\mathrm{H} & \mathrm{B} \\ \mathrm{GB} & \mathrm{C}\end{array}$

10 NMA p]nt parameters

ix $\quad 20$ plat a

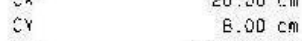

$=1 \mathrm{P} \quad 239.397 \mathrm{pF} . \mathrm{m}$

$=2 \mathrm{P} \quad 24066.35 \mathrm{~Hz}$

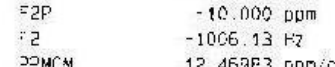

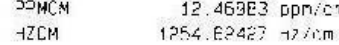


11 spertnum of
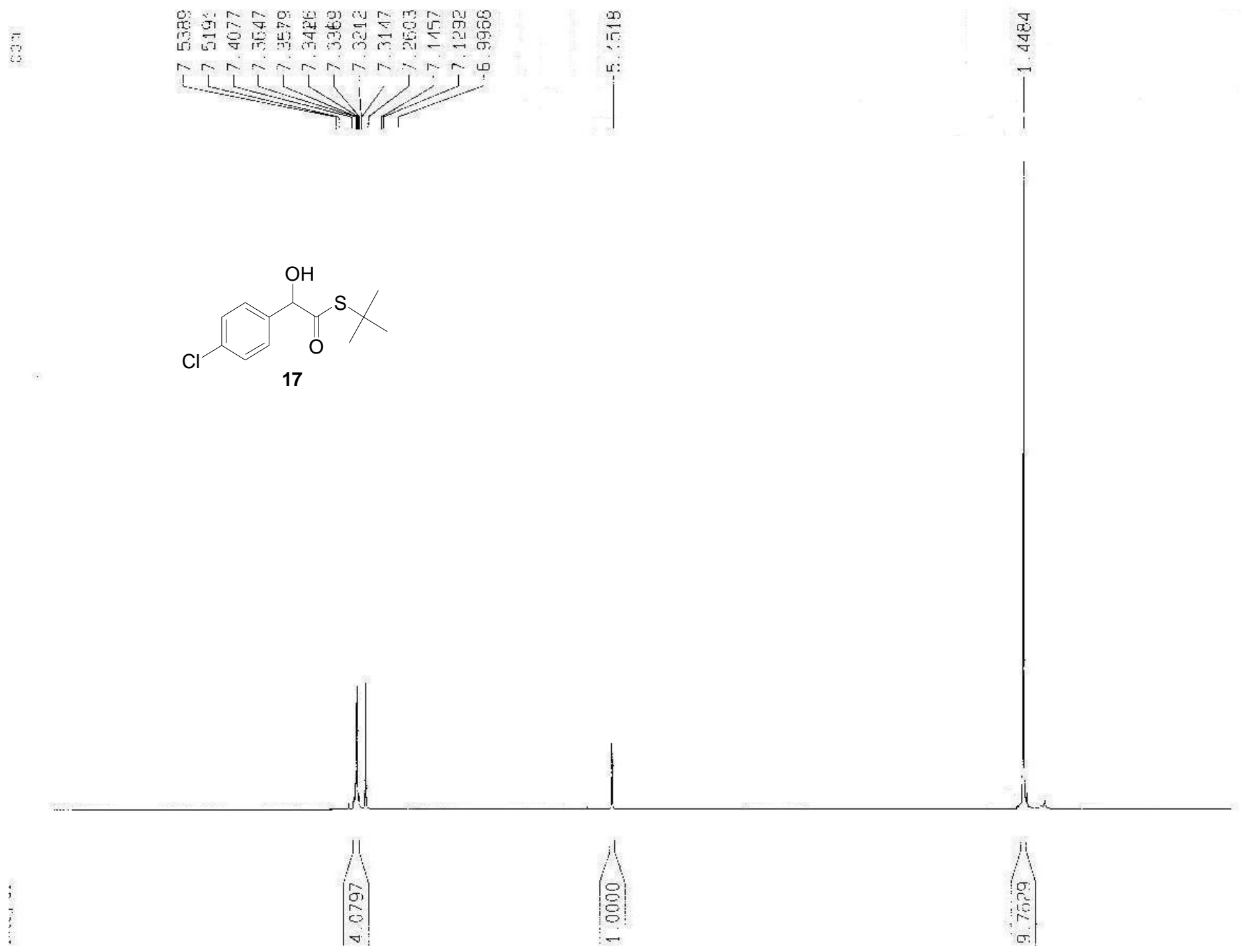

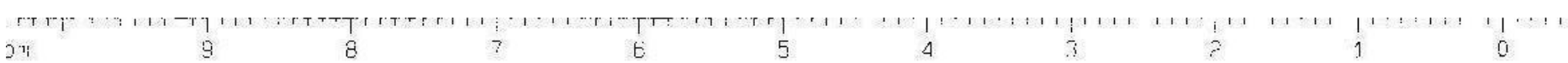

whin

$4+1+1$

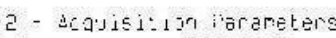

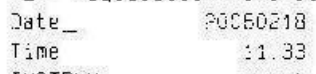

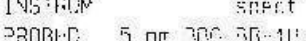

J IPFCE

JJLPFCE 2930

SITLUENT

N3

SWH

ก. $355938 \mathrm{~B} / \mathrm{H}$

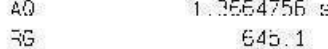

$\begin{array}{rr}70 & 64.1 \\ 3 W^{\prime} & 83.430 \text { usec }\end{array}$

$6.50 \mathrm{Lesp}$

1. $50000000 \mathrm{sLc}$

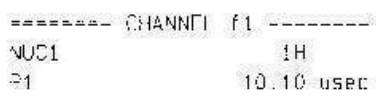

IL: 3.00 แB

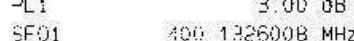

$-z-$ processinc färmielers

SI Proceszing carsmaters

$\mathrm{SF} \quad 4001300095$

'NDH' EM

SSB

$\begin{array}{cc}-\mathrm{B} & -10 \\ \mathrm{~g} & \mathrm{C}\end{array}$

${ }^{2}-100$

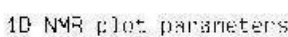

cx $20.00 \mathrm{~cm}$

$=10 \quad 10.50 \mathrm{~cm}$

-1 10 5ioc nom

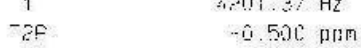

-2 $\quad-2000.07 \mathrm{~Hz}$

(.) 5 5.000 DEm/C

220.0715 ? Hz $2 \mathrm{CH}$ 
है

1)

तो $\mathrm{B}$ 品

品え崖

|

Curreal Data Far'ameters

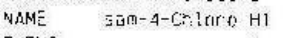

EXPND
כHOCLOO

Fis - Acasisit jum Paramcters

Date

$\begin{array}{ll}\text { TJme } & 22.40 \\ \text { INSTRUM } & \end{array}$

JROBHU 5 Fim ABO SLect

गULPHOS 5 .

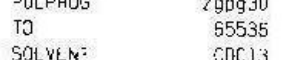

SOLVE

NS

DS $\quad 00$

$\begin{array}{ll}\text { SWH } & 25125 \mathrm{52C} \mathrm{Hz} \\ \text { =IDAES } & 0.363987 \mathrm{~Hz}\end{array}$

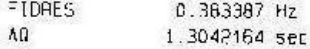

$\begin{array}{ll}\text { RG } & 819 \text { ? } \\ \text { DH } & 19.900 \text { บรง }\end{array}$

DE 5.50 use

$300.0 \mathrm{~K}$

$1.20000005 \mathrm{sec}$

$\begin{array}{ll}\text { J1 } & 0.03000000 \mathrm{set} \\ \mathrm{J12} & 0.00002000 \mathrm{~s} E \mathrm{C}\end{array}$

17

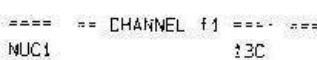

$\begin{array}{lll}\text { NUC1 } & 13 \mathrm{C} \\ \mathrm{D}_{1} & 10.80 \mathrm{usec}\end{array}$

SF01 1.00 a日

IFo1

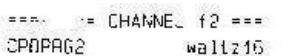

SUC?
TCPD?

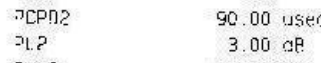

ग1. 93 a

FFG? $400-315002$

$=5$ - Jveressing parameters

5 3I 32768

जF. $100.61 \varepsilon 7700 \mathrm{MH}$ ?

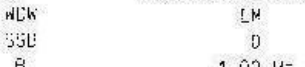

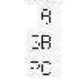

1.40

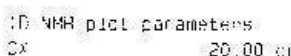

$2 x \quad 20.00 \quad 3.11$

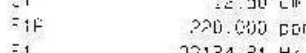

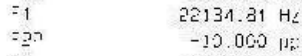

$2 \quad-1005.13 \rightarrow 0$

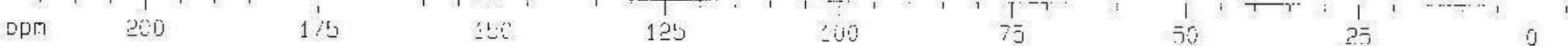

i, TMM

11. 50000 DPAI/III 
镸

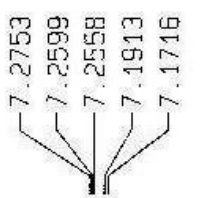

$\mathrm{OH}$

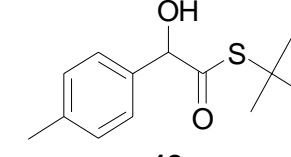

18

$\int^{S}$
H spectrum $\mathrm{ef}$

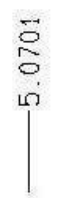

VAML
- XPNO

EXPNC
JROCNC

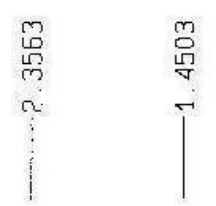

\section{.}

- Acquisitjon Parameter

$\begin{array}{lr}\text { Jate.- } & 20060311 \\ \text { Trme- } & 11.37\end{array}$

INSTRUM spect.

TROBHO $5 \mathrm{~mm}$ BBO BB-1H

$\begin{array}{lr}\text { PULPAOG } & 2930 \\ \text { TD } & 16384\end{array}$

$\begin{array}{ll}\text { TD } & 16384 \\ \text { SOL. YENT } & \text { CDC } 13\end{array}$

$\begin{array}{lr}\text { NS } & 15 \\ \text { DS } & 9\end{array}$

\begin{tabular}{ll} 
DS & \multicolumn{2}{c}{} \\
SWH & $5995.204 \mathrm{~Hz}$
\end{tabular}

FIDRES $\quad 0.36591 \mathrm{H} \mathrm{Hz}$

$\triangle Q \quad 1.3664756 \mathrm{se}$

RG 574.1

$\begin{array}{lr}\text { DW } & 83.400 \text { user } \\ \text { DE } & 6.50 \text { user }\end{array}$

$300.0 \mathrm{~K}$

1. $50000000 \mathrm{sec}$

$======$ CHANNEL $f 1$ =="===="

$\begin{array}{ll}\text { NUC1 } & 1 \mathrm{H} \\ P 1 & 10.10 \text { usec }\end{array}$

$\begin{array}{lr}\text { P1 } & 10.10 \text { USEC } \\ \text { PL1 } & 3.00 \mathrm{dE} \\ \text { SF01 } & 400.132600 \mathrm{MHL}\end{array}$

-? - Proces5ang parameters

SI 16384

SF $\quad 400.130 \% 995 \mathrm{MHF}$

WOW

SSB
$-B$

38

EM

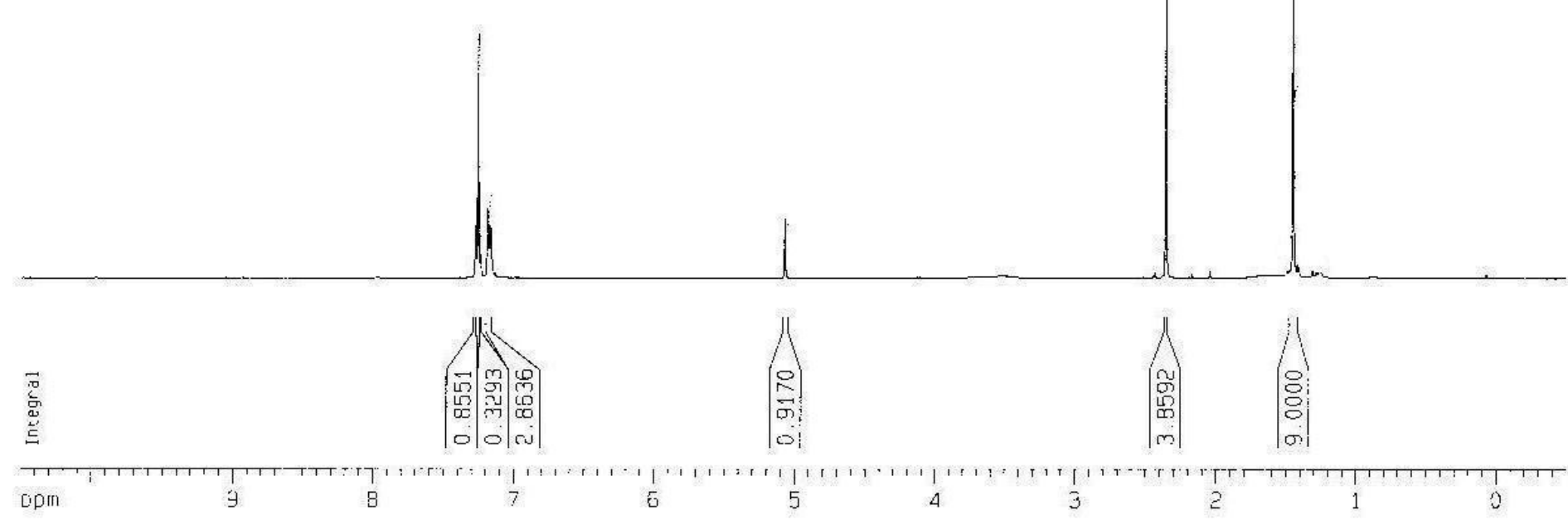

1D NMR plot paraneters

cx $20.00 \mathrm{~cm}$

$10.50 \mathrm{~cm}$

F1 $10.500 \mathrm{ppm}$

$\begin{array}{ll}F 1 & 4201.37 \mathrm{IL} \\ F Z P & -0.500 \mathrm{ggm}\end{array}$

F2 $\quad-200.07 \mathrm{~Hz}$

0. $5500020 \mathrm{~m} / \mathrm{cm}$ 
톨
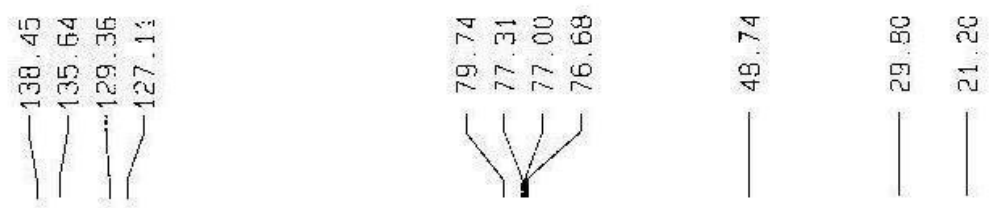

Currerit Uata Parameters

EXPNO

PAOCN]

F2 - Acqu151:10n Parameters

$\begin{array}{lr}\text { Date- } & 20060311 \\ \text { Time } & 17.4 \% \\ \text { The } & \text { rat }\end{array}$

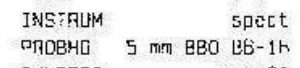

PULPACG 2 290930

$\begin{array}{ll}\text { TO } & \text { ELLVENT } \\ \text { SDC13 }\end{array}$

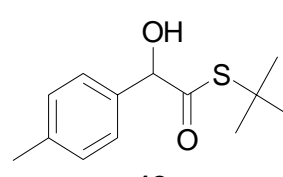

$\mathrm{S}$

18

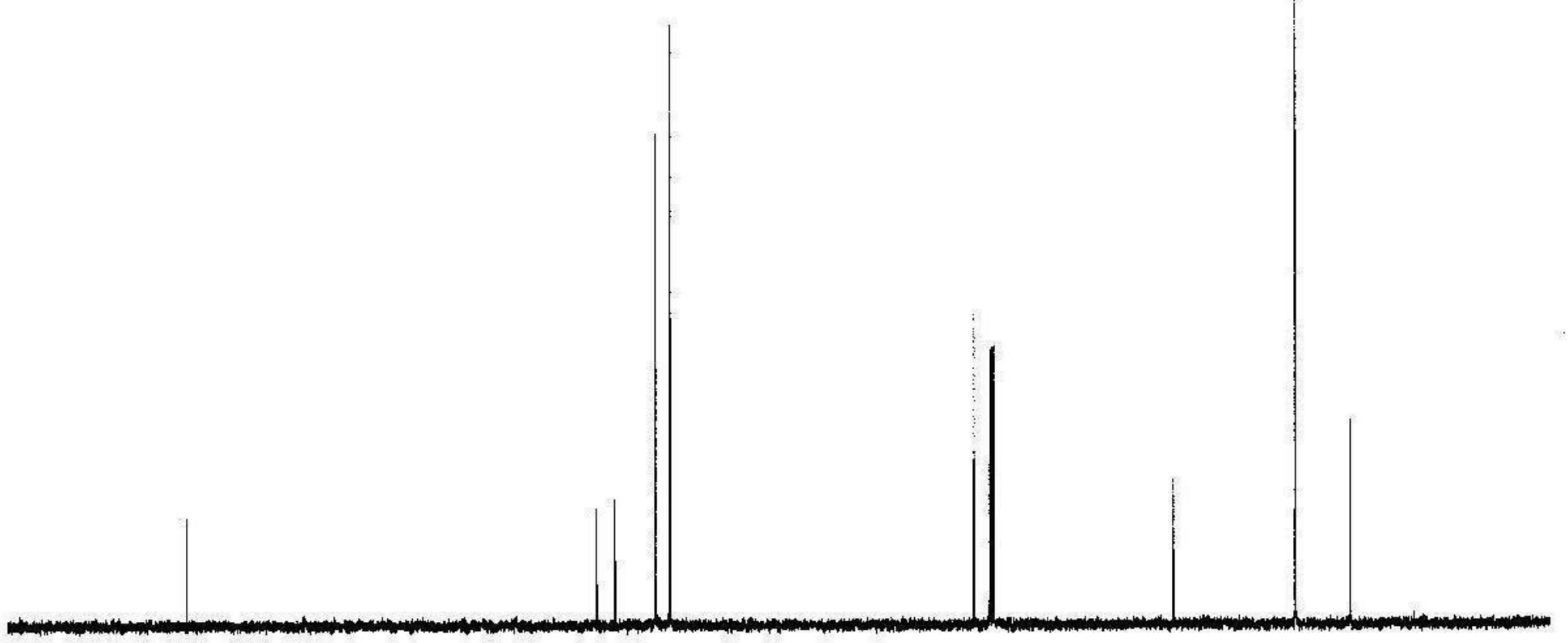

,

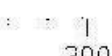

1
200

175

150

123

100

50

i 35

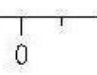

VS

J5 25125 대

$\begin{array}{ll}25125.629 \mathrm{~Hz} \\ =\text { IDRES } & 0.363387 \mathrm{~Hz}\end{array}$

AG $\quad 3.3042164 \mathrm{sec}$

319.900 usec

6.50 usec

$\begin{array}{ll}\mathrm{TE} & 300.0 \mathrm{~K} \\ \mathrm{j} & \therefore .20000005 \mathrm{sec}\end{array}$

0.0000200 sec

.

NUC1 $=$ CHANNEL $\$ 1======-13 \mathrm{C}$

$\begin{array}{lr}01 & 10.60 \mathrm{usec} \\ 7 & 7.00 \mathrm{ag}\end{array}$

5F $01 \quad 100.6242995 \mathrm{MHZ}$

$=z====$ CHANNEL $+2=======$

PAPRG2 waltz15

$\begin{array}{lc}\text { MULC } & 1 \mathrm{H} \\ \text { PCPO2 } & 90.00 \mathrm{usec}\end{array}$

$\begin{array}{lll}\text { PL5 } & 3.00 \mathrm{~dB}\end{array}$

PL13 13.030

SF Qc $\quad 400.1319000 \mathrm{MHZ}$

F2 - Iracessing parameters

SF $\quad 100.6127767 \mathrm{MHz}$

HOW

$\therefore$

39

NHR plot parameters

Ex $20.00 \mathrm{~cm}$

$\begin{array}{lr}2 Y & 12.50 \mathrm{~cm} \\ =1 \mathrm{~cm} & 230.000 \mathrm{pPm}\end{array}$

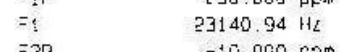

$\begin{array}{ll} & =5 \mathrm{P} \\ =2 & -10.000 \mathrm{com}\end{array}$

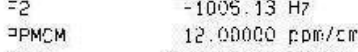

12C4 1297. $35327 \mathrm{~Hz} / \mathrm{Lm}$ 
H spectrum of

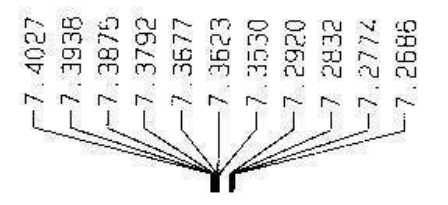

邑

$\mathrm{Cl} \quad \mathrm{OH}$ $\int_{0}^{\mathrm{S}}$

19

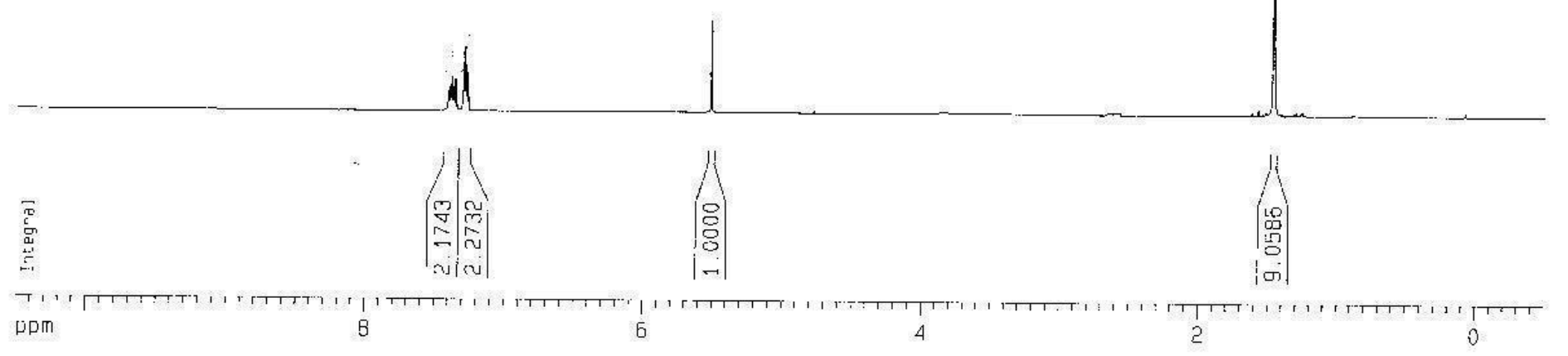

$\begin{array}{lr}\text { NAME } & \text { 5am-5 C1-AT } \\ \text { EXFNOS } & \vdots \\ \text { DROCNC } & 1\end{array}$

$=2$ - Acquisutuen parameters

Daie__ 2006:04?66

ING $12.2 \%$

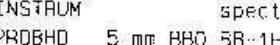

PUI PROS
TD

SOLVENT

v5
is

IS

$.995 .004 \mathrm{~Hz}$

FIURES $0.76591 \mathrm{~B} \mathrm{~Hz}$

$40 \quad 3.366475 t=5$

JE

:E

31

114

5. 50 บะอC

$300.0 \mathrm{~K}$

150000003 5e:

$-======$ CHANNEL
NHLC1
PHC

$\begin{array}{lc}\text { NHLC1 } & 1 \mathrm{H} \\ \text { P1 } & 10.10 \text { usec }\end{array}$

D:1 $3.00 \mathrm{JB}$

$400.1326002 \mathrm{MH}$

=2 - Processing parameters

Si $\quad 16304$

SF 400.1300091 MIH

WDW $39 \mathrm{EM}$

$\begin{array}{lr}3 S B & 0 \\ i B & 0.10 \mathrm{~Hz}\end{array}$

CB

10 nimp plct parameiers

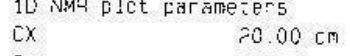

$\begin{array}{ll}\mathrm{CX} & 20.00 \mathrm{~cm} \\ \mathrm{CY} & 10.50 \mathrm{~cm}\end{array}$

$=1 \mathrm{P} \quad \therefore 0.500 \mathrm{ppm}$

$=1 \quad 4261.37 \mathrm{H}$

$\begin{array}{ll}-0.500 \mathrm{pFm} \\ \mathrm{Fe} & -2000.06 \mathrm{~Hz}\end{array}$

PPMCM

$\mathrm{HZCM} \quad 220.0 \% 150 \mathrm{HLT} / \mathrm{Cm}$ 
C13 spertrum of

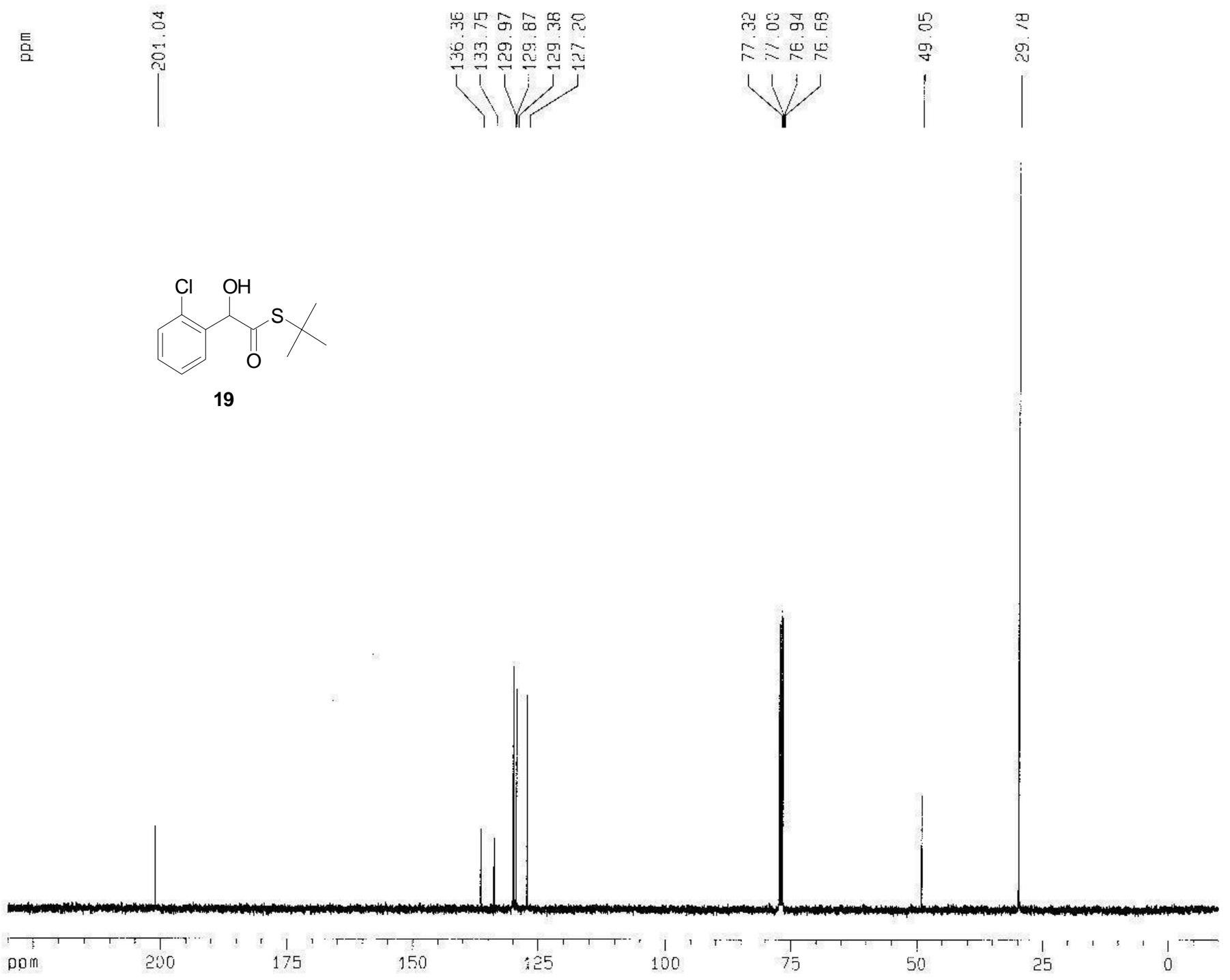

Gurrent Data Darameter

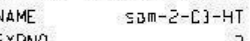

EXPNa

$\because 2$ - Acquisjtion Parameters

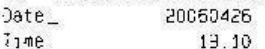

ine

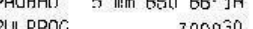

790930
TD

sis

0
$5 \mathrm{WH}$$\quad 25125.629 \mathrm{~Hz}$

HULLS $\quad 0.383397 \mathrm{~Hz}$

765

$\begin{array}{rr}J \mathrm{~W} & 19.900 \text { usec } \\ \mathrm{JE} & 6.50 \text { usec }\end{array}$

$5.50 \mathrm{usec}$
$300.0 \mathrm{~K}$

$31 \quad 1.20000005 \mathrm{sec}$

$\begin{array}{lll} & 112 \quad 0.00000000 \mathrm{sec} \\ 0.00002000 & 5 \mathrm{ec}\end{array}$

$== \pm= \pm=$ CHAMNEL $f 1 \quad \ldots .$.

MUC1 C. HAFAEL I1 $13 \mathrm{C}$

$\begin{array}{lr}\text { P1 } & 10.00 \mathrm{dse} \\ \text { PL1 } & 1.00 \mathrm{~dB} \\ \text { SF01 } & 100.6242995 \mathrm{MHz}\end{array}$

$== \pm== \pm=$ CHANNFI f $2======$
CPDPRG?

WL?

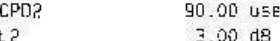

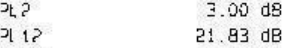

गL13 24.80 dB

=2 - Processing zarigmeters

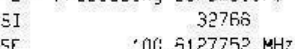

ST $\quad$ :OC. $5127752 \mathrm{MHz}$

SSD

$\mathrm{B}$
$\mathrm{SB}$

15 N4A plot parameters
CX. 20.00 of .00
.50
$\mathrm{~cm}$ pon 
$H$ تpectrum of
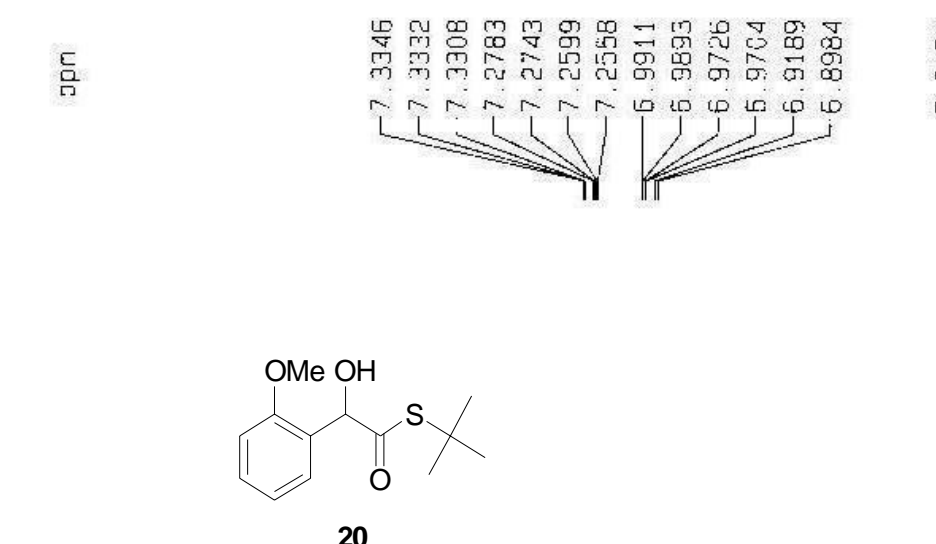

20
$\mathrm{OMe} \mathrm{OH}$

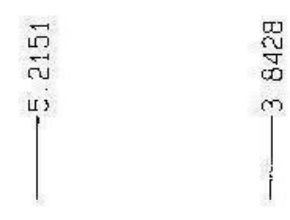

गु

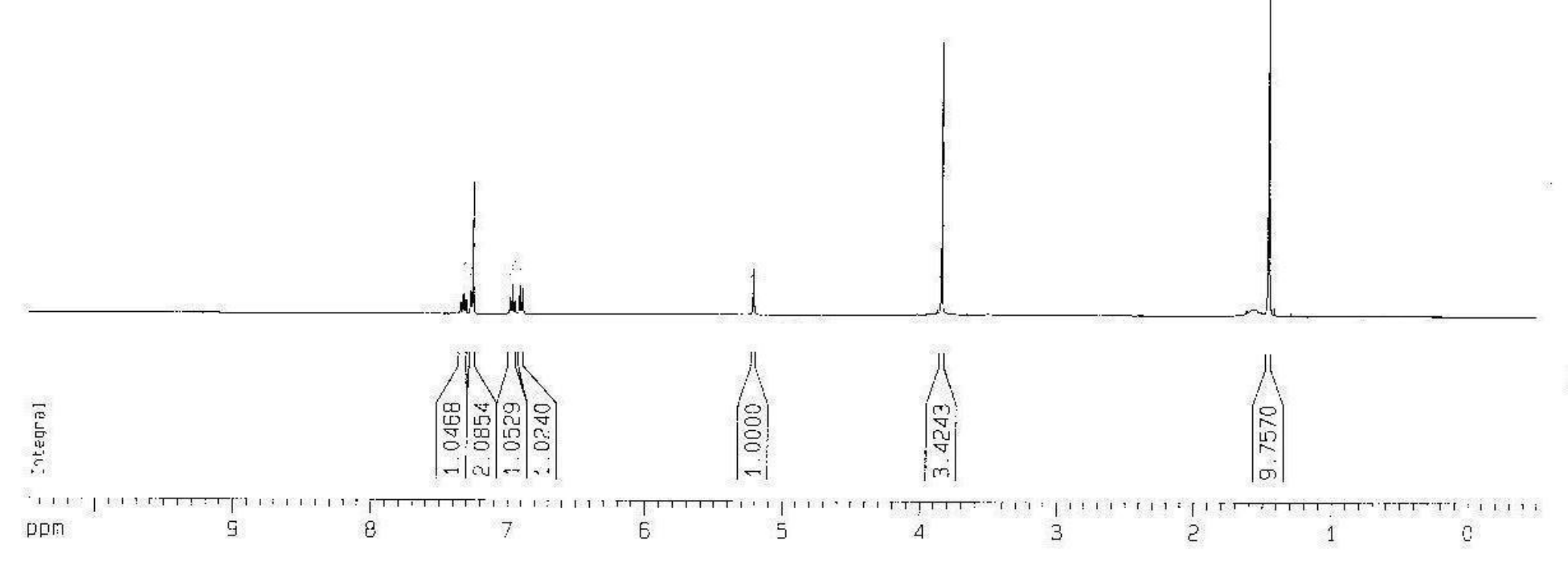

VAME ग-DME-4T-H :

EXPNO

F2 - Acqujsition Paraneters

Date _ 200602?6

та те 19.52

INSTFUM Spect

PROBHO $5 \mathrm{~mm}$ BBO BQ-1H

PU:PAOG Z $\quad$ ZG30

TOL

N5
JS

SWH $\quad 5995.204 \mathrm{~Hz}$

$=$ IDRES $\quad 0.365915 \mathrm{~Hz}$

A0 $\quad 1.3654756 \mathrm{~Hz}$

RG $1.3664756 \mathrm{5ec}$

DWW B3. 51200 use:

6.50 usec

$\begin{array}{cc}\text { th } & 300.0 \mathrm{~K} \\ \mathrm{~J} & 1.50000000 \mathrm{SPC}\end{array}$

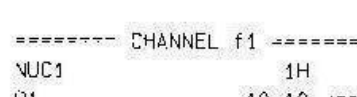

P1 10.10 usec

$\begin{array}{lr}\text { Fl } 1 & 3.00 \mathrm{~dB} \\ \text { SFO1 } & 400.1326008 \mathrm{MHz}\end{array}$

F2 - Prncessing paranetcrs

$5:-10334$

$\begin{array}{lr}\text { SF } & 400.1300095 \mathrm{MHz}\end{array}$

W.W EM

-B $0.10 \mathrm{~Hz}$

$\mathrm{OC} \quad 0$

1D NaAR plot paramete?'s

cx $20.00 \mathrm{~cm}$

cY $10.50 \mathrm{~cm}$

$=1 \mathrm{~F} \quad 10.50 \mathrm{C} \mathrm{FDm}$

$\begin{array}{lr}-1 & 40.501 .37 \mathrm{~Hz} \\ -2 p & -0.5000 \mathrm{OPH}\end{array}$

$\begin{array}{ll}-2 & -0.500 \mathrm{DPM} \\ -? & -200.07 \mathrm{~Hz}\end{array}$

$\begin{array}{ll}3 P \mathrm{PHCM} & 055000 \mathrm{Dm} / \mathrm{cm} \\ \mathrm{HZCM} & 220.07152 \mathrm{~Hz} / \mathrm{cm}\end{array}$ 


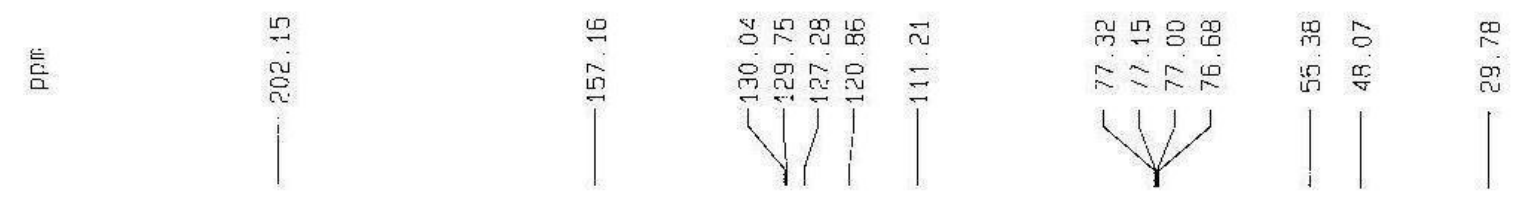

$\mathrm{OMe} \mathrm{OH}$

นIJ spelc. "uII uT

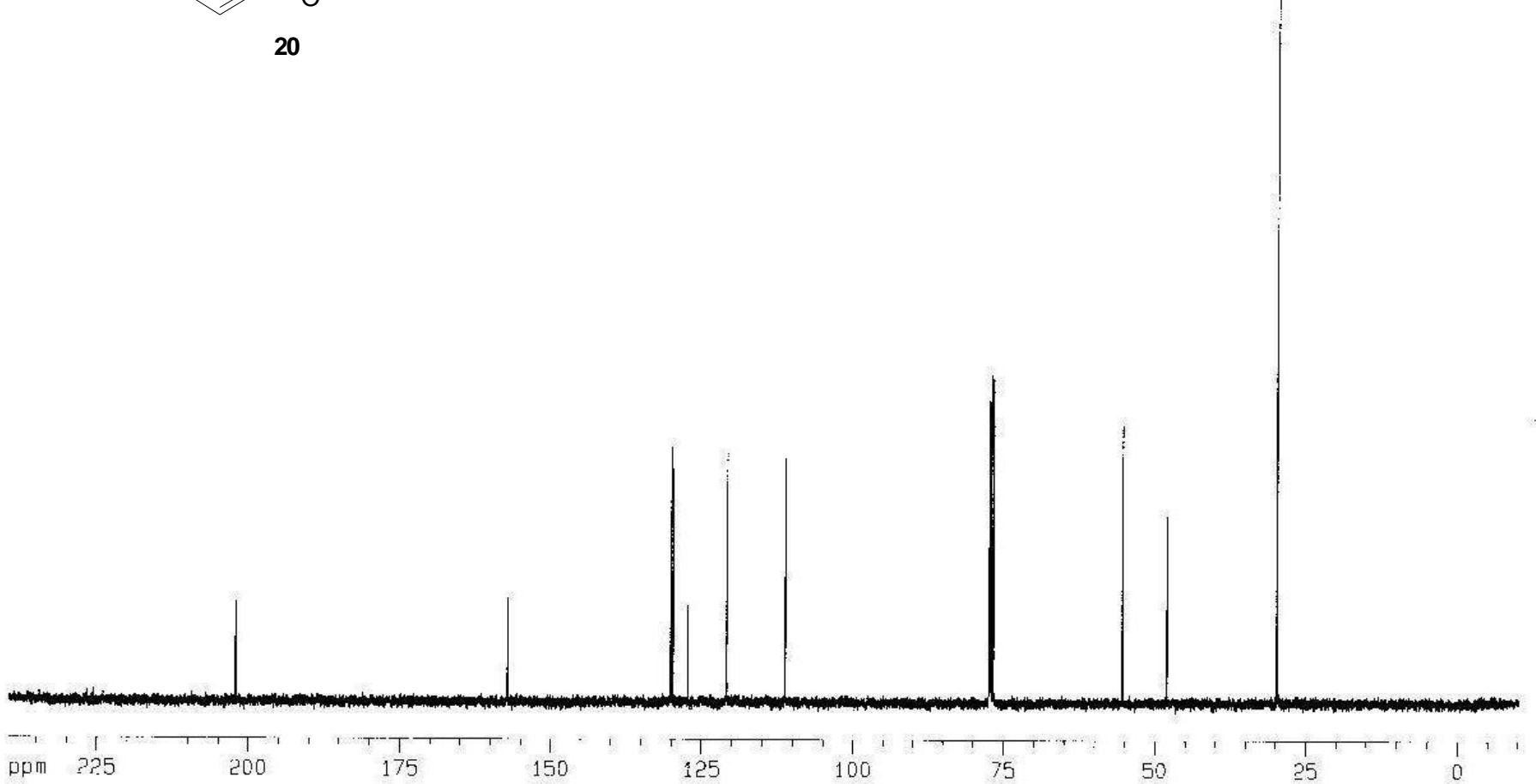

$\top$
175
150
100

25

1
0

$$
\begin{aligned}
& \text { Current Data Par'ameters } \\
& \text { PROCKn } \\
& \text { Time }
\end{aligned}
$$

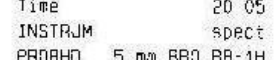

$$
\begin{aligned}
& \text { PULPROG } \\
& \begin{array}{ll}
\text { IO } & 65536 \\
\text { SOLVENT } & \quad 0.513
\end{array} \\
& \text { is }
\end{aligned}
$$

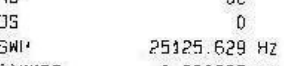

$$
\begin{aligned}
& \begin{array}{ll}
0.399387 \mathrm{~Hz} \\
1.3042154 \mathrm{sec}
\end{array}
\end{aligned}
$$

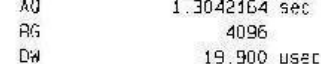

$$
\begin{aligned}
& \begin{array}{lr}
\text { Did } & 19.900 \text { usen } \\
\text { DE } & 6.50 \text { usee }
\end{array} \\
& 300.0 \mathrm{~K} \\
& \begin{array}{cc}
-\mathrm{B} & 1.00+z \\
3 \mathrm{~B} & 0 \\
\mathrm{JC} & 1.40
\end{array}
\end{aligned}
$$

10. NMB plot parameters

cx $\quad 20.00 \mathrm{~cm}$ $\begin{array}{lr}\mathrm{Cr}_{19} & 11.00 \mathrm{~cm} \\ \mathrm{Cl}^{2} & 239.374 \mathrm{ppm}\end{array}$ $\begin{array}{ll}{ }_{1} & 24084.06 \mathrm{~Hz} \\ =2 p & -10.352\end{array}$ $\begin{array}{ll}-2 & -16.352 \mathrm{OEF} \\ -\bar{c} & -1041.57 \mathrm{~Hz}\end{array}$

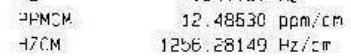


H spectrum of
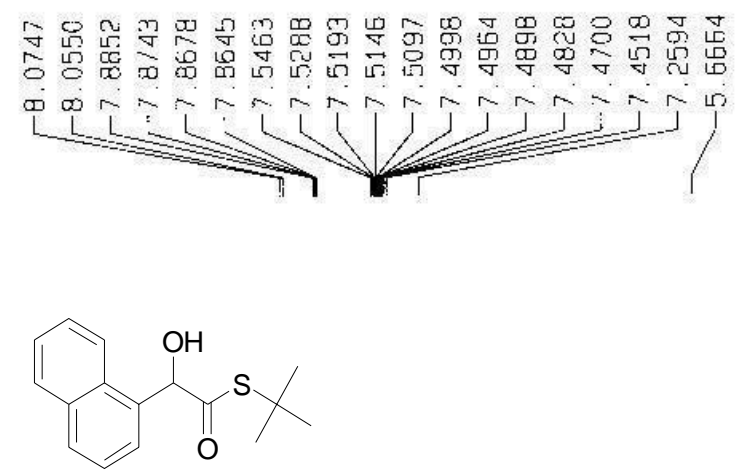

21

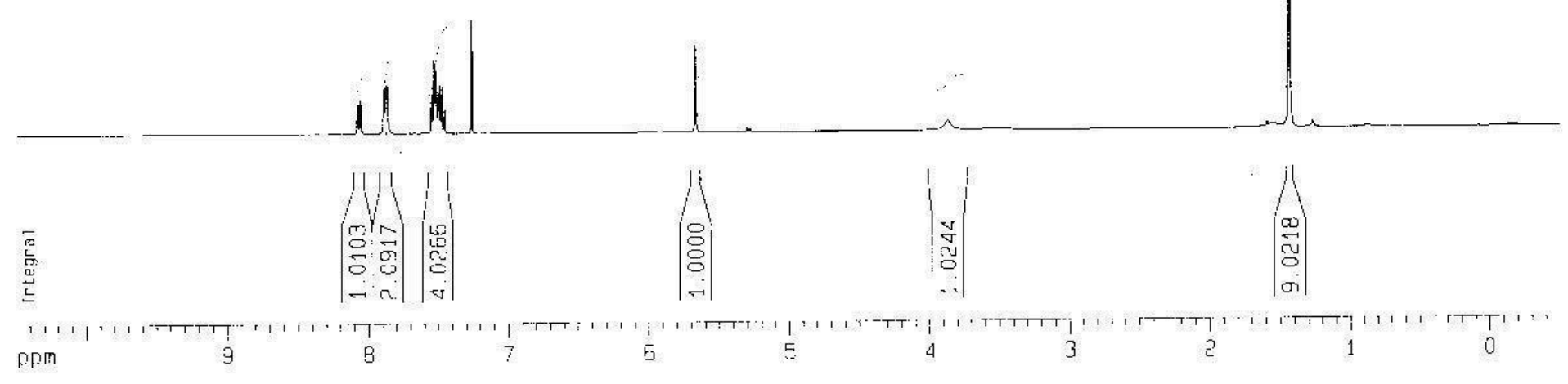

:SAML
EXPND

PHOCNO

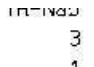

z - acquisition Parameters

Jate_ 2005032?

Time $\quad 16.57$

INSTRUM 5 DEec.

PRCPHLO 5 mm BBO BB-1

OULP

TD 1338

$\begin{array}{lr}\text { SOI_VENT } & \text { CDL: } 23 \\ \text { NS } & 60\end{array}$

JS

SWH $5595.204 \mathrm{H}+12$

=IDRES $\quad 0.365918 \mathrm{~Hz}$

AQ $\quad 1.3664756 \mathrm{sec}$

AG 512

CW 23.400 $155 \mathrm{EC}$

JE 6.50 use

$\begin{array}{lr}\text { TE } & 300.0 \mathrm{~K} \\ \mathrm{J1} & 1.5 \mathrm{J000000} \mathrm{sec}\end{array}$

$==-====$ [HANNEI $f 1==-=-+=$

NUC1 $1 \mathrm{H}$

P1 10.10 ᄂ5e

ㅁ1 $3.00 \mathrm{~dB}$

SFO: $400.132600 \mathrm{~B} \mathrm{MHZ}$

-2 - Prucessing parameters

ST 16384

$\mathrm{SF} \quad 400.1300099 \mathrm{MHz}$

WOW

sSe

LB

EM

0
$0.10 \mathrm{~Hz}$

$0.10 \mathrm{~Hz}$
0

$\triangle$

1.00

10 NMR plot parancters

Ex 2000 c.m

$\begin{array}{lr}\text { EY } & 15.00 \mathrm{c.m} \\ \text { EjF } & 10.500 \mathrm{F.m}\end{array}$

F1 $4201.37 \mathrm{~Hz}$

FEF $2500 \mathrm{pDm}$

$-25007 \mathrm{~Hz}$
$-5 \mathrm{H}$

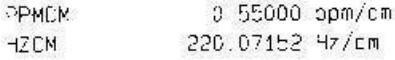




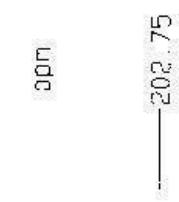

을

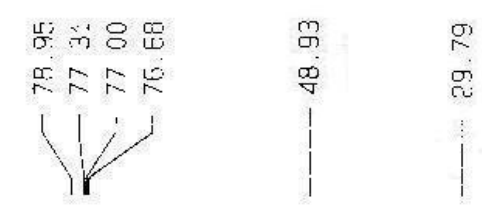

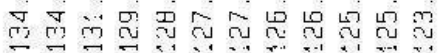

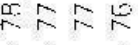

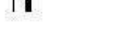

$\mathrm{OH}$ $\mathrm{s}$

21

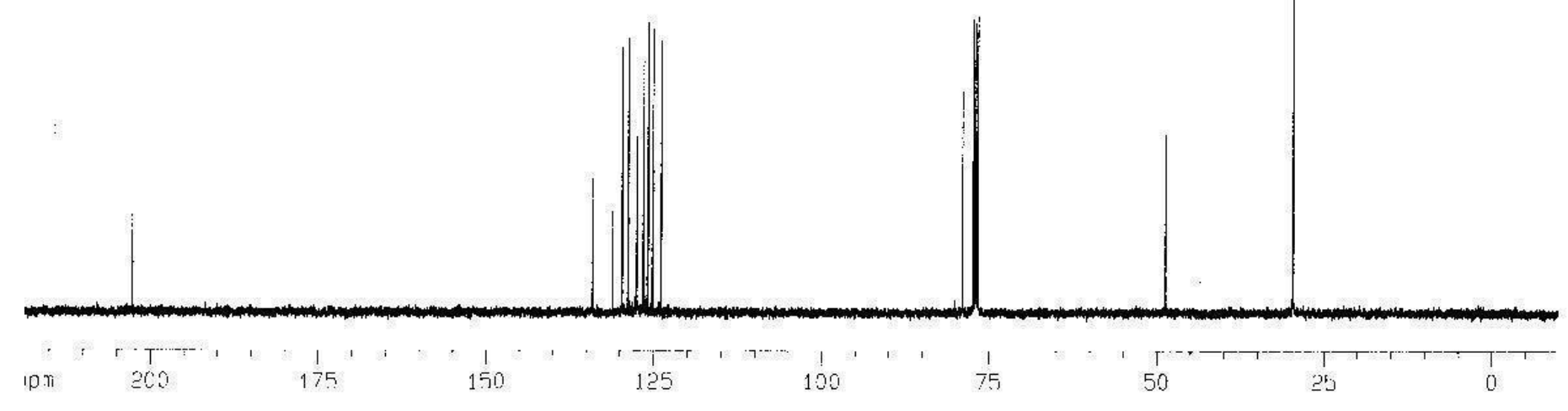

Uurrent Da:o Parameters

JAME 1 . Nan-K!- D

XXPNC

भIDT:

=2 - acosisit:on Parameters

jate. 200603?

$11 \pi e$ 15. 45

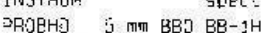

PII. FROI: $\quad 290030$

T)

$\begin{array}{lr}\text { SOI VFNT } & \mathrm{COCLI} \\ \mathrm{VS} & 140\end{array}$

JूH $\quad 25125629 \mathrm{~Hz}$

2. $0.393397-12$

$\begin{array}{ll}40 & 1.3042154 \mathrm{SEC} \\ 76 & 4095\end{array}$

Din 19.905 ÿec

$6.50 \mathrm{l}$ user
$300.0 \mathrm{~K}$

20000005 sec

$111 \quad 0.03000000 \mathrm{sec}$

Jis

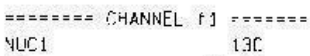

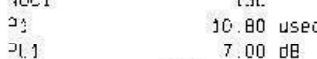

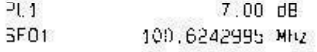

$== \pm===-$ CHANNEI. f? $= \pm x= \pm$,

JPIPFG2 waltz15

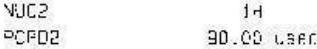

PL2 3.00 तR

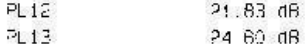

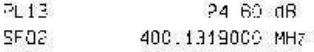

$=\bar{c}$ - Processing parameters

51 32768

WEW

$\hat{S} \mathrm{~B}$

33

10. NMA bolot parareters

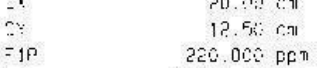
$\begin{array}{ll}11 & 22: 34.61 \mathrm{~Hz} \\ \therefore \mathrm{H} & -30.000 \mathrm{pm}\end{array}$ $=21506: 3 \mathrm{~mL}$

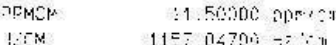


1. ch:et.". pt

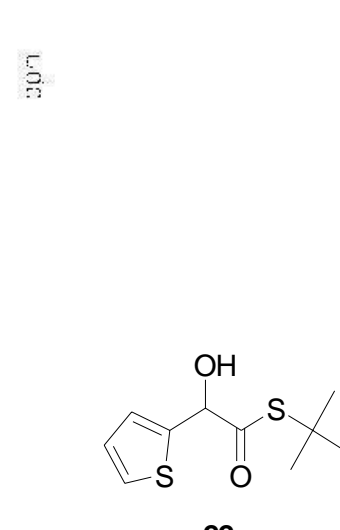

22

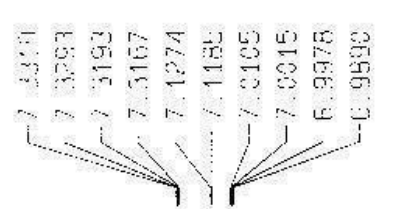

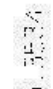

:

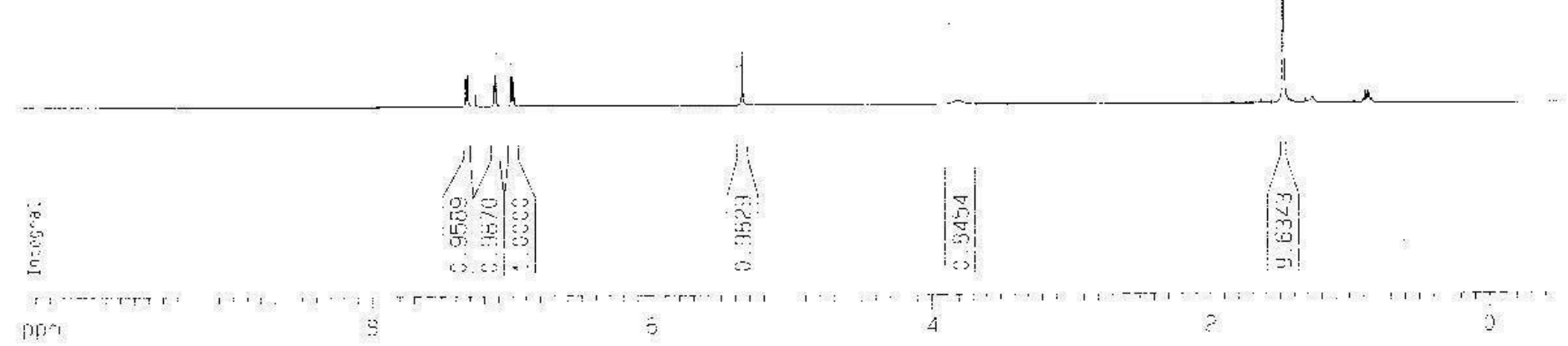

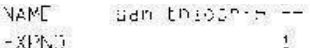

sFocvo

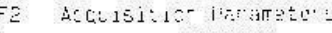

wt? - rotis

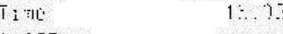

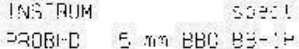

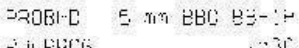

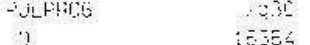

3 HFNT

15

अव

$-19+3=5 \quad 3.365015-12$

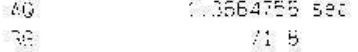
\& 1 is 5 E. 50 LeE

i: $\quad \therefore \quad 300$

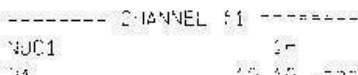

$=11$ a

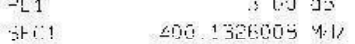

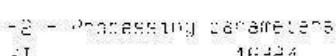

II AN: MOOOS:

WD

$3-4$
3

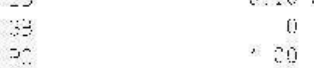

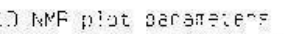

$x$ on

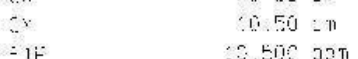

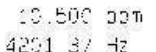
- 4. $-9(10.1) \div 112$

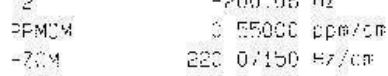




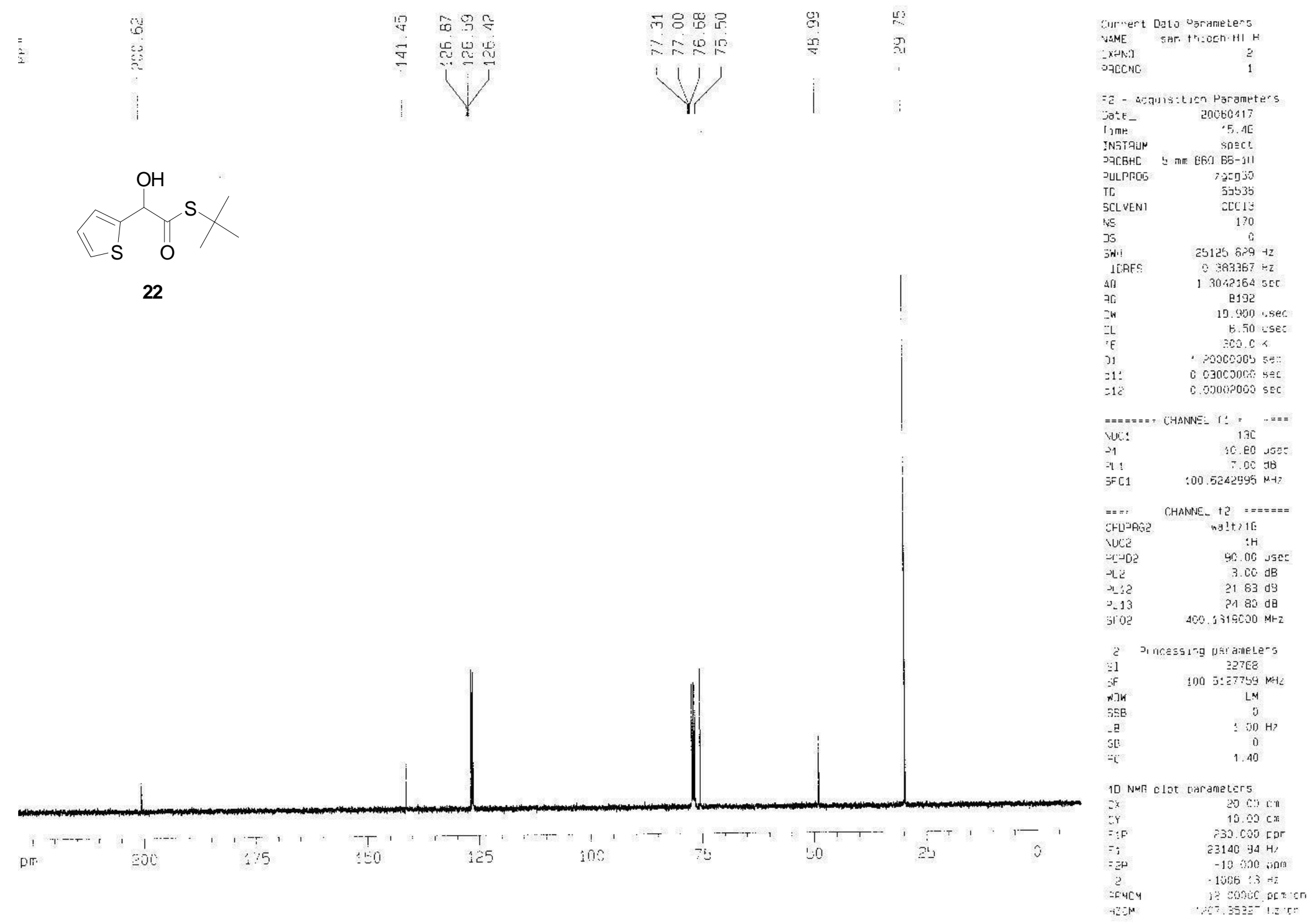



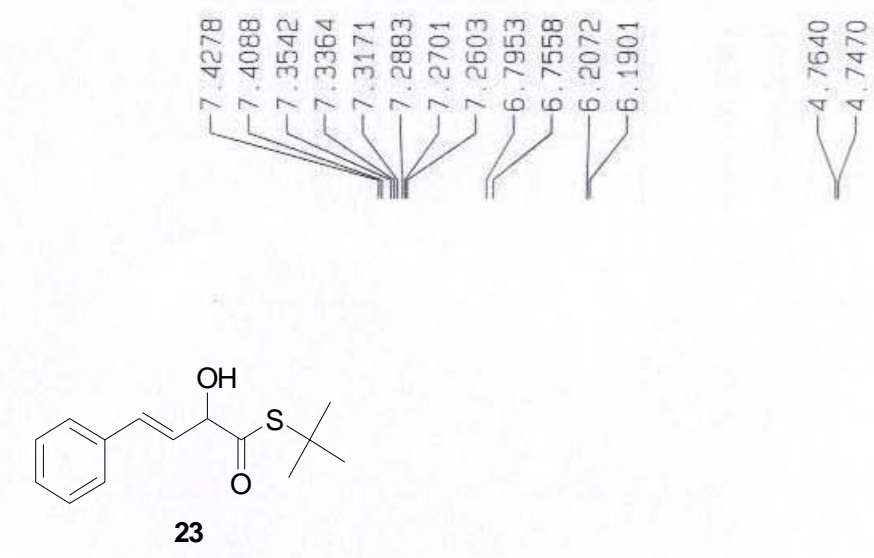

23

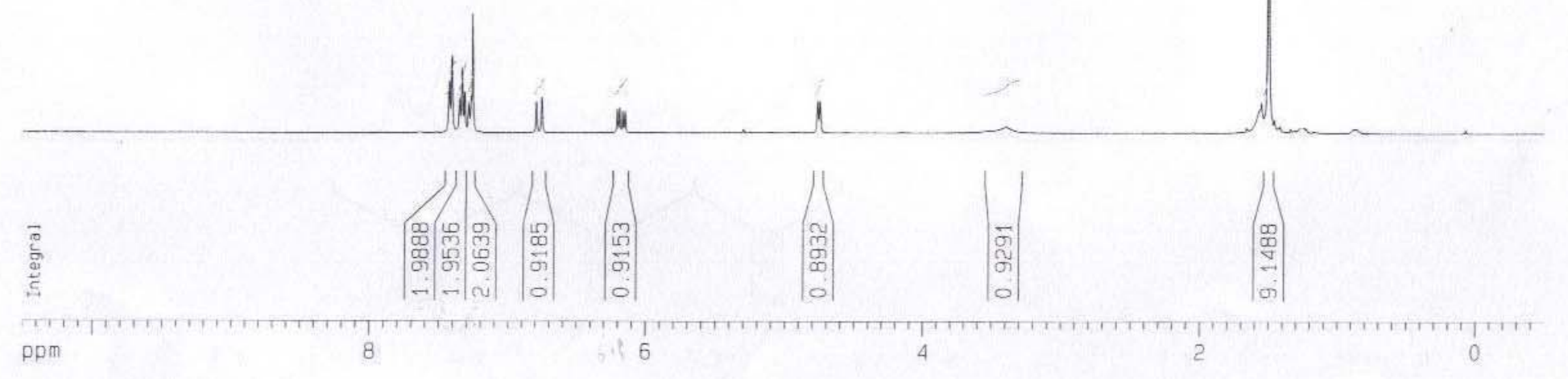

NAME

sam-cinna $H T$

EXPNO

1
1

F2 - Acquisition Parameters

Date_ 20060408

Time $\quad 18.45$

INSTRUM
PROBHD $5 \mathrm{~mm}$ BBOCt
$\mathrm{BB}-1 \mathrm{H}$

PROBRD 5 mIn BBO BD-1H

TO 2930

SOLVENT COC13

NS

$\begin{array}{lr}\text { DS } & 0 \\ \text { SWH } & 5995.204 \mathrm{~Hz}\end{array}$

-IDRES $\quad 0.365918 \mathrm{~Hz}$

AQ 1. $3664756 \mathrm{sec}$

$\begin{array}{lr}R G & 406.4 \\ D W & 83.400 \text { use }\end{array}$

DE $\quad 6.50$ usec

$\begin{array}{lr}\text { TE } & 300.0 \mathrm{~K} \\ \text { D1 } & 1.50000000 \mathrm{sec}\end{array}$

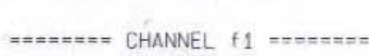

NUC1 $1 \mathrm{H}$

$\begin{array}{lr}\text { P1 } & 10.10 \text { usec } \\ \text { PL1 } & 3.00 \mathrm{~dB}\end{array}$

SF01 $400.1326008 \mathrm{MH2}$

2 - Processing parameters

SI 16384

SF $\quad 400.1300088 \mathrm{MHz}$

$\begin{array}{lr}\text { WDW } & \text { EM } \\ \text { SSB } & 0\end{array}$

LB $\quad 0.10 \mathrm{~Hz}$

$\begin{array}{lr}G B & 0 \\ P C & 100\end{array}$

NMR plot parameters.

cX $20.00 \mathrm{~cm}$

CY $\quad 10.50 \mathrm{~cm}$

F1P $\quad 10.500 \mathrm{ppm}$

F1 $4201.37 \mathrm{~Hz}$

$\begin{array}{ll}-25 & -0.500 \mathrm{ppm}\end{array}$

$-200.06 \mathrm{~Hz}$

$\begin{array}{ll}0.55000 \mathrm{ppm} / \mathrm{cm} \\ \mathrm{HZCM} & 220.07150 \mathrm{~Hz} / \mathrm{cm}\end{array}$ 


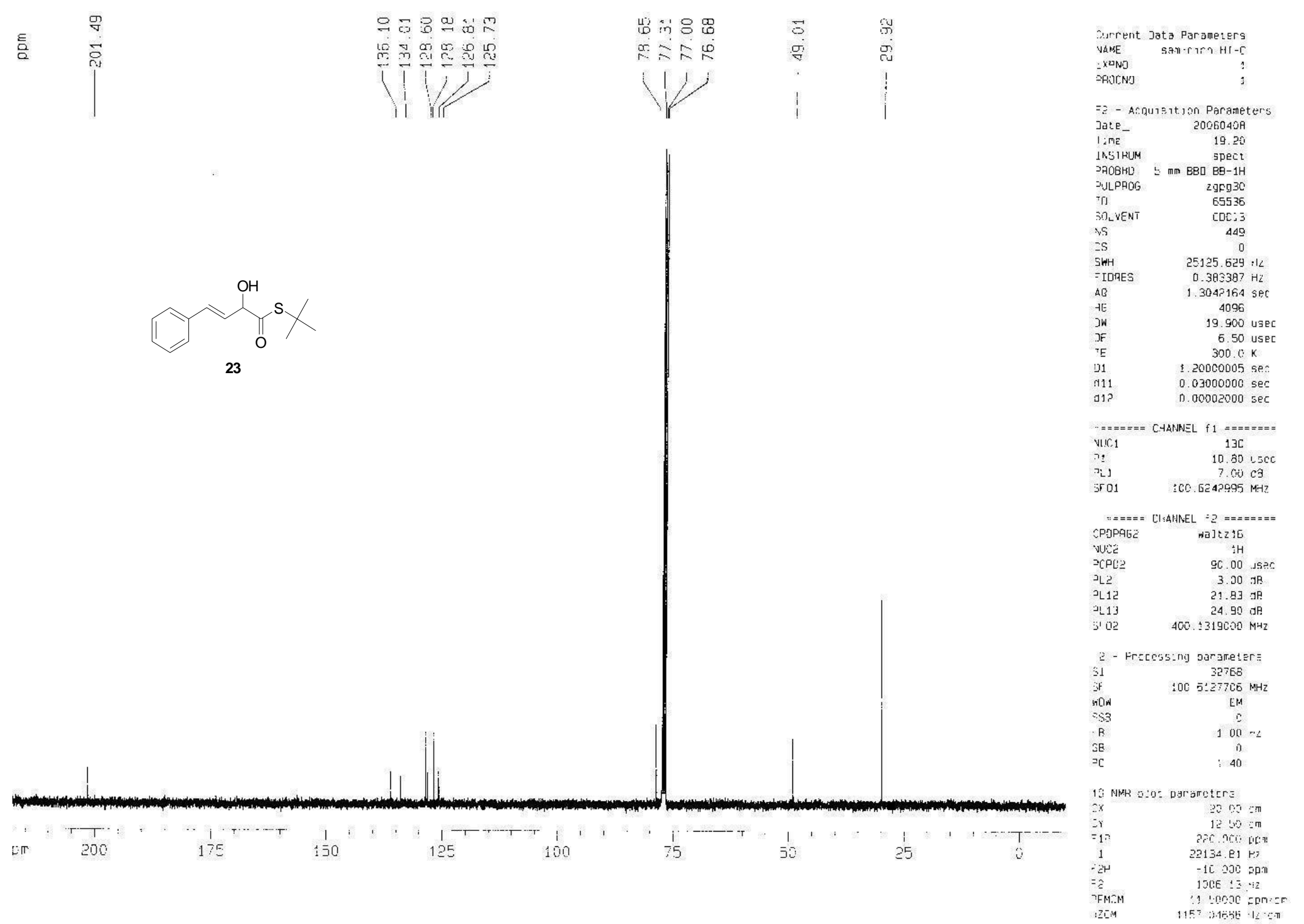


$H$ spectrum of

\section{言}

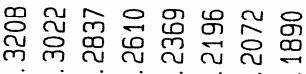
过雀

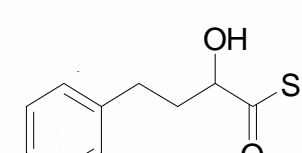

24
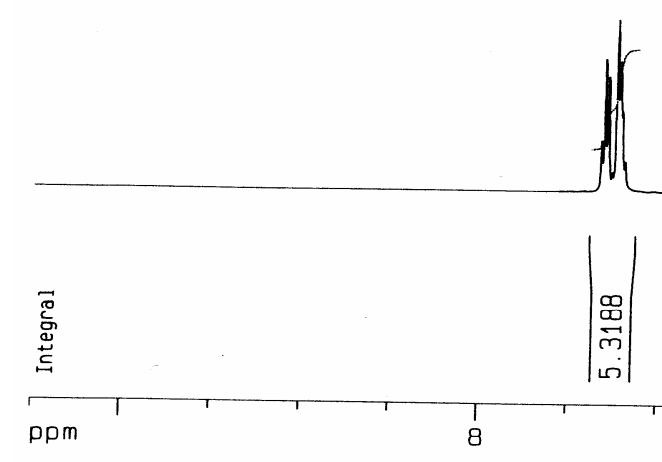

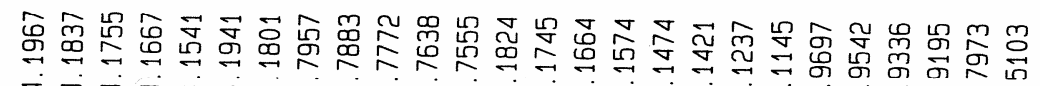

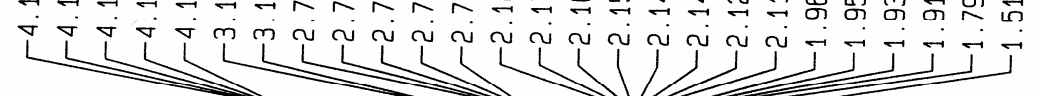

Current Data Parameters NAME

EXPNO

F2 - Acquisition Parameters

Date_ 20060914

Time 17.38

INSTRUM spect PROBHD $5 \mathrm{~mm} B B O \mathrm{BB}-1 \mathrm{H}$

$\begin{array}{lr}\text { PULPROG } & 29 \\ \text { TD } & 16384\end{array}$

SOLVENT

NS

SWH $\quad 00$

$\begin{array}{ll}5995.204 \mathrm{~Hz} \\ \text { FIDRES } & 0.365918 \mathrm{~Hz}\end{array}$

$A Q \quad 1.3664756 \mathrm{sec}$

RG $\quad 228.1 \mathrm{sec}$

$\begin{array}{lr}\text { DW } & 83.400 \text { usec } \\ \text { DE } & 6.50 \text { usec }\end{array}$

TE $\quad 298.6 \mathrm{~K}$

$\begin{array}{ll}\text { D1 } & 1.50000000 \mathrm{sec} \\ \text { MCREST } & 0.00000000 \mathrm{sec}\end{array}$

MCREST $\quad 0.00000000 \mathrm{sec}$

$==== \pm==$ CHANNEL f1 $===2=0$

$==== \pm==$ CHANNEL $f 11====== \pm=$
NUC1 $1 \mathrm{H}$

$\begin{array}{ll}\mathrm{P}_{1} & 7.50 \text { usec } \\ \mathrm{PL} 1 & 23.00 \text { dB }\end{array}$

$\begin{array}{lr}\text { PL1 } & 23.00 \mathrm{~dB} \\ \text { SF01 } & 400.1326008 \mathrm{MHz}\end{array}$

F2 - Processing parameters

SI 16384

SF $\quad 400.1300088 \mathrm{MHz}$

SSB

LB

PC

EM

$1.00 \mathrm{~Hz}$

1.00

1D NMR plot parameters

CX $20.00 \mathrm{~cm}$

$\begin{array}{ll}\mathrm{Cr} & 15.00 \mathrm{~cm}\end{array}$

F1P $\quad 10.500 \mathrm{ppm}$

F1 $\quad 4201.37 \mathrm{~Hz}$

$\begin{array}{ll}F_{2} P & -0.500 \mathrm{ppm} \\ \mathrm{F} 2 & -200.06 \mathrm{~Hz}\end{array}$

PPMCM $\quad-200.06 \mathrm{~Hz}$

HZCM $\quad 0.55000 \mathrm{ppm} / \mathrm{cm}$ 
C13 spectrum of
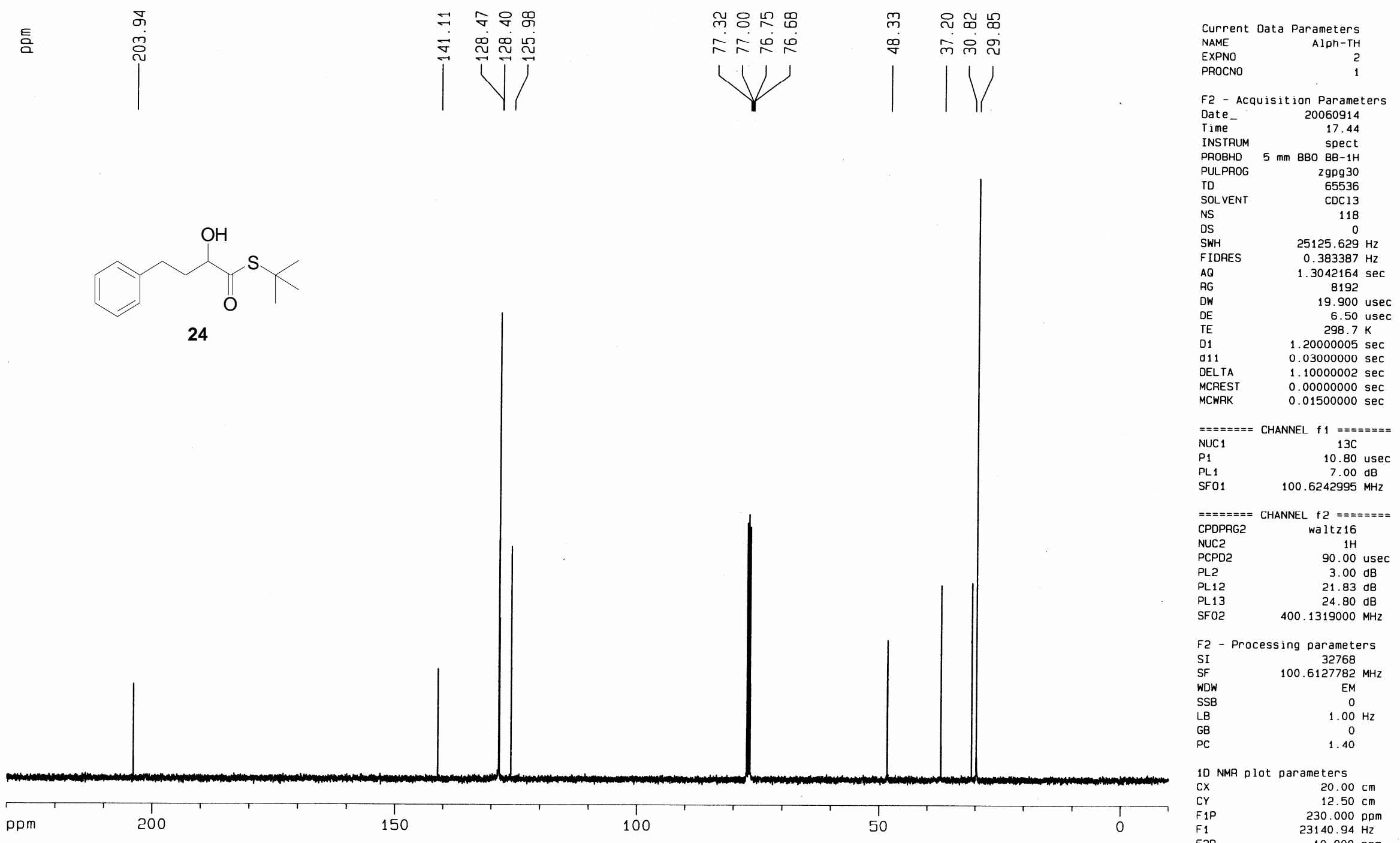

10 NMR plot parameters

$\begin{array}{ll}\text { CX } & \\ \mathrm{CY} & 20.00 \mathrm{~cm} \\ \mathrm{Fy} & 12.50 \mathrm{~cm}\end{array}$

$\begin{array}{lr}\text { F1P } & 230.000 \mathrm{ppm} \\ \text { F1 } & 23140.94 \mathrm{~Hz}\end{array}$

$\begin{array}{ll}\text { F2P } & -10.000 \mathrm{ppm} \\ \text { F2 } & -1006.13 \mathrm{~Hz}\end{array}$ $\begin{array}{lr}\text { F2 } & -1006.13 \mathrm{~Hz} \\ \text { PPMCM } & 12.00000 \mathrm{ppm} / \mathrm{cm} \\ \text { HZCM } & 1207.35327 \mathrm{~Hz} / \mathrm{cm}\end{array}$ 
$H$ spectrum of
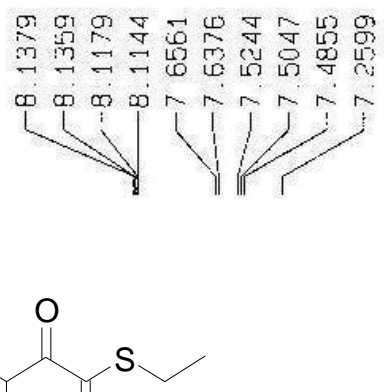

O

8a'
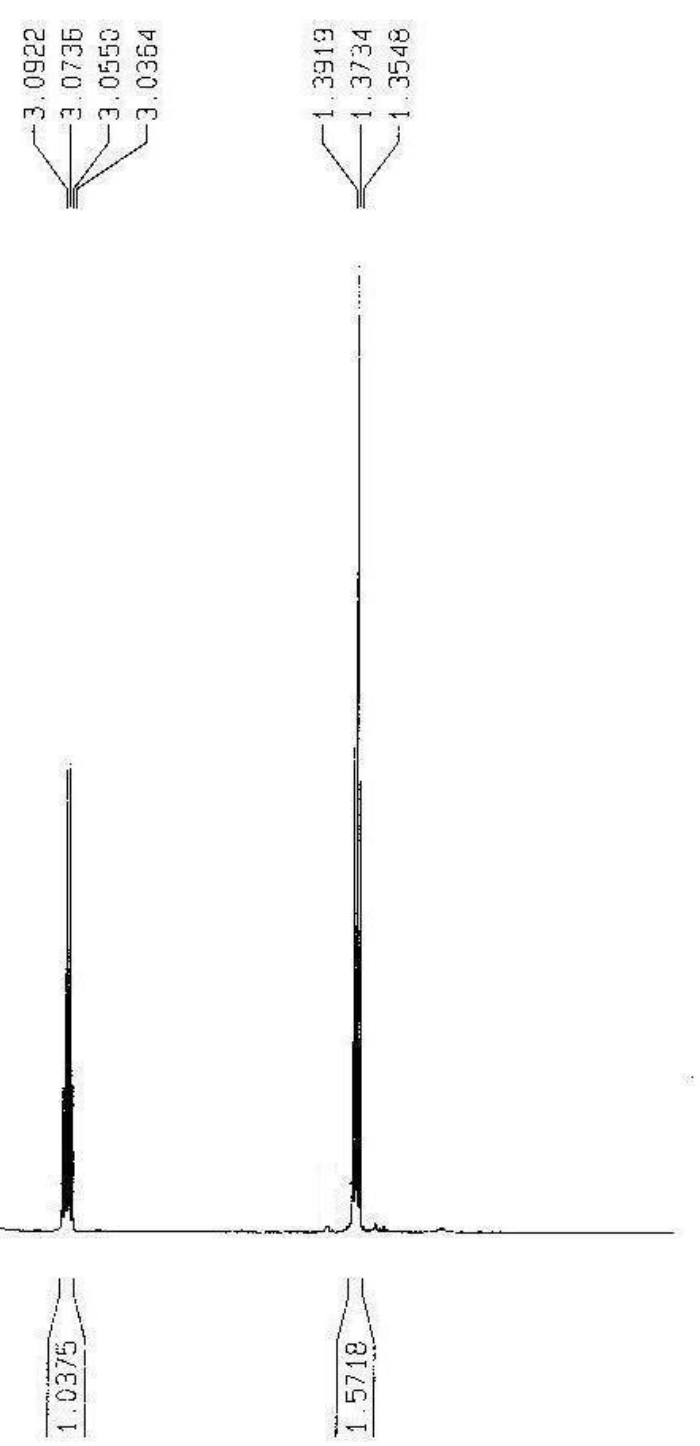

Current Data Paraneters ethane zn: $\mathrm{ket}$ $\begin{array}{ll}\text { EXPNO } & 1 \\ \text { PRDCNO } & 1\end{array}$

F2 - Acquisitjon Parameters

Date

Time 20.09

INSTRUM spert

TRCBHD 5 snim BBO BQ-1:

2930
जII PPOG

SOL.VFNT CDC13

$\begin{array}{ll}35 & 0 \\ 5 \text { WH } & 5995.204 \mathrm{~Hz}\end{array}$

IORES $\quad 0.35591 \mathrm{~A} \mathrm{HZ}$

AG $\quad 1.3664756 \mathrm{sec}$

7G 4054

JW $\quad 23.400$ usec

IE $300.0 \mathrm{~K}$

$31 \quad 1.50000000$

$$
\begin{aligned}
& ===-==- \text { CHANNEL } f 1===----- \\
& \text { NUC1 } 1 \mathrm{H} \\
& \text { ग.1 } 3.00 \mathrm{~dB} \\
& \text { SFD1 400.1326000 MH' }
\end{aligned}
$$

F2 - Processing parameters

$5 I \quad 16384$

SF $\quad 400.1300095 \mathrm{MHz}$

WDW EM

$\begin{array}{cc}55 B & \hat{0} \\ B & 0.10 \mathrm{~Hz}\end{array}$

$\begin{array}{lr}\mathrm{BB} & 0 \\ \mathrm{x} & 1.00\end{array}$

0 NMR plot oarameters

c* $\quad 20.00 \mathrm{~cm}$

CY $\quad 10.50 \mathrm{~cm}$

$-1 \mathrm{P} \quad \$ 0.500 \mathrm{ppm}$

1 $4201.37 \mathrm{~Hz}$

- 5 P -0.500 DPm

- $2000.37 \mathrm{~Hz}$

$\begin{array}{lr} & 0.55360 \mathrm{ppm} / \mathrm{cm} \\ \text { i. } 7 \mathrm{CM} & 220.07152 \mathrm{~Hz} / \mathrm{cm}\end{array}$

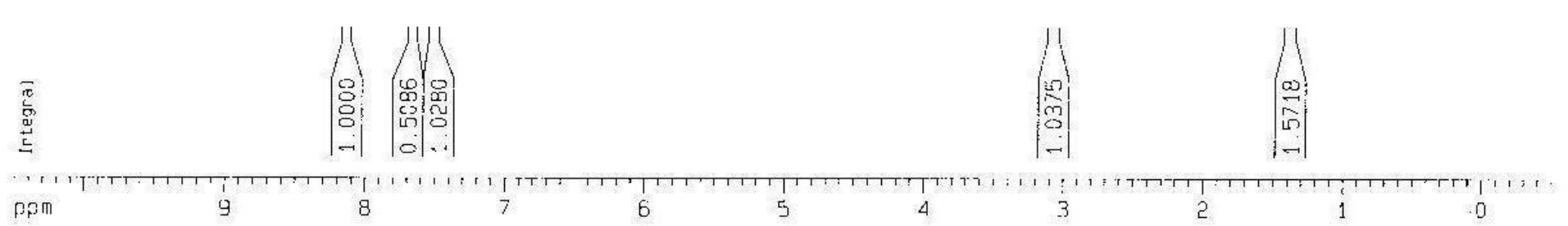




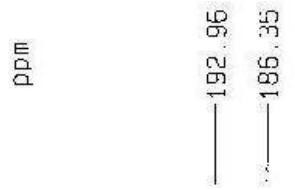

으요요요

감 $\vec{m}$ 윰

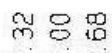

$\therefore N$ in

ฐָ

$\sqrt[1]{1}$

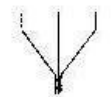

m

Current Dara Paramerers

FAME

PHOCNO

$=2$ - Acquisition Parameterrs

Jate_-

5 ตा вBO BQ-1H

$\begin{array}{ll}\text { PULPROG } & \text { 2909.30 } \\ \text { ID } & 65536\end{array}$

65.536
SOLVENT

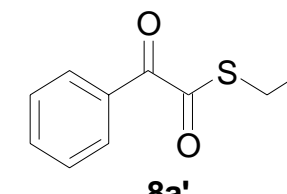

$8 a^{\prime}$

5WH $\quad 25 \div 25.629 \mathrm{HZ}^{\circ}$

$10 \mathrm{OHES} \quad 0.383387 \mathrm{~Hz}$

$86 \quad 1.3042164$ SE

$\begin{array}{lr}\text { DW } & 19.900 \text { usec } \\ \text { DE } & 6.50 \text { usec }\end{array}$

$6.50 \mathrm{USEC}$
$300.0 \mathrm{~K}$

$20000005 \mathrm{sec}$

$0.03000000 \mathrm{sec}$
$0.00002000 \mathrm{sec}$

$==-1, n=$ CHANNEL $11======$
NiC 1
$13 \mathrm{C}$

$\begin{array}{ll}\text { NuC } 1 & 13 \mathrm{C} \\ P_{1} & 10.80 \mathrm{usec}\end{array}$

PL1 $\quad 7.00 \mathrm{~dB}$

SFo

$=== \pm=$, CHANNEL $+2======$

CPDPRG2 walt 215

गCPD? 90.00 use

$\begin{array}{ll}\mathrm{LL} & 3.00 \mathrm{~dB}\end{array}$

$\begin{array}{ll}21.83 \mathrm{~dB} \\ \mathrm{~J}_{\mathrm{L} 13} & 24.80 \mathrm{~dB}\end{array}$

ग्य $4324.80 \mathrm{~dB}$

F2 - Processing parameters

$\begin{array}{lc}\text { SI } & 32768 \\ \text { SF } & 100.6127744 \mathrm{MHZ}\end{array}$

WOW $E M$

$\begin{array}{lc}S S B & 0 \\ -B & 1.00 \mathrm{~Hz}\end{array}$

学年

10 NMA plot parameters

zx $\quad 20.00 \mathrm{~cm}$

$\begin{array}{lr}\mathrm{Cr} & 7.00 \mathrm{~cm} \\ \mathrm{~F}=1 \mathrm{~m} & 220.000 \mathrm{pDm}\end{array}$

$\begin{array}{ll}=1.9 & 220.000 \mathrm{pDm} \\ =1 & 22134.81 \mathrm{~Hz}\end{array}$

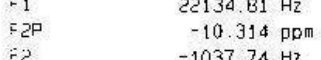

TPMCH $\quad-1037.74 \mathrm{~Hz}$

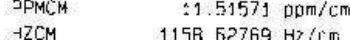


4 spectrum of

VAME
EXPNO
PROCNO

F2. Acquisitaon Parameters $\begin{array}{lr}\text { Jate_ } & 20060528 \\ \text { Ylme } & 16.23\end{array}$

YIm.e

INSTRUM 5 pect

PHOBHD $5 \mathrm{~mm}$ BBO BE-1.

PULPROG 2930

$\begin{array}{ll}\text { ID } & 16384 \\ \text { 50. NENT } & \text { CDCIJ }\end{array}$

ve

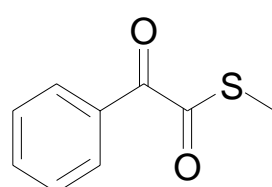

r

8b'

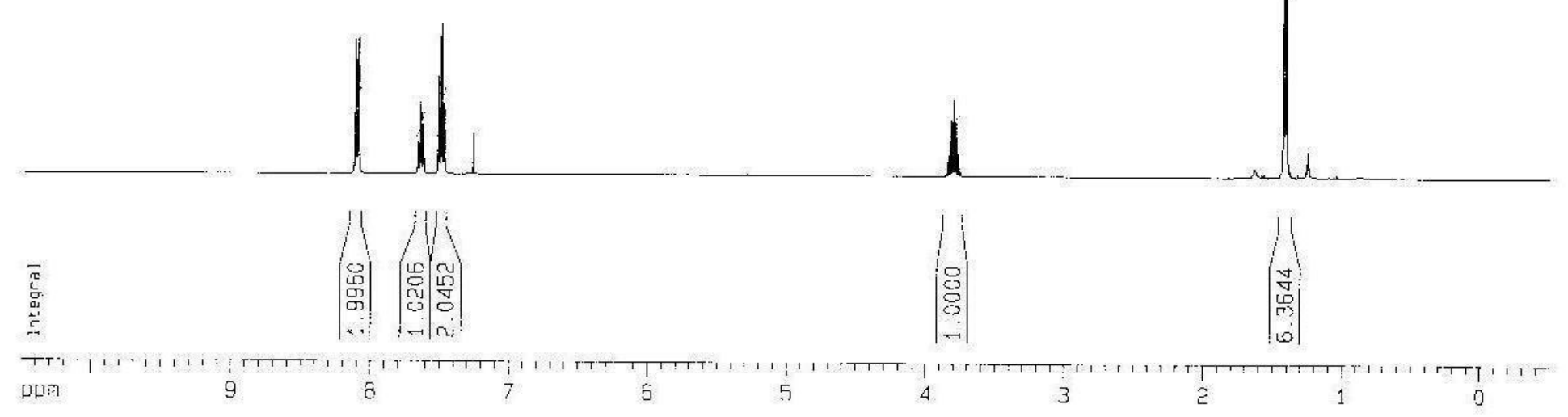

IS 16

SWH $\quad 5995.204 \mathrm{~Hz}$

Thes $\quad 0.365918 \mathrm{~Hz}$ 1. 3564756 sec

93. 414

6.50 บ5er 5.50 us $1.50000000 \mathrm{sec}$

$===-+=-$ CHANANFI $F 1==-===$ $\begin{array}{ll}\text { AUC1 } & 1 \mathrm{H} \\ \mathrm{P}_{1} & 10.10 \mathrm{u}\end{array}$ Pl. 1 3.00 $\mathrm{d}$ 용 sfor 400.

$=2$ - Processing paiameters SI 16384 SF $\quad 400.1300091 \mathrm{MI}$ SSB

$! B$
$G 6$
30

$$
\begin{gathered}
\mathrm{EM} \\
\mathrm{C} \\
0.15 \mathrm{~Hz} \\
0 \\
1.00
\end{gathered}
$$

10 NMA p]ct parameters

(0)

ᄃgp $\quad 10.000 \mathrm{Dpm}$

$=2 \quad 420.37 \mathrm{~Hz}$

$=2 \quad 0.500 \mathrm{pPm}$

JPMCM $\quad 0.55000 \mathrm{~Hz}$

$\mathrm{HZCM} \quad 220.07152 \mathrm{HF} / \mathrm{Cm}$
$20.00 \mathrm{~cm}$ 

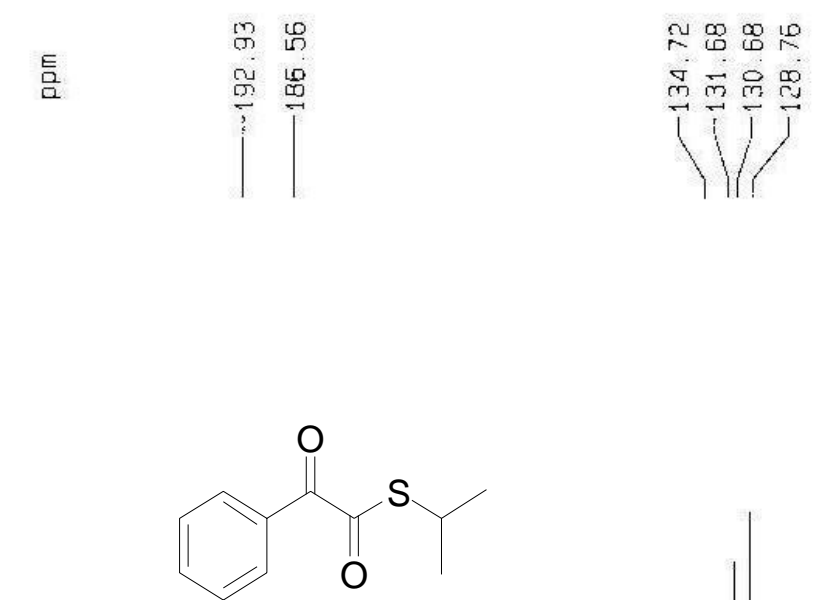

$8 b$

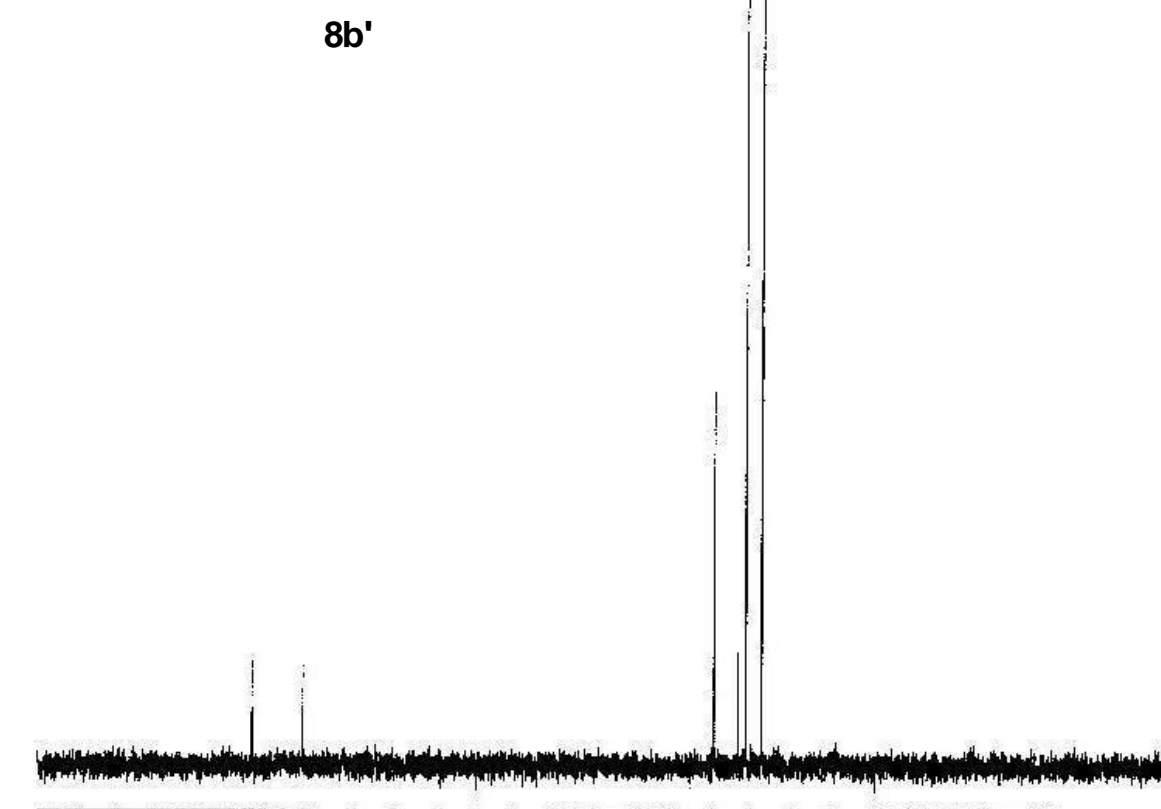

Eurrent Jata Parameters EXPNO Sati-TSO-HT-Ket. =xNo

F2 - A.cqu1situgn Parameters Date TIme

INSTRUM spect 5 MAl BBO $\mathrm{AB}-1 \mathrm{H}$ PIDOg 30 SOLVENT COCJ3 NS

SWH- $\quad 25125.629 \mathrm{~Hz}$ FIORES $\quad 0.393387 \mathrm{~Hz}$ $\begin{array}{ll}A Q & 1.3042154 \mathrm{sec}\end{array}$ JW $\quad 19.900$ usec Tt $300.0 \mathrm{~K}$ $31 \quad 1.20000005 \mathrm{sec}$ d11 $\quad 0.0300000058$ d12 $0.00002000 \mathrm{sec}$ $======$ CHANNEL $11======$
NUC1 $\begin{array}{ll}\text { MUC1 } & 13 C \\ 1 & 10.80,45\end{array}$ $\begin{array}{lr}\text { DL1 } & 7.00 \mathrm{AB} \\ \text { SFO1 } & 100.6242995 \mathrm{MHz}\end{array}$ $======-6$ HANNEL $f 2======-$ 5PLPAS5 waltz ti $\begin{array}{ll}\text { NUC2 } & 111 \\ \text { जCPL2 } & 90.00 \text { use }\end{array}$ FL2 $3.00 \mathrm{de}$ FL12 21. 93 d4

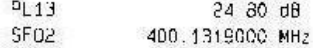

$=2$ - Processing pararreteres $=21$ - Processing parameter's
SI 32768 . jif 100. 1127729 MH? WSOW

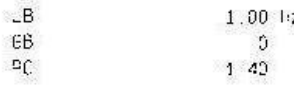

10 NNR? plot parameters

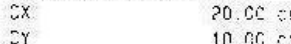

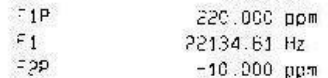
$\begin{array}{ll}=2 & -10.000 \mathrm{np} \\ =2 & -1006.13 \mathrm{~Hz}\end{array}$ PPMCM 11.50000 pomill:m 
$H$ spectrum of

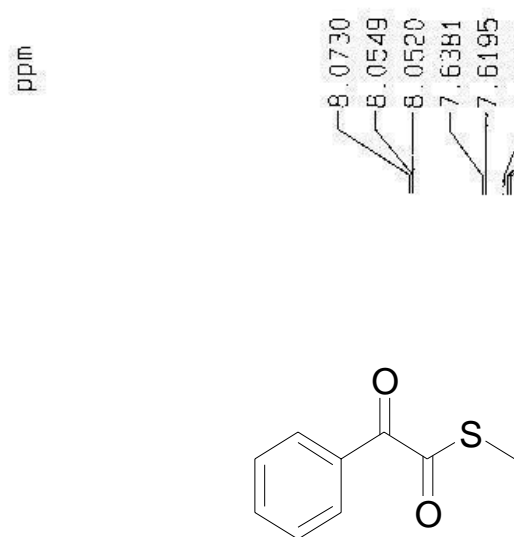

$8 c^{\prime}$

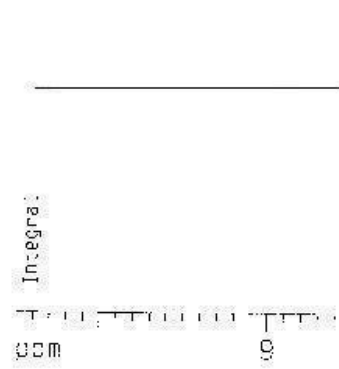

Lid.

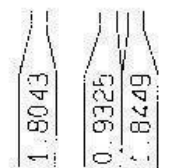

$-T: 1$
Uอm?

e

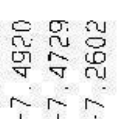

;

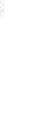

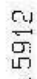
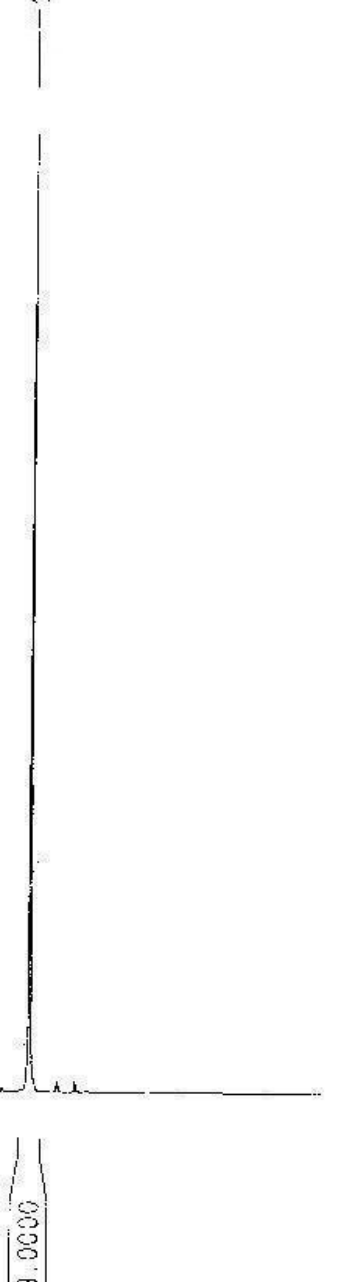

Curnent bata Рa:areters

vaME Sam-EhEnY]-HT-KI-H

OXPNO

a - Accuisition farameters

date. 2006052B

Tamé

JNETRIM spert

P.LPGO

IDLPFOE 2930

SOLVENT

is

$\begin{array}{ll}\text { JE } & 0 \\ \text { SWH } & 5995.204 \mathrm{~Hz} \\ \text { FIDFES } & 0365918 \mathrm{~Hz}\end{array}$

IDFES O $365918 \mathrm{~Hz}$

1. 365475E SEC

TE

3

63 1140 user

5. 50 usec $3000 \mathrm{~K}$

$== \pm=-=$ SHANNEL

NUC1

$D_{1} \quad 10.1 \mathrm{H}^{10}$

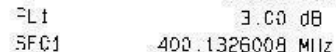

22 - Processing parameters

SI 16384

Wit $400: 300091$ MAll

WDW $\quad: M$

$\begin{array}{ll}S 5 B & 0 \\ \therefore B & 0.10 \mathrm{~Hz}\end{array}$

$\therefore \mathrm{C}$

15 NWI fiot farameters

cx $20.00 \mathrm{~cm}$

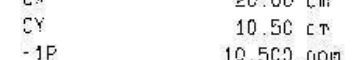

$\begin{array}{ll}-1 F & 10.509 \\ \because-3017 & 420137 \mathrm{~Hz}\end{array}$

$\begin{array}{lr}\because & 420137 \mathrm{~Hz} \\ =\hat{Z} \mathrm{P} & 0500 \mathrm{DDm}\end{array}$

$=2 \quad-200.07 \mathrm{~Hz}$

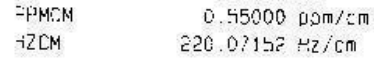




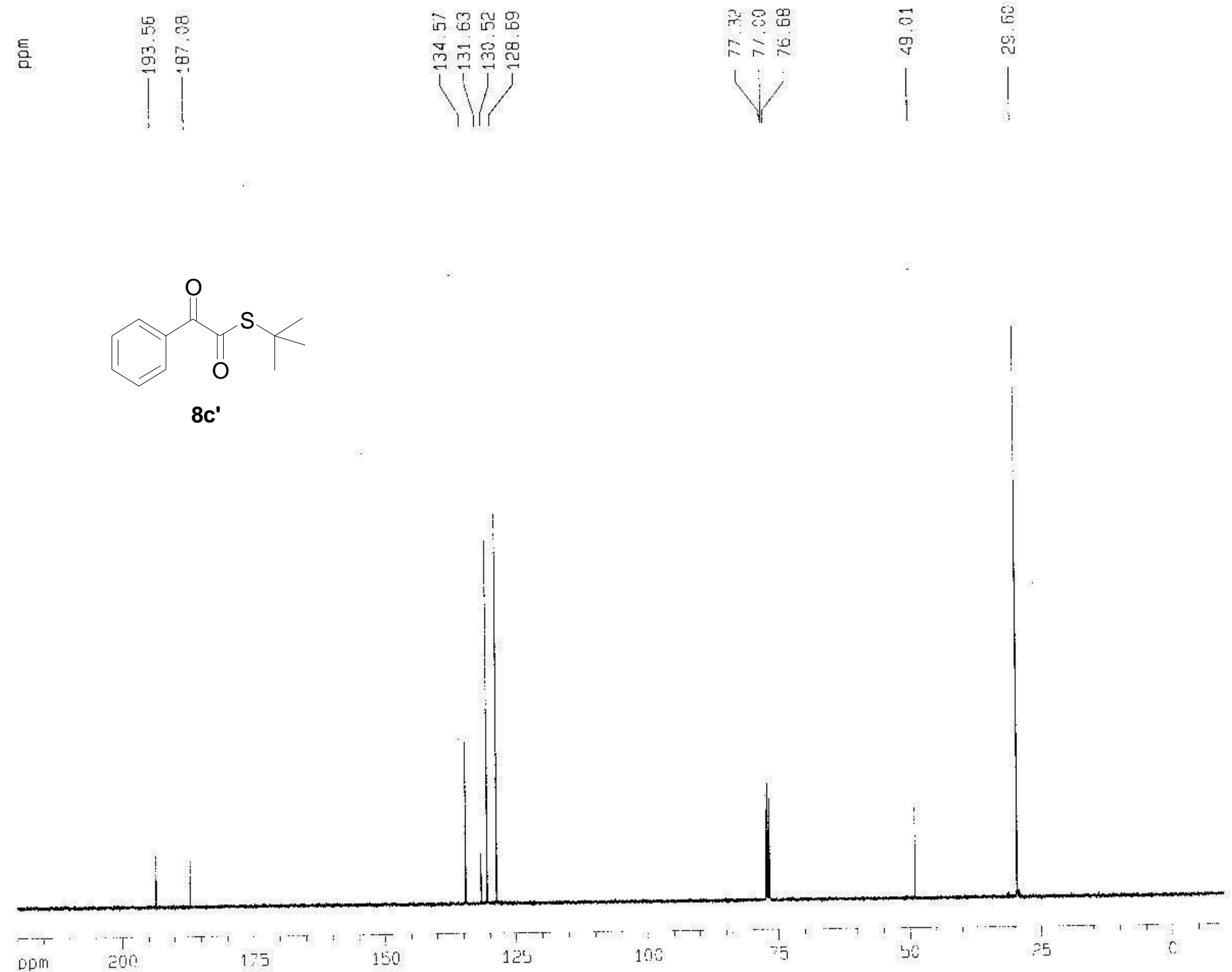

Gur. enent Dütō Farameters

YAME

STOC NOD

terthis

$=2-4 c a d$ isitjon Farameler

בaic. 2005101

Tिए $12.3 \%$

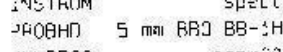

LLPROE 2 FF.930

ร. YENT

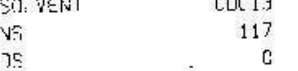

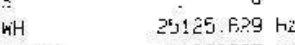

=UHES $\quad 0.3 \mathrm{AB} 3337 \mathrm{HC}$

Lis 1.3042154 ser.

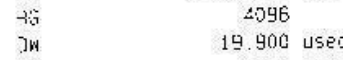

$\mathrm{JE} \quad$ f. 50 บ5:

120000005

11: $\quad 0.03000000 \mathrm{ERC}$

$12 \quad 0.00002000$ se:

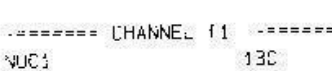

10.80 use.

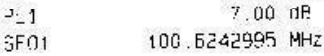

$=====-$. CHANNEL $+\hat{2}== \pm== \pm$

CPEPAG? Wâ?

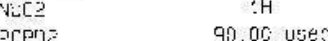

पr 30 and

PL12 21 $1.93 \mathrm{di3}$

L:3 खो

SFO2 450 1:11 $1000 \mathrm{C}$ Mा:?

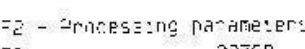

L1 350

5i $\quad 1511.61>7775$ M MHZ

- 358

Sh

1:. NHA plet carzmeter's

. $200 \mathrm{zm}$

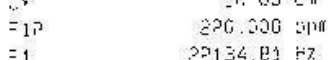

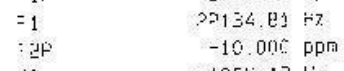

-toot $13 \mathrm{lic}_{\mathrm{i}}$

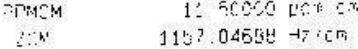


$H$ speztrum 0 !

言
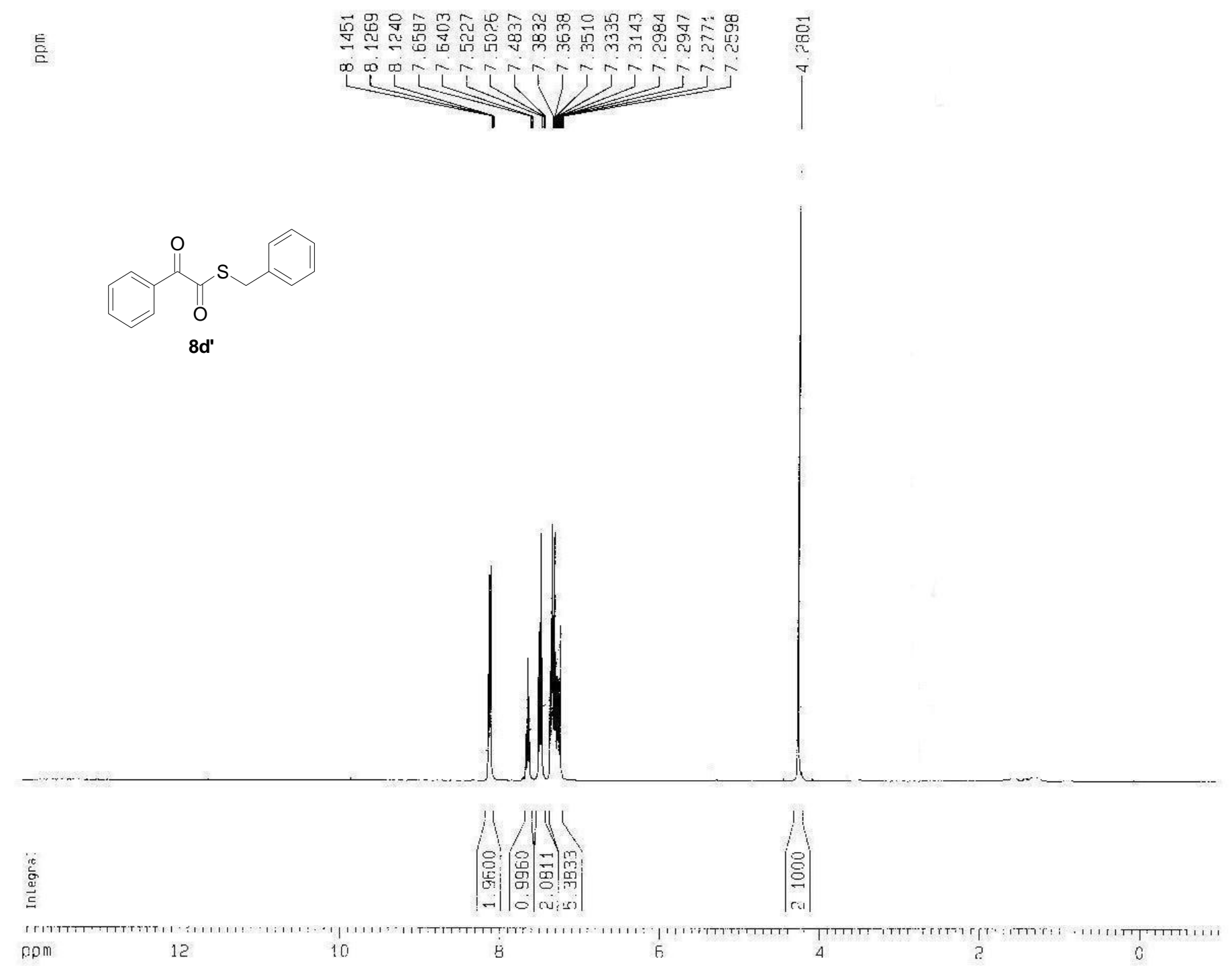

EXPND

zz - Acquisition Parameters

Jate_ 20060528

T1lise

1415

INSTR.JM SIPELt

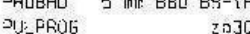

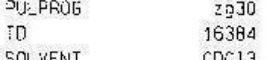

Sol

5hit

al] $\quad 1.3654755 \mathrm{~Hz}$

203.2

83.400 ušec
DF

$\begin{array}{ll}\text { TE } & 300.0 \mathrm{~K} \\ \text { D1 } & 1.50000000 \text { 5ec }\end{array}$

$x=m====$ CHANNEL $f 1$ = $======$

$\begin{array}{lll}\text { YULC1 } & 14 \\ y_{1} & 10.10 \text { บรec }\end{array}$

ग.1 $3.00 \mathrm{dg}$

SFE.1 $400.1326008 \mathrm{MHz}$

$=2$ Processsng parameters

$\begin{array}{lc}\text { SI } & 16384 \\ \text { SF } & 400.1300095 \mathrm{MHz}\end{array}$

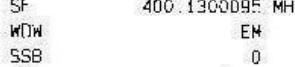

SSB
LB

5e

17. NMA plot parameters

$\begin{array}{ll}z X & 20.00 \mathrm{~cm} \\ z y & 10.50 \mathrm{~cm}\end{array}$

$1 \mathrm{P} \quad 13.968 \mathrm{opm}$

$=2 \mathrm{H}-1 . \mathrm{H}$

-2 -4.6. $25 \mathrm{~Hz}$

$\begin{array}{ll} & 0.76916 \mathrm{pDm} / \mathrm{cm} \\ -7 \mathrm{CM} & 299.76019 \mathrm{~Hz} / \mathrm{cm}\end{array}$ 
筮 8

$\therefore=10$

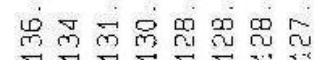
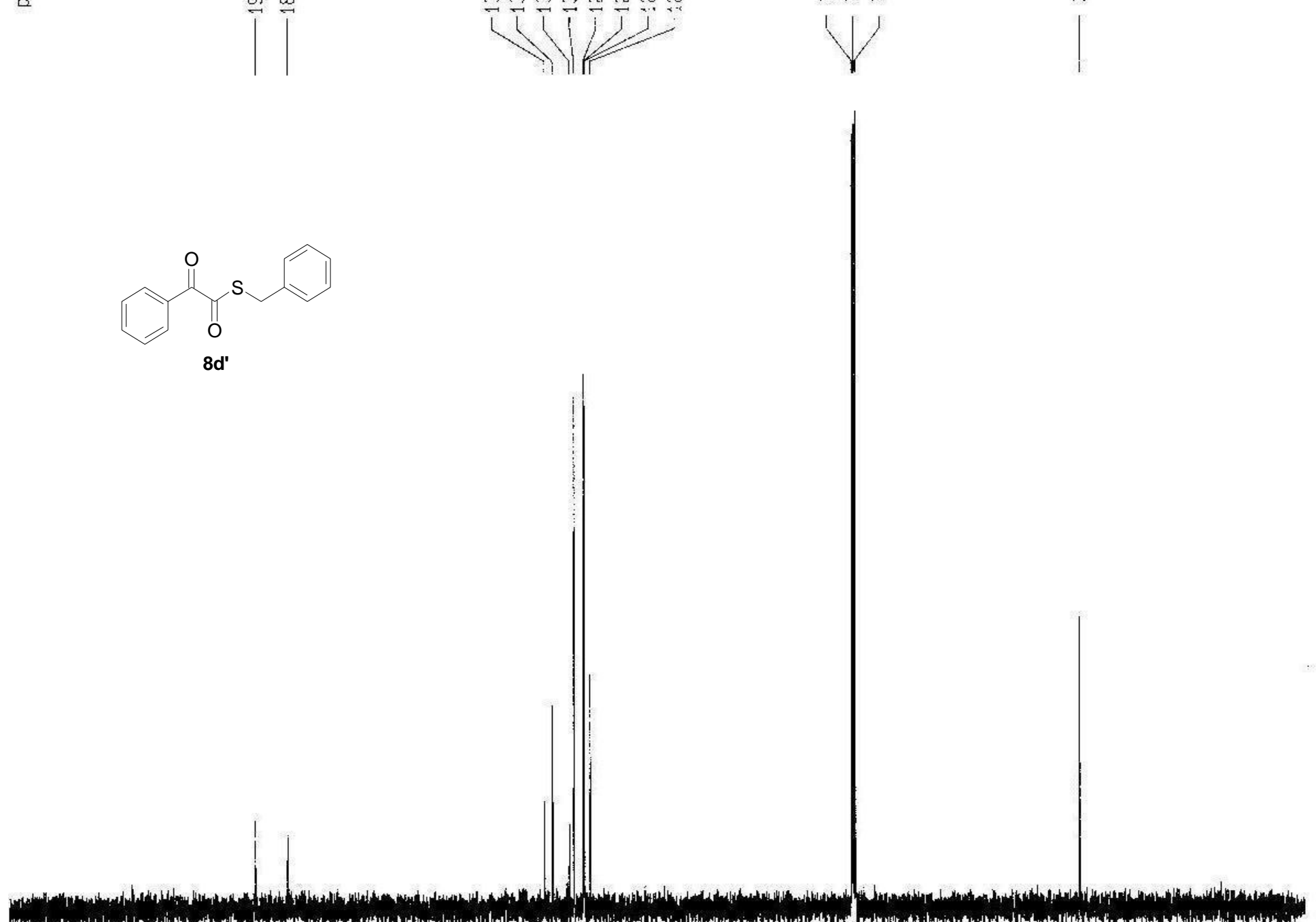

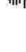

200
175
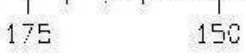

150

125

100

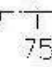

50
Current Dita Parametera MAME Sam -S-benzy l-Ket-H EXPND

ROCN i

F2 - Arzuisition Parameters

rims

spect

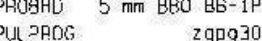

T0

SOL VENT COE13

NS

SWH $25125.625 \mathrm{~Hz}$

IUPES $\quad 0.38338 \% \mathrm{~Hz}$

1.3042164 se

RG 4096

$\begin{array}{rr}19.900 \text { usEe } \\ \text { DE } & 6.50 \text { user }\end{array}$

$300.0 \mathrm{~K}$

$011 \quad-\quad 0.03000000 \mathrm{sec}$

$112 \quad 0.00002000$ sec

$== \pm===$ CHAMNEL 11 $== \pm===$

WuC: $13 \mathrm{C}$

$s_{1} \quad 10.80$ usec

$\begin{array}{lr}\text { ग1 } & 7.00 \mathrm{~dB} \\ \text { SFE1 } & 100.6242995 \mathrm{MHz}\end{array}$

-... CHANNEL $f$ ? $=======$

CPCPAGe waltz16

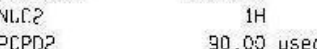

$\begin{array}{ll}1 \% & 3.00 \mathrm{de}\end{array}$

$21.83 \mathrm{de}$
$24.80 \mathrm{de}$

SFO2 $400.1319000 \mathrm{MH}+\mathrm{Z}$

ic - Processing parameters

5 a $3275 \mathrm{~B}$

WDYit $\quad 100.612772 ! M$

$\begin{array}{lc}\text { SSE } & 0 \\ \mathrm{BB} & 1.00 \mathrm{~Hz}\end{array}$

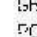

10) AMAR 0:10t piaramieters

Cx $\quad 20.00 \mathrm{~cm}$

$\begin{array}{lr}c y & 1250=\mathrm{cm} \\ =13 & 399.435 \mathrm{oDir}\end{array}$

$=1 \quad 24090.19 \mathrm{~Hz}$

$\begin{array}{ll}-1 & 10291 \mathrm{prm} \\ 2-2 & -10.35 .14 \mathrm{H7}\end{array}$

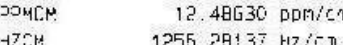




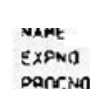

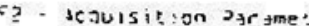

Dote- $\quad 2006.519$

ine

1953

Nopiat soect

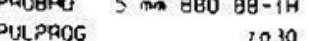

io

SOL VEN

w5

OS

15

5995.204

TORES 0.365918 :

A0 $\quad 1.3604756$ s

7622.5

OE 33.400
TE
6.50

TE:

$1.50000000 \mathrm{~s}$

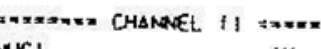

NuC1

Pi

F2 - Processing oar ameter

$51 \quad 16384$

400.1300095

SSO

S58

co

$G B$

10 mo oloc oraneters

cy

F!P

F!

$82 p$
52

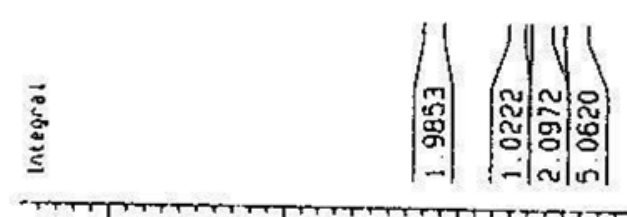

P्सC

HZCH

Ex

$0.10 \mathrm{H}$

0

meters

$9.00 \mathrm{c}$

10.500 o1

4201.37 H

-0.500 or

$-200.07 \mathrm{H}$

0.55000 or

220.07152 ㄴ 

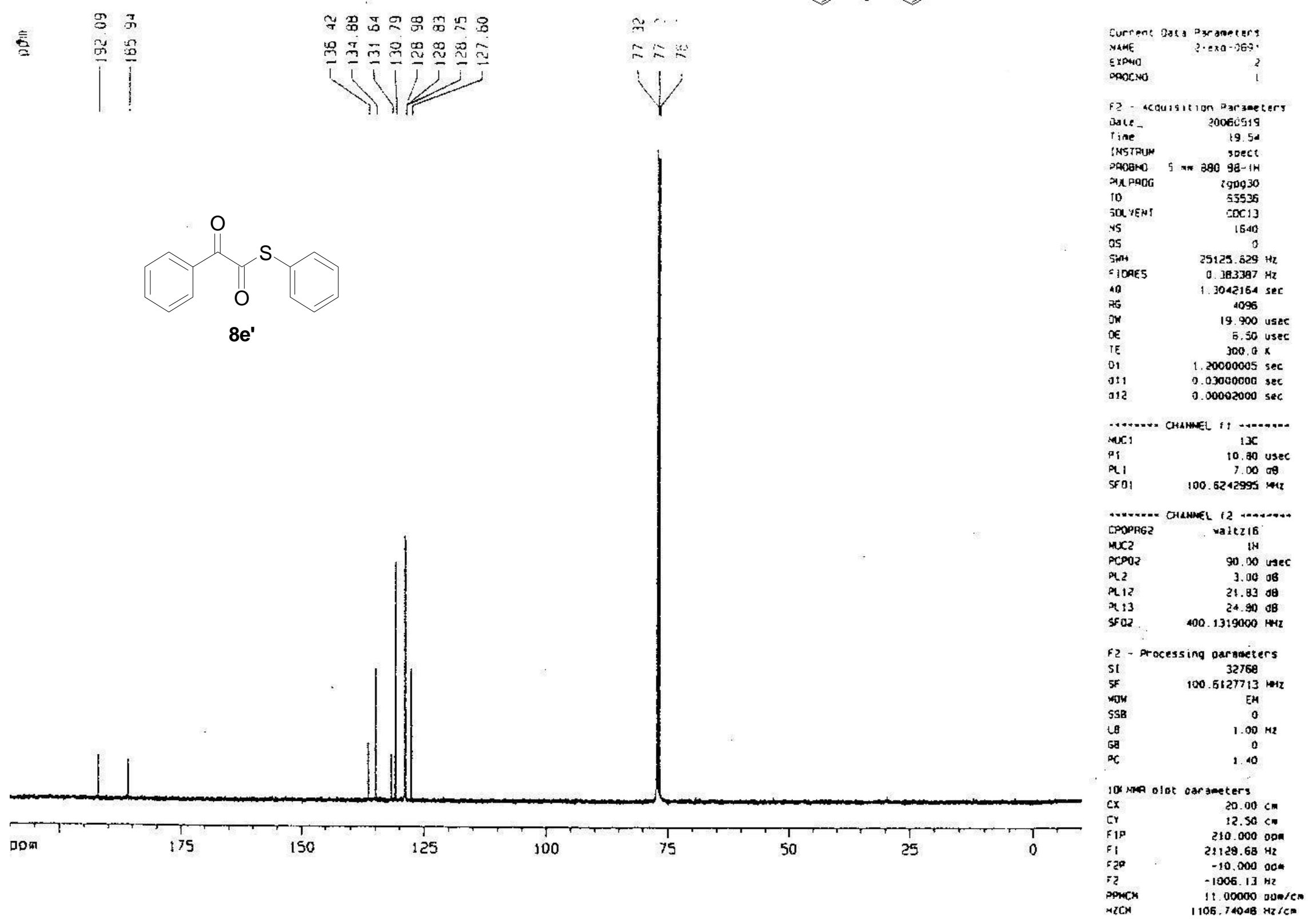
$H$ spectrum of

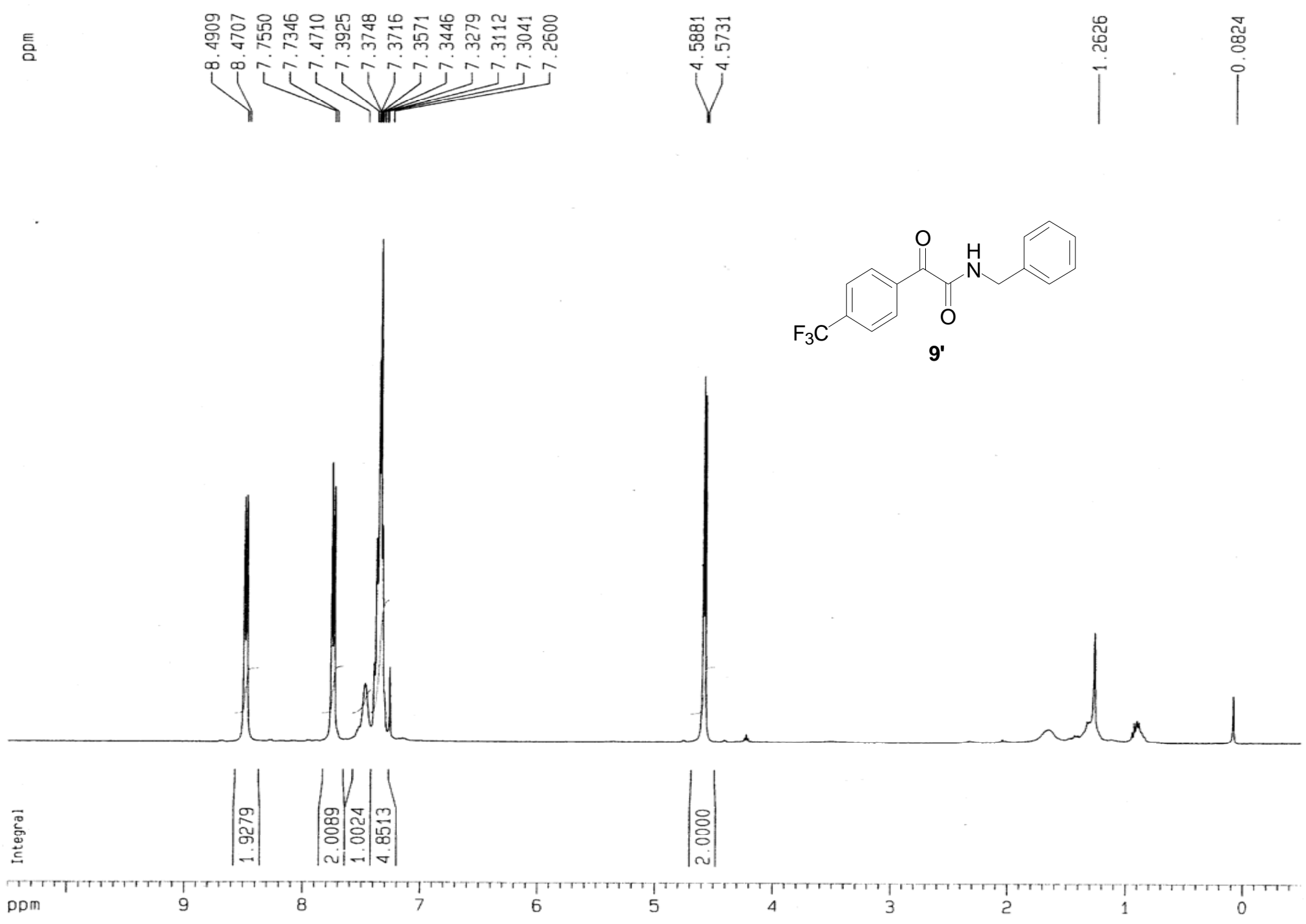

Current Data Parameters

NAME

EXPNO

CF3medalate

F2 - Acquisition Parameters

Date _ 20051213

Time

12.46

INSTRUM spect

PROBHD $5 \mathrm{~mm}$ BBO BB-1H $\begin{array}{lr}\text { PULPROG } & 2930 \\ \text { TD } & 16384\end{array}$

SOLVENT CDC13

SS 1 SW

FIDRES $\quad 0.365918 \mathrm{~Hz}$

$A Q \quad 1.3664756 \mathrm{sec}$

RG 181

$\begin{array}{lr}\text { DW } & 83.400 \text { usec } \\ \text { DE } & 6.50 \text { usec }\end{array}$

TE $300.0 \mathrm{~K}$

D1 $1.50000000 \mathrm{sec}$

NUC1 CHANNEL f1 $== \pm== \pm=0$

$P_{1} \quad 10.10$ use

SF01 $400.132500 \mathrm{~dB}$

F2 - Processing parameters

SI Processing 15384

$\begin{array}{lc}\text { SF } & 16384 \\ \text { SF } & 400.1300099 \mathrm{MHZ}\end{array}$

WDW $E M$

$\begin{array}{lc}\text { SSB } & 0 \\ \text { LB } & 0.10 \mathrm{~Hz} \\ \text { GB } & 0\end{array}$

$\begin{array}{lr}\text { GB } & 0 \\ P C & 1.00\end{array}$

10 NMR plot parameters

cX $\quad 20.00 \mathrm{~cm}$

$\begin{array}{ll}\mathrm{CY} & 9.00 \mathrm{~cm}\end{array}$

$\begin{array}{lr}10.500 \mathrm{DDm} \\ \mathrm{F} 1 & 4201.37 \mathrm{~Hz}\end{array}$

$\begin{array}{ll}F 1 & 4201.37 \mathrm{~Hz} \\ \text { F2P } & -0.500 \mathrm{ppm}\end{array}$

PPMCM $\quad-200.07 \mathrm{~Hz}$

$\mathrm{HZCM} \quad 220.07152 \mathrm{~Hz} / \mathrm{cm}$ 
C13 spectrum of

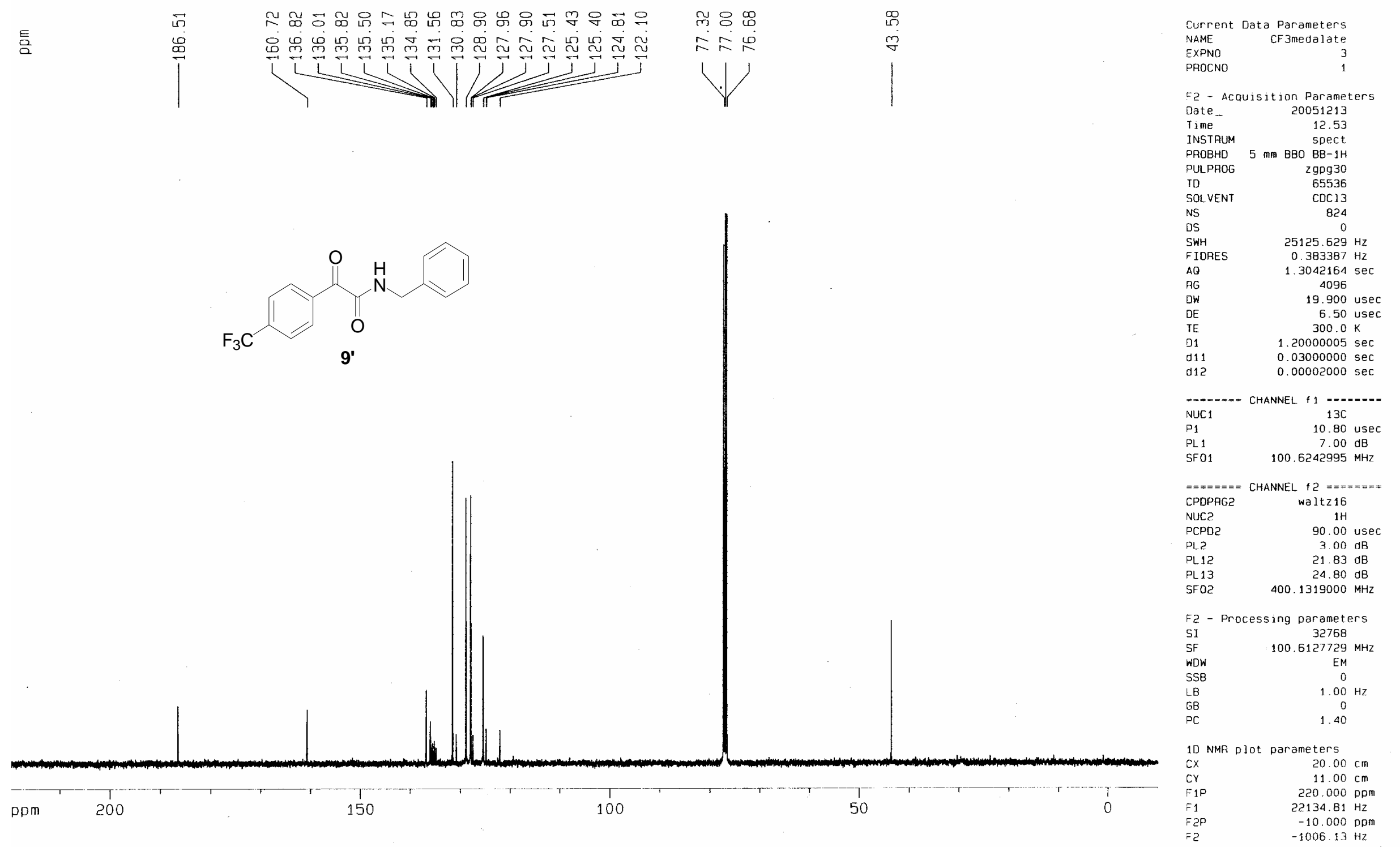


$\mathrm{H}$ spectrum of
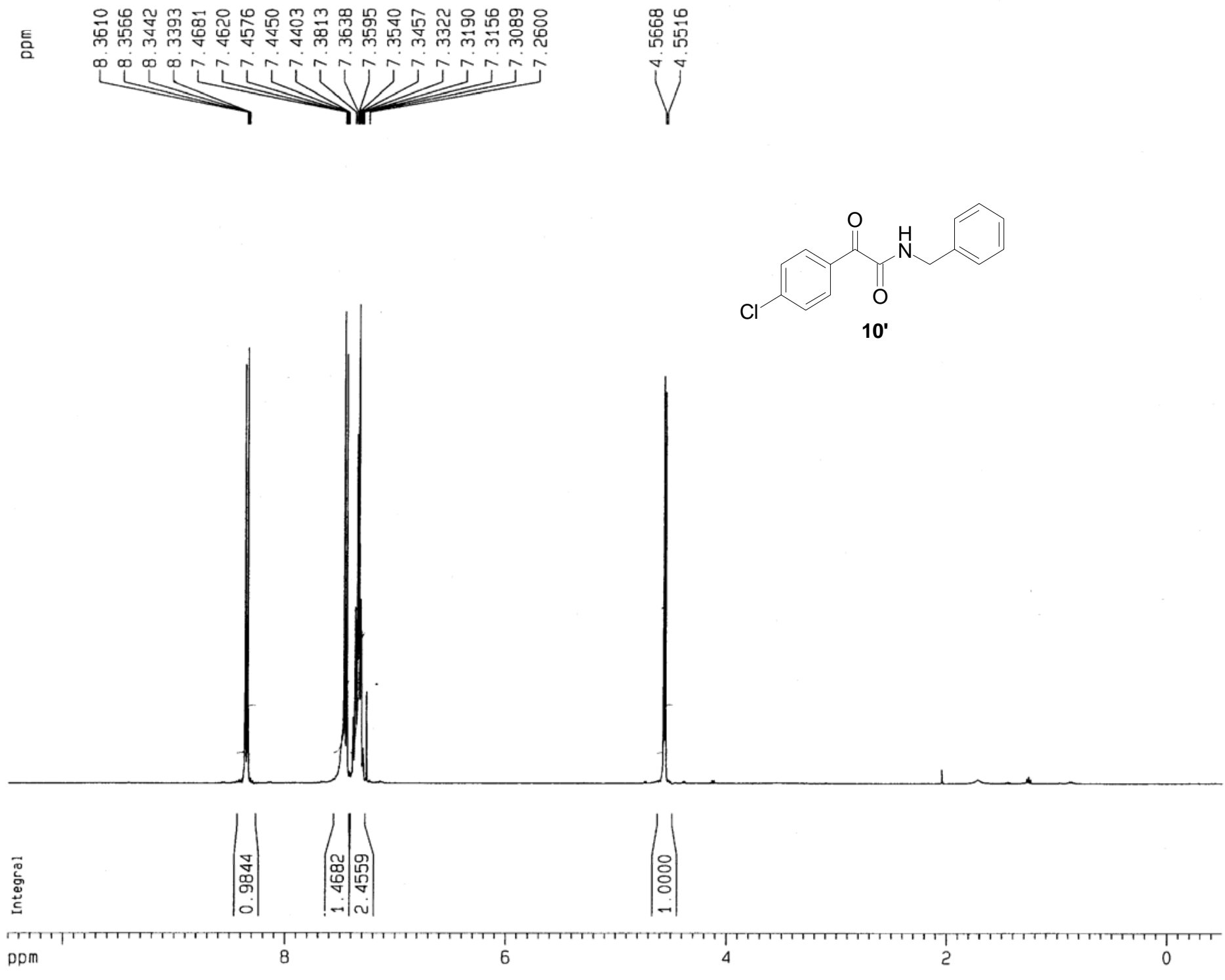

Current Data Parameters

EXPNO

3

F2 - Acquisition Parameters

Date_ 20050304

INSTRUM 16.48

PROBHO $5 \mathrm{~mm}$ BBO BB-1H

PULPROG 2930

$\begin{array}{ll}\text { TD } & 16384 \\ \text { SOLVENT } & \text { COC13 }\end{array}$

NS

SWH $\quad 5995.204 \mathrm{~Hz}$

FIDRES $\quad 0.365918 \mathrm{~Hz}$

AQ $\quad 1.3664756 \mathrm{sec}$

$\begin{array}{lr}\text { AG } & 22.6 \\ \text { DW } & 83.400 \text { usec }\end{array}$

DE $\quad 6.50$ use

$1.5000000 \mathrm{~K}$

$== \pm \equiv= \pm=$ CHANNEL $f 11=\equiv= \pm= \pm$

NUC1 $1 \mathrm{H}$

$\begin{array}{lr}\text { P1 } & 10.10 \text { use } \\ \text { PL1 } & 3.00 \mathrm{~dB}\end{array}$

SF01 $400.1326008 \mathrm{MHz}$

F2 - Processing parameters

SI 16384

SF $\quad 400.1300088 \mathrm{MHZ}$

WDW

SSB

GB $\quad 0.10 \mathrm{~T}$

10 NMR plot parameters

CX prot paranes

$\mathrm{CY}$

F1P $\quad 10.000 \mathrm{~cm}$

F1 $\quad 4201.37 \mathrm{~Hz}$

$\begin{array}{ll}F 2 P & -0.500 \mathrm{pDm} \\ \mathrm{F} 2 & -200.06 \mathrm{~Hz}\end{array}$

PPMCM $\quad 0.55000 \mathrm{ppm} / \mathrm{c}$

HZCM $220.07150 \mathrm{~Hz} / \mathrm{cm}$ 

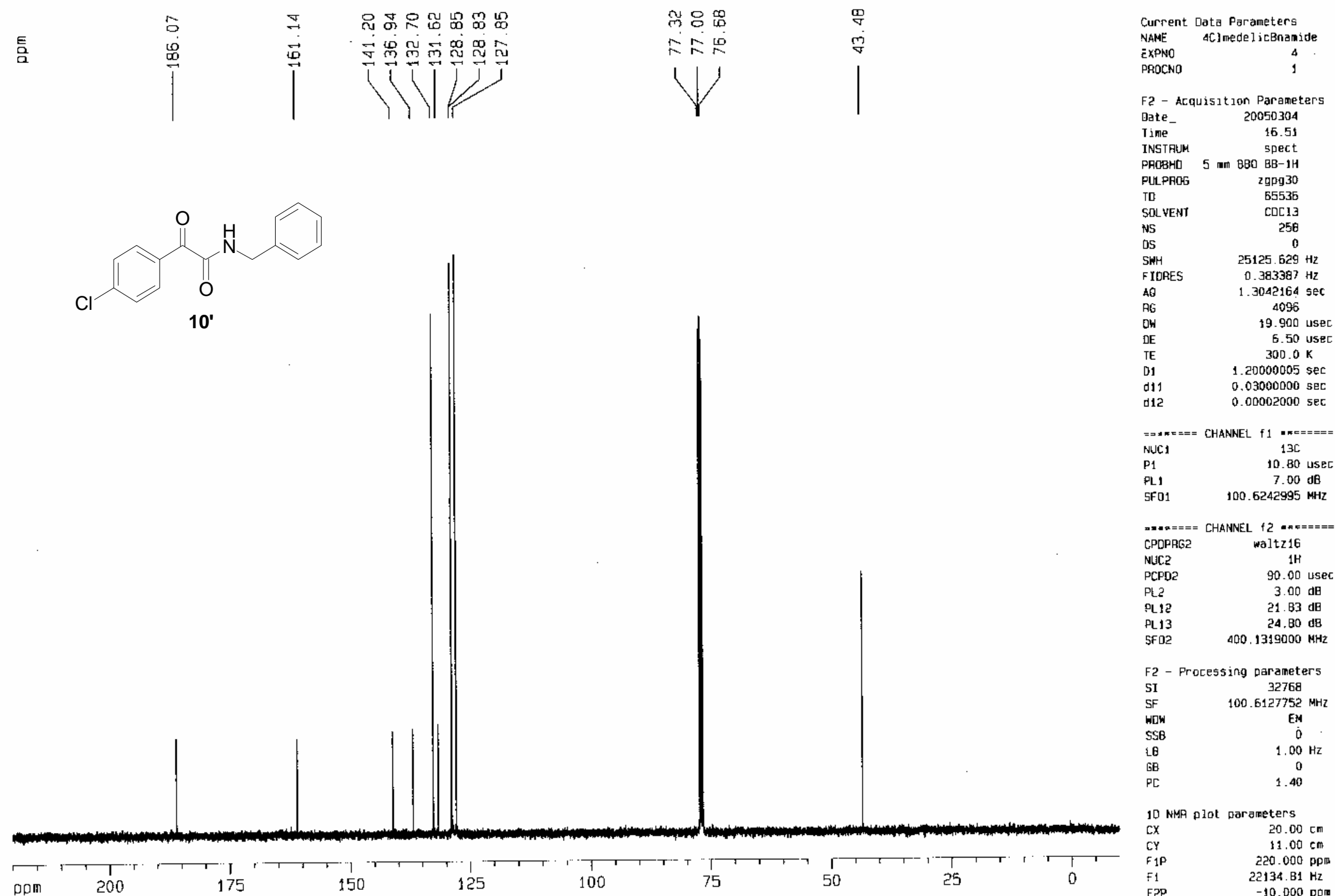

10 NMP plot parameters

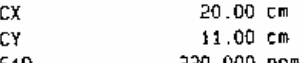
$220.000 \mathrm{ppm}$ $22134 . \mathrm{B1} \mathrm{Hz}$ $-10.000 \mathrm{pora}$ 
$H$ spectrum of
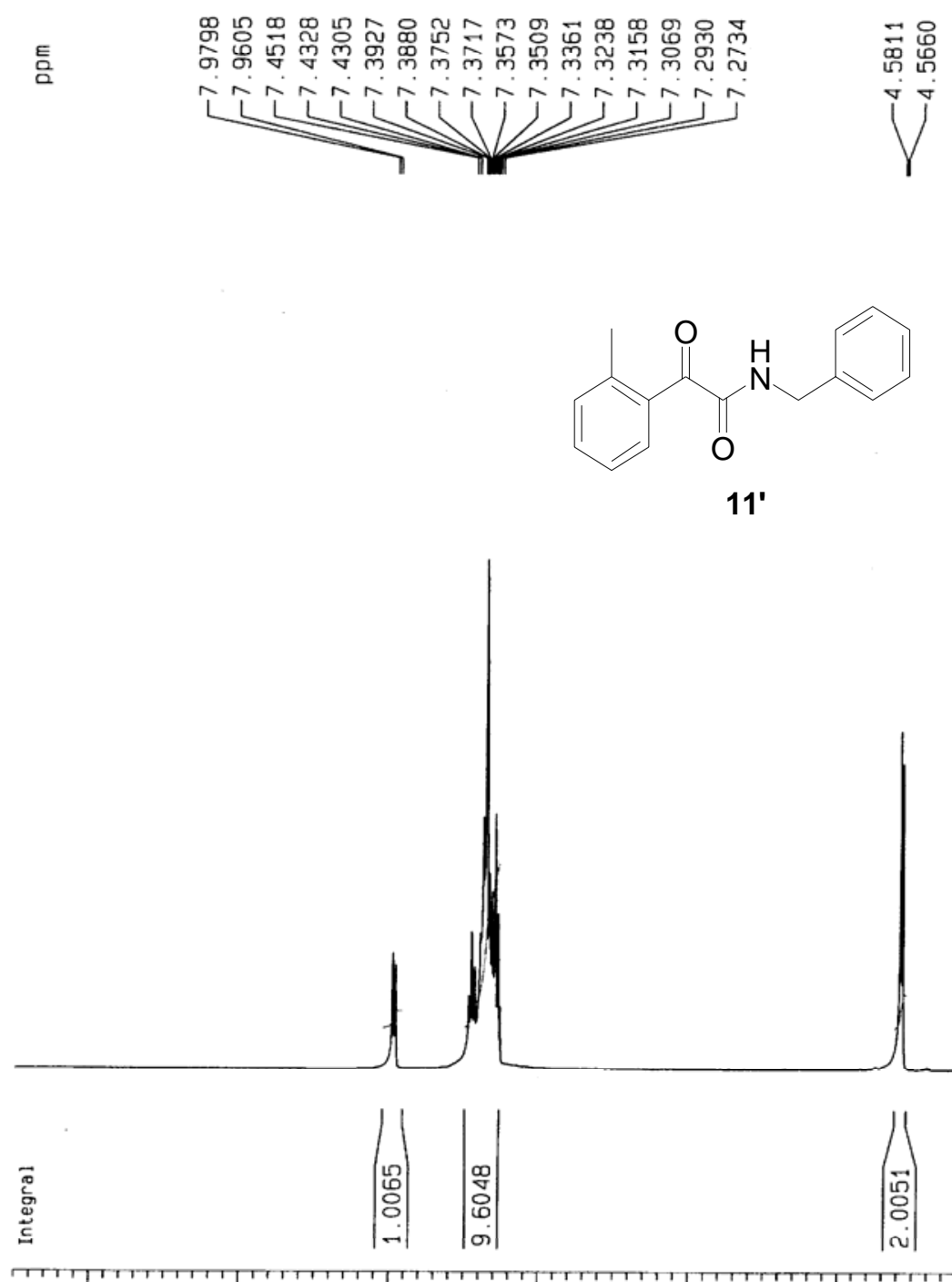

ppm

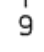

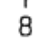

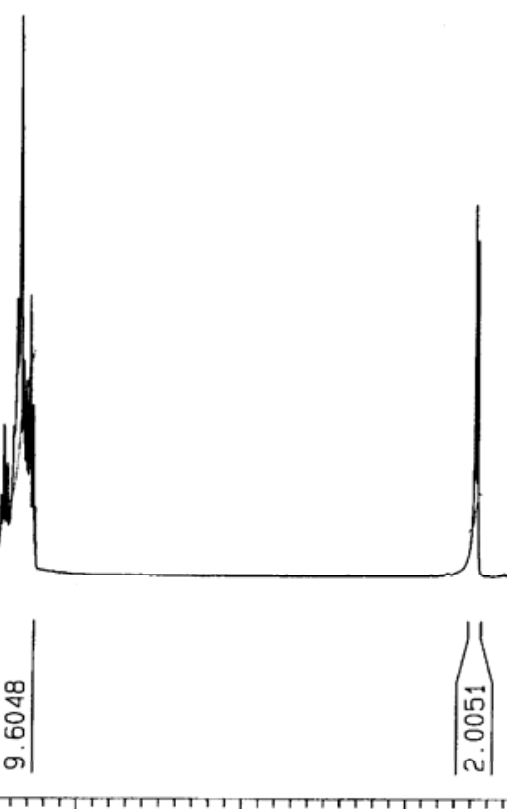

Current Data Parameters

NAME

PROCNO

F2 - Acquisition Parameters

Date _ 20050608

15.12
50.12

INSTRUM Spect

PROBHD $5 \mathrm{~mm}$ BBO BB-1H

PULPROG 2930

TD

SOLVNT

DS

SWH $\quad 5995.204 \mathrm{HZ}$

$\quad 0.365918 \mathrm{~Hz}$

$\begin{array}{lc}\text { AQ } & 1.3664756 \mathrm{sec} \\ \text { RG } & 128\end{array}$

DW $\quad 83.400$ usec

6.50 use

$1.50000000 \mathrm{sec}$ $==z=z= \pm$ CHANNEL $f 1== \pm=z=m$

$\begin{array}{ll}\text { NUC1 } & 1 \mathrm{H} \\ \mathrm{P} 1 & 10.10 \mathrm{use}\end{array}$

PL1 $3.00 \mathrm{~dB}$

SF01 $400.1326008 \mathrm{MHz}$

F2 - Processing parameters

SI 16384

SF $\quad 400.1300095 \mathrm{MHz}$

WOW

SSB

LB

$$
\begin{gathered}
\text { EM } \\
0 \\
0.10 \mathrm{~Hz} \\
0
\end{gathered}
$$

0
1.00

10 NMR plot parameters

CX $\quad 20.00 \mathrm{~cm}$

$\begin{array}{ll}\text { CY } & 10.50 \mathrm{~cm}\end{array}$

F1 $\quad 10.500 \mathrm{ppm}$

$\begin{array}{ll}F 1 & 4201.37 \mathrm{~Hz} \\ F 2 P & -0.500 \mathrm{pPm}\end{array}$

F2 $\quad-200.07 \mathrm{Hzm}$

$\begin{array}{lr}\text { PPMCM } & 0.55000 \mathrm{ppm} / \mathrm{cm} \\ \text { HZCM } & 220.07152 \mathrm{~Hz} / \mathrm{cm}\end{array}$

$\mathrm{HZCM}$

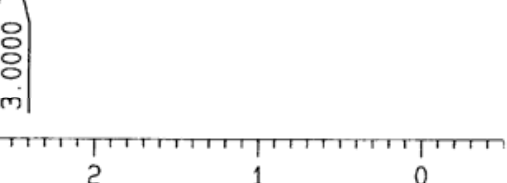


C13 spectrum of

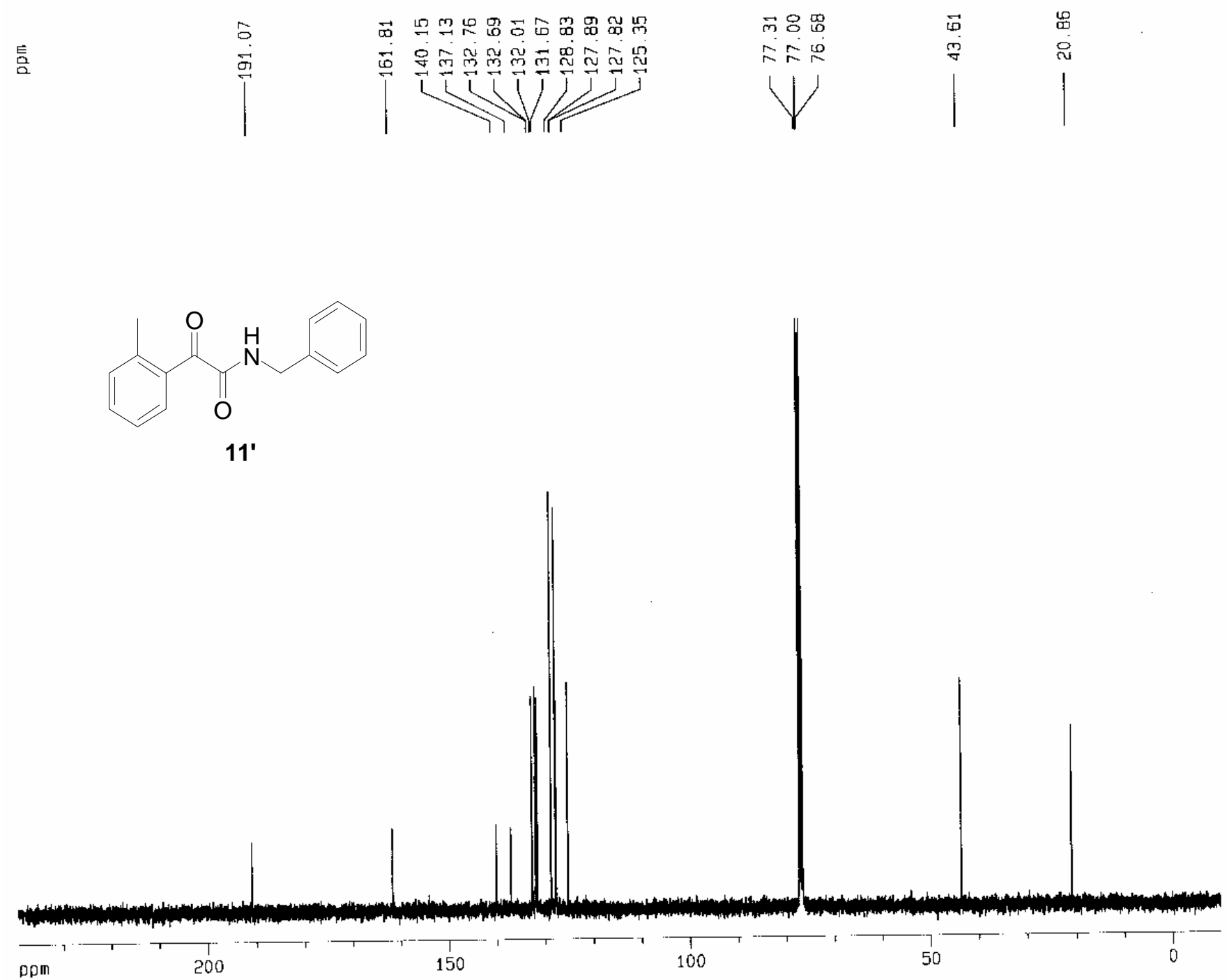

Current Dat a Paraneters

20050608-1

22- Acquisition Paraneters

Time

15.15

5 ตाr $860 \mathrm{BB}-1 \mathrm{H}$

PULPRDG $\quad 2$ ggg 30

SOLVENT $\quad$ CDC 13

$\begin{array}{lr}\text {.NS } & 329 \\ 05 & 0\end{array}$

5WH $25125.629 \mathrm{HZ}$

$1.3042164 \mathrm{sec}$

8000

19.900 uset

5. 50 usec

$\begin{array}{ll}\text { Te } & 1.20000005 \\ 01 & 5 \mathrm{sec}\end{array}$

$\begin{array}{ll}011 & 0.03000000 \mathrm{sec} \\ 0.0002000 \mathrm{sec} & 0.000\end{array}$

$=== \pm==$ CHAMMEL $f 1$ = $======$

PuC1 $\quad 19 \mathrm{CO}$ usec

$\begin{array}{lr}\text { PL1 } & 7.00 \mathrm{~dB} \\ \text { SFO1 } & 100.6242995 \mathrm{MHz}\end{array}$

$=== \pm \Delta==$ CHAHAEL $L$ f $2======$

$\begin{array}{ll}\mathrm{HUC2} & \mathrm{5H} \\ \mathrm{PCPO} & 90.00 \mathrm{useC}\end{array}$

$\mathrm{PL2} \quad 3.00 \mathrm{~dB}$

21.83

SF02 $400.1319000 \mathrm{MHZ}$

F2 - Pracessing parameters
SI 32768 SF
$5 F-6127744$ HHz

HOW 100.6127744

$\begin{array}{cc}558 & 0 \\ \mathrm{LB} & 1.00 \mathrm{~Hz} \\ \mathrm{~GB} & 0\end{array}$

10 NHA plot parameters

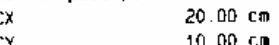

$\begin{array}{ll}\varepsilon_{1} & 10.00 \mathrm{~cm} \\ F_{1} & 240.000 \\ \mathrm{~F} & \mathrm{pom}\end{array}$

$\begin{array}{ll}F 1 & 24147.07 \mathrm{~Hz} \\ \mathrm{FPP} & -10.000 \mathrm{Fm}\end{array}$

$\begin{array}{ll}\mathrm{F}^{2 \mathrm{P}} & -10.000 \mathrm{pP} \\ \mathrm{F} 2 & -1005,13\end{array}$

PFMCM

$\begin{array}{ll}\mathrm{PFMCH} & 12.50000 \mathrm{pDn} / \mathrm{Cm} \\ \mathrm{HZCM} & 1257.65979 \mathrm{~Hz} / \mathrm{cm}\end{array}$ 
$H$ spectrum of

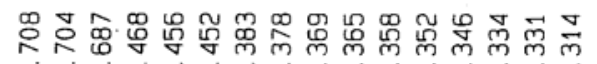

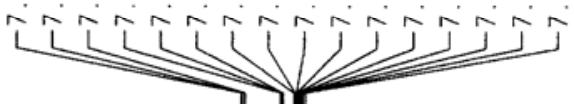

11

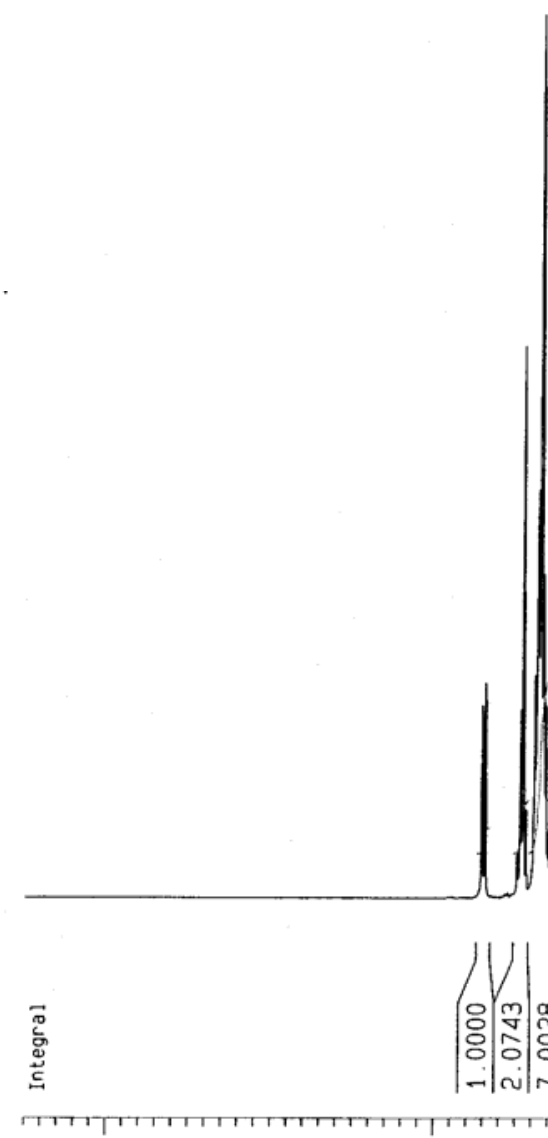

ppm 8
খ

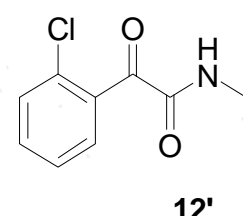

12
Current Data Parameters

NAME 2-Clmedelate

EXPNO
PROCNO

F2 - Acquisition Parameters

Date 20050317

Time 12.14

INSTRUM 5 an

PROBHO $5 \mathrm{~mm}$ BBO BB-1H

PULPROG 2930

TD 1638

SOLVENT CDC13

SWH 5995.204 Hz

$0.365918 \mathrm{~Hz}$

AQ $\quad 1.3664756 \mathrm{sec}$

DW $\quad 83.400$ usec

6.50 usec

$300.0 \mathrm{~K}$

D1 $1.50000000 \mathrm{sec}$

$n z=z=z=$ CHANNEL $f 1=m=z=z$

$\begin{array}{ll}\text { NUC1 } & 1 \mathrm{H} \\ \mathrm{P} 1 & 10.10 \mathrm{use}\end{array}$

PL1 $3.00 \mathrm{~dB}$

SF01 400.1326008 MHz

F2 - Processing parameters

SI 16384

SF $\quad 400.1300091 \mathrm{MHZ}^{2}$

WOW

SSB

$L B$
$G B$

EM

0

$0.10 \mathrm{~Hz}$

1.00

10 NMR plot parameters

CX $\quad 20.00 \mathrm{~cm}$

$\begin{array}{ll}\mathrm{CY} & 10.00 \mathrm{~cm}\end{array}$

F1 $\quad \begin{array}{ll}10.500 \mathrm{Dpm} \\ \mathrm{F}\end{array}$

$\begin{array}{ll}F 1 & 4201.37 \mathrm{~Hz} \\ F 2 P & -0.500 \mathrm{DDm}\end{array}$

F2 $-200.06 \mathrm{~Hz}$

$\begin{array}{lr}\text { PPMCM } & 0.55000 \mathrm{ppm} / \mathrm{cm} \\ \text { HZCM } & 220.07150 \mathrm{~Hz} / \mathrm{cm}\end{array}$ 
C13 spectrum of

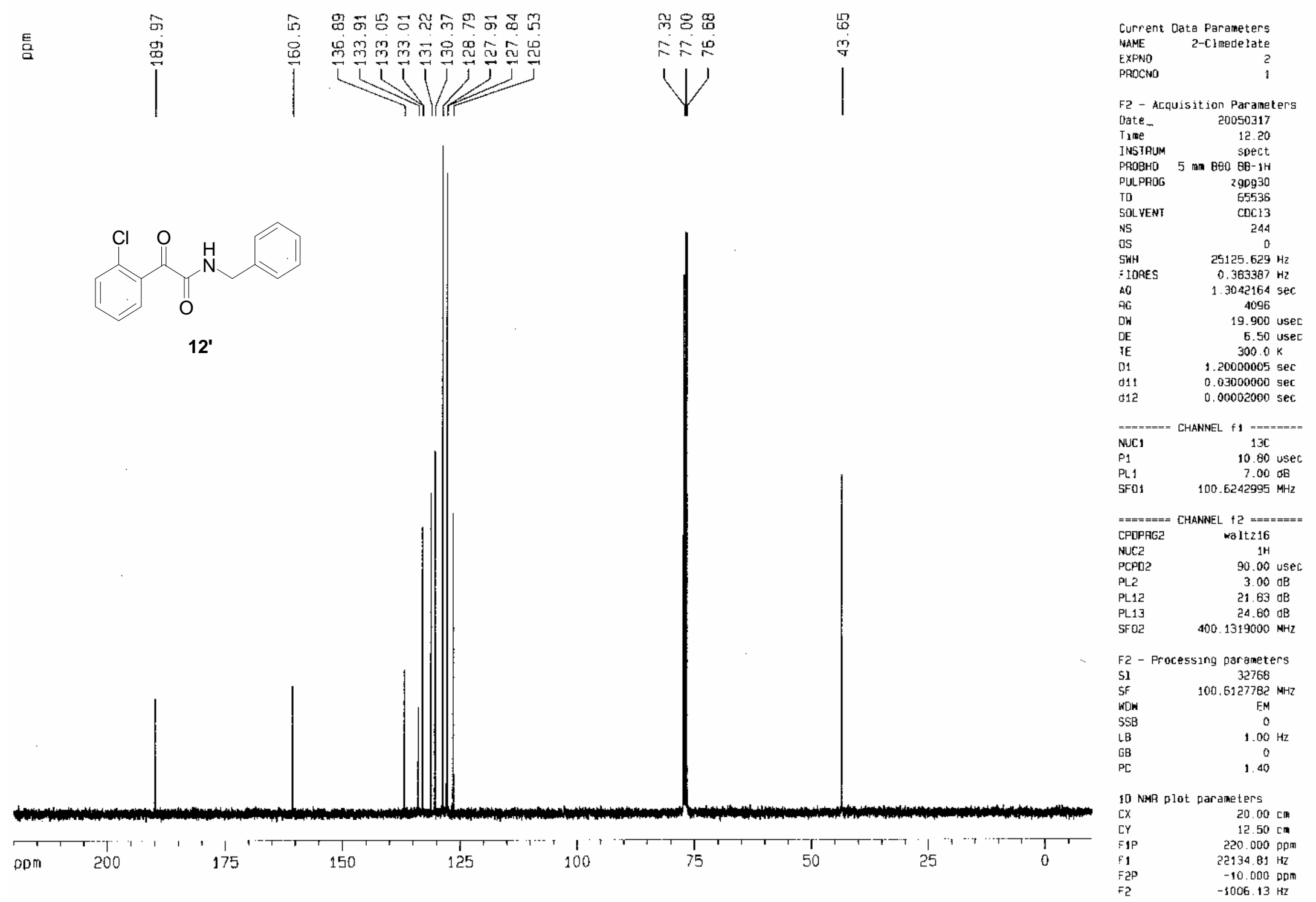




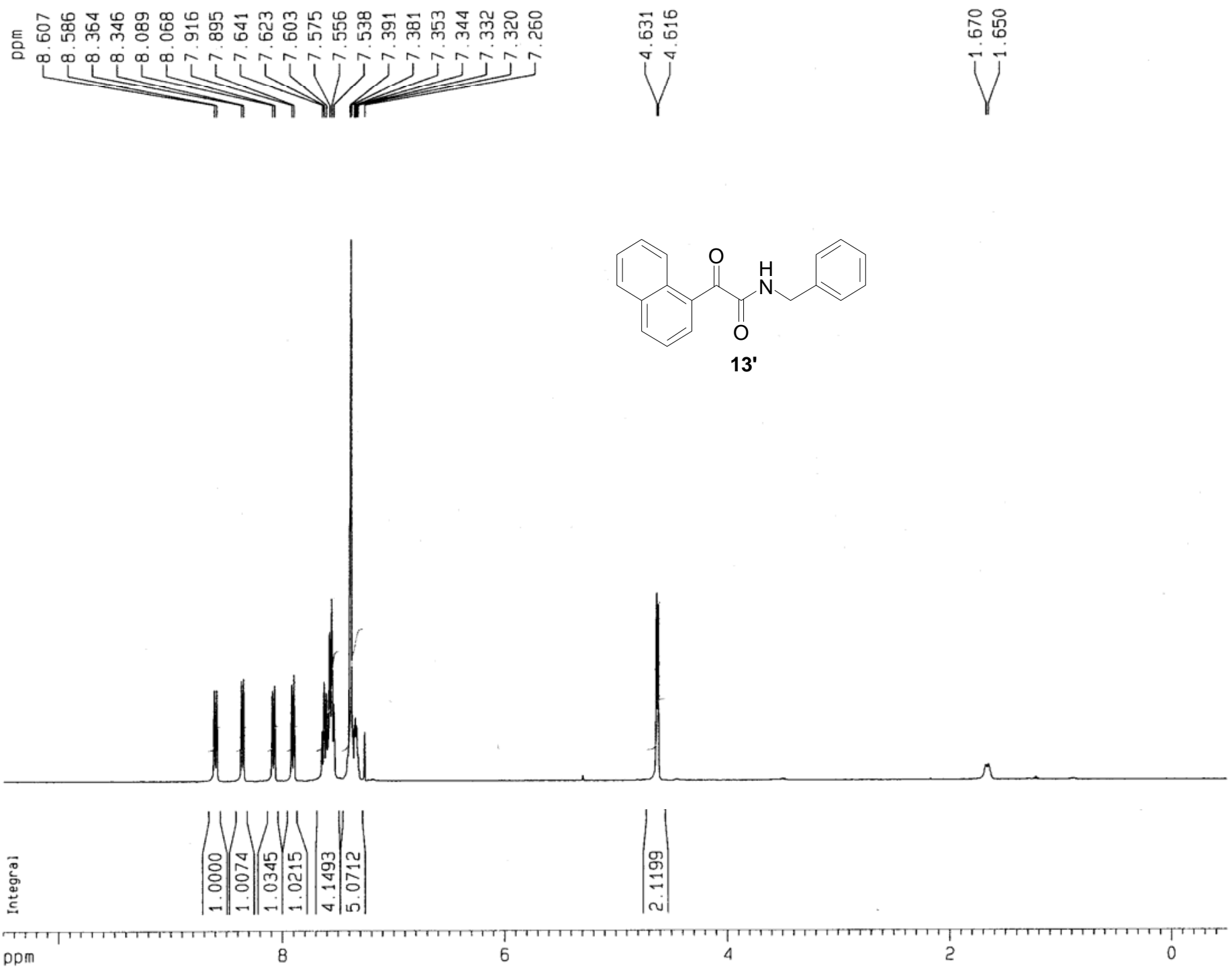

Current Data Parameters

NAME 1-naphthylamida

EXPNO

F2 - Acquisition Parameters

(Dete 20050310

Time

PROBHD spect

PULPROG 5 mI BBO B8-1H

$\begin{array}{lr}\text { PULPROG } & 2930 \\ \text { TD } & 16384\end{array}$

SOLVEN

NS

SWH

AQ $\quad 1.3664756 \mathrm{sec}$

RG $\quad 80.6$

$\begin{array}{rr}\text { OW } & 83.400 \text { usec } \\ \text { DE } & 6.50 \text { usec }\end{array}$

TE $300.0 \mathrm{~K}$

$01 \quad 1.50000000 \mathrm{sec}$

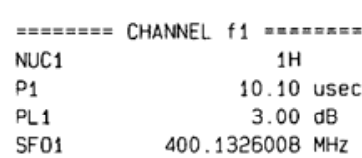

F2 - Processing parameters

$\begin{array}{ll}\text { SI } & 16384 \\ \text { SF } & 400.1300091 \mathrm{MHz}\end{array}$

WDW $\quad 400.1300091$

SSB

GB $\quad 0$

10 NMR plot parameters

CX $\quad 20.00 \mathrm{~cm}$

CY $\quad 9.00 \mathrm{~cm}$

$\begin{array}{ll}\text { F1P } & 10.500 \mathrm{ppm}\end{array}$

$\begin{array}{ll}F 1 & 10.500 \mathrm{DPm} \\ \text { F2P } & -0.500 \mathrm{ppm}\end{array}$

F2 $-200.06 \mathrm{~Hz}$

PPMCM $\quad 0.55000 \mathrm{ppm} / \mathrm{cm}$

$\mathrm{HZCM} \quad 220.07150 \mathrm{~Hz} / \mathrm{cm}$ 
C13 spectrum of

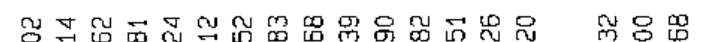
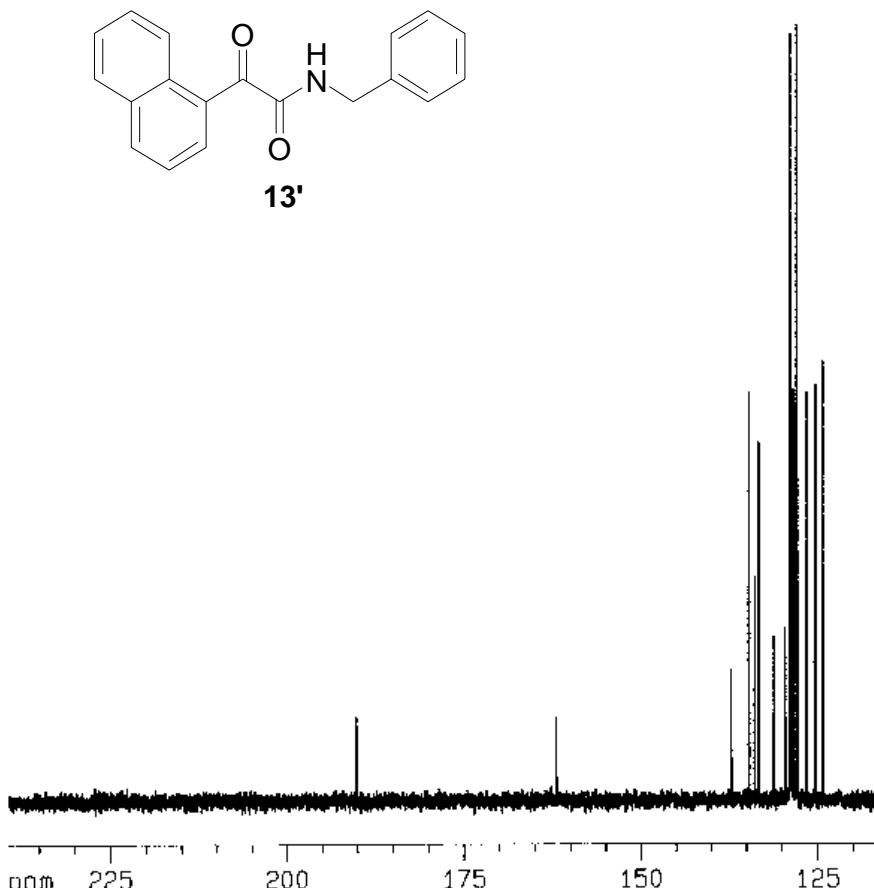

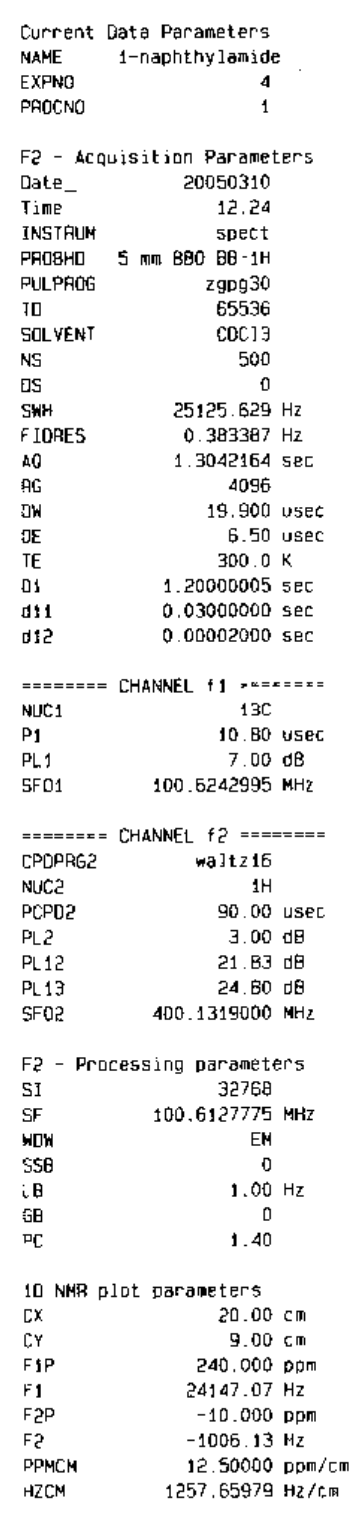


$H$ spectrum of

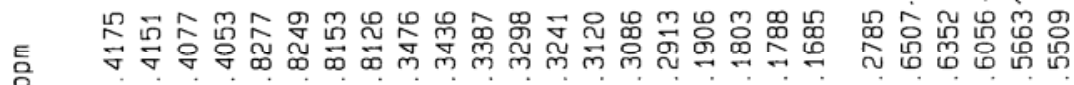

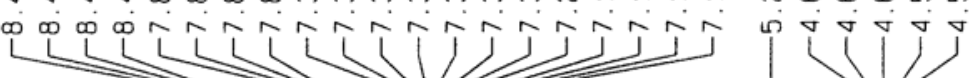
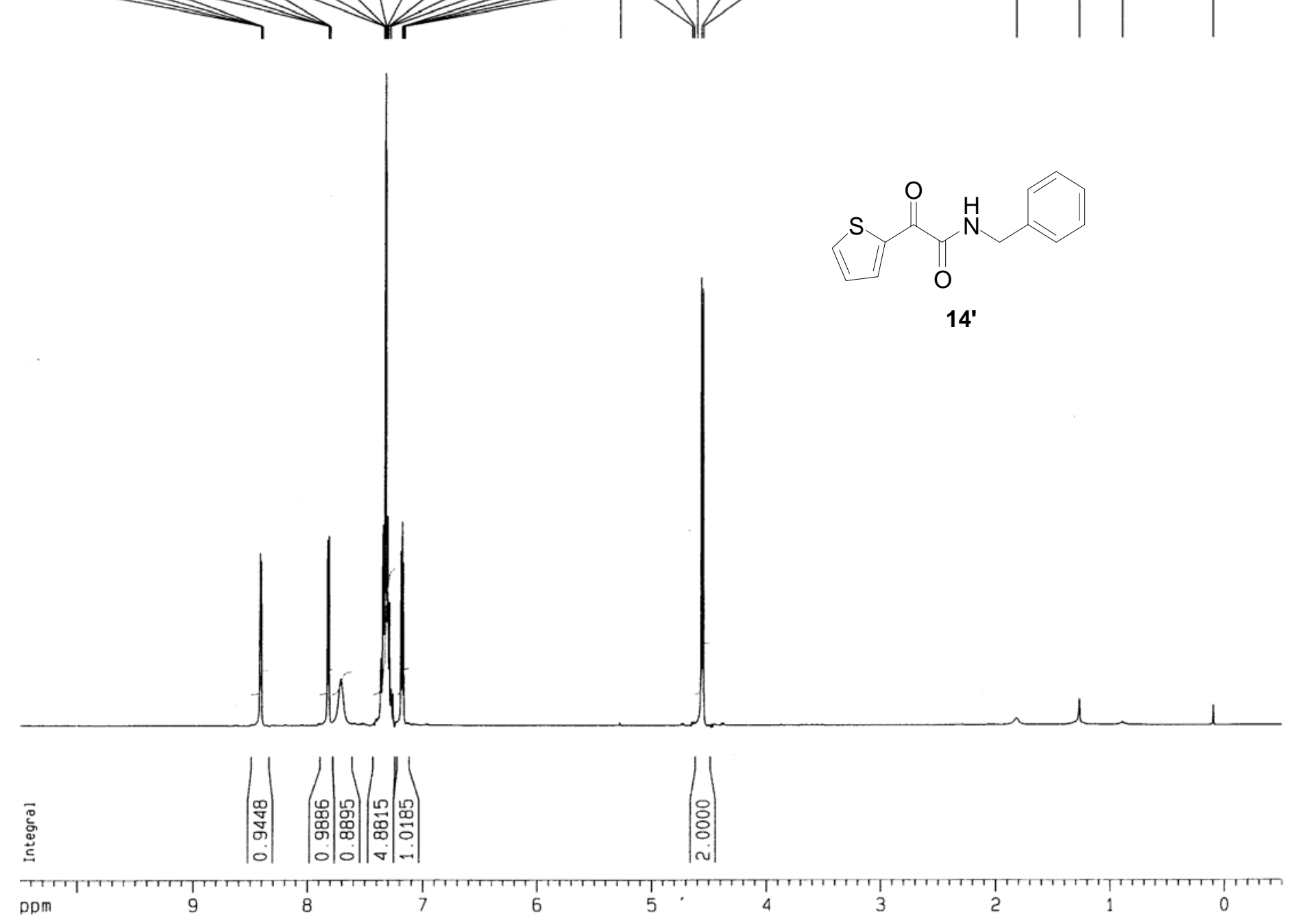

Current Data Parameters NAME oxidation-3 PROCNO

F2 - Acquisition Parameters Date 20050409 $\begin{array}{lr}\text { Time } & 11.01 \\ \text { INSTRUM } & \end{array}$ INSTRUM spect PROBHD $5 \mathrm{~mm}$ BBO BB-1H PULPROG $\begin{array}{ll}\text { TD } & 16384 \\ \text { SOLVENT } & \text { COC13 }\end{array}$ NS 16

SWH $\quad 5995.204 \mathrm{~Hz}$

$0.365918 \mathrm{~Hz}$

AQ $\quad 1.3664756 \mathrm{sec}$

RG $\quad 80.6$

DW $\quad 83.400$ usec

DE 6.50 use

TE $300.0 \mathrm{~K}$

$01 \quad 1.50000000 \mathrm{sec}$

$=z=z= \pm=$ CHANNEL $f 1=z=z= \pm=$ NUC1 $1 \mathrm{H}$

$\begin{array}{lr}\text { P1 } & 10.10 \text { usec } \\ \text { PL.1 } & 3.00 \mathrm{~dB}\end{array}$ SF01 $400.1326008 \mathrm{MHz}$

F2 - Processing parameters

SI 16384

SF $\quad 400.1300091 \mathrm{MHz}$

WDW

SSB

LB

$\mathrm{CX}$. $20.00 \mathrm{~cm}$

CY $\quad 20.00 \mathrm{~cm}$

FIP $\quad 10.50 \mathrm{~cm}$

F1 $\quad \begin{array}{ll}10.500 \mathrm{Dpm} \\ \mathrm{F}\end{array}$

$\begin{array}{ll}F 2 P & -0.500 \mathrm{ppm}\end{array}$

$\begin{array}{ll}\text { F2 } & -200.07 \mathrm{~Hz} \\ \text { PPMCM } & 0.55000 \mathrm{pPm} / \mathrm{Cm}\end{array}$

$\mathrm{HZCM} \quad 220.07152 \mathrm{~Hz} / \mathrm{cm}$ 
C13 spectrum of
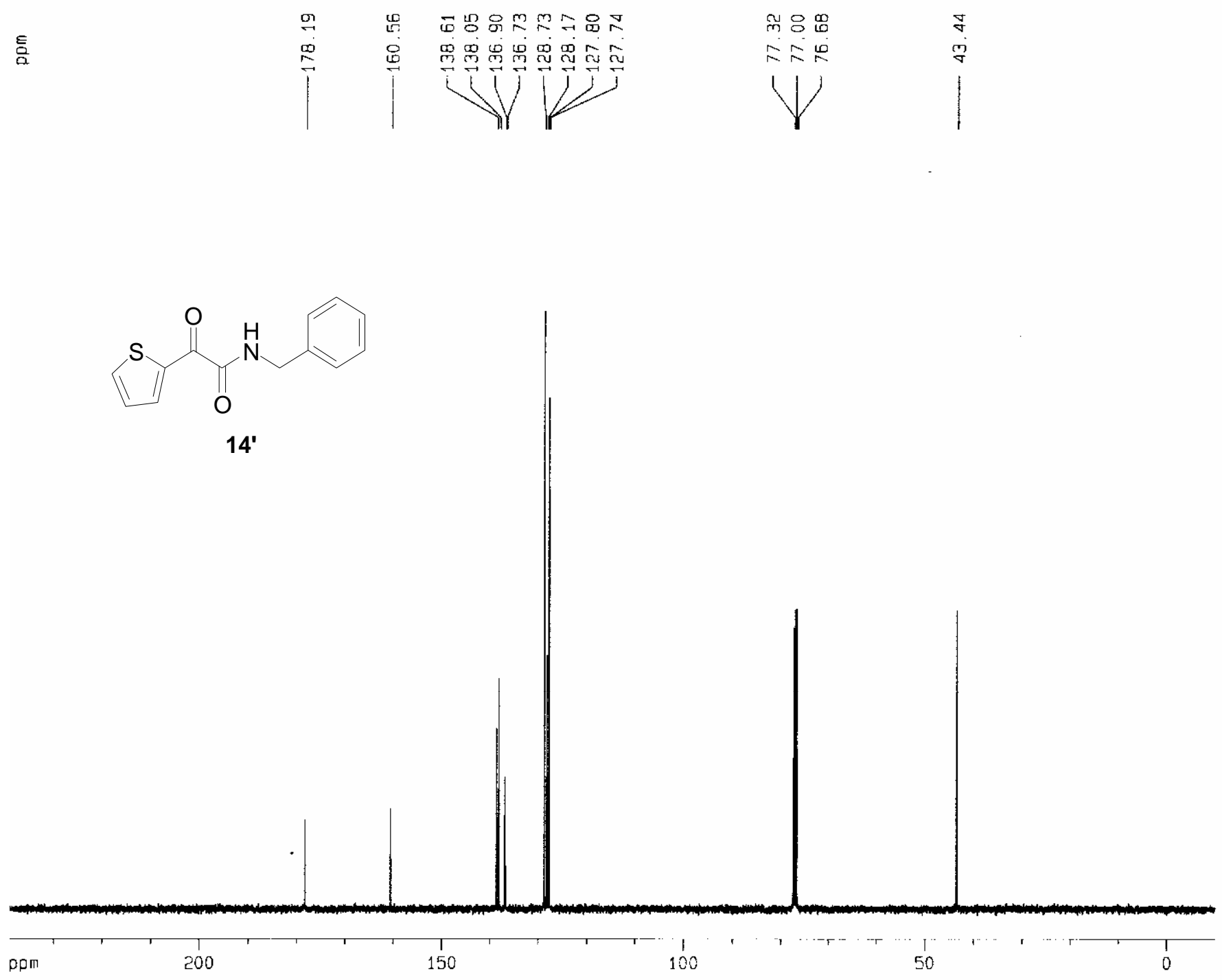

arrent Data Parometers

NAME DPNG DX dation-3

PROCNO

$F 2$ - ACquisst ion paraneter

Date

INSTRIM

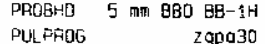

$\begin{array}{ll}290930 \\ \text { TD } & 65535\end{array}$

$\begin{array}{lr}\text { SOLVENT } & \text { CDC13 } \\ \text { MS } & 128 \\ 05 & 0\end{array}$

5Hi- $25135.629 \mathrm{~Hz}$

FIÜRES $\quad 0.383987 \mathrm{~Hz}$

A6 $1.3042164 \mathrm{sec}$

DH $\quad 19.900$ user

TE $300.0 \mathrm{~K}$

$01-1.20000005 \mathrm{sec}$

11?

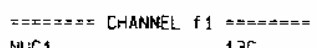

$\begin{array}{ll}\text { NuC1 } & 136 \\ D_{1} & 10.80 \text { usec }\end{array}$

$100.00 \mathrm{~GB}$

=

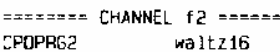

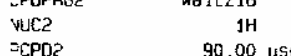

$=\mathrm{CPOC}$
$=\mathrm{L2}$

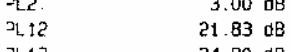

SF02 $400.13+9000$ MHX

F2 - Processing paraneters

SI Protessing paraneters

iF $\quad 100$

$\begin{array}{cc}{ }_{10} & \text { EN } \\ \text { SSE } & 0 \\ B & 1.00 \mathrm{~Hz}\end{array}$

$\begin{array}{lr}\mathrm{B} & 1.00 \\ \mathrm{~GB} & 1.00 \\ \mathrm{PC} & 1.0\end{array}$

10 MMP diot parameters

$\begin{array}{ll}c x & 20.00 \mathrm{~cm} \\ \mathrm{Cr} & 10.00 \mathrm{~cm}\end{array}$

$\begin{array}{ll}\text { FiP } & 10.00 \mathrm{~cm} \\ \text { Fi } & 240.000 \mathrm{pDm}\end{array}$

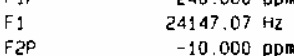

$\begin{array}{ll}F 2 & -10.000 \mathrm{pPm} \\ F 2 & -1006.13 \mathrm{hI}\end{array}$

HZCM $\quad 1257.65979 \mathrm{HzZ} / \mathrm{cm}$ 
$H$ spectrum of
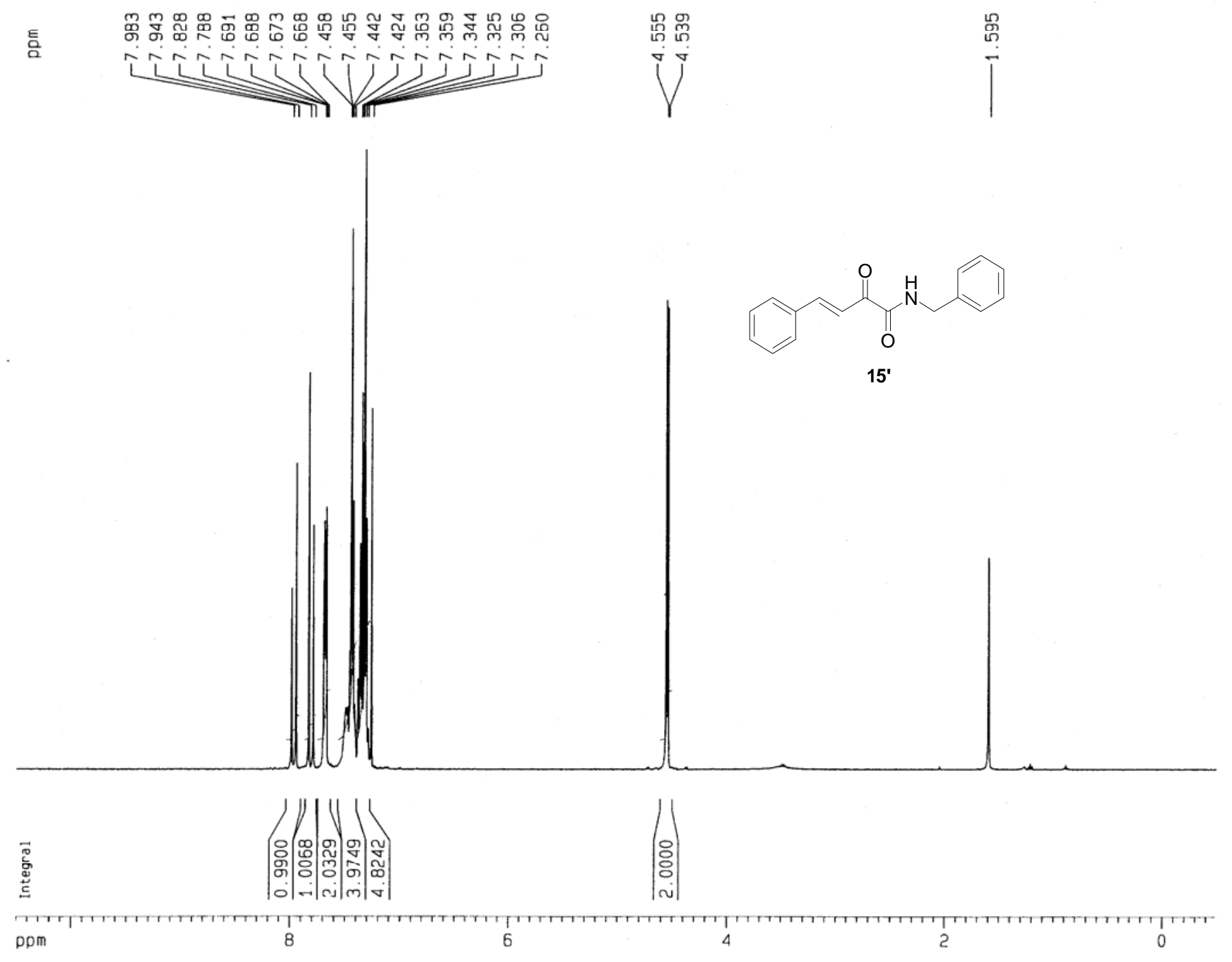

Current Data Parameters

CinamylBnamic

PROCNO

F2 - Acquisition Parameters

$\begin{array}{lr} & 20050317 \\ \text { Date } & 12.35\end{array}$

12.35

PROBHO $5 \mathrm{~mm}$ BBO BB-1H.

PULPROG 2930

16384

SOLVEN

$\begin{array}{ll}\text { OS } & 0 \\ \text { SWH } & 5995.204 \mathrm{~Hz}\end{array}$

$0.365918 \mathrm{~Hz}$

AQ $\quad 1.3664756 \mathrm{sec}$

$\begin{array}{lr}\text { RG } & 322.5 \\ \text { DW } & 83.400 \text { usec } \\ \text { DE } & 6.50 \text { usec }\end{array}$

D1 $\quad 1.50000000 \mathrm{sec}$

$\begin{array}{lr}== \pm== \pm=\text { CHANNEL } f 1 \quad== \pm== \pm= \\ \text { NUC1 } & 1 \mathrm{H} \\ \text { P1 } & 10.10 \mathrm{useC} \\ \text { PL1 } & 3.00 \mathrm{~dB} \\ \text { SF01 } & 400.1326008 \mathrm{MHz}\end{array}$

F2 - Processing parameters

$\begin{array}{ll}\text { SI } & 16384 \\ \text { SF } & 400.1300095 \mathrm{MHz}\end{array}$

WOW

SSB

LB

PC

1D NMR plot parameters

CX $20.00 \mathrm{~cm}$

$\begin{array}{ll}\text { CY } & 10.50 \mathrm{~cm} \\ \text { F1P } & 10.500 \mathrm{DDm}\end{array}$

$\begin{array}{ll}F 1 & 4201.37 \mathrm{~Hz} \\ \text { F2P } & -0.500 \mathrm{DPn}\end{array}$

F2 $\quad-0.500 \mathrm{pDm}$

PPMCM $\quad 0.55000 \mathrm{pDm} / \mathrm{cm}$ 
C13 spectrum of
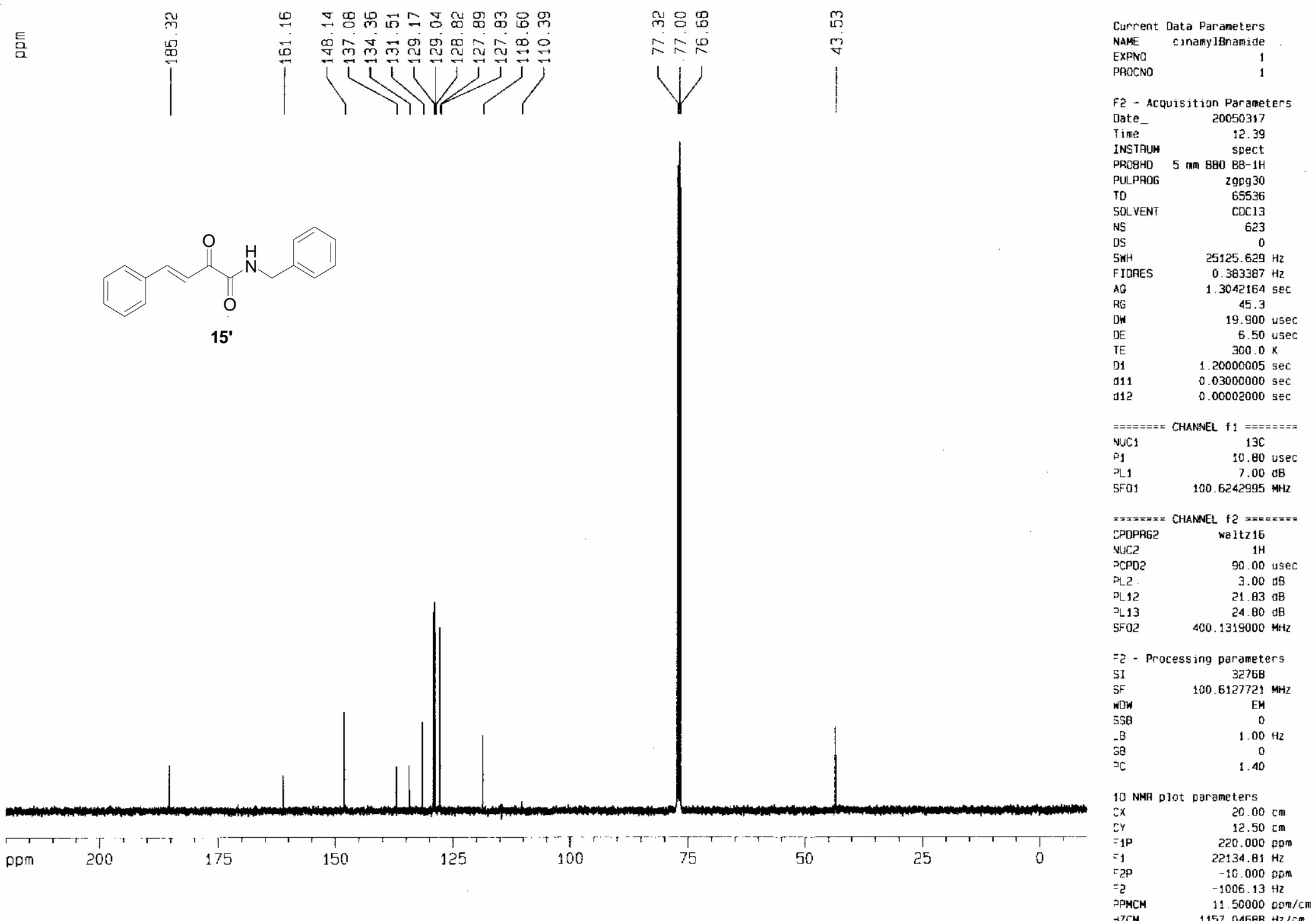
H spectrum of

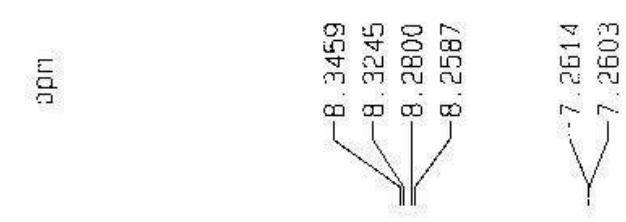

O
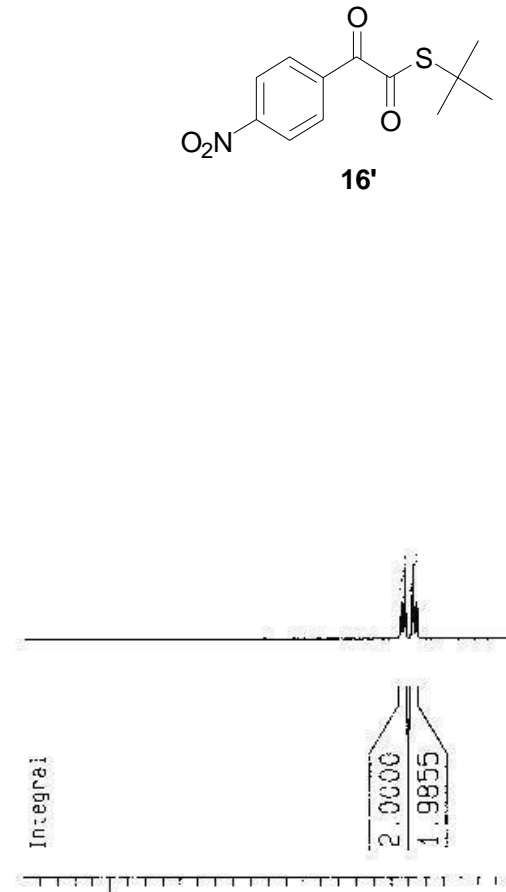

ppm

di.

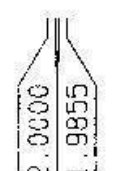

용
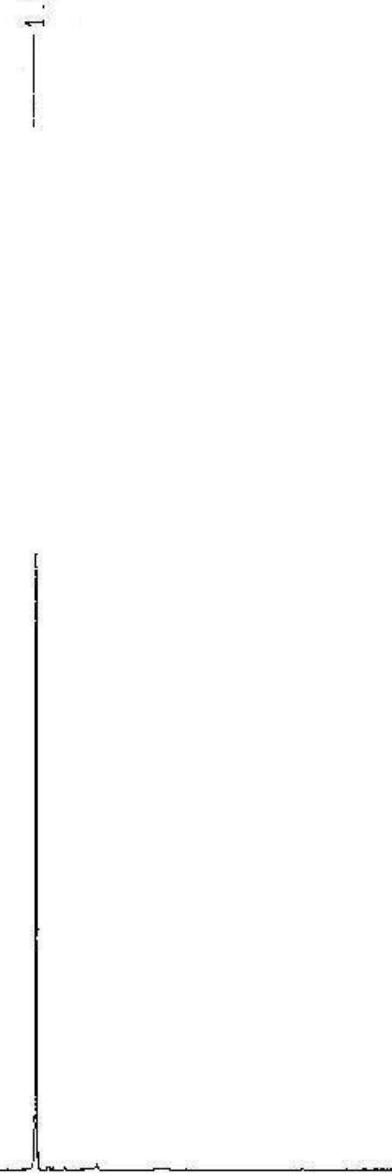

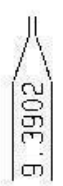

m.
Namt. 5 am 4-N1tro ket

EXPNO

F2 - Acquj5itjon Parameters

Date_ 20060314

Time $\quad: 8.55$

PAOEHD 5 mo HeO SB-iH

PUL.FROG $\quad$ zg30

TD

SOLVENT

N5

SWH $\quad 5995.204 \mathrm{~Hz}$

FICRES $\quad 0.365918 \mathrm{itz}$

$\triangle 901.3664756 \mathrm{sec}$

AG E45.1

$\begin{array}{lr}\text { DW } & 83.400 \text { ปsec } \\ \text { DE } & 6.50 \text { isec }\end{array}$

IE $300.0 \%$

D1 $1.50000000 \mathrm{sec}$

$======$ CHANNEL $\quad \mathrm{i}:====--=$

P1 :0.10 user

$\begin{array}{lr}\text { PL1 } & 3.00 \mathrm{~dB} \\ \mathrm{SF} & 400 \quad 1325003 \mathrm{MHz}\end{array}$

Pingers

Fa - Processing parameter

SF $\quad 400.1300091 \mathrm{MHz}$

WCW $\quad F M$

LB $\quad 0.10 \mathrm{~Hz}$

GB

1C. NMR f flet parameters

CX

CX
CY

$\begin{array}{ll}\text { CY } & 6.50 \mathrm{~cm} \\ \text { F1F } & 10.500 \mathrm{ogm}\end{array}$

F1 $\quad 0.500 \mathrm{Dpm}$

FEP $\quad-0.500 \mathrm{DLm}$

$=2 \quad-200.05 \mathrm{~Hz}$

$\begin{array}{lr}\mathrm{PPMCM} & 0.55000 \mathrm{ppm} / \mathrm{cm} \\ \mathrm{hZCM} & 220.07150 \mathrm{~Hz} / \mathrm{cm}\end{array}$ 
¿13 spectrum of
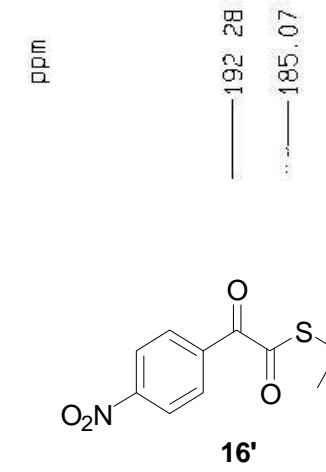

$16^{\prime}$
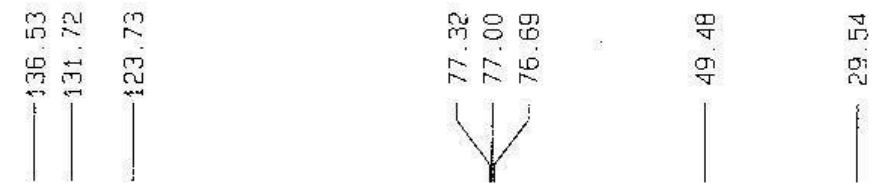

\section{.}

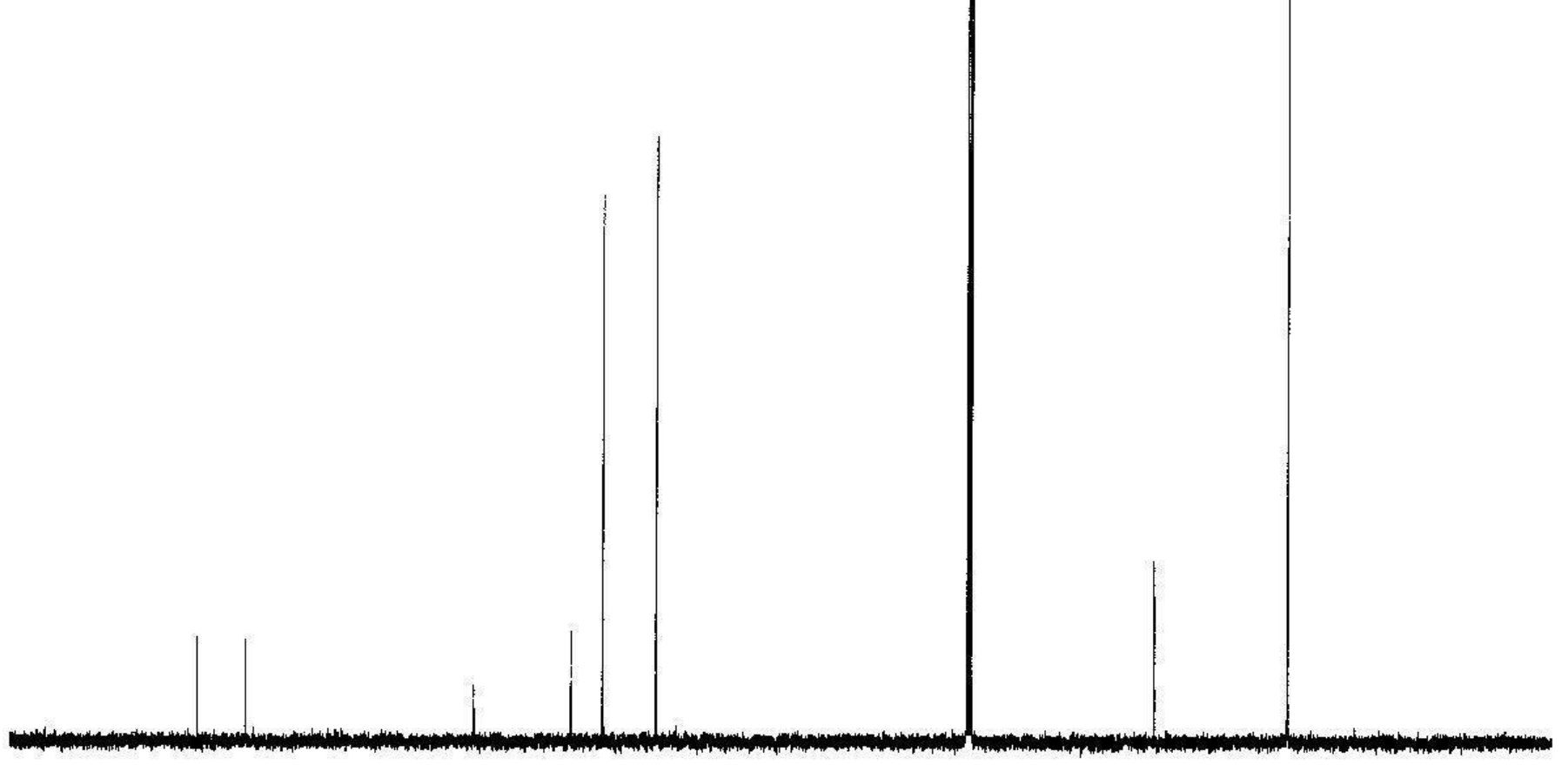

i $T \cdot T-T$

pp

200

150
125
100

ऽ0

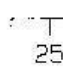

$T$
25

Current Datō Farameters

NAME Säll. $1 \cdot{ }_{1}$

DROCMO

-2 - Acquisition Paramctiors

Jate

Time-

spect

ग्र

TO

SOL

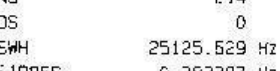

AQ $\quad 0.383397 \mathrm{~Hz}$

AG $\quad 6192$

$\begin{array}{lr}\text { DK } & 19.900 \text { usec } \\ \text { DE } & 6.50 \text { usec }\end{array}$

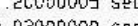

$0.00002000 \mathrm{sec}$

$======$ CHANNEL $11=======$

$\begin{array}{ll}\text { VuC. } 136 \\ \text { P1 } & 10.80 \text { usec }\end{array}$

PL1 $\quad 7.00 \mathrm{aB}$

Ta

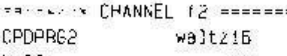

$\begin{array}{ll}\text { NUC2 } & 1 \mathrm{H} \\ \text { PCPD2 } & 90.00 \text { Use }\end{array}$

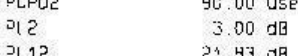

ग.1.13 24.93013

SFC2 $400.1319000 \mathrm{M}=12$

$=2$. Processung parameter:

SI 32760

SF $\quad 100.6 \pm 27721 \mathrm{MHI}$

$5 S 8$

258
$G B$
$G B$

$\quad 1.0 \mathrm{OC}$

IC NWP Fint ooranters

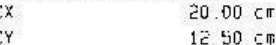

$20.000 \pi$
1050
520.000

$22134.81 \mathrm{~Hz}$

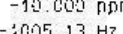

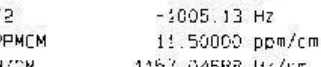


H spectrium of
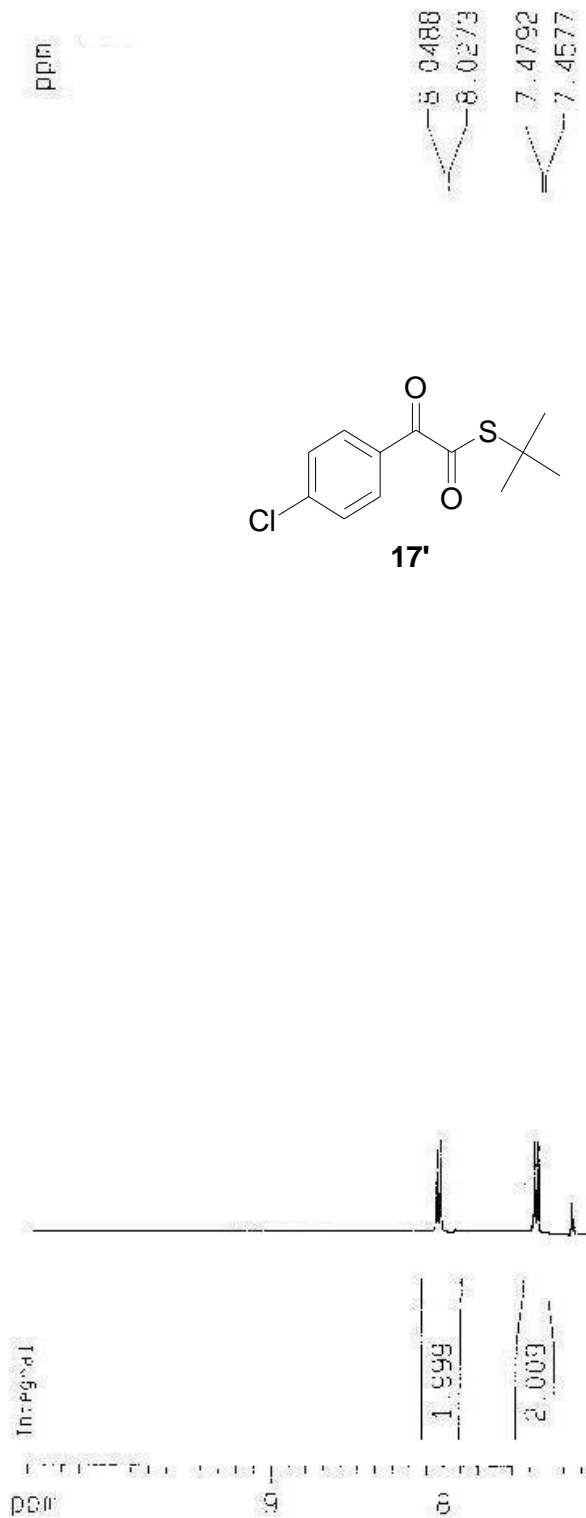

ह

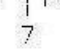

7
突

บ새는

-xFin

4-C: the $k \in t$

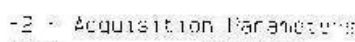

Zote_ 2006

2000,16
11.05

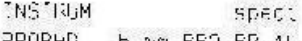

JULPREG

15 \$536

(15)

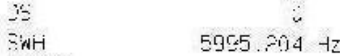

-TCies

$401.3664756=12$

76 - $25 \mathrm{E}$

Ill B 3.200 usec 6. 5 (i usec 50000000 :ier

7

ANREL f1 $1--1====$ WL1 $1 \mathrm{H}$

i1 10.10 บsec Stm1 $\quad 4.00 \mathrm{~dB}$

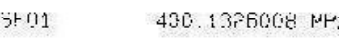

-2. payessing paranelers

110394

3 T09.1300095 MII

$\begin{array}{ll}\mathrm{NCM} & \mathrm{EM} \\ \because \mathrm{GE} & \end{array}$

$\begin{array}{ll}3 \mathrm{~B} & 0 \\ \mathrm{~B} & 0.10 \mathrm{~Hz}\end{array}$

उB

P 1.00

II N.TP n: nt 93-ameigms

$\begin{array}{ll}\because & 0.00 \mathrm{~cm} \\ 0 & 10.5 \mathrm{~cm}\end{array}$

$-12 \quad 10.50 \mathrm{~nm}$

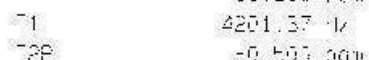

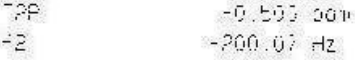

गNCM $55.000 \mathrm{nMm} / \mathrm{Cm}$

17CM 22\% $\mathrm{C}: 15 \mathrm{~Hz} \mathrm{~Hz}$ 
$013 \operatorname{sectran} 0$
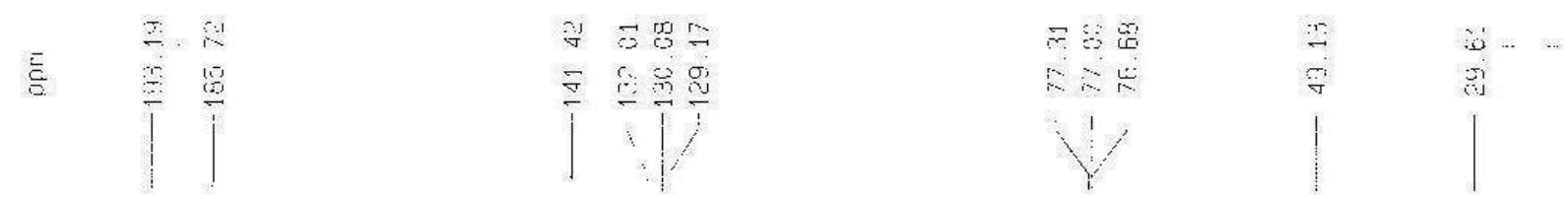

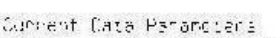

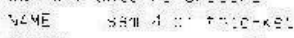

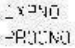

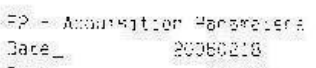

T:He -

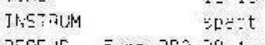

TI:CE-AL 5 r:m FFo उe 1 .

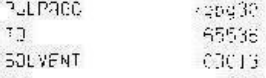

25125, है: $\mathrm{He}$

$0.363387 \mathrm{H}$
$1.3042154 \mathrm{sec}$

$\mathrm{S}$

$\mathrm{Cl}$

17

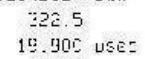

$\begin{array}{rl}30.0 & 0\end{array}$

30.0

1. $0.3000000=0$ 6. $0000000 \mathrm{FeC}$

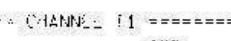

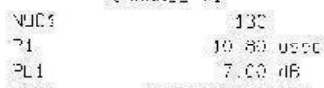

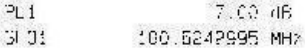

CPIOFG? CHANMLL $: \bar{z}======$

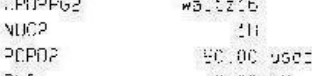

गद 10 ग

?. 13 24.

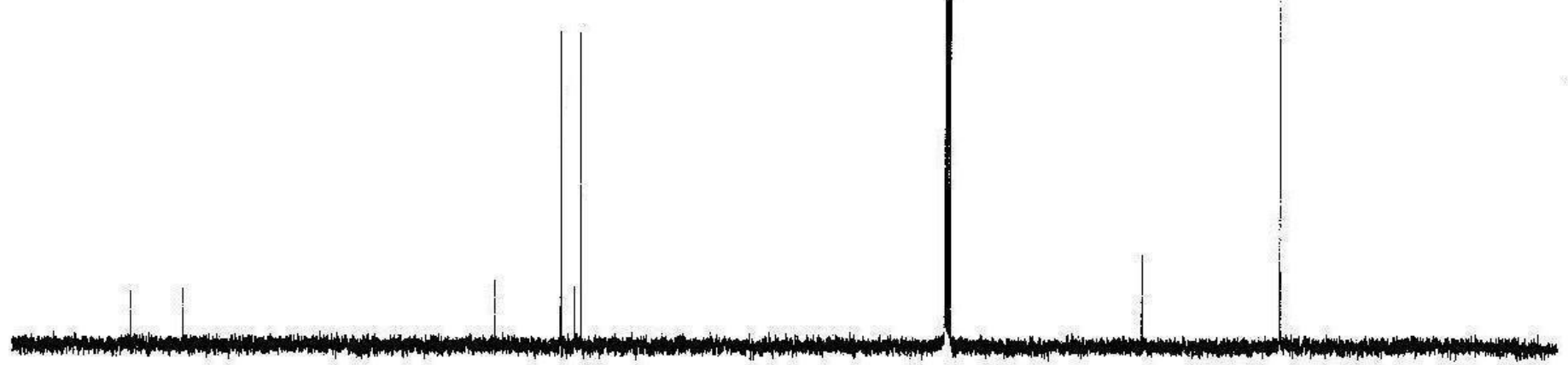

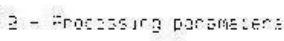

$=1230$

in

B $1.6 \mathrm{H}$

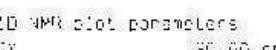

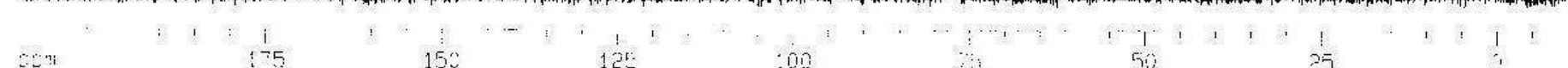

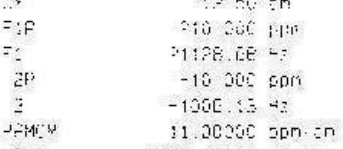


$\overline{8}$

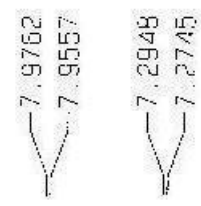

(1)

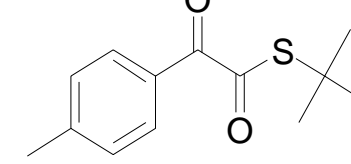

18'

\section{H spert.rium of}
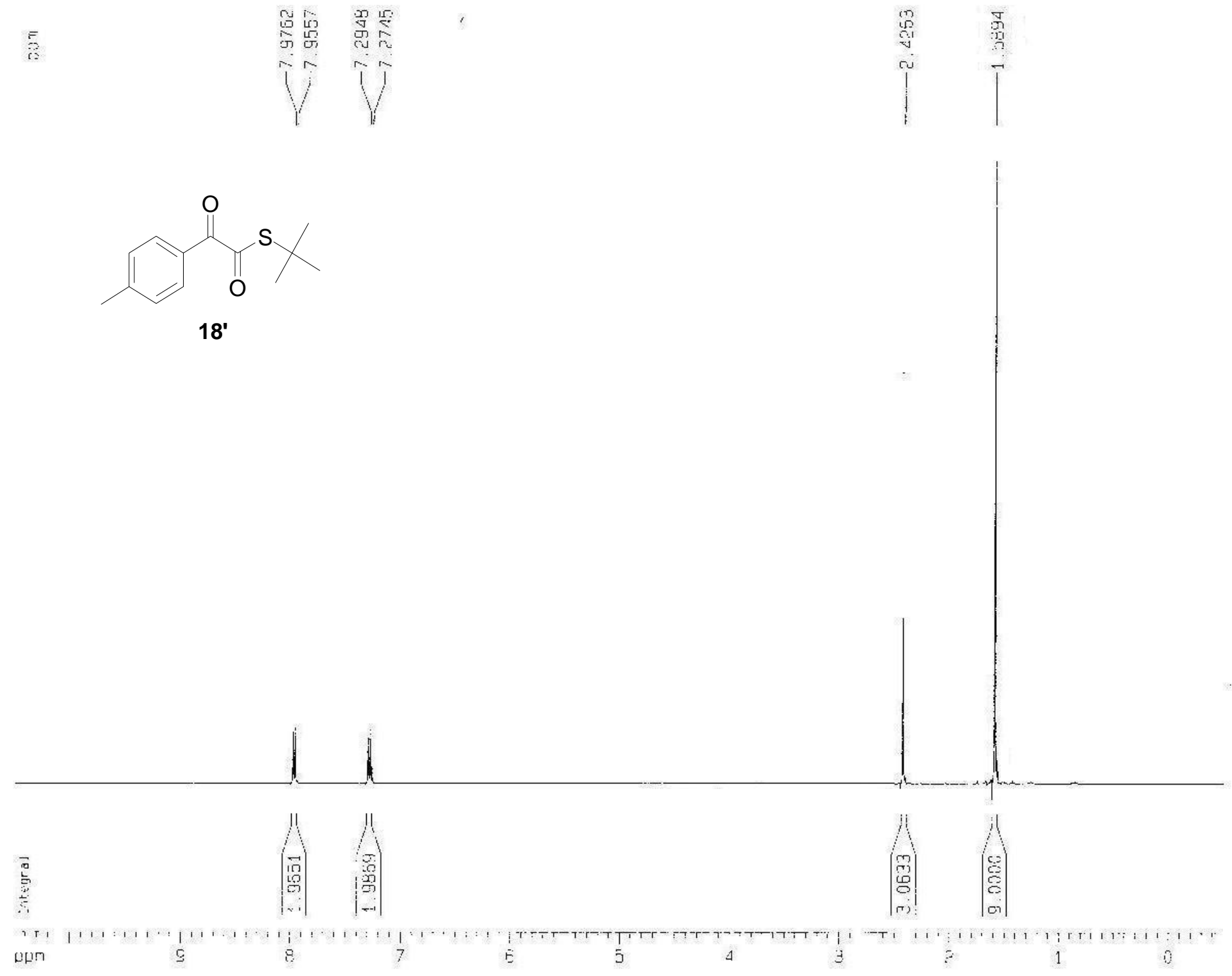

VAME

EXINO
PRSCNO

1
1
1

F2. Acquisutim anaters

Date Z0

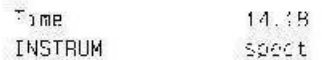

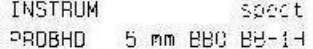

P.

Tn

50. VEN

施

Is

उWH $5995.204 \mathrm{~Hz}$

- IDRES O. 365912 Hz

$45 \quad 1.3664 \% 56$ SeL

Is $\quad 226$

DE $\quad$ E. 50 use

TE $300.0 \mathrm{~K}$

J1 $1.50000000 \mathrm{sec}$

$---==-=$ CHAMIKEL $+1=======$
NUC1
IH

$\begin{array}{ll}\text { गUCI } & 1 \mathrm{H} \\ \mathrm{J}_{1} & 10.10 \mathrm{USEL}\end{array}$

$\begin{array}{ll}\rho_{1} & 10.10 \text { use } \\ 3_{1} & 3.00 \mathrm{~dB}\end{array}$

SF01 $\quad 400.1326005 \mathrm{M} M-12$

-2 - Processing parameteró

SI 16394

SF $\quad 460 . \leqslant 300095 \mathrm{MHz}$

vinin

ss

GB

EM

c. 0.00

10 NMP filot paralinti..';

ix $20.00 \mathrm{~cm}$

$\begin{array}{lc}20 & 19.50 \mathrm{~cm} \\ =1 \mathrm{P} & 10.500 \mathrm{cpm}\end{array}$

$4.91 .37 \mathrm{~Hz}$

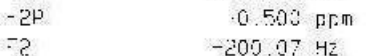

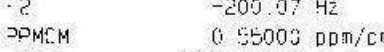

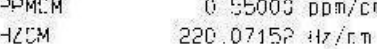



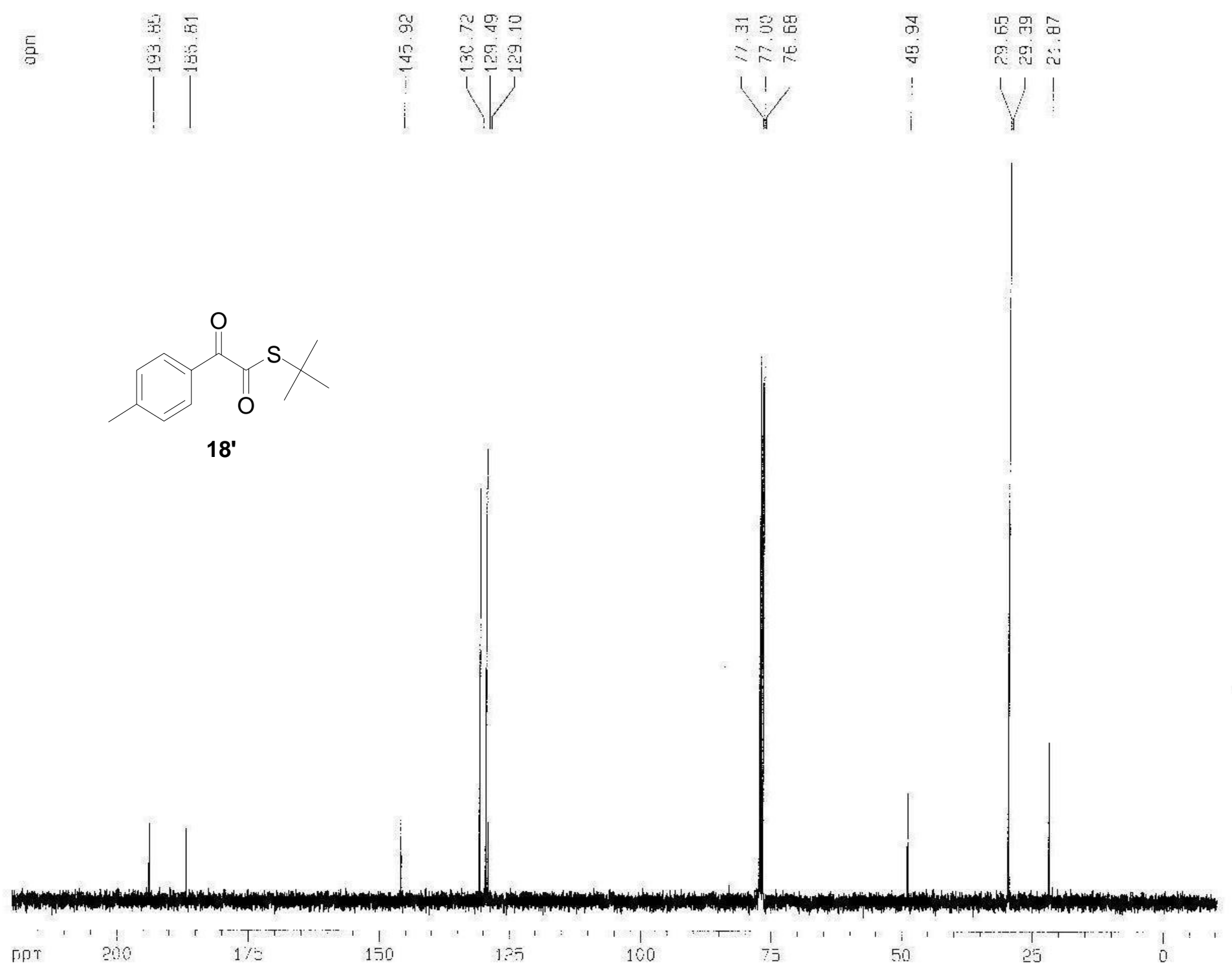

1) NHF. nint paramzters
$2 x$
20.20 cill

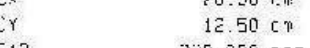

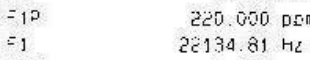

$\begin{array}{ll}=1 & 20134.81 \mathrm{HL} \\ =2 \mathrm{p} & -10.357 \mathrm{prm}\end{array}$

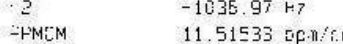

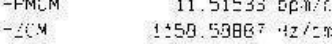


$H$ spectrum of

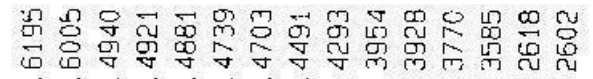
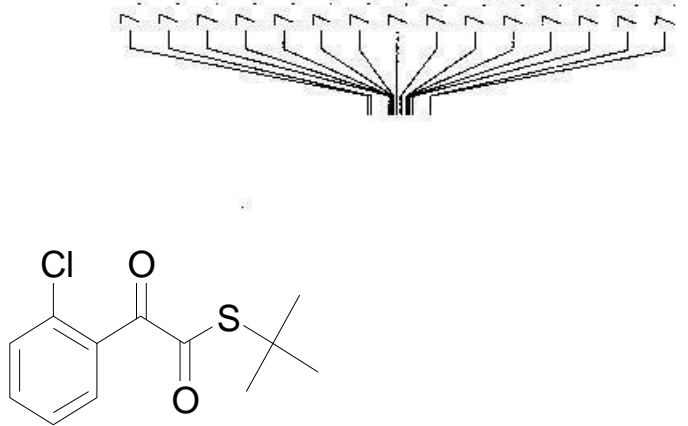

19 wherit vata rarameters VAME Sam-2-C1-H-Ket EXPNO

PROCNO

=2. Acqujsitior Parameters Date_ 2006052B Time 17.35 INSTRUM spect गROBH $5 \mathrm{~mm} B 80 \mathrm{BB}-1 \mathrm{H}$ JULPROG 2930 TO 163394 SOLVEN1
NS DS SWH $\quad 5995.204 \mathrm{~Hz}$ FIDRES $\quad 0.365918 \mathrm{~Hz}$ $A B \quad 1.36647565$ 7G 228.1 $\begin{array}{rr}J W & 83.400 \text { usec } \\ J E & 6.50 \text {. Jsec }\end{array}$ TE $\quad 300.0 \mathrm{~K}$ $01 \quad 1.50000000 \mathrm{sec}$ $=======$ CHANNEL $f 1======$
NUC1 $\begin{array}{lll}\text { NUC1 } & 1 \mathrm{H} \\ 31 & 10.10 \text { LSEC }\end{array}$ SF $01 \quad 400 \$ 326008 \mathrm{MH}$

-2 - Processing parameters SI \$6364 SF $\quad 400.1300080 \mathrm{MHz}$ WDW LB $\quad 0.10 \mathrm{li} \%$ $\begin{array}{ll}G B & 0 \\ D E & 1.00\end{array}$

10 NMR plot parameters

Ex $20100 \mathrm{~cm}$ $\mathrm{cr} \quad 10.500 \mathrm{~cm}$ F1P $\quad 10.500 \mathrm{ppm}$ F1 $4201.37 \mathrm{~Hz}$

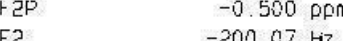
PPMCM $\quad 55000 \mathrm{PDm} / \mathrm{Cm}$

H८CM $22007152 \mathrm{~Hz} / \mathrm{cm}$

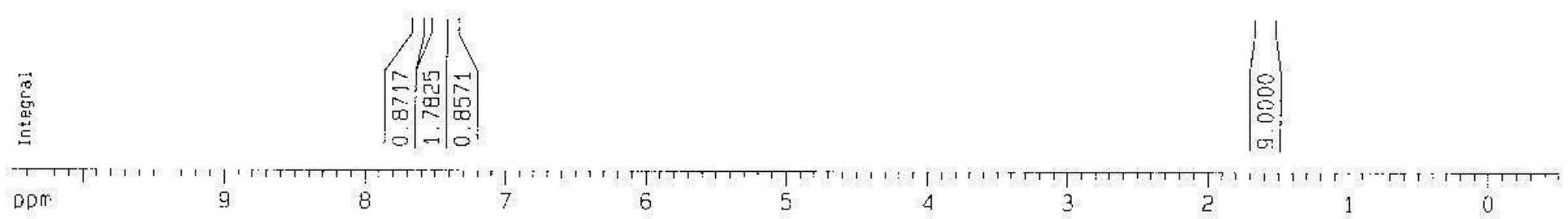

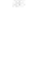


C13 spectrum of

틈

Cl O

$\mathrm{S}$

O

$19 '$

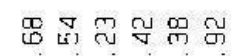

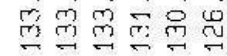

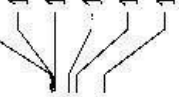

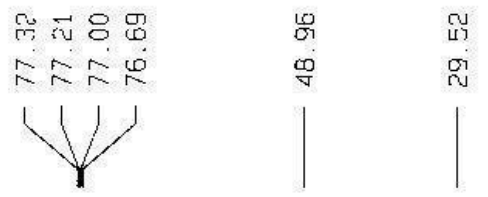

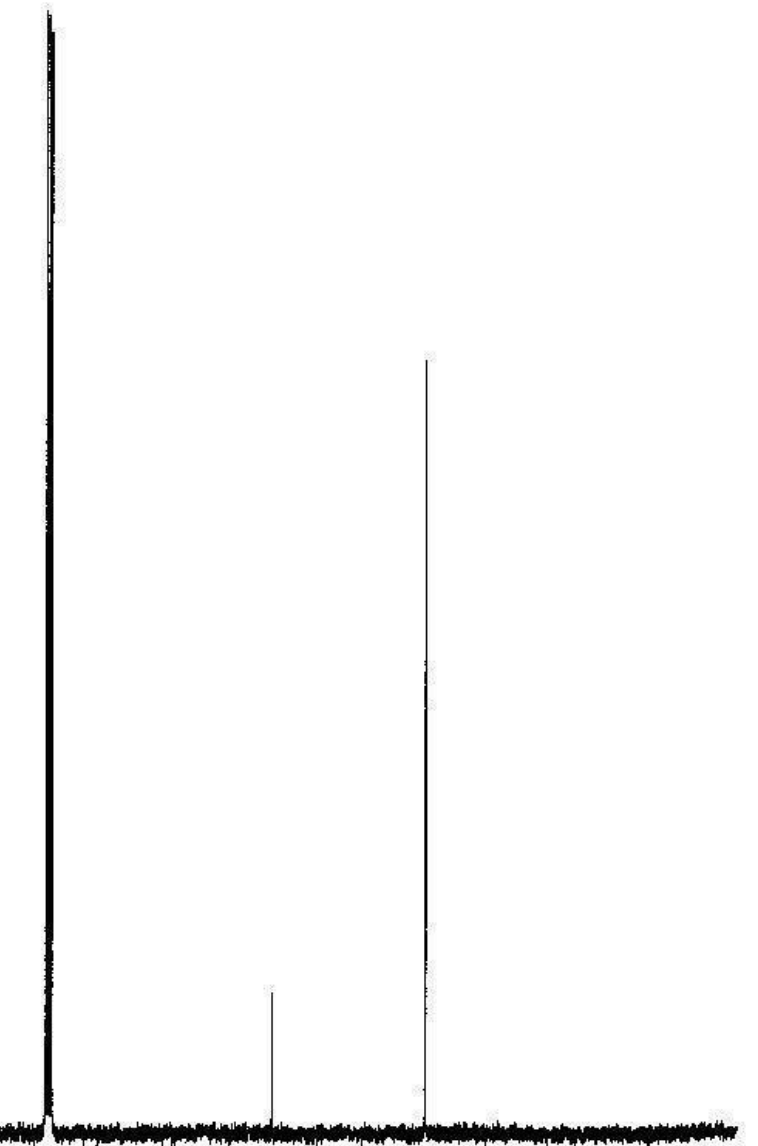

$i$
75

1
25

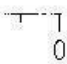

175

150
125
100

$3 x$
$z y$
$z y$ $=1$
$=1$
$=1$ $\rightarrow 7$

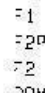

$$
\begin{aligned}
& \text { Current Lata Paralmeter's } \\
& \text { WAME } 5 \bar{a} \mathrm{~m}-2-\mathrm{CH}_{\mathrm{i}}-\mathrm{HT}-\mathrm{Ke} \\
& \text { EXPNO }
\end{aligned}
$$

NMR elot parame'ers

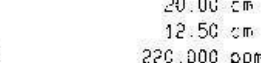

2.06 .000
22134

-10.200 p

$-1005 . \because \mathrm{Hz}$

$11.50000 \mathrm{plom} / \mathrm{cm}$ 
H spectrum of

틈
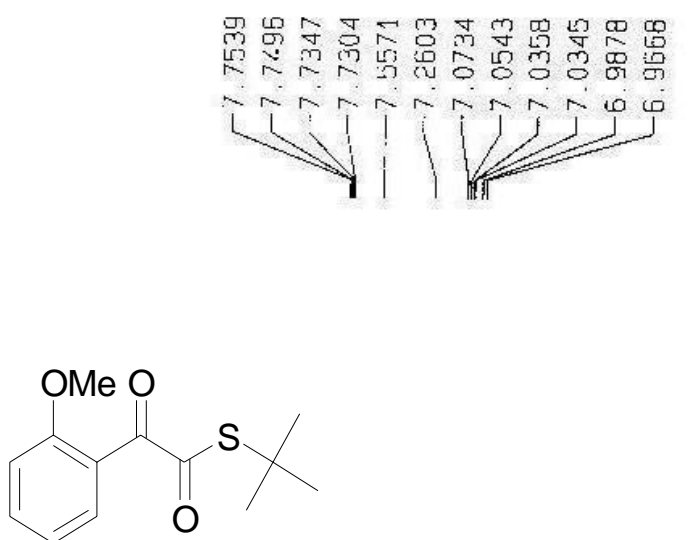

20'

\section{$\stackrel{\substack{n \\ 0}}{m}$}
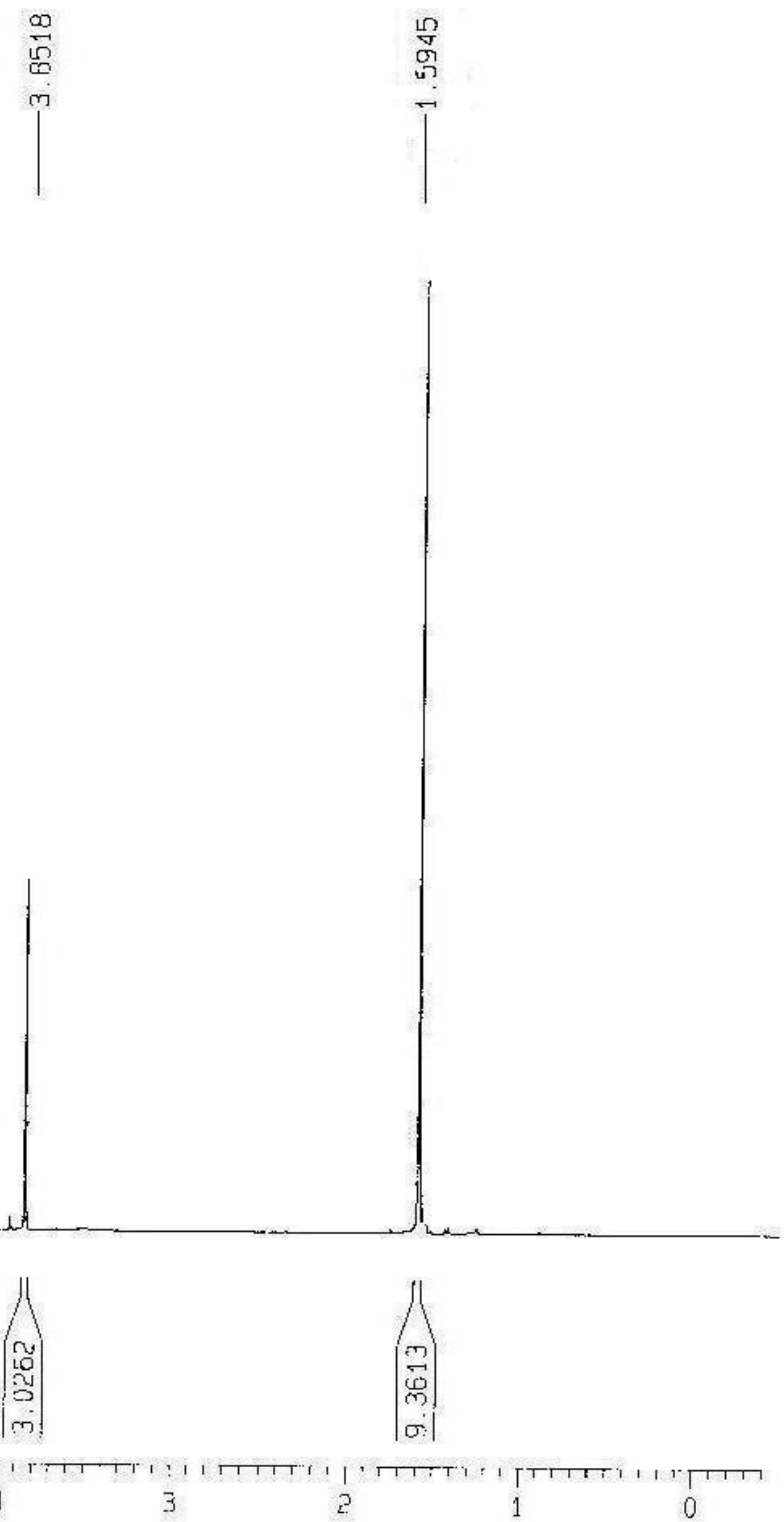

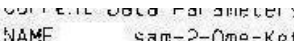

EXPNO

כROCNO

F2 - Acqujsition Parameterइ

$\begin{array}{lr}\text { Jate - } & 20060311 \\ \text { Time } & 12.28\end{array}$

INSTRUM Spect

PROE 1 - $5 \mathrm{~mm}$ BSO BB-1H

PULFROG $\quad 2930$

TID 2930

SOLVENT COCI

VS 15

5WH $5995.204 \mathrm{HI}$

=IDAES $\quad 0.36591 \mathrm{HI}$

$4 Q \quad 1.3654755$

$\begin{array}{cc}\text { AQ } & 1.36647565 \text { Ser. } \\ \text { PG } & 574.7\end{array}$

JW $\quad 83.400$ usec

JE $\quad 5.50$ usec

1.50000000

$====-==$ CHANEL if

$n===-+=$ CHANNEL II $==-===$
NUC1

$\begin{array}{ll}\text { VuC1 } & 1 \mathrm{H} \\ 21 & 10.10 \mathrm{v5ec}\end{array}$

$\quad 3.00 \mathrm{~dB}$

$=2$ - Processing parameters

$\begin{array}{cc}\text { s1 } & 16384 \\ \text { SF } & 409.1300095 \\ \text { Mitz }\end{array}$

WDW $\quad$ EM

$5 S B \quad 0$

$\begin{array}{cc}-B & 0.10 \mathrm{~Hz} \\ \mathrm{~GB} & 0\end{array}$

10 Nup potot oraters

10 NMR plot parameters

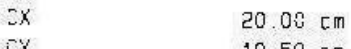

$+1 \mathrm{p} \quad 10.50 \mathrm{~cm}$

$\begin{array}{ll}1 & 10.500 \mathrm{ppm}\end{array}$

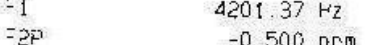

$=2 \quad-200.07 \mathrm{~Hz}$

$\begin{array}{lr}\text { IPNCM } & 0.55000 \mathrm{ppm} / \mathrm{zm} \\ 4 \mathrm{CCM} & 220.0715 ? \mathrm{~Hz} / \mathrm{cm}\end{array}$ 
i. 2 spectriur or

हू

Current Data Parameters YAME Sam TH.2. GMe ket ExPNO

$=2-4$ - 4[quisjtion Parameter

Jatt 20060311

INSTRUM

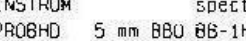

JULPFOS 290930

TD

SOLVENT

NS

$21525.629 \mathrm{~Hz}$

HORES $\quad 0.393387 \mathrm{~Hz}$

20 1.3042164 sec

P. 3649.1

$\begin{array}{lr}\text { DW } & 19.900 \text { usec } \\ \text { DE } & 5.50 \text { usec }\end{array}$

TE 1100000

d11 $\quad 0.03000000$ sec

ग12 $0.00002000 \mathrm{sec}$

$======$ CHANWEL $f t=== \pm+n=0$

$\begin{array}{ll}\text { NUCC } & 13 \mathrm{C} \\ 31 & 10.80 \text { usec }\end{array}$

$\begin{array}{lr}31 & 10.80 \text { use } \\ \text { गL1 } & 7.00 \mathrm{dE}\end{array}$

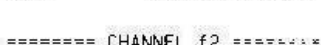

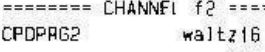

NUEC2 1 IH

90.00 usec
$\mathrm{PCD} 2$

PL2 3.00 ob

PL13

SF $\mathrm{D2} \quad 400.1319000 \mathrm{MH}$

-2 - Processing parameters

51 9racessing parameters 32768

SF $\quad 100.6127729 \mathrm{MHz}$

WDW

5S6

$\mathrm{B}$
$\mathrm{BB}$
$\mathrm{yC}$

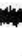

(1)

200

175

125

100

50

25
CX NMA p]at barameters

$20.00 \mathrm{rm}$
$10.00=\mathrm{m}$ 230.000 DD -10.000 . 00 $-1006.13 \mathrm{~Hz}$

$12.0020002 \mathrm{~m} / \mathrm{c}$ $1207.3531 \mathrm{~s} \mathrm{~Hz} / \mathrm{cm}$ 
$H$ spectrumi of

\section{言}
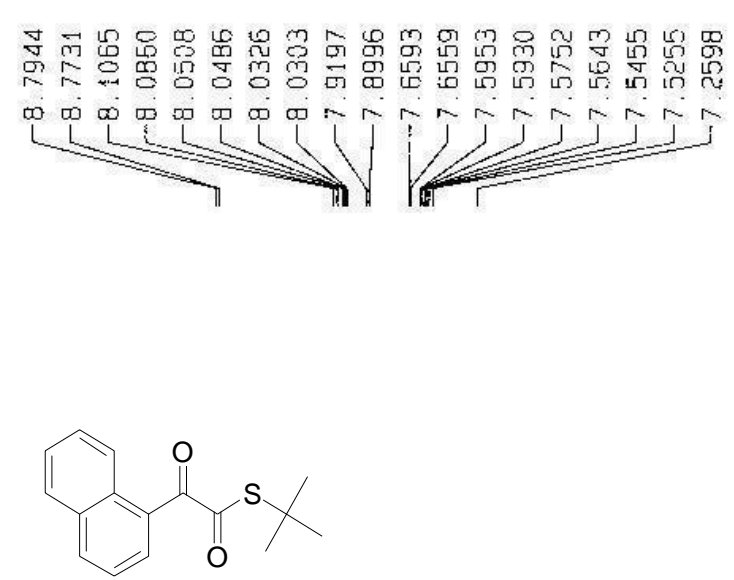

21'
Zur.ment Data Parameters

VAME

EROCNO

F2 - ACquisition Parameters

Date_. 20060426

Tim:- $\quad 1946$

INS? HUM SFect

गROBHD $5 \mathrm{~mm}$ BEO $\mathrm{BB}-1 \mathrm{H}$

בULPROG $\quad 7 \mathrm{~g} 30$

ID $\quad 16384$

NS

SWH $\quad 5995$ 20 $204 \mathrm{~Hz}$

FIDRES $\quad 0.365919 \mathrm{~Hz}$

AQ $\quad 1.3664756 \mathrm{sec}$

PG 287.4

JE

TE $3000 \mathrm{~K}$

$x+150000000 \mathrm{sec}$

$====-+-$ CHANNEL $f 1======$

$\begin{array}{lll}\text { YUC1 } & 1 \mathrm{H} \\ 01 & 10.10 \text { user }\end{array}$

PI 1 a

SF 0144001326002 Mitz

ت2. Processing parameter

५1

SF $\quad 400.1300095 \mathrm{MHZ}$

$\begin{array}{lll}\mathrm{NDW} & \mathrm{EM} \\ \Xi S \mathrm{~B} & \end{array}$

$-B \quad 0.16 \mathrm{~Hz}$

$\mathrm{GB}$
$\mathrm{FC}$

0

10 NMH plot páraneters

cx $2000 \mathrm{~cm}$

Cy $\quad 10.50 \mathrm{~cm}$

$=1 \mathrm{P} \quad 10.500 \mathrm{pFm}$

$\begin{array}{ll}=1 & 43.53 .37 \mathrm{HL} \\ =35 & -3.500 \mathrm{HLm}\end{array}$

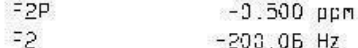

JPMCM $\quad 0.55000 \mathrm{DDT} \mathrm{T/Cm}$

$\rightarrow Z 2 \mathrm{CMM} \quad 220.07150 \mathrm{~Hz} / \mathrm{CI}$

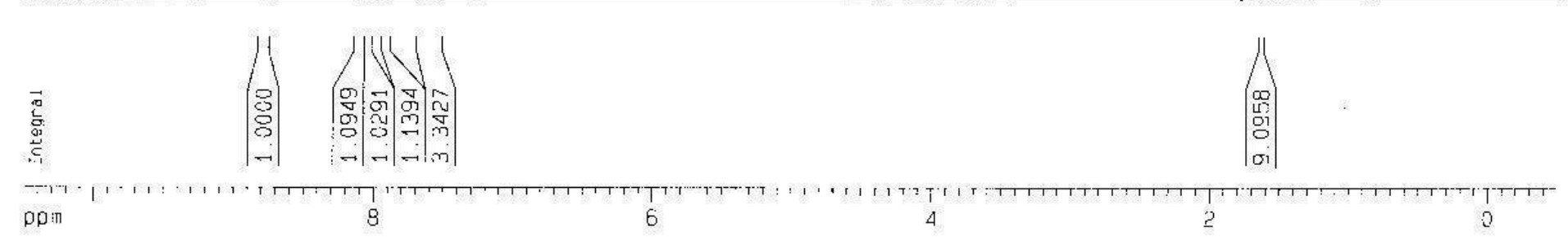


C13 spectrum [f

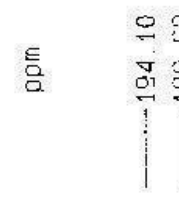

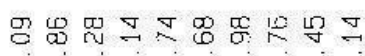

Current Cata Parmemeters

NAME

EXPNO
PROCNO

F2 - Acquisition Fanamet.ers

Wate. P006032

1)

INSTGUM

Epece

TO $\quad 65536$

sol.

5HH $25155.629 \mathrm{~Hz}$

=IDRES $\quad 0.363397 \mathrm{~Hz}$

AQ $\quad \therefore .3042164 \mathrm{se}$

JW $\quad 19900$ user

IL

D: 1.2000000 5te.

d11 $\quad$ c.03060500 sec

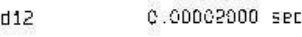

$======0$ CHANNE: $+11 \ldots== \pm=$

$\begin{array}{lll}\text { NuCs } & 130 \\ 11 & 10.8 C \\ \mathrm{Jsec}\end{array}$

$\begin{array}{lr}\text { Pl } 1 & 1.00 \mathrm{~dB} \\ \text { SFI1 } & 100.6242995 \mathrm{MHJ}\end{array}$

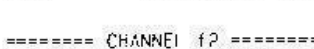

CPDPAGẼ

VULC 11

$\exists \bar{c} \quad 90.00$ use

I 12 21.93 d8

PL13
SF02

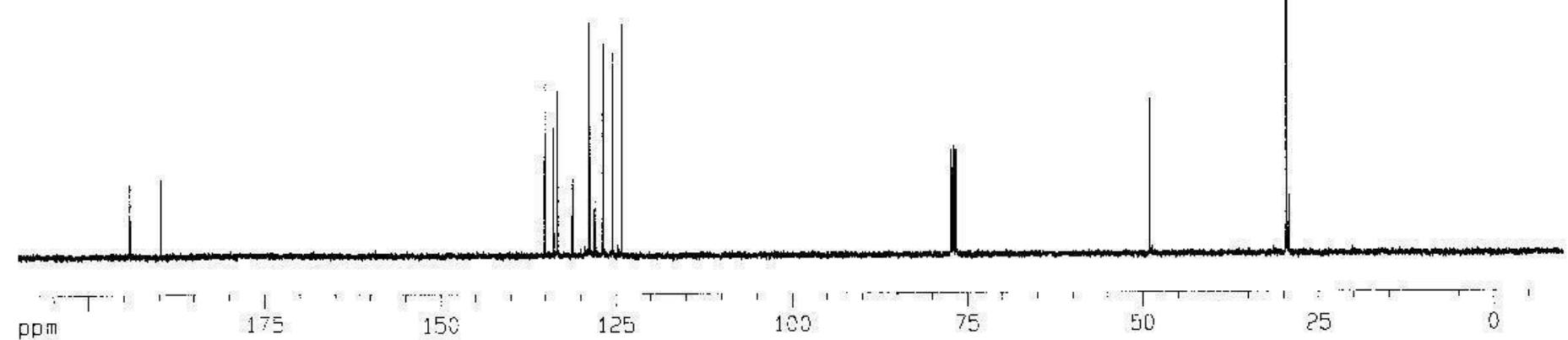

- Procesaing paramcter

SI 327 E

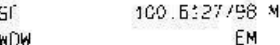

WDW
$\subseteq S B$

38
$\therefore B$

10. NMP plat parameters

Ex $2000 \mathrm{~cm}$

$\begin{array}{lll}\mathrm{cr} & 1002 \mathrm{~cm} \\ =1 \mathrm{P} & 210.000 \mathrm{~cm}\end{array}$

$\because 1 \quad$ 21122 $69 \mathrm{~Hz}$

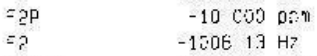

गतMCM 11. $00000 \mathrm{p}=\mathrm{m} / \mathrm{Cm}$ 
H spectrum of

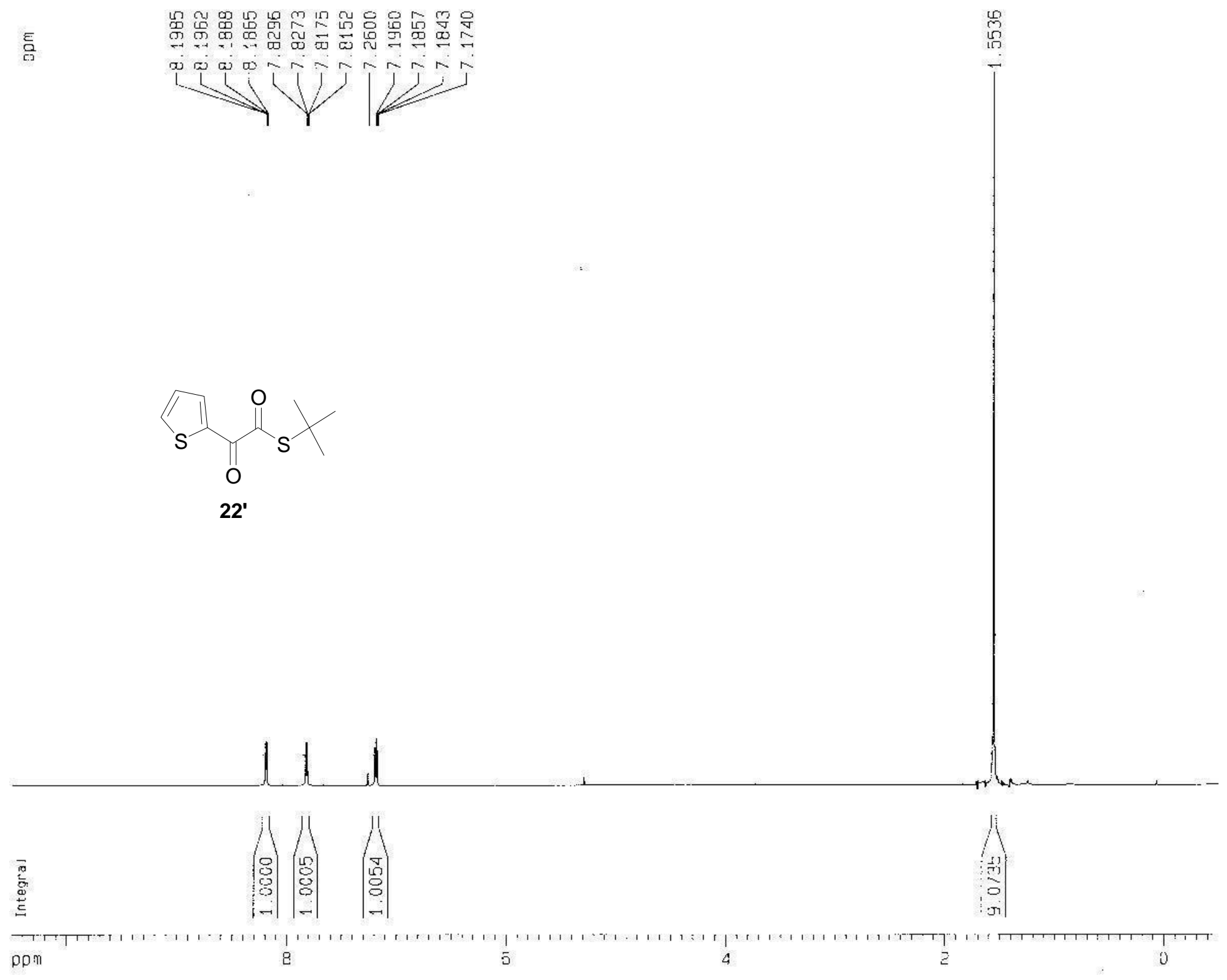

VAM:

EXPND

Eam-Eh10-Ket

$=2$.. Acquisition Parameters

vate_ 200604?

1 1ाए 17.43

INSTRUM SFErt

RISEHT 5 mI BSO $\mathrm{BE}-1$

UU_PROG zg30

TD

30. VEN: CDL13

vS

$\begin{array}{cc}7 \mathrm{~S} & 0 \\ 3 \times 4 & 5995.204 \mathrm{~Hz}\end{array}$

=IJRES $\quad 3.355918 \mathrm{~Hz}$

$40 \quad 1.306</ 56$ sec

JE

21

$: 01.6$

b. 50 use

$300.0 \mathrm{~K}$

1.50060000 sec

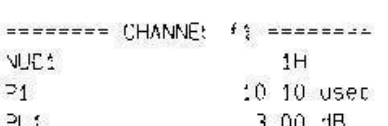

ग.: $\quad 3.00 \mathrm{JB}$

SF51 $4001326008 \mathrm{MHZ}$

$=2$ Processing oarameters

31 P $33 \mathrm{BA}$

SF $400.1300095 \mathrm{MHz}$

HDW

$\begin{array}{ll}-B & 0.10 \mathrm{~Hz}\end{array}$

莇

10 NMP plot parameters

$\begin{array}{ll}2 x & 20.00 \leq m \\ 2 y & 1500=m\end{array}$

$\begin{array}{lrl}24 & 15.00 \leq m \\ =10 & 10.500 & 02 \pi\end{array}$

$=1 \quad 4201.37 \mathrm{~Hz}$

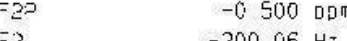

PPMCM

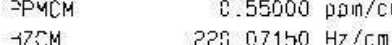


¿13 spectrum of

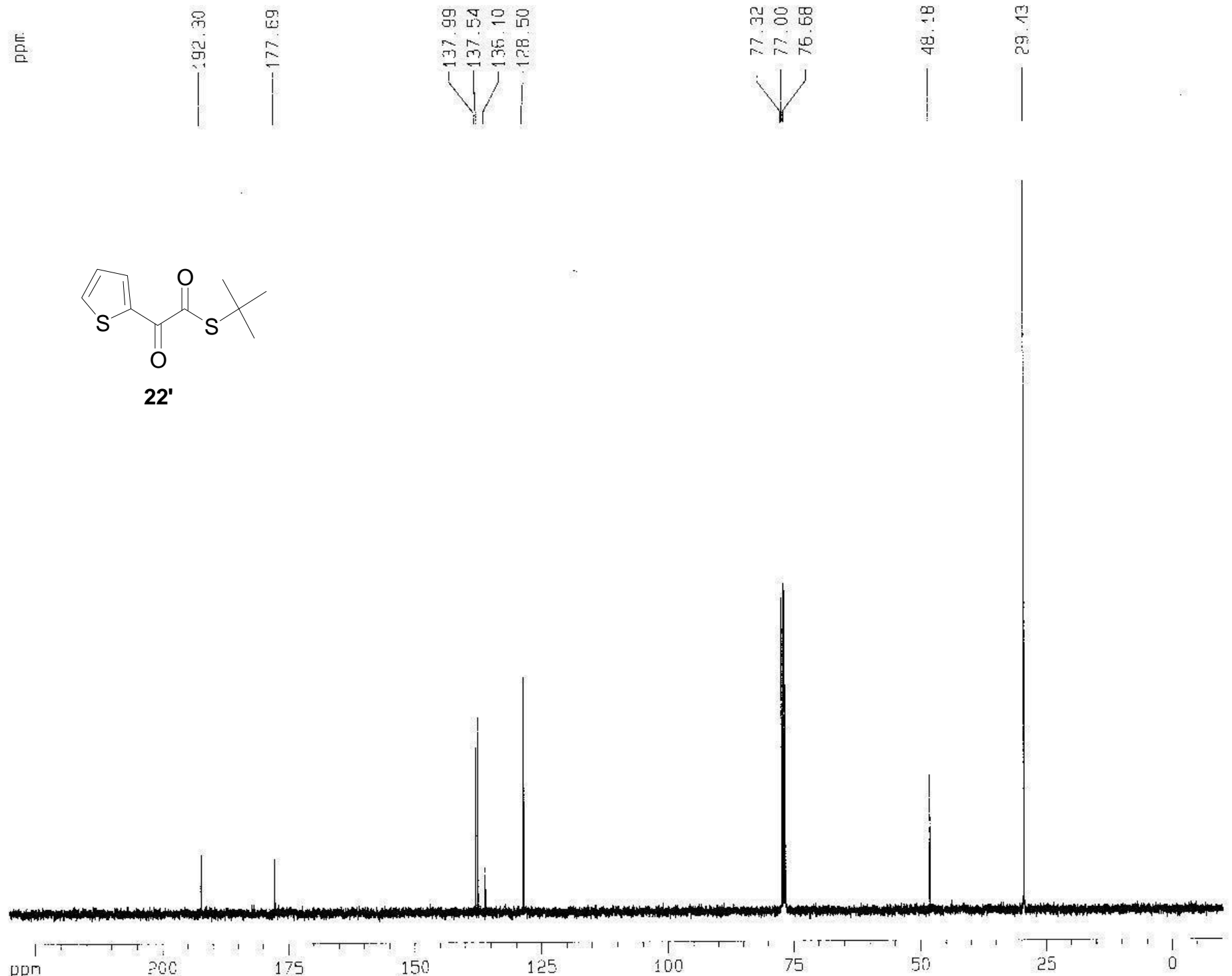

urrent Data Parancters

NAME sam

EXOCNO

5- Acqujsit jon Fananeters

$11 \mathrm{me}$

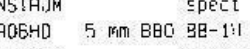

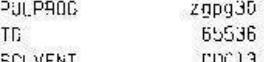

IS
ShiH

Shit 25125 a

c. $383367 \mathrm{~Hz}$

.30421E4 $\mathrm{sec}$

JW 19.900 usec

6.50 usec

$\begin{array}{ll}111 & 0.03000000 \\ 312 & 0.0060 \% 000\end{array}$

$\cdots== \pm=$ CHANNEL $11=-\cdots$.

$\operatorname{suc}_{1} \quad: 0.80$ usec

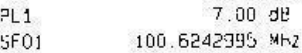

$== \pm===$ CHAHNLLL $+\hat{z} .====$

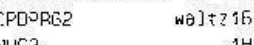

NUC2
PCPD2

$\begin{array}{lll}\text { ग ? } & 3.00 \mathrm{~dB}\end{array}$

ग 125

FFG $\quad 400.1319600 \mathrm{M}-7$

$=$ ? - Procezsura parameters

SI 3276 6

100.612744

${ }^{\mathrm{NOH}} \mathrm{OHB}$

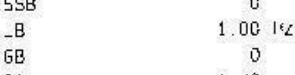

1.10

10. NMMR plot parameiers

$\begin{array}{cc}\mathrm{Ex} & 20.00 \mathrm{~cm} \\ \mathrm{cy} & 12.50 \mathrm{~cm}\end{array}$

$\begin{array}{ll}-1 . & 12.50 \mathrm{~cm}\end{array}$

$=1 \quad 23140.94 \mathrm{~Hz}$

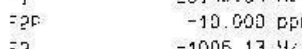

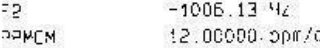

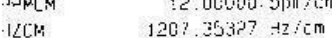


Fi spectrum of

$\rightarrow+1$

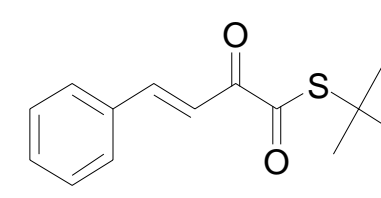

23'

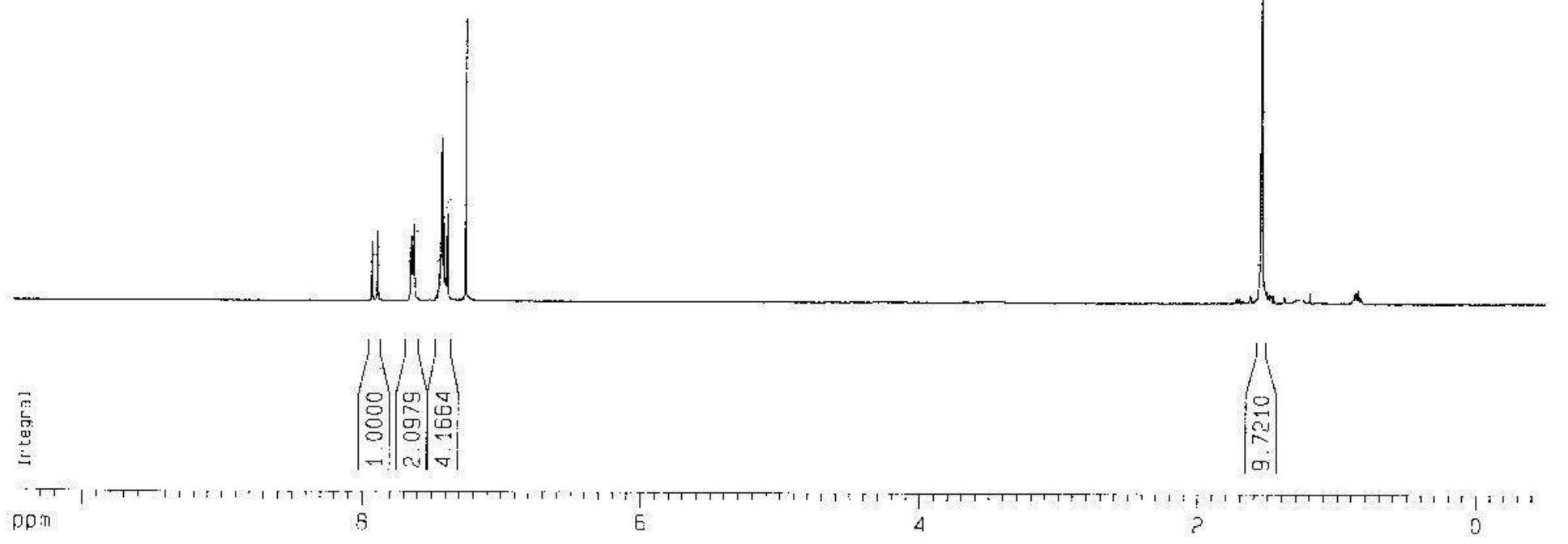

Mivent hata har amecers

MAME 5 am-Cinar - HT

XXNO $-\ln$
1

$\bar{z}-$ Acjulsition Paraneter

Jate_ 20050426

ime $15.3 \mathrm{~B}$

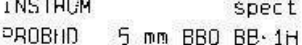

PULPID 5 mII BBO Be. $1 \mathrm{H}$
2030

$\begin{array}{lr}\text { TIL } & 2930 \\ \text { TO } & 16384\end{array}$

SOI.VENT

sivent

DS

5995.204 Hz

:CRES $\quad 0.365918 \mathrm{~Hz}$

AQ $\quad 1.3664756$ se 456.1

83. 400 usec

6. 50 user $30.0 \mathrm{~K}$ 1. $50000000 \mathrm{sec}$

01

CHANNEL $f 1=======$ $\begin{array}{ll}\text { NUC1 } & 1 \mathrm{H} \\ \mathrm{P} & 10.10 \text { use }\end{array}$ $\begin{array}{lc}\mathrm{Pi} & 10.10 \mathrm{usec} \\ \mathrm{il} & 300 \mathrm{de}\end{array}$

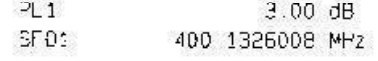

$-a$ - Procossing ganameters SI 16384 SF $\quad 400.1300095 \mathrm{MHz}$ W.JW
SSB $\begin{array}{ll}-\mathrm{B} & 0.10 \mathrm{~Hz}\end{array}$ $\begin{array}{ll}\mathrm{GB} & 0 \\ \mathrm{IC} & 100\end{array}$

10 NMR plot parameters

cx 20.00 t.:T CY 20.00 r.m $\begin{array}{lr}2: P & 10.500 \mathrm{opm} \\ -1 & 4201.37 \mathrm{~Hz}\end{array}$ $\begin{array}{rl}-1 & 4201.37 \mathrm{~Hz}\end{array}$ $=2 \quad-30006 \mathrm{kz}$ SFMCM $\quad 0.55000 \mathrm{pPm} / \mathrm{Cm}$ 
ह.

o

$\mathrm{s}$

O

23'

C13 spectrum of

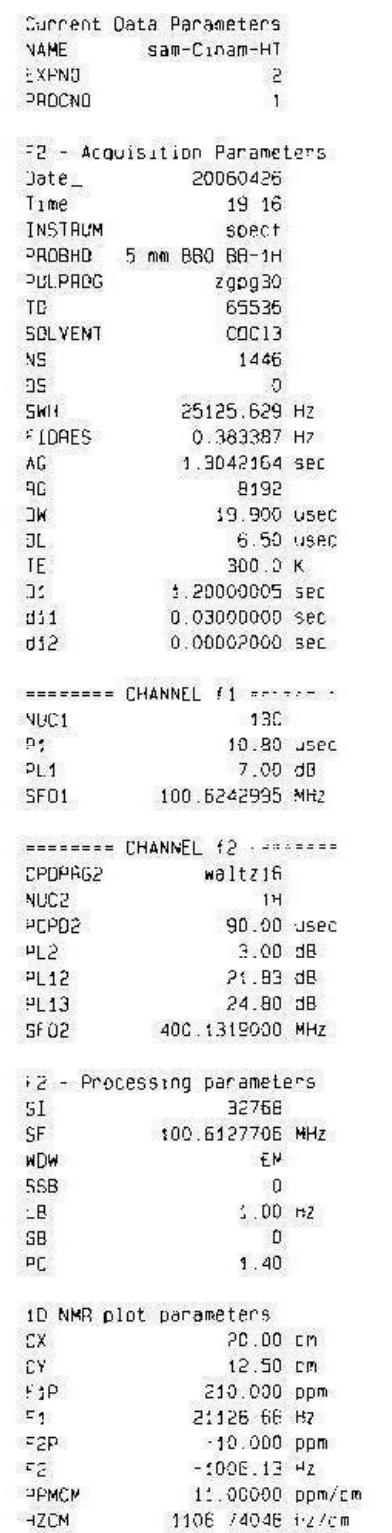


$H$ spectrum of

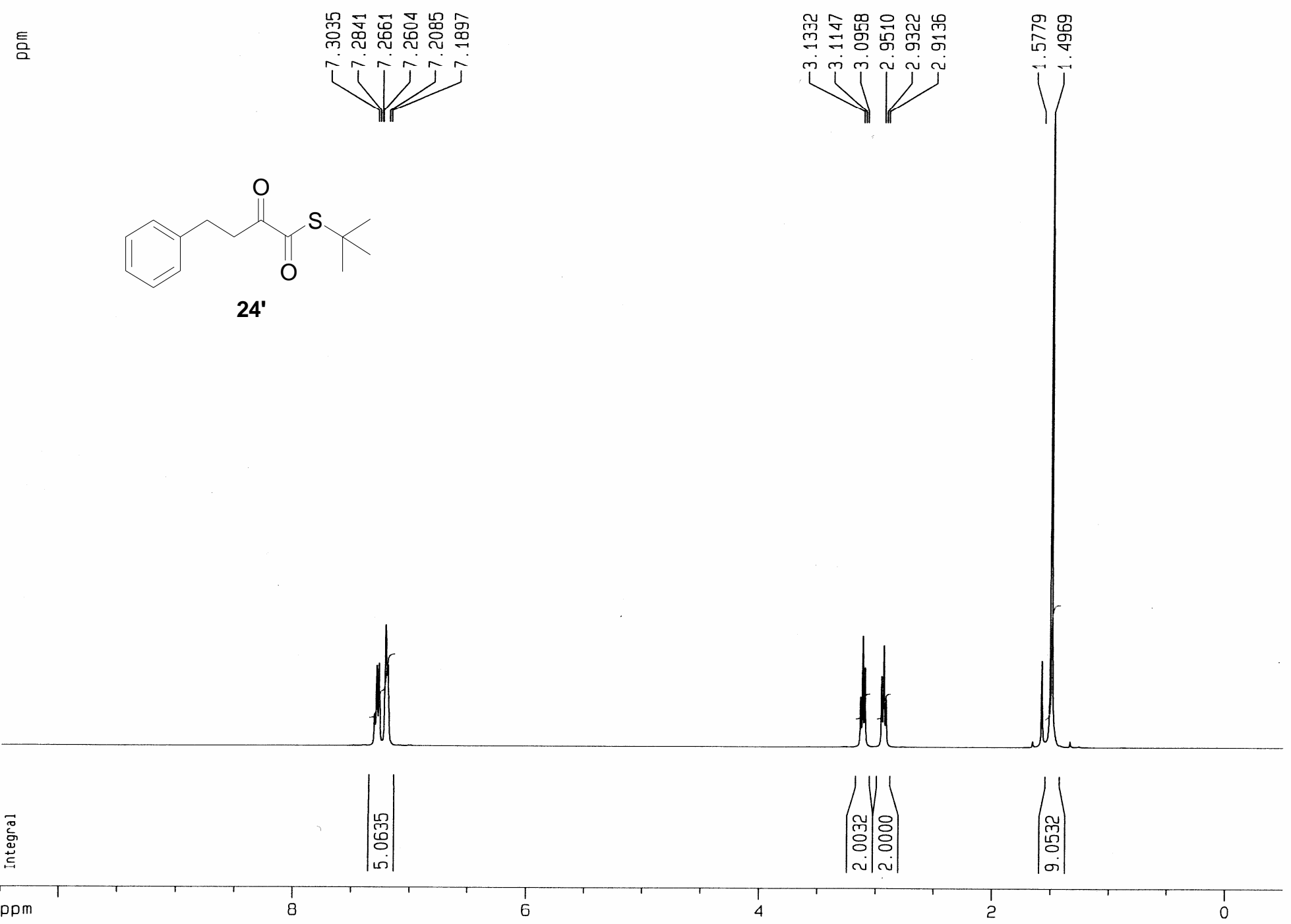

Current Data Parameters NAME

EXPNO

F2 - Acquisition Parameters

Date 20060930

Time 12.29

INSTRUM spect PROBHD $5 \mathrm{~mm}$ BBO BB-1H $\begin{array}{lr}\text { PULPROG } & 2930 \\ \text { TD } & 16384\end{array}$

$\operatorname{coc} 13$

NS

SWH $\quad 00$

$\begin{array}{ll}\text { FWH } & 5995.204 \mathrm{~Hz} \\ \text { FIDRES } & 0.365918 \mathrm{~Hz}\end{array}$

$A Q \quad 1.3664756 \mathrm{sec}$

$\begin{array}{lc}\text { RG } & 1.3664756 \mathrm{sec} \\ \text { OW } & 203.2\end{array}$

DW 83.400 usec

$\begin{array}{cc}\text { DE } & 6.50 \text { use } \\ \text { TE } & 298.1 \mathrm{~K}\end{array}$

TE $\quad 298.1 \mathrm{~K}$

$\begin{array}{ll}\text { D1 } & 1.50000000 \mathrm{sec} \\ \text { MCREST } & 0.00000000 \mathrm{sec}\end{array}$

MCWRK $\quad 0.01500000 \mathrm{sec}$

$=======$ CHANNEL $f 1$ = $======$

$\begin{array}{ll}\text { NUC1 } & 1 \mathrm{H} \\ \mathrm{P} 1 & 10.30 \mathrm{use}\end{array}$

$\begin{array}{lr}\text { Pl } & 10.30 \text { usec } \\ \text { PL1 } & 2.00 \mathrm{~dB} \\ \text { SFO1 } & 400.1326008 \mathrm{MHz}\end{array}$

Fa - Processing poraneters

F2 - Processing parameters
SI

$\begin{array}{lc}\text { SI } & 16384 \\ \text { SF } & 400.1300099 \mathrm{MHz}\end{array}$

WDW

LSB

$1.00 \mathrm{~Hz}$

10 NMR plot parameters

CX $20.00 \mathrm{~cm}$

$\begin{array}{ll}\mathrm{CY} & 20.00 \mathrm{~cm} \\ \mathrm{FF} & 20.00 \mathrm{~cm}\end{array}$

F1P $\quad 10.500 \mathrm{ppm}$

F1 $\quad 4201.37 \mathrm{~Hz}$

$\begin{array}{ll}\mathrm{F} 2 \mathrm{P} & -10.500 \mathrm{DPm} \\ \mathrm{F2} & -0.500 \mathrm{ppm}\end{array}$

$\begin{array}{ll}\text { F2 } & -200.06 \mathrm{~Hz} \\ \text { PPMCM } & 0.55000 \mathrm{pom} / \mathrm{cm}\end{array}$

$\begin{array}{ll}\mathrm{HZCM} & 0.55000 \mathrm{ppm} / \mathrm{cm} \\ & 220.07150 \mathrm{~Hz} / \mathrm{cm}\end{array}$ 
C13 spectrum of
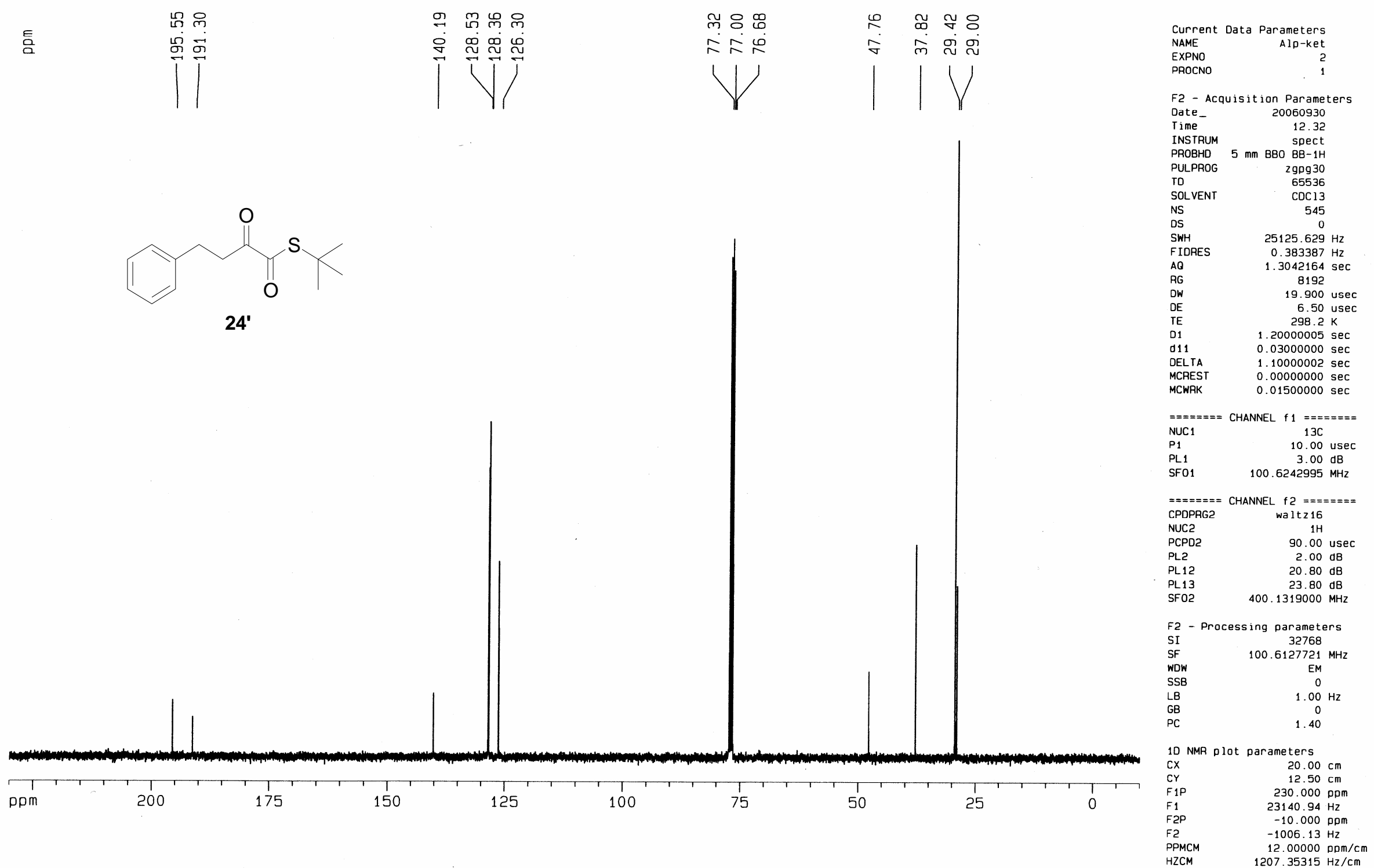
Racemic (97/3, AD-H) (8a)

$\mathrm{OH}$

$\mathrm{S}$

O

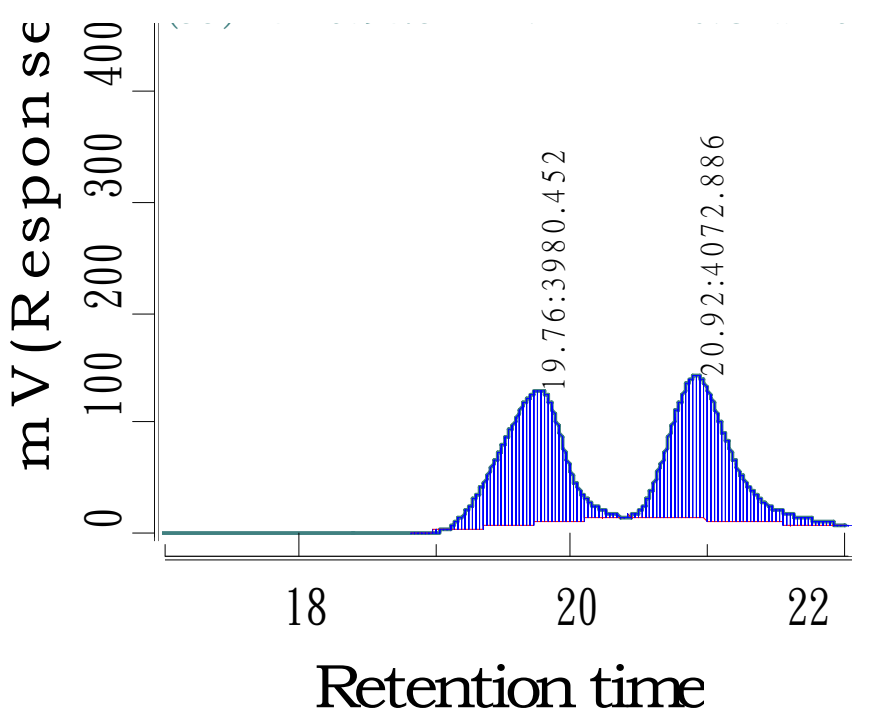

$\begin{array}{rrrr}\text { 滯留時間 } & \text { 面積 } & \text { 百分比 } & \text { 濃度 } \\ 19.764 & 3980.45 & 49.43 & 0.00 \\ 20.917 & 4072.89 & 50.57 & 0.00\end{array}$

Resolved

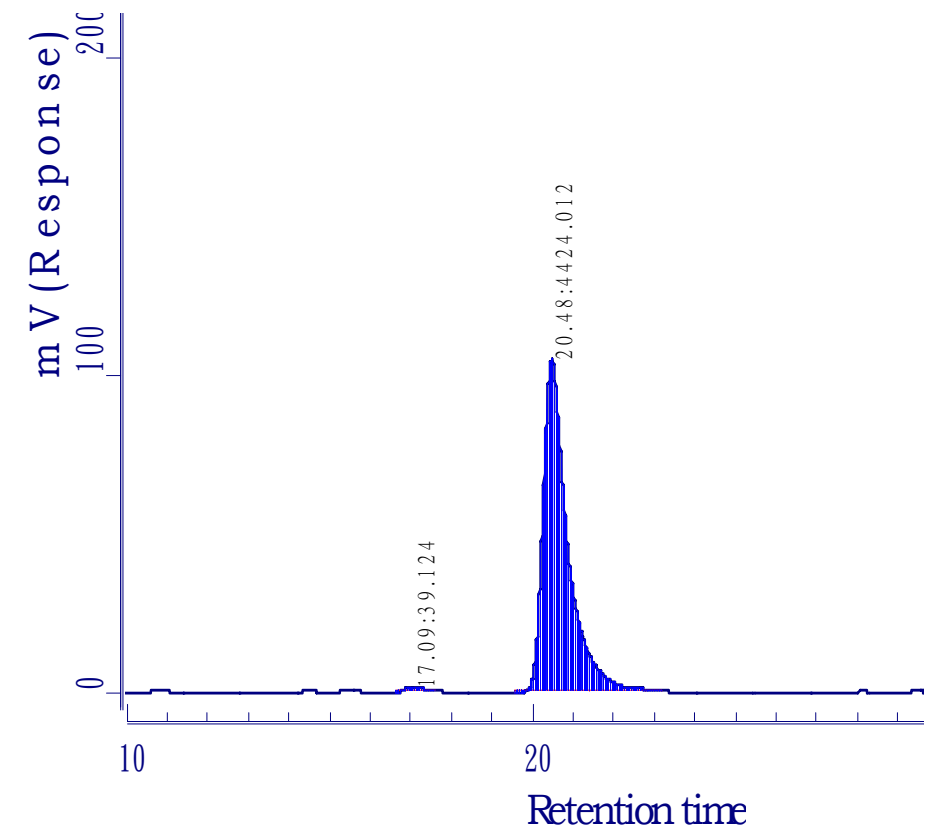

$\begin{array}{rrrrr}\# \text { ～成分峰名稱 } & \text { 滯留時間 } & \text { Area } & \text { 百分比 } & \text { 濃度 } \\ 0 & 17.085 & 39.12 & 0.88 & 0.00 \\ 1 & 20.482 & 4424.01 & 99.12 & 0.00\end{array}$


Racemic (99/1, AD-H) (8b)

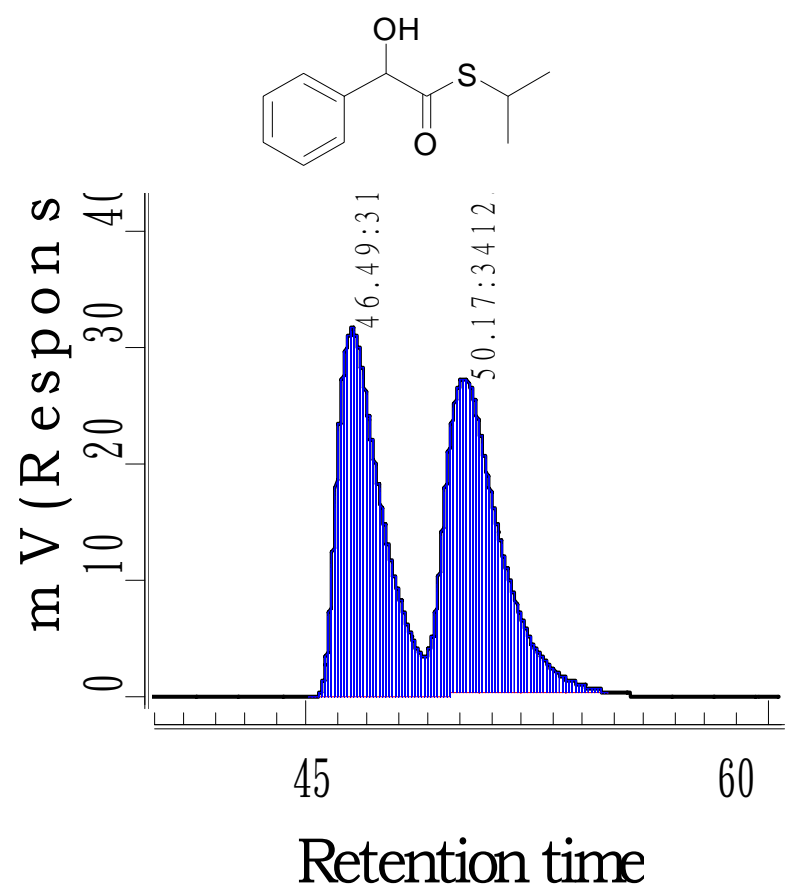

滯留時間

百分比

濃度

0
46.490

50.167
Resolved

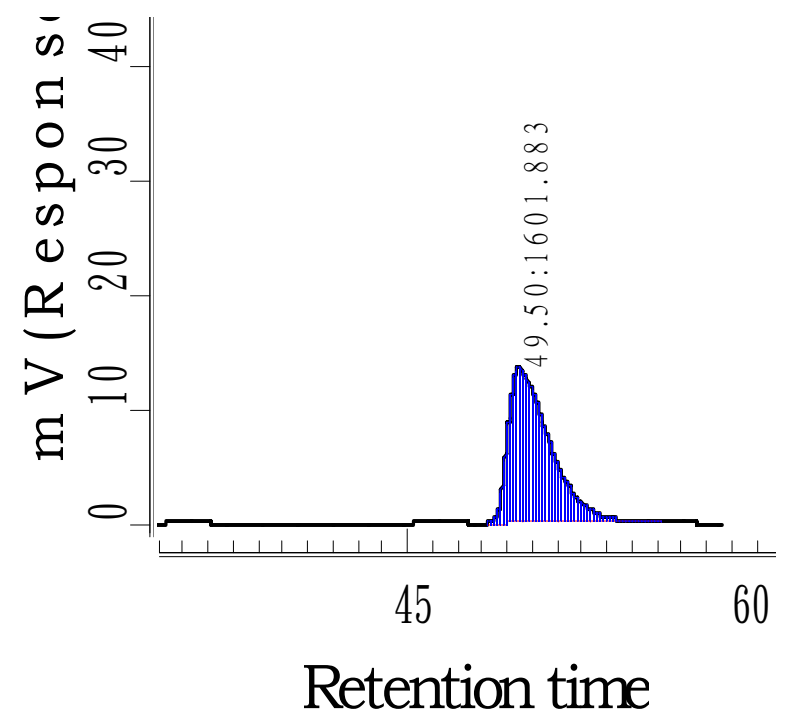

\# 成分峰名稱

滯留時間

百分比 
Racemic (94/6, AD-H) (8c)
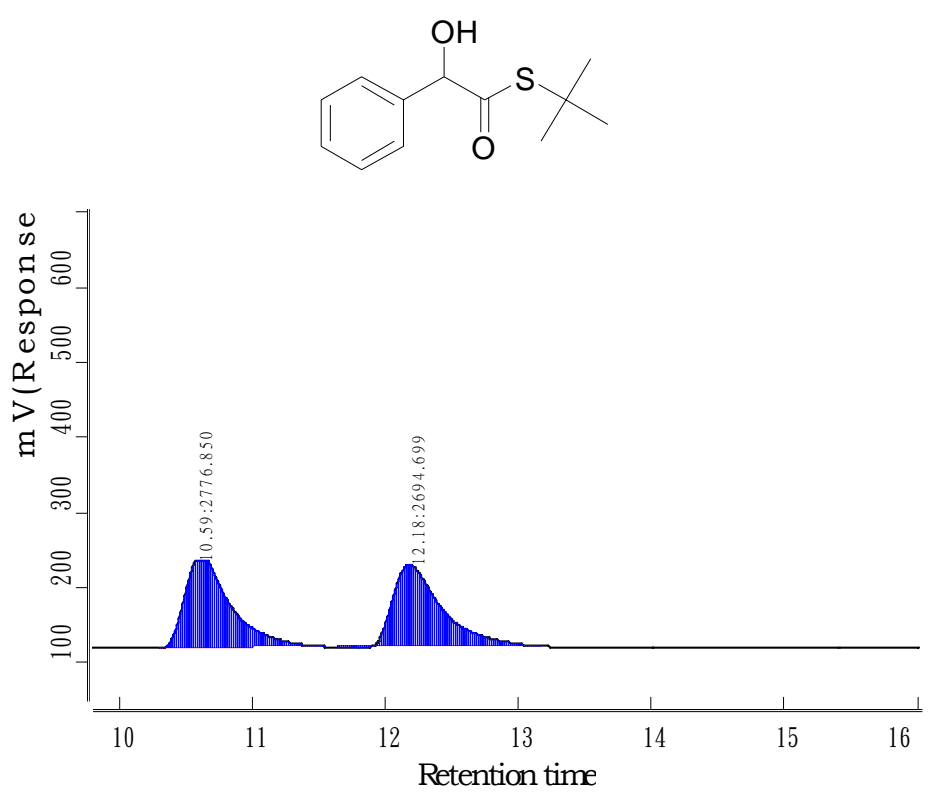

\begin{tabular}{|c|c|c|c|}
\hline \# 成分峰名稱 & 滯留時間 & Area & 百分比 \\
\hline 0 & 10.588 & 2776.85 & 50.75 \\
\hline 1 & 12.184 & 2694.70 & 49.25 \\
\hline
\end{tabular}

Resolved

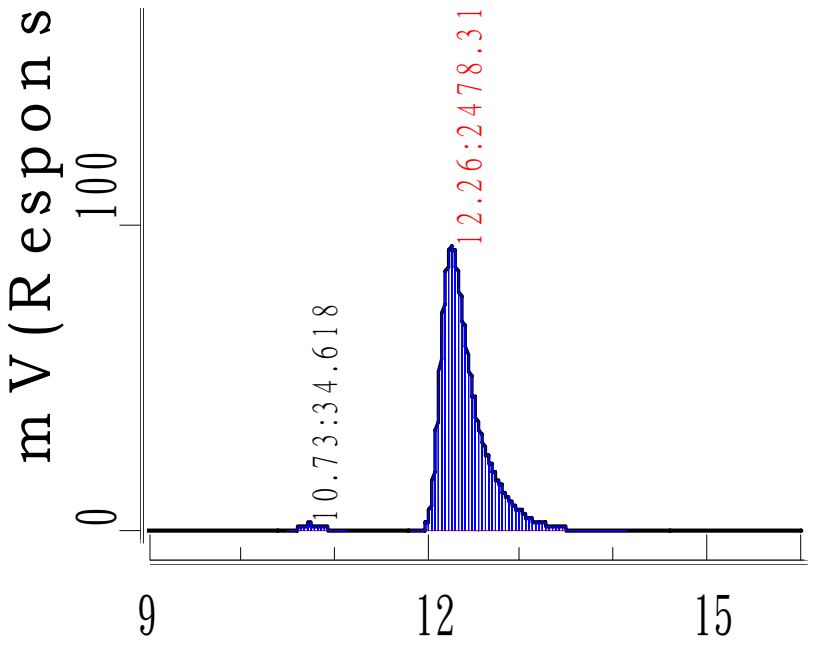

Retention time

$\begin{array}{rrrrr}\# \text { ～成分峰名稱 } & \text { 滞留時間 } & \text { Area } & \text { 百分比 } & \text { 濃度 } \\ 0 & 10.729 & 34.62 & 1.38 & 0.00 \\ 1 & 12.262 & 2478.32 & 98.62 & 0.00\end{array}$


Racemic (94/6, AD-H) (8e)

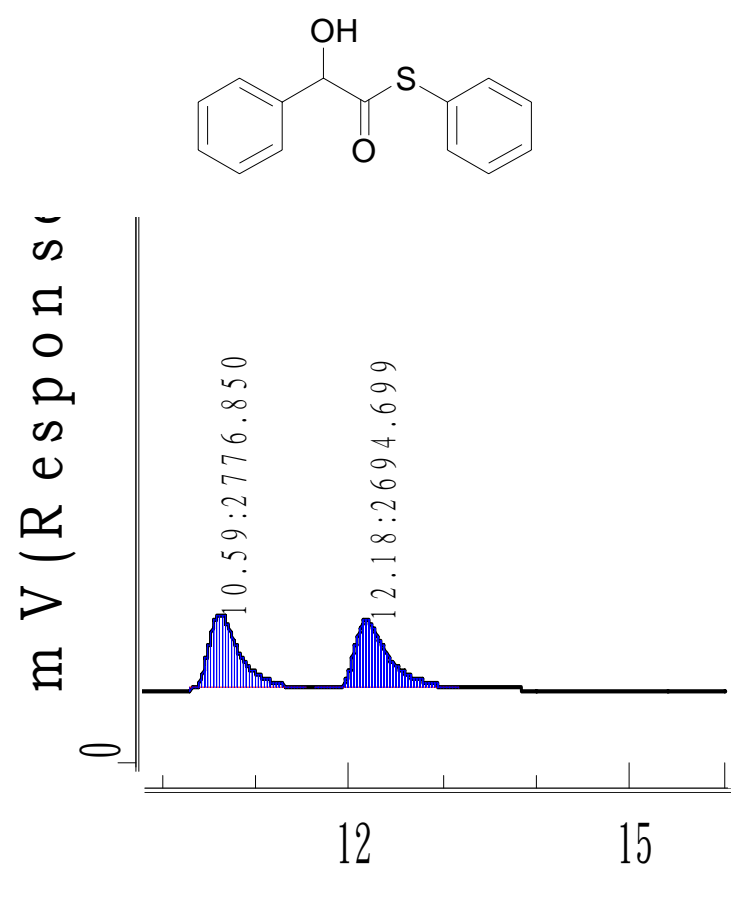

Retention time
Resolved

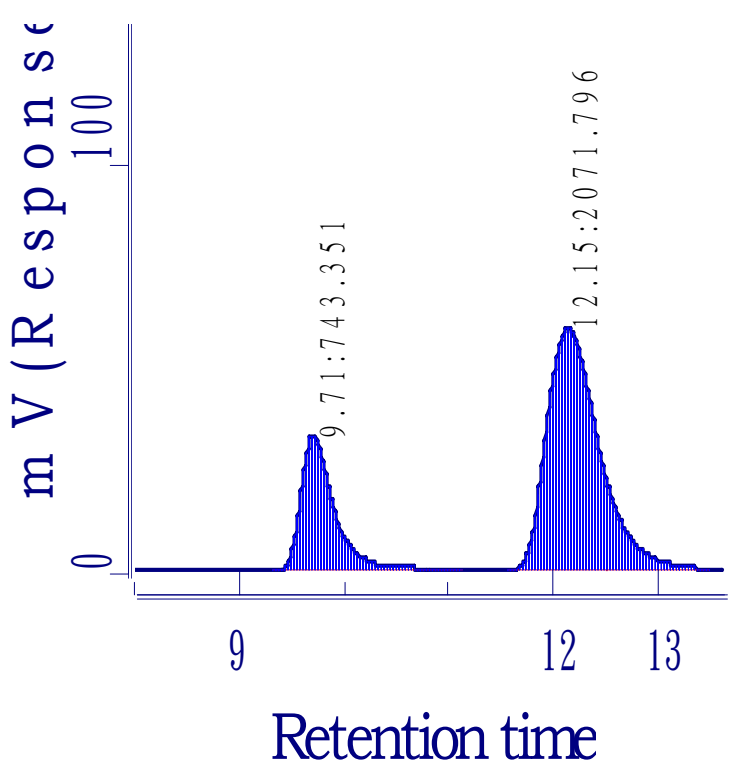

\# 成分峰名稱

$\begin{array}{rrrr}\text { 滞留時間 } & \text { Area } & \text { 百分比 } & \text { 濃度 } \\ 9.709 & 743.35 & 26.41 & 0.00 \\ 12.149 & 2071.80 & 73.59 & 0.00\end{array}$


Racemic (88/12, AD-H) (9)

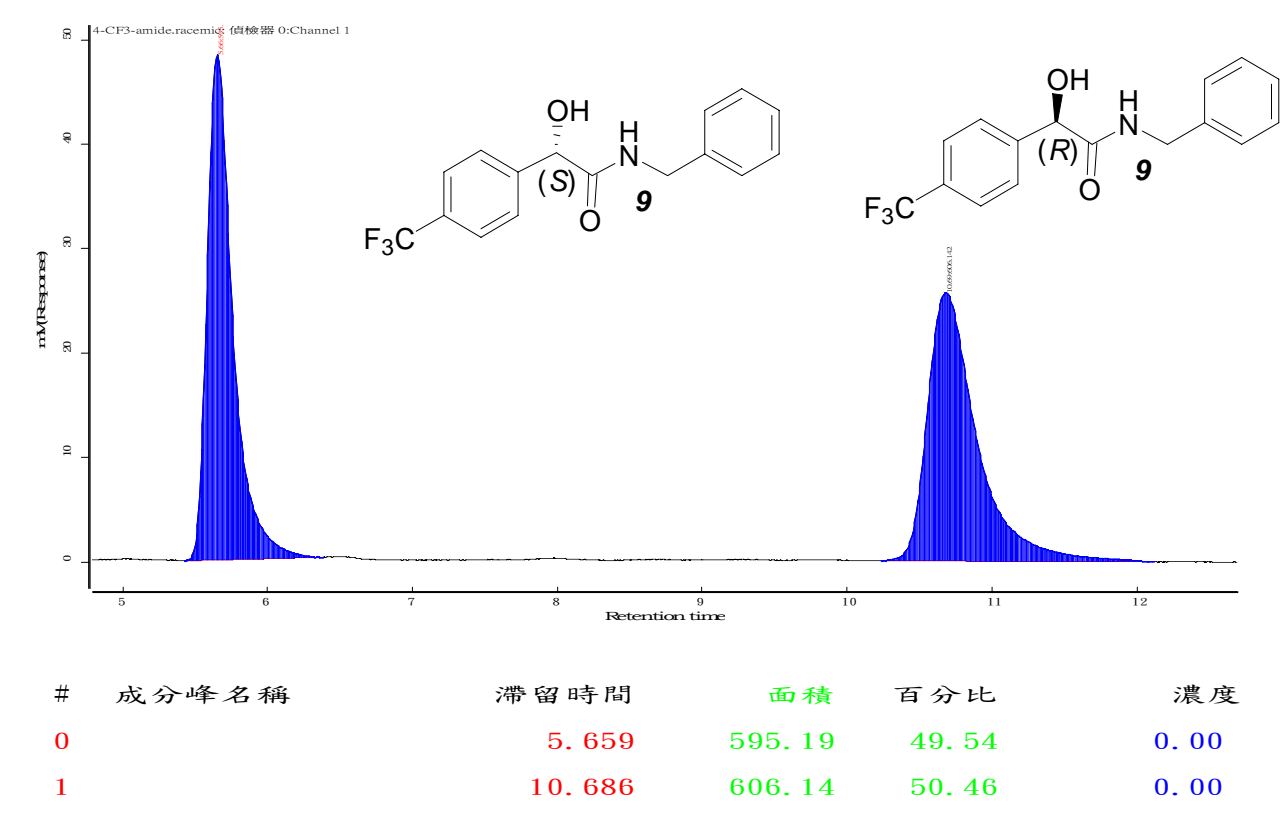

Resolved

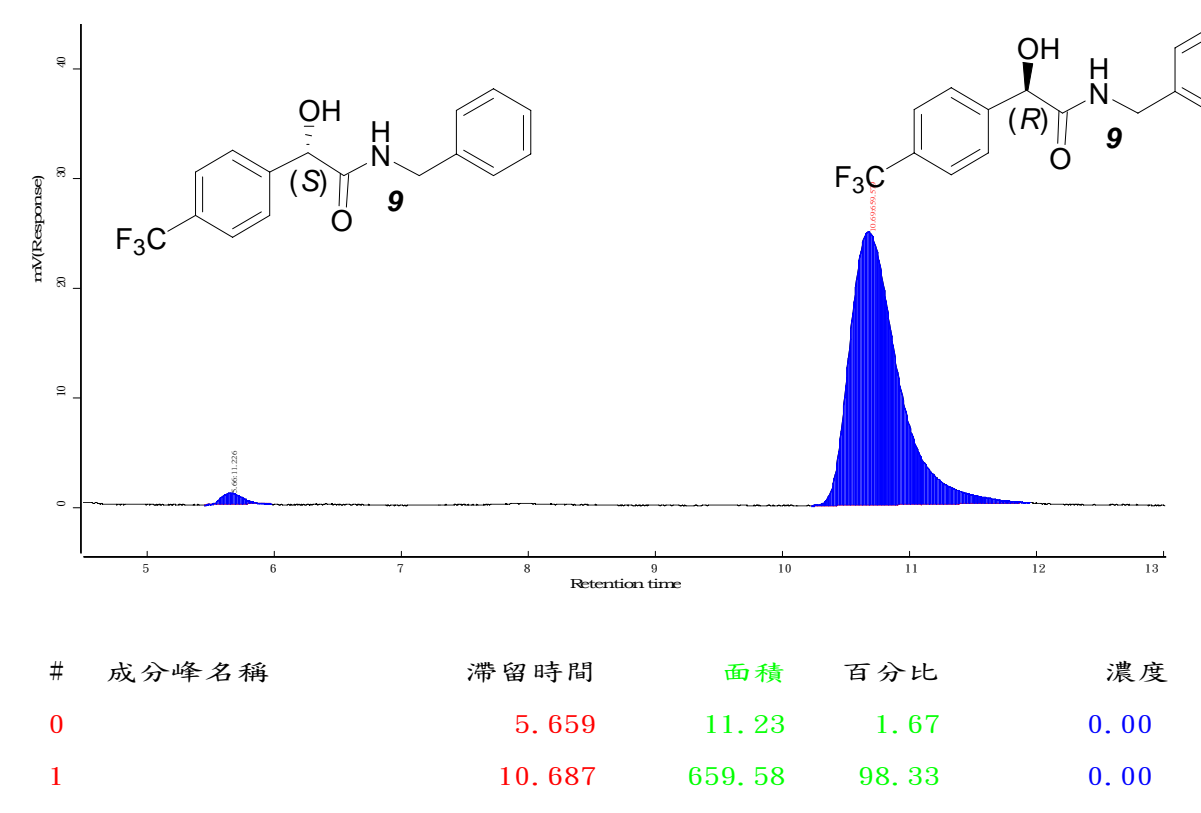




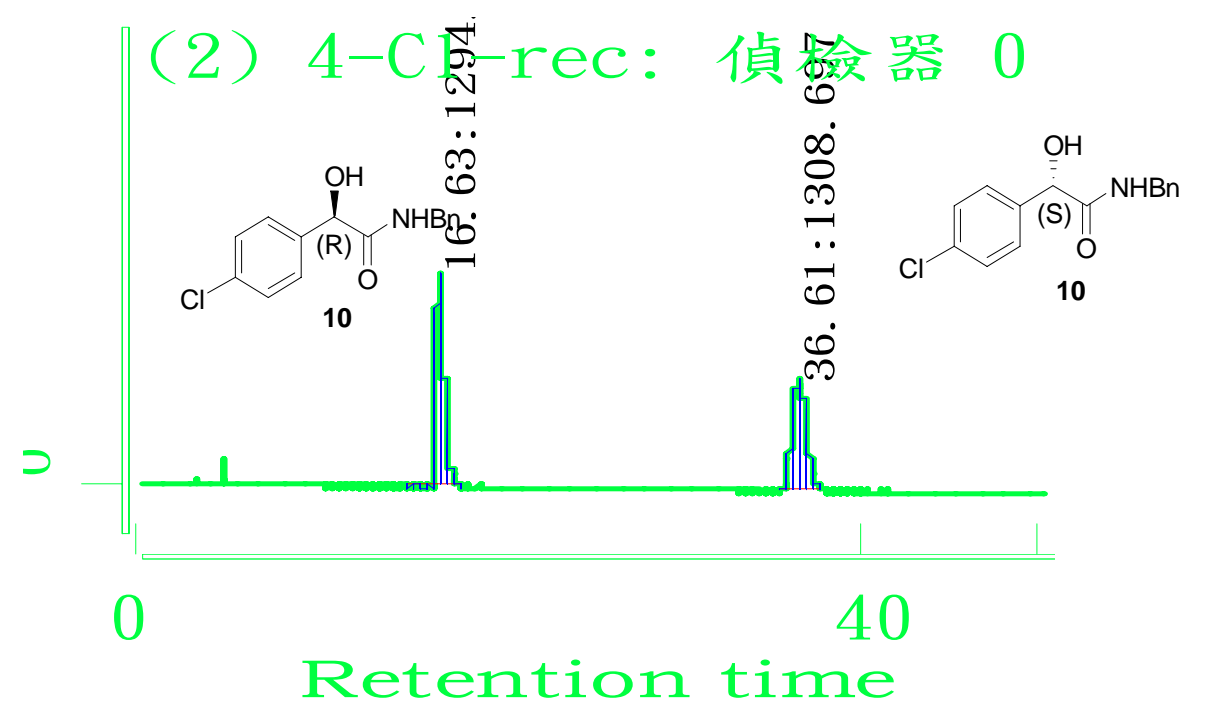

$\begin{array}{rrcc}\text { 滯留時間 } & \text { 面積 } & \text { 百分比 } & \text { 濃度 } \\ 16.634 & 1294.52 & 49.73 & 0.00 \\ 36.607 & 1308.70 & 50.27 & 0.00\end{array}$

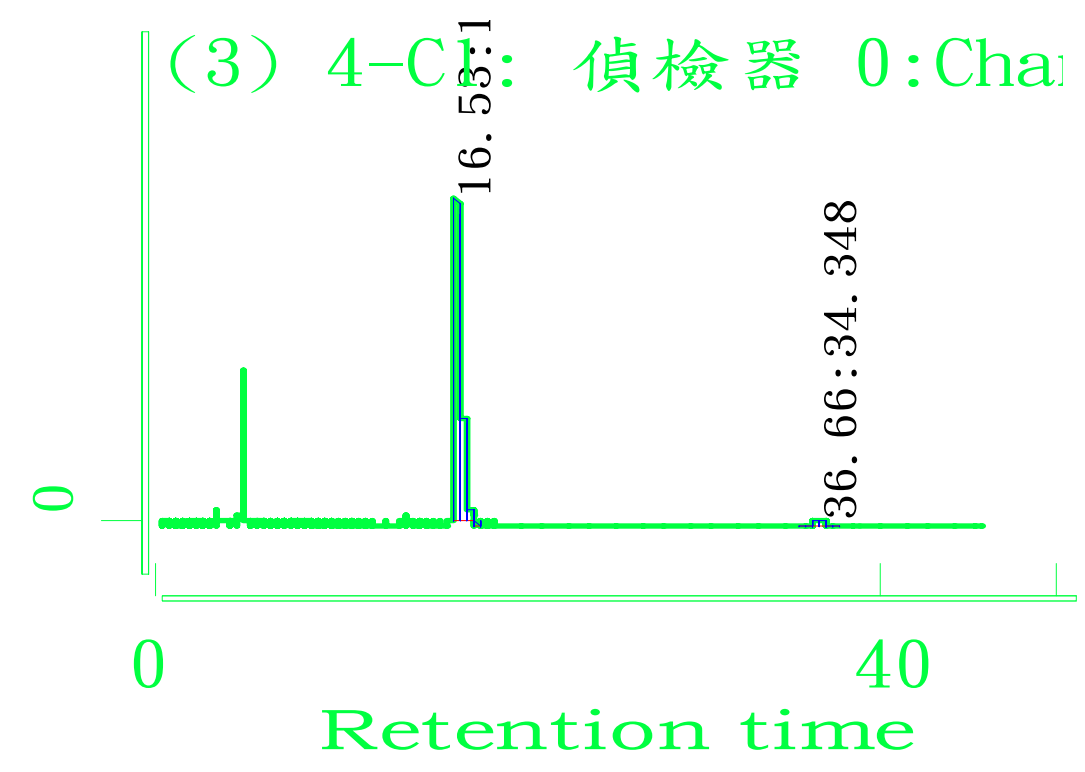

$\begin{array}{rrrr}\text { 滞留時間 } & \text { 面積 } & \text { 百分比 } & \text { 濃度 } \\ 16.529 & 1921.12 & 98.24 & 0.00 \\ 36.658 & 34.35 & 1.76 & 0.00\end{array}$




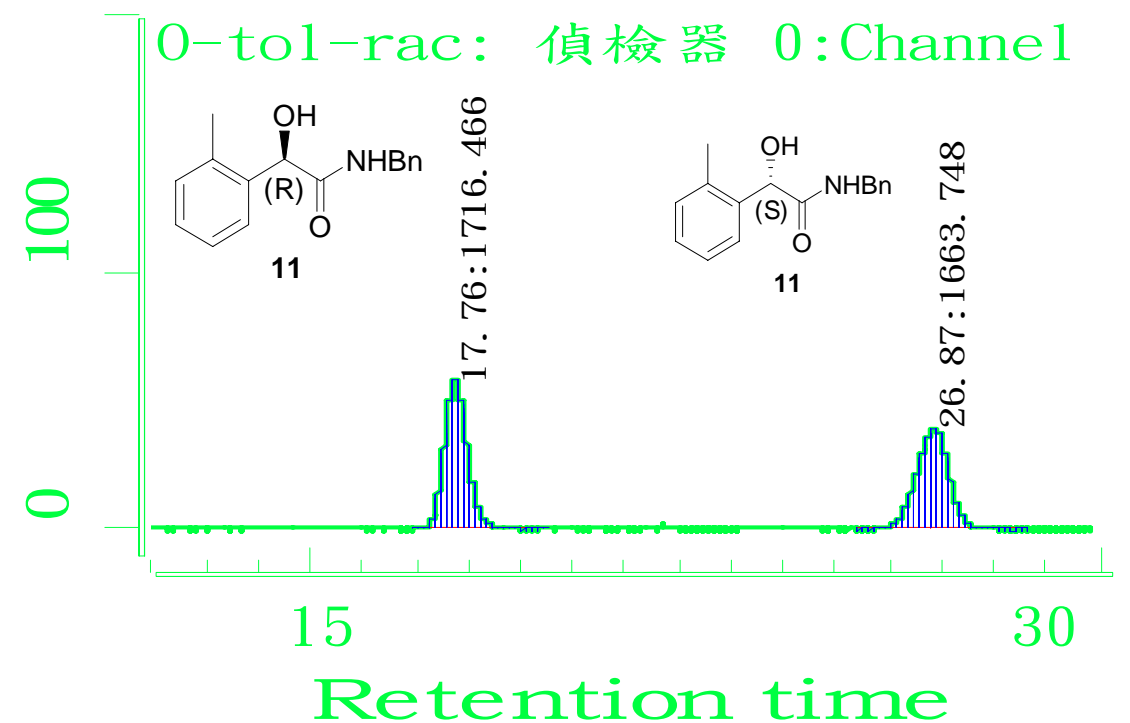

$\begin{array}{rrrr}\text { 滯留時間 } & \text { 面積 } & \text { 百分比 } & \text { 濃度 } \\ 17.764 & 1716.47 & 50.78 & 0.00 \\ 26.867 & 1663.75 & 49.22 & 0.00\end{array}$

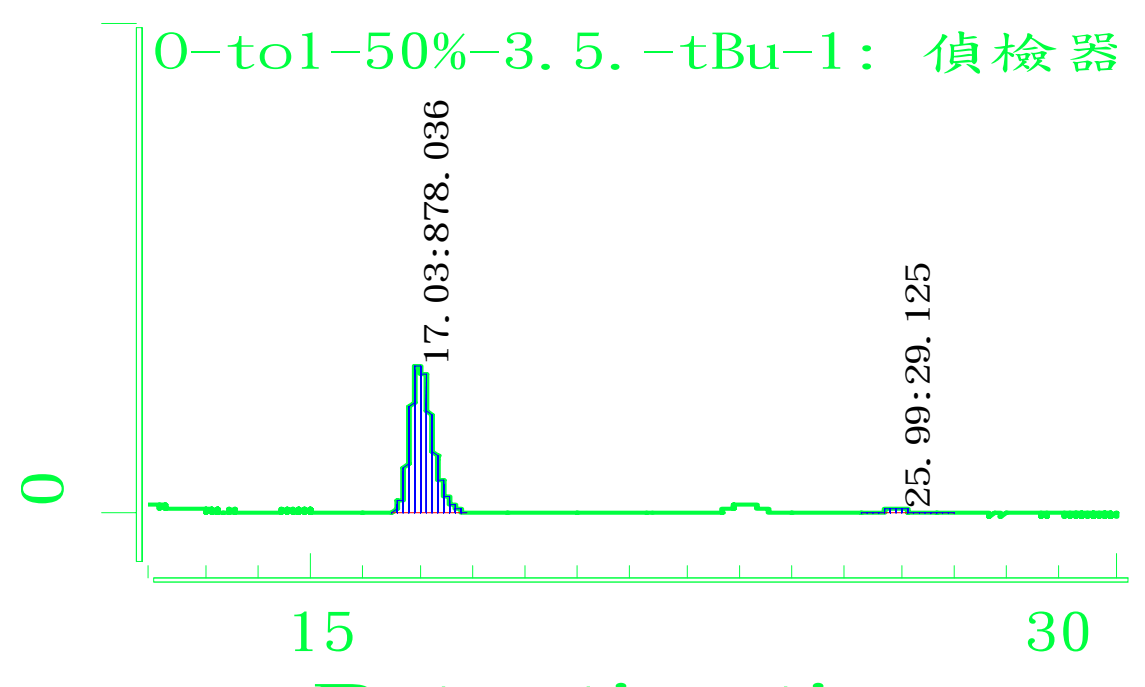

$\begin{array}{rrrr}\text { 滯留時間 } & \text { 面積 } & \text { 百分比 } & \text { 濃度 } \\ 17.026 & 878.04 & 96.79 & 0.00 \\ 25.990 & 29.12 & 3.21 & 0.00\end{array}$


Racemic 12: Chiralcel AD-H, ${ }^{\mathrm{i}} \mathrm{PrOH} / \mathrm{Hexane}=10 / 90$

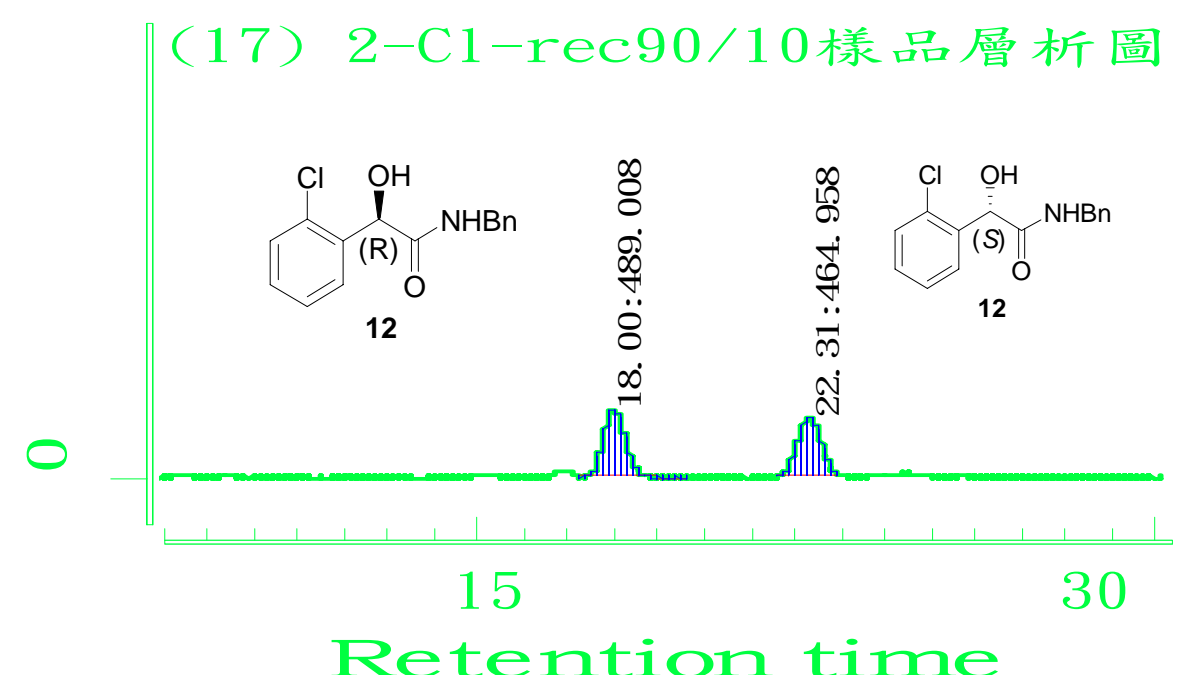

Retention tine

$\begin{array}{rrrr}\text { 滯留時間 } & \text { 面積 } & \text { 百分比 } & \text { 濃度 } \\ 17.998 & 489.01 & 51.26 & 0.00 \\ 22.314 & 464.96 & 48.74 & 0.00\end{array}$

Resolved

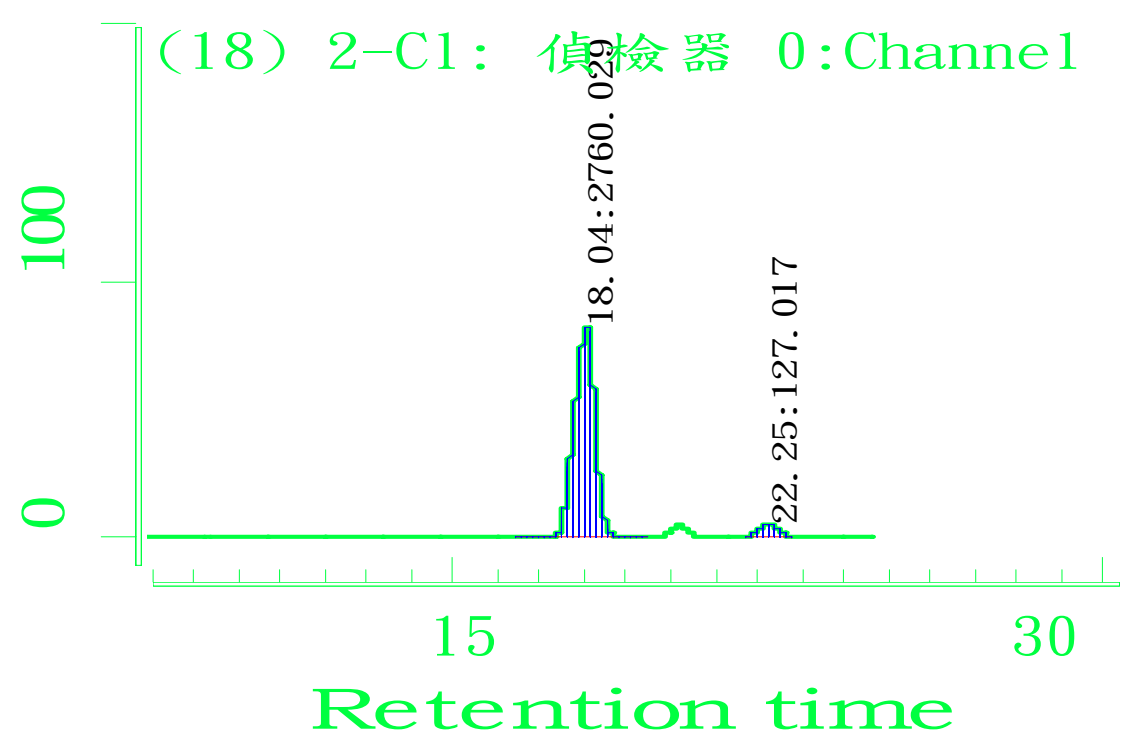

$\begin{array}{rrrr}\text { 滞留時間 } & \text { 面積 } & \text { 百分比 } & \text { 濃度 } \\ 18.041 & 2760.03 & 95.60 & 0.00 \\ 22.246 & 127.02 & 4.40 & 0.00\end{array}$




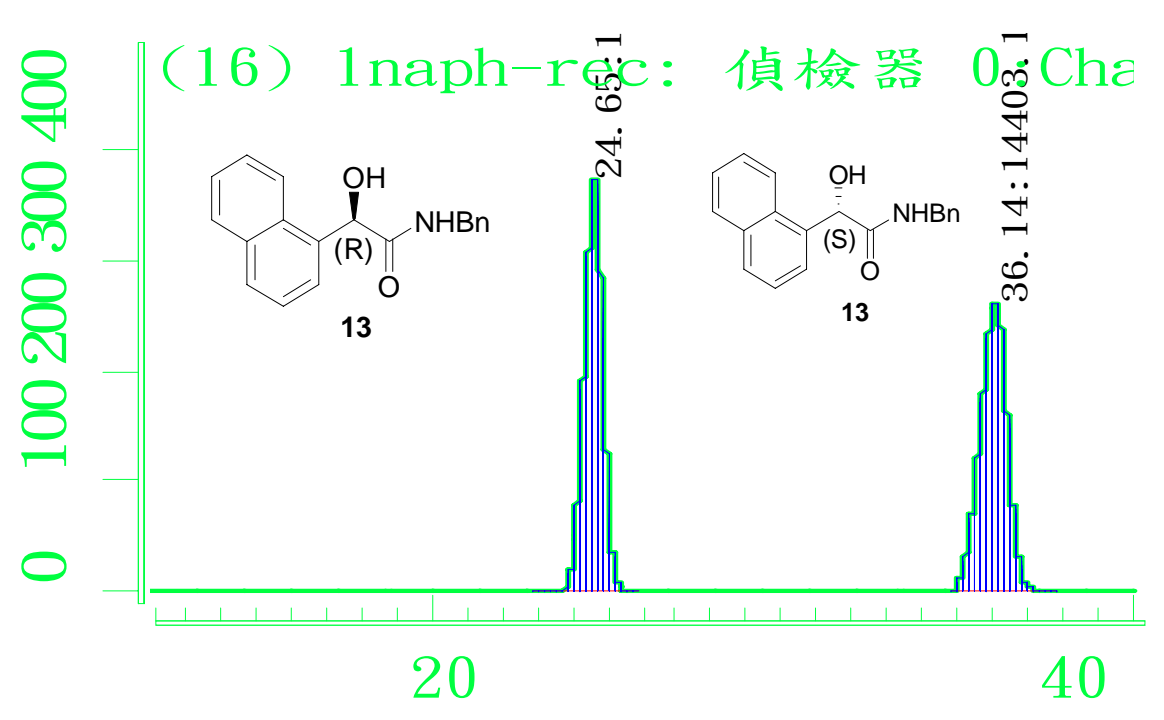

Retention time

$\begin{array}{rrrr}\text { 滯留時間 } & \text { 面積 } & \text { 百分比 } & \text { 濃度 } \\ 24.649 & 14550.98 & 50.26 & 0.00 \\ 36.143 & 14403.16 & 49.74 & 0.00\end{array}$

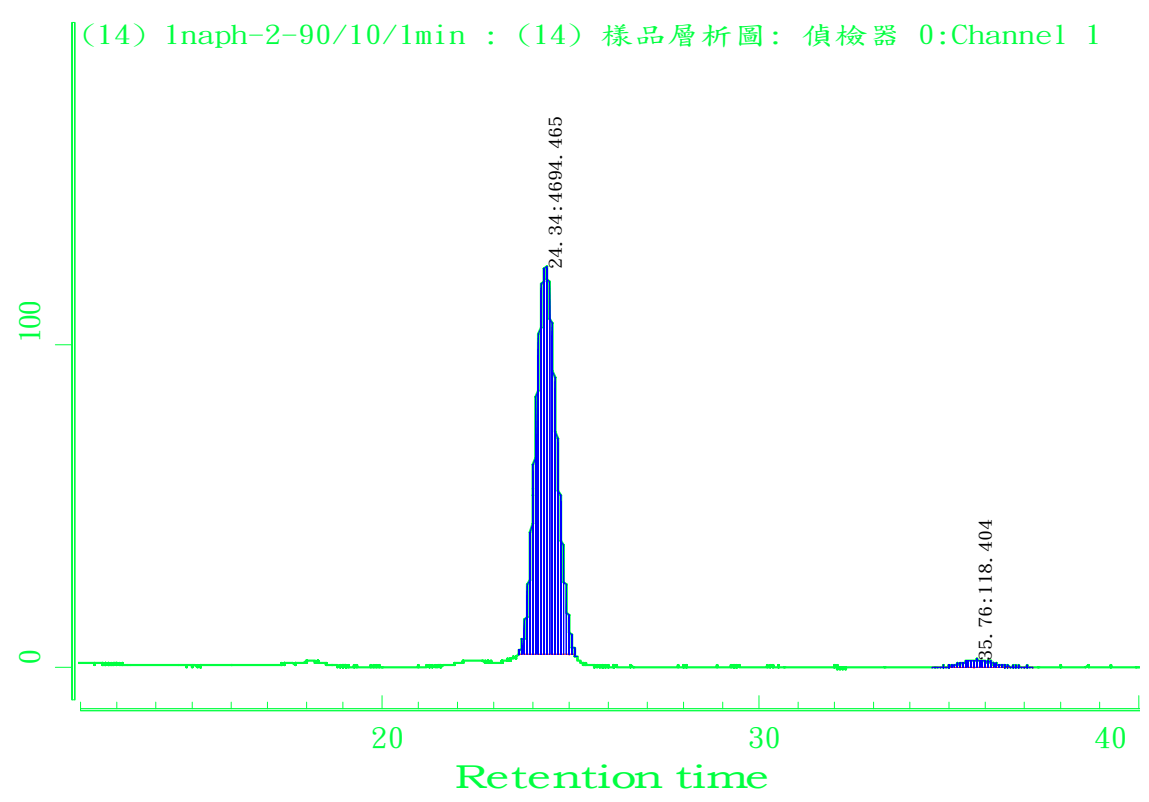

$\begin{array}{rrrr}\text { 滯留時間 } & \text { 面積 } & \text { 百分比 } & \text { 濃度 } \\ 24.343 & 4694.46 & 97.54 & 0.00 \\ 35.755 & 118.40 & 2.46 & 0.00\end{array}$




\section{Racemic 14: Chiralcel AD-H, ${ }^{\mathrm{i}} \mathrm{PrOH} / \mathrm{Hexane}=8 / 92$}

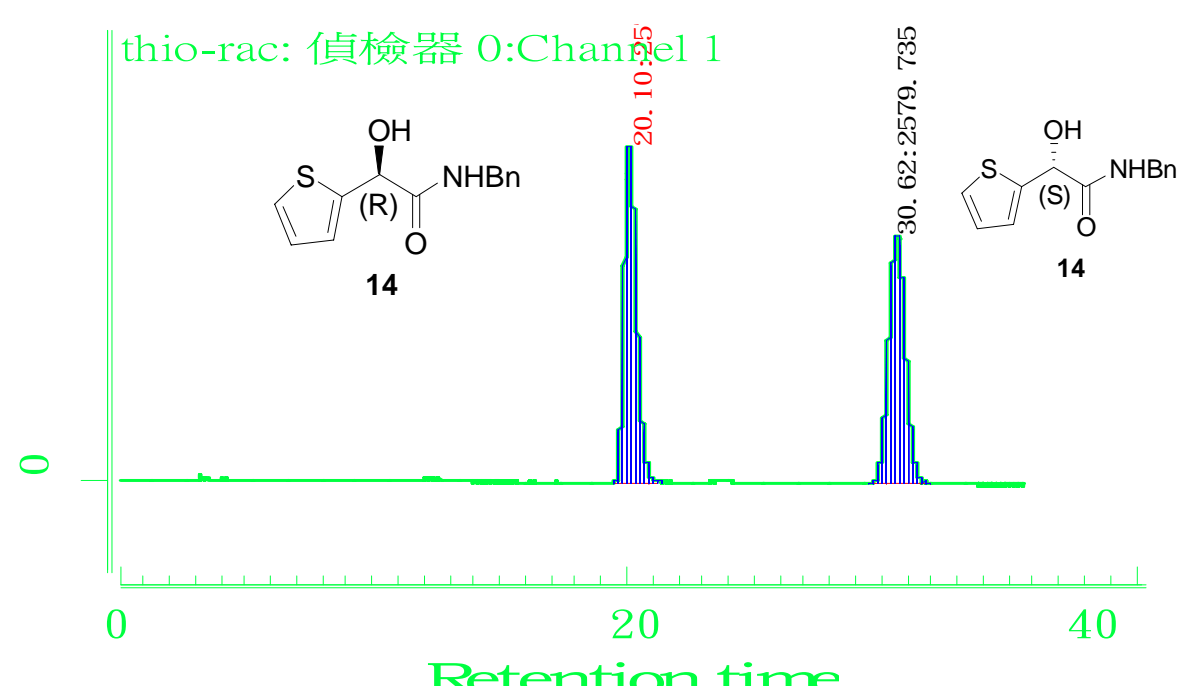

Retention time

$\begin{array}{rrrr}\text { 滯留時間 } & \text { 面積 } & \text { 百分比 } & \text { 濃度 } \\ 20.105 & 2579.85 & 50.00 & 0.00 \\ 30.622 & 2579.74 & 50.00 & 0.00\end{array}$

\section{Resolved}

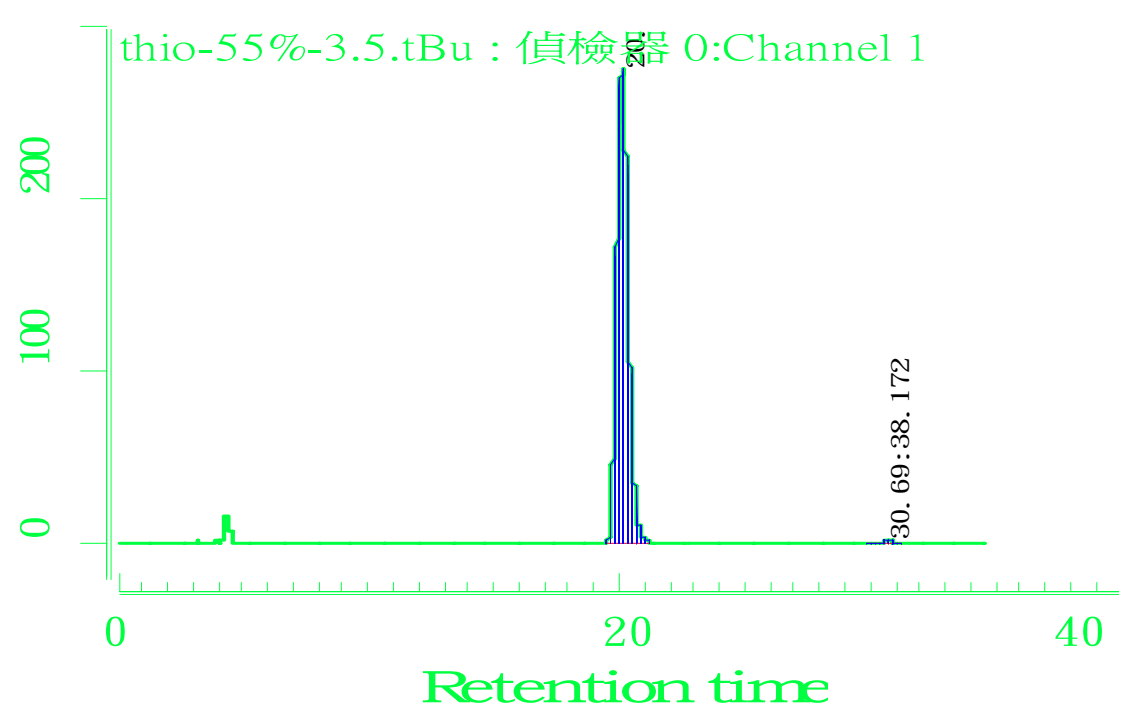

$\begin{array}{rrrr}\text { 滯留時間 } & \text { 面積 } & \text { 百分比 } & \text { 濃度 } \\ 20.131 & 9162.53 & 99.59 & 0.00 \\ 30.685 & 38.17 & 0.41 & 0.00\end{array}$


Racemic 15: Chiralcel AD-H, ${ }^{\mathrm{i}} \mathrm{PrOH} / \mathrm{Hexane}=8 / 92$

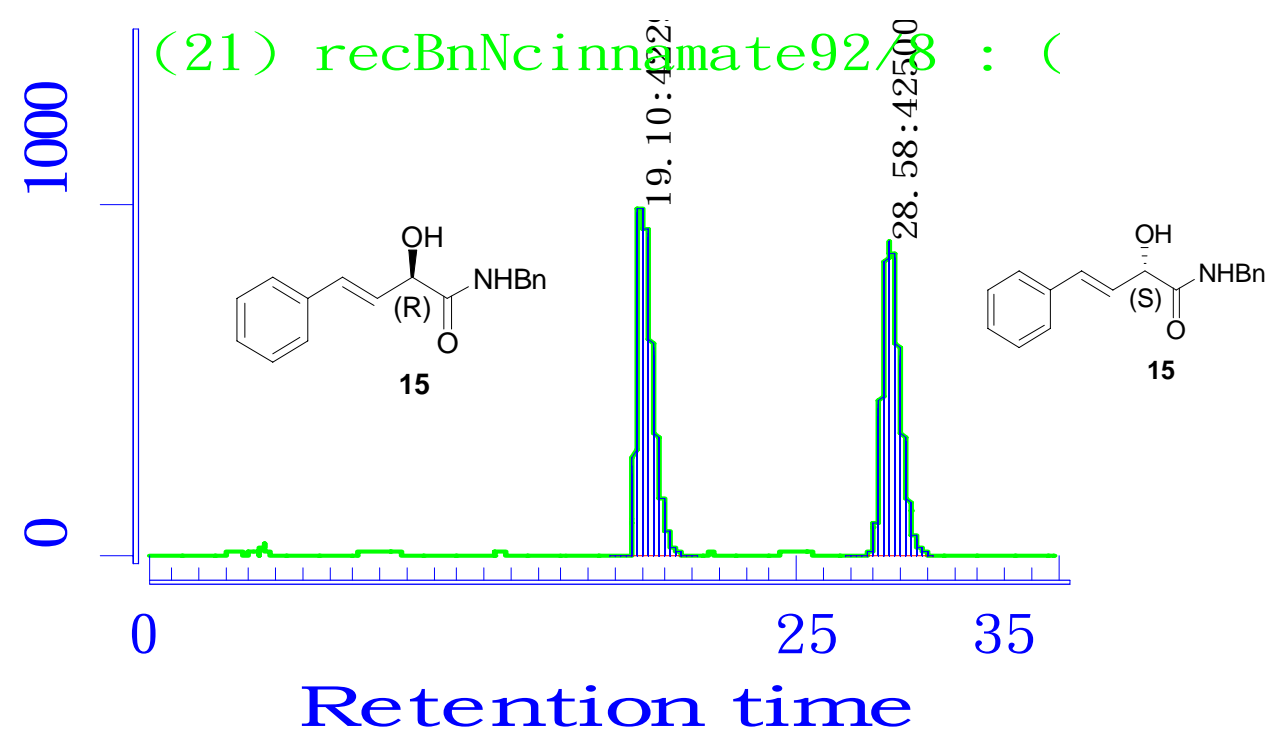

$\begin{array}{rrrr}\text { 滯留時間 } & \text { 面積 } & \text { 百分比 } & \text { 濃度 } \\ 19.099 & 42296.42 & 49.88 & 0.00 \\ 28.577 & 42500.96 & 50.12 & 0.00\end{array}$

Resolved

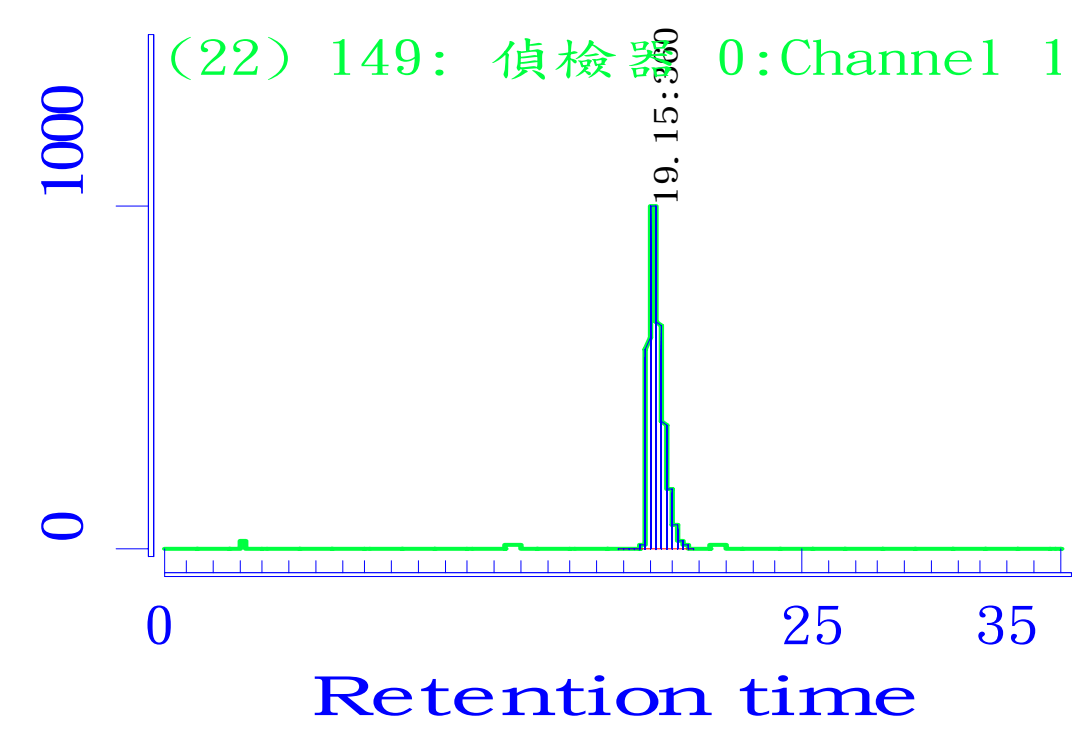

$\begin{array}{rrrr}\text { 滯留時間 } & \text { 面積 } & \text { 百分比 } & \text { 濃度 } \\ 19.150 & 36051.83 & 100.00 & 0.00\end{array}$


Racemic (94/6, AD-H) (16)

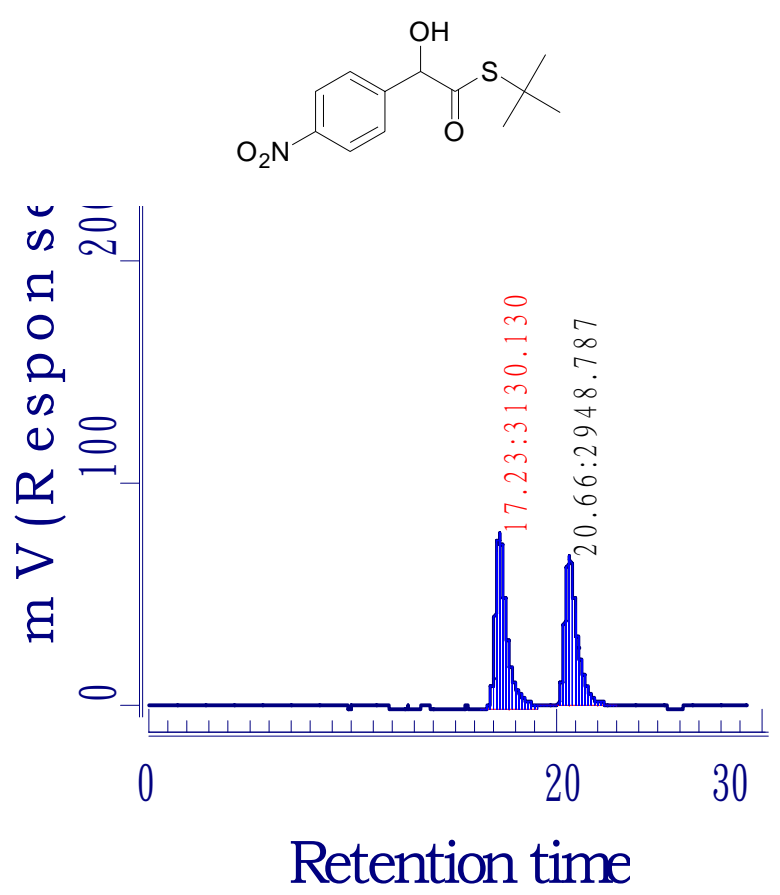

＃成分峰名稱

17.233

20.657

$\begin{array}{rr}\text { Area } & \text { 百分比 } \\ 3130.13 & 51.49 \\ 2948.79 & 48.51\end{array}$

Resolved

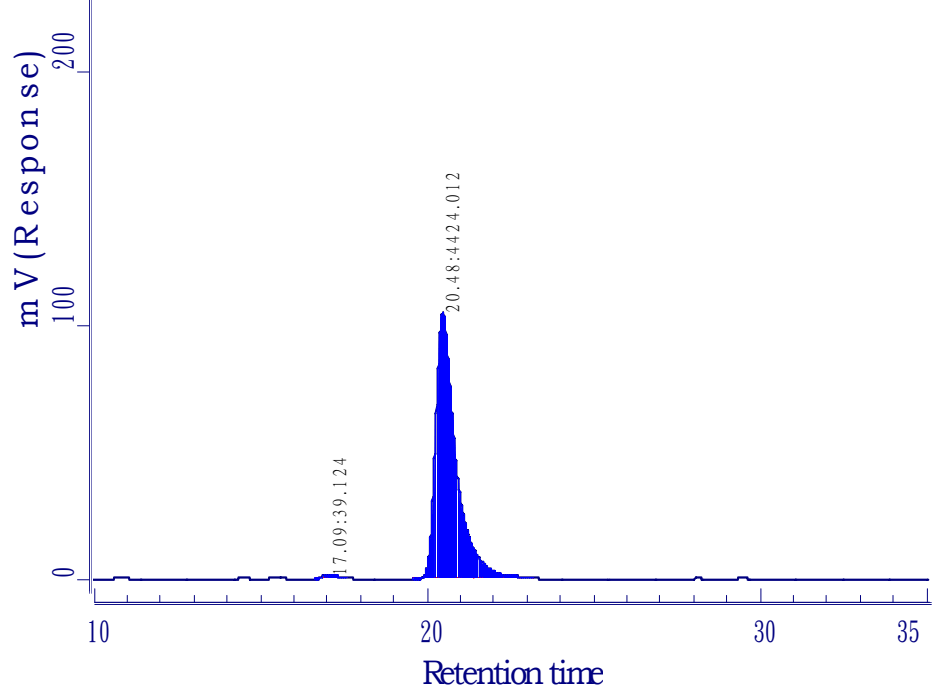

\# 成分峰名稱

$\begin{array}{rrrr}\text { 滯留時間 } & \text { Area } & \text { 百分比 } & \text { 濃度 } \\ 17.085 & 39.12 & 0.88 & 0.00 \\ 20.482 & 4424.01 & 99.12 & 0.00\end{array}$


Racemic (94/6, AD-H) (17)

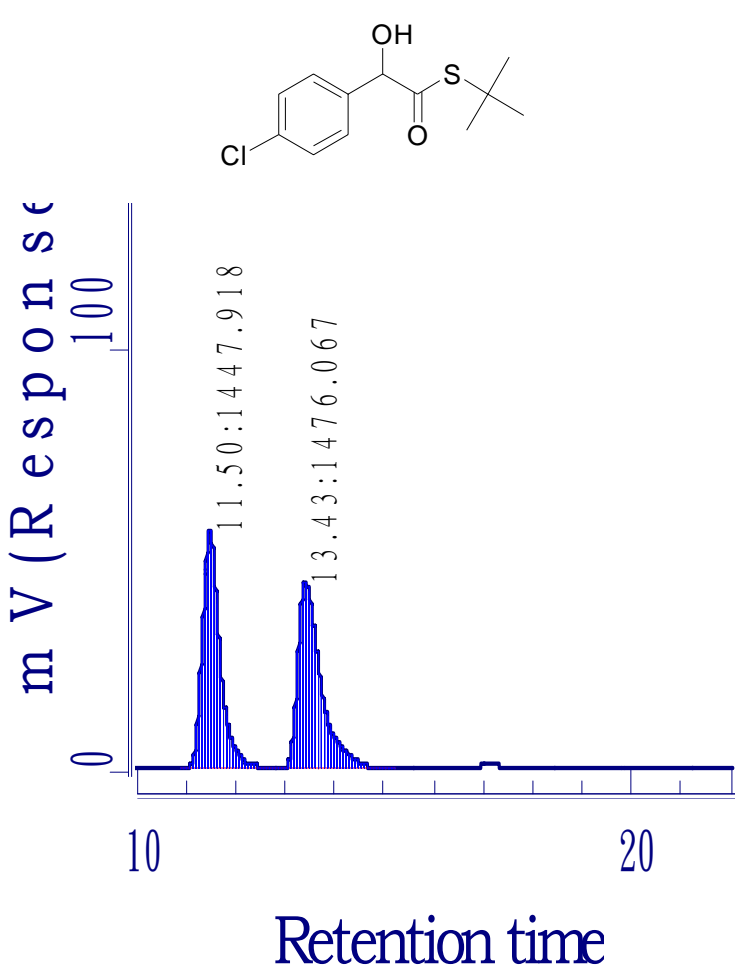

\begin{tabular}{|c|c|c|c|}
\hline 峰名稱 & 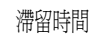 & Are & \\
\hline ( & 11.495 & 1447.9 & \\
\hline
\end{tabular}

Resolved

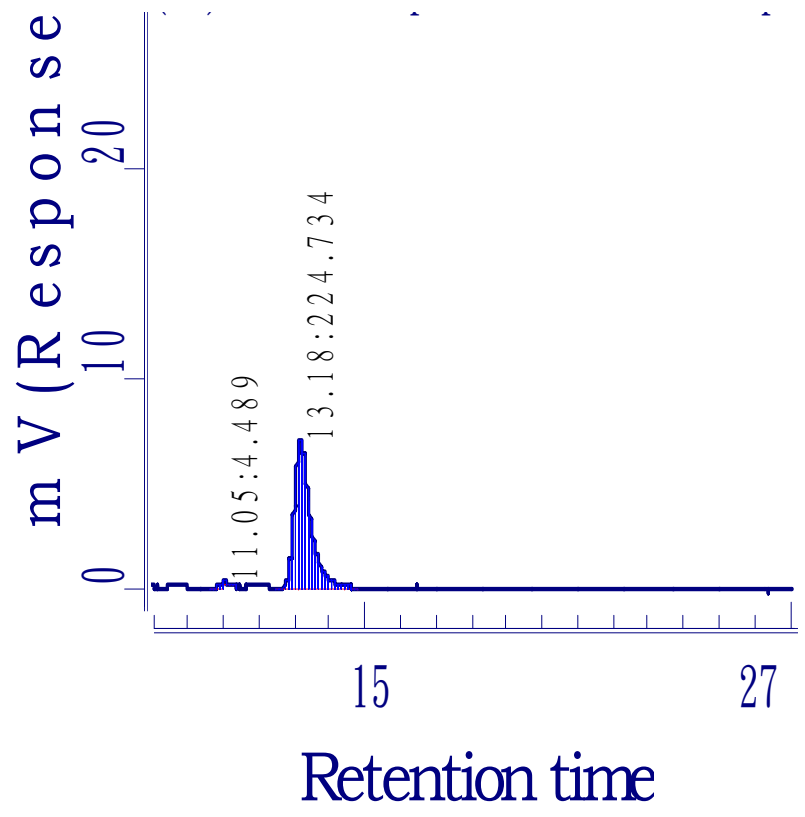

\#成分峰名稱

$\begin{array}{rrrr}\text { 滞留時間 } & \text { Area } & \text { 百分比 } & \text { 濃度 } \\ 11.051 & 4.49 & 1.96 & 0.00 \\ 13.179 & 224.73 & 98.04 & 0.00\end{array}$


Racemic (94/6, AD-H) (18)

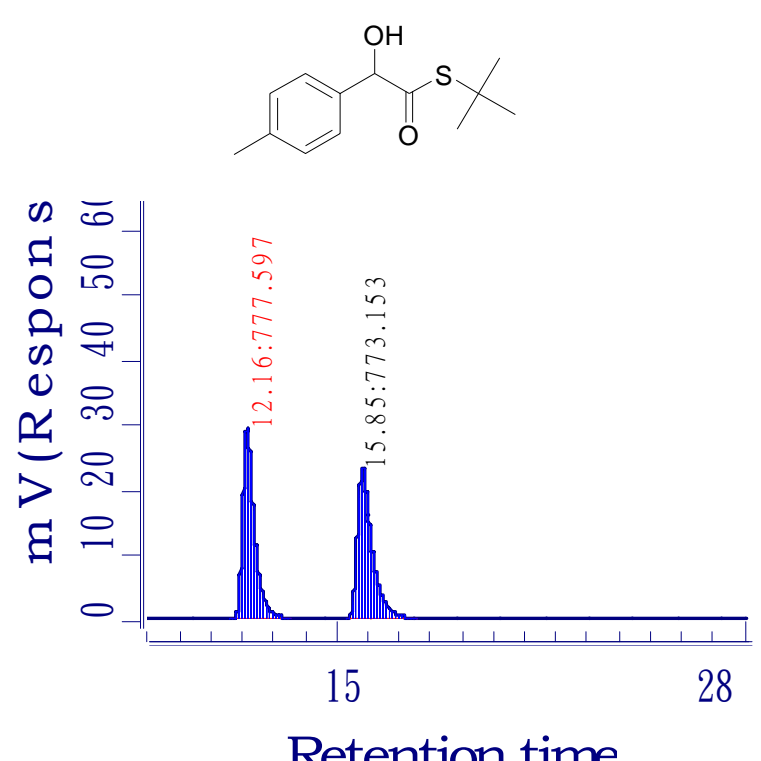

Resolved

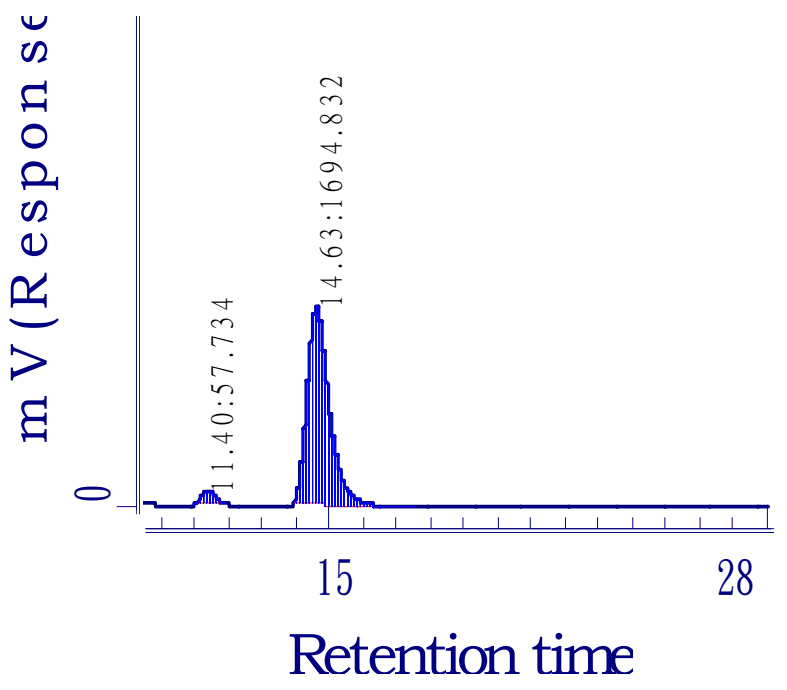

＃成分峰名稱

滯留時間

百分比

濃度

0.00 
Racemic (94/6, AD-H) (19)

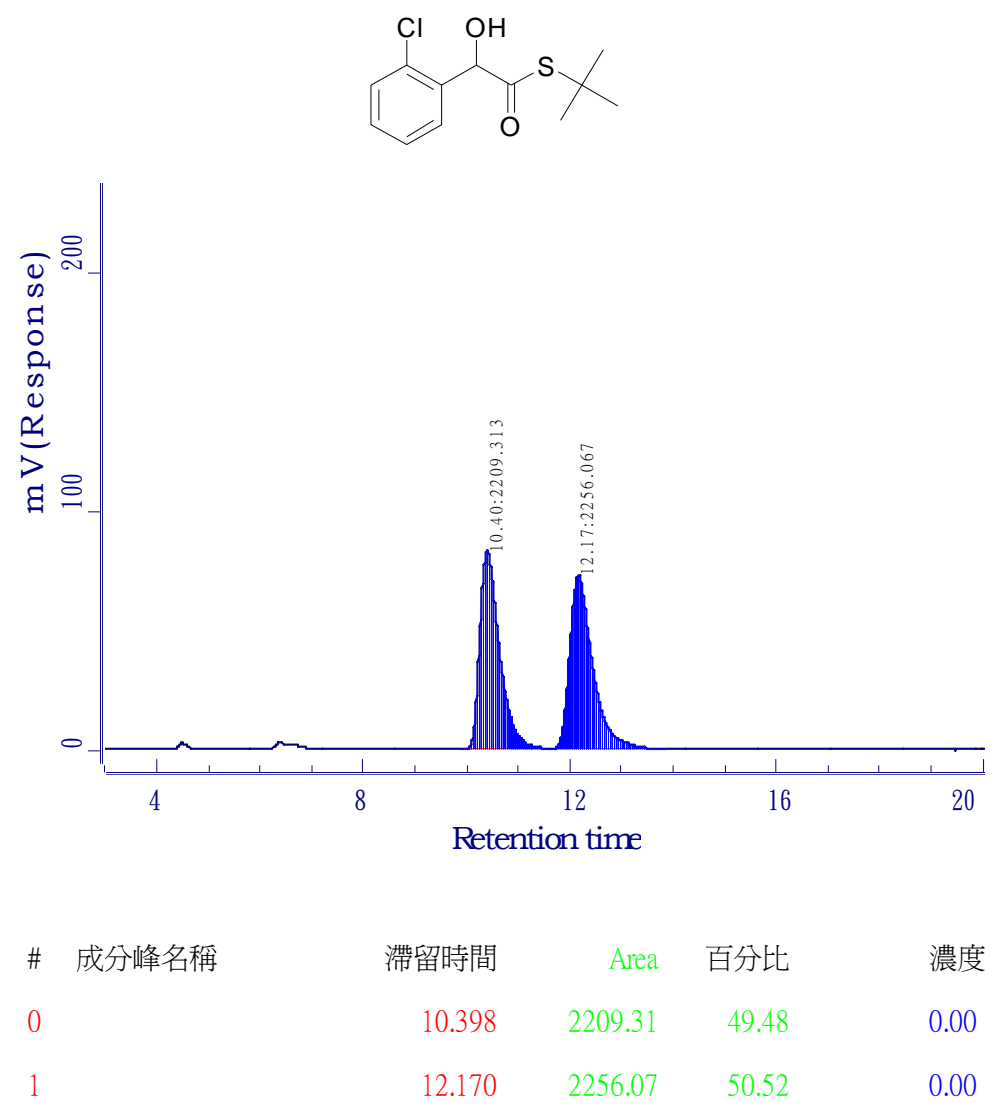

Resolved

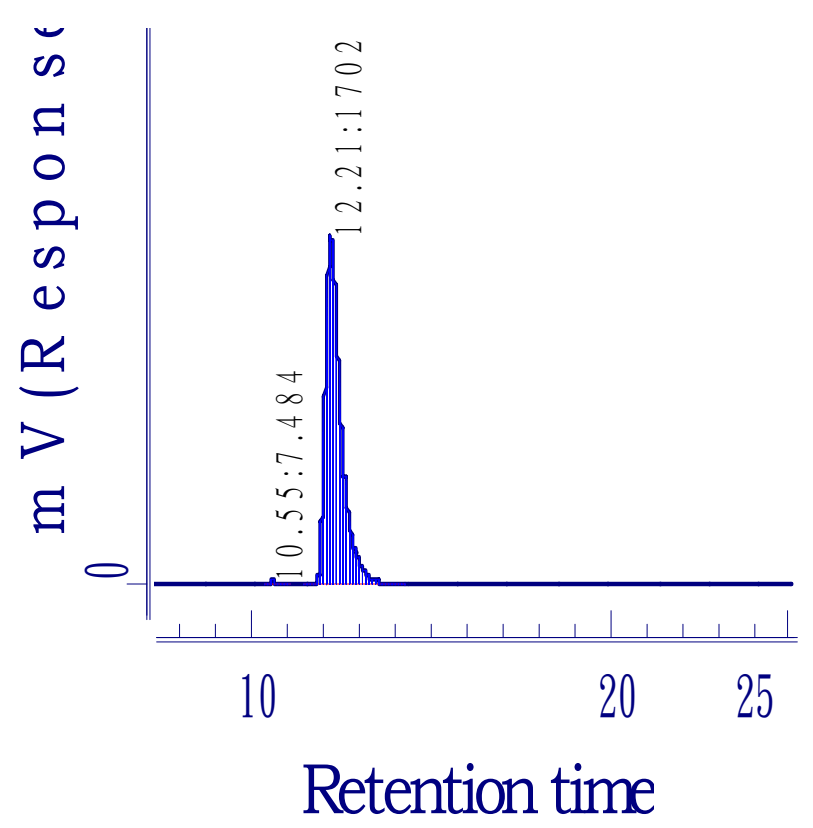

成分峰名稱

$\begin{array}{rrrr}\text { 帮留时間 } & \text { Area } & \text { 百分比 } & \text { 濃度 } \\ 10.551 & 7.48 & 0.44 & 0.00 \\ & & & \end{array}$


Racemic (94/6, AD-H) (20)
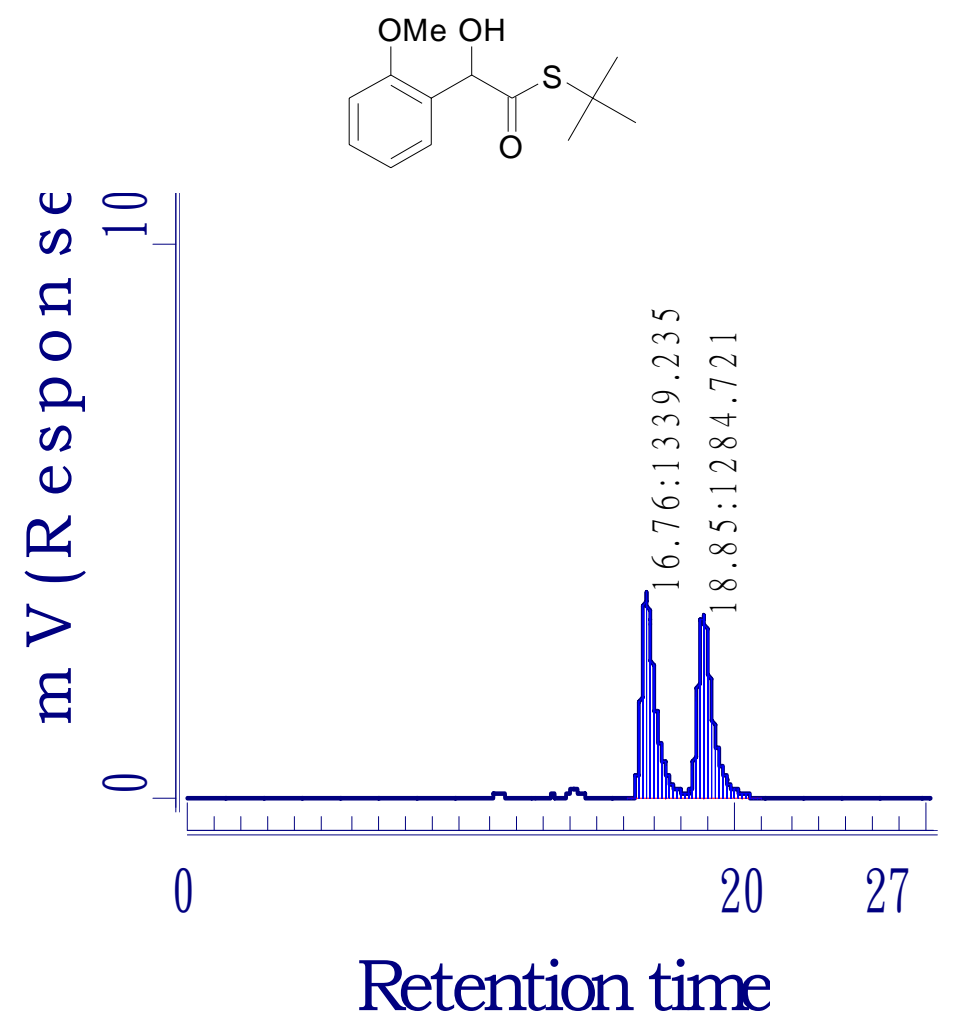

\# 成分峰名稱

$\begin{array}{rrrr}\text { 滯留時間 } & \text { Area } & \text { 百分比 } & \text { 濃度 } \\ 16.759 & 1339.24 & 51.04 & 0.00 \\ 18.847 & 1284.72 & 48.96 & 0.00\end{array}$

Resolved

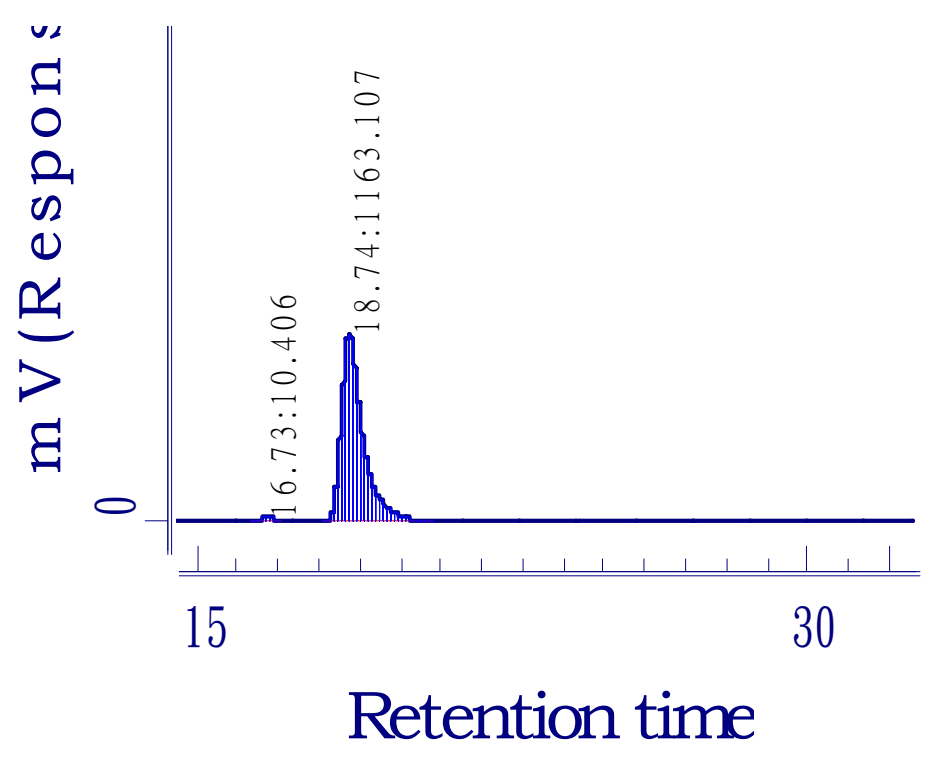

成分峰名稱

滯留時間

百分比

濃度

0

16.726

0.00

$\begin{array}{llll}18.744 & 1163.11 & 99.11 & 0.00\end{array}$ 
Racemic (96/4, AD-H) (21)
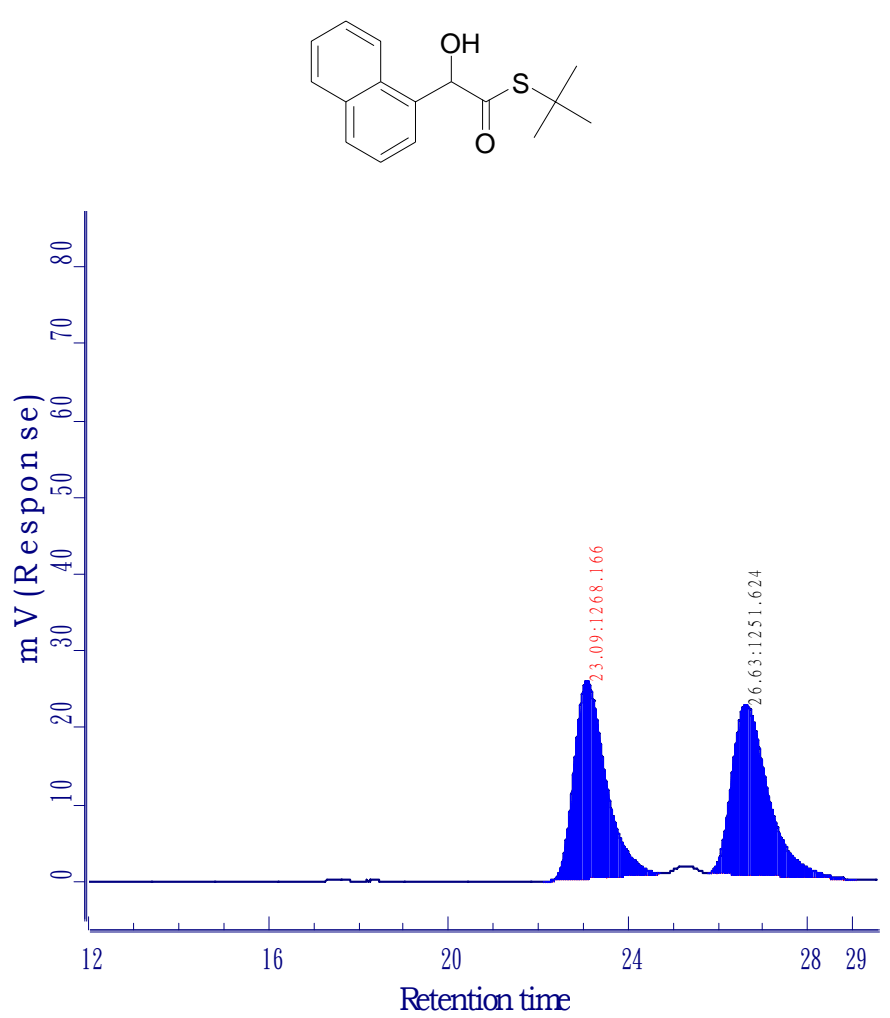

\begin{tabular}{|c|c|c|c|c|}
\hline 成分峰名稱 & 滯留時間 & Area & 百分比 & 濃度 \\
\hline & 23.090 & 1268.17 & 50.33 & 0.00 \\
\hline & 26.634 & 1251.62 & 49.67 & 0.00 \\
\hline
\end{tabular}

Resolved

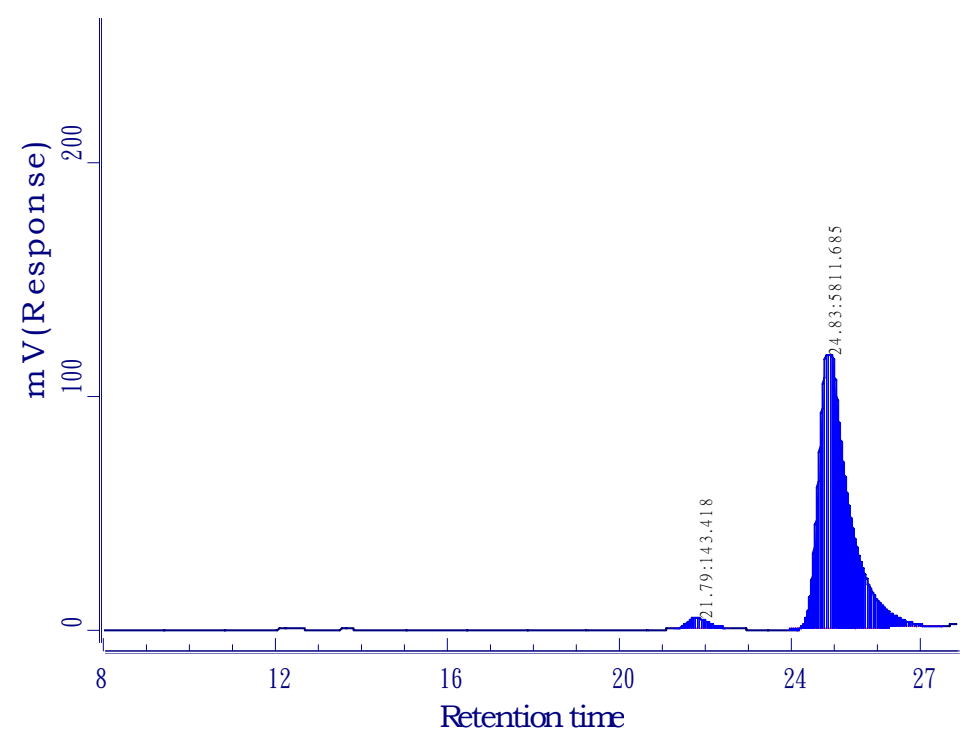

\begin{tabular}{|c|c|c|c|c|}
\hline 成分峰名稱 & 滯留時間 & Area & 百分比 & 濃度 \\
\hline & 21. 794 & 143.42 & 2. 41 & 0.00 \\
\hline & 24.831 & 5811.68 & 97.59 & 0.00 \\
\hline
\end{tabular}


Racemic (94/6, AD-H) (22)

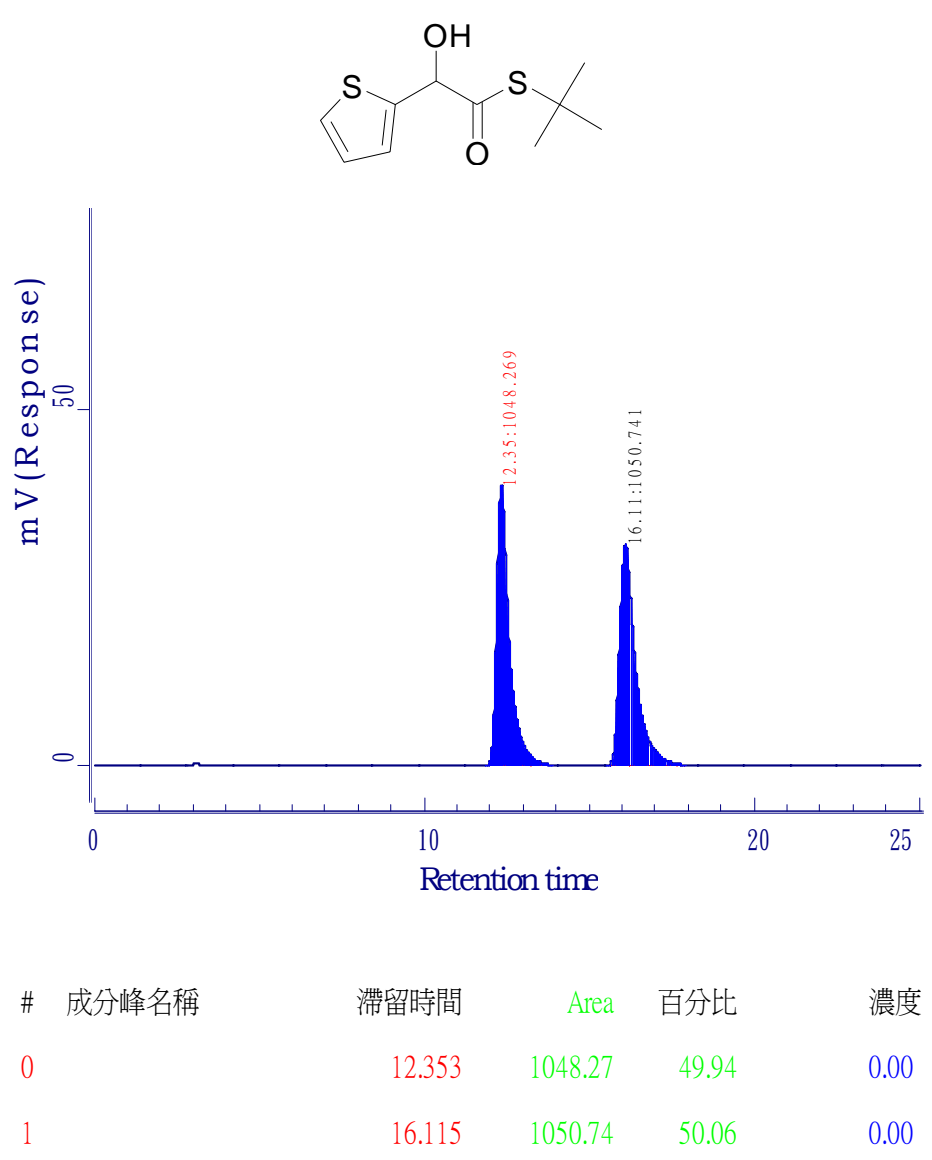

\section{Resolved}

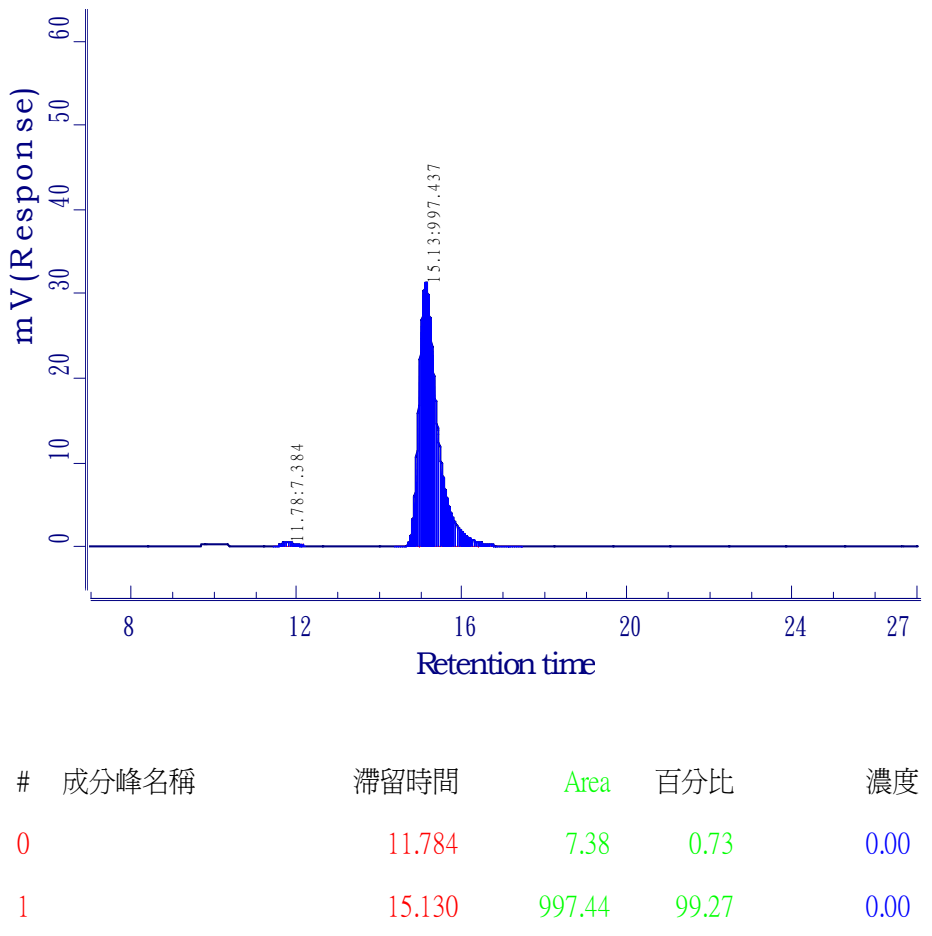


Racemic (98/2, AD-H) (23)

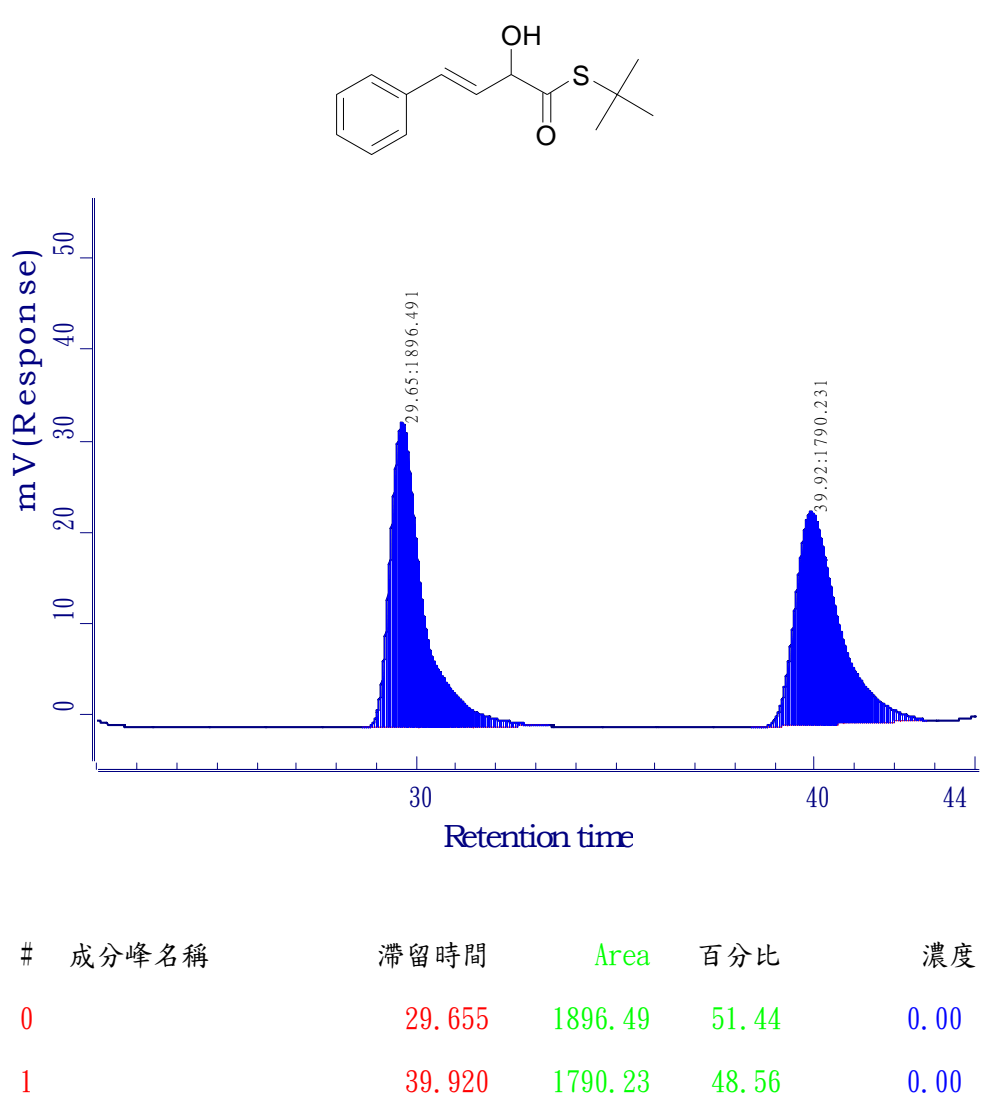

\section{Resolved}

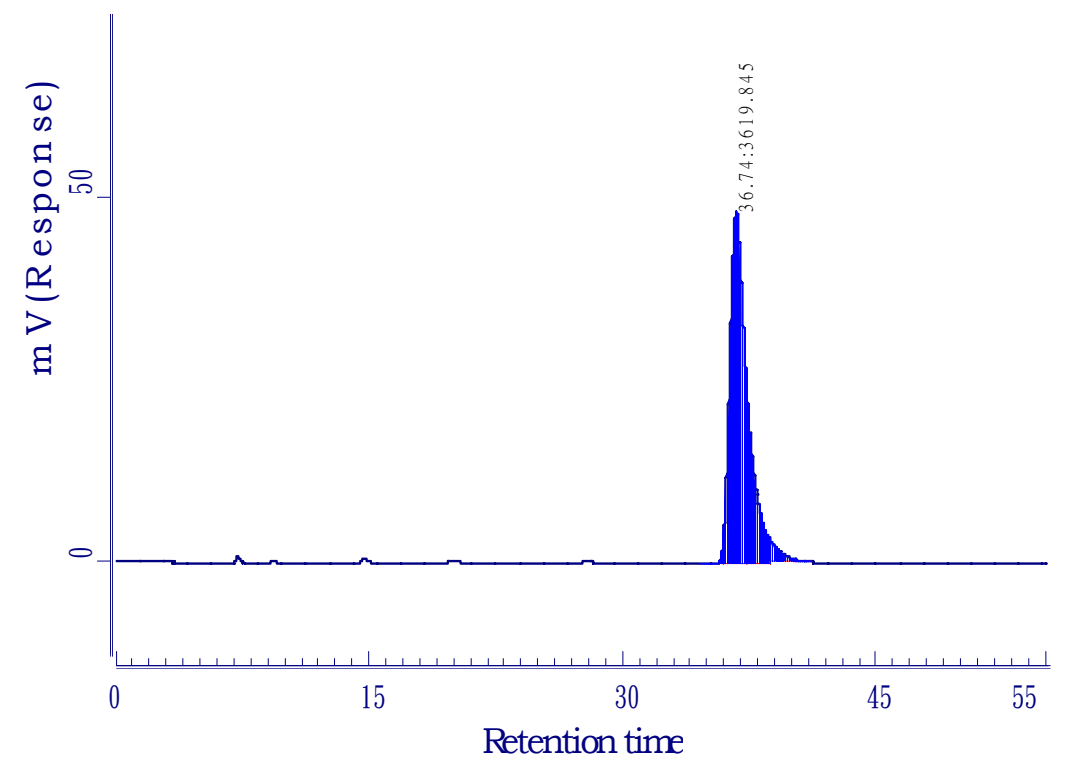

＃成分峰名稱

$\begin{array}{rrrr}\text { 滯留時間 } & \text { Area } & \text { 百分比 } & \text { 濃度 } \\ 36.744 & 3619.84 & 100.00 & 0.00\end{array}$


Racemic (98/2, AD-H) (24)

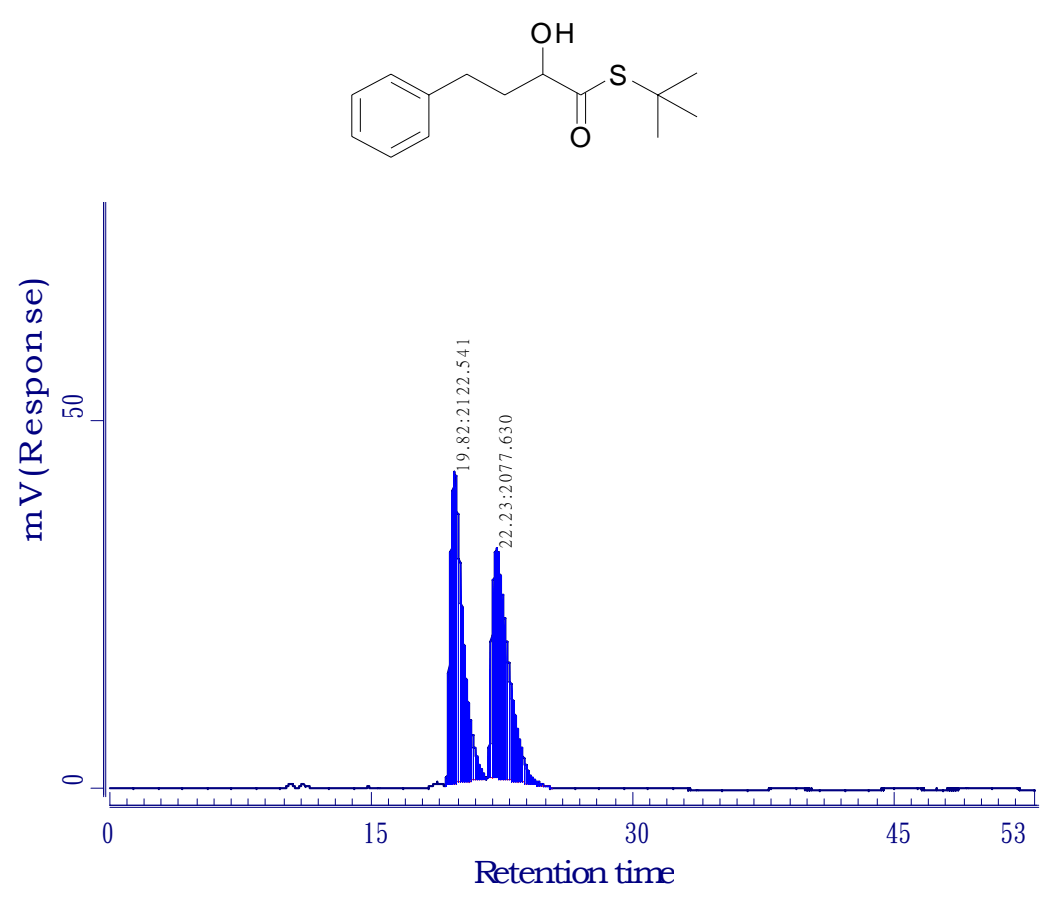

\begin{tabular}{|c|c|c|c|c|}
\hline 成分峰名稱 & 滯留時間 & Area & 百分比 & 濃度 \\
\hline 0 & 19.820 & 2122.54 & 50.53 & 0.00 \\
\hline & 22.225 & 2077.63 & 49.47 & 0.00 \\
\hline
\end{tabular}

Resolved

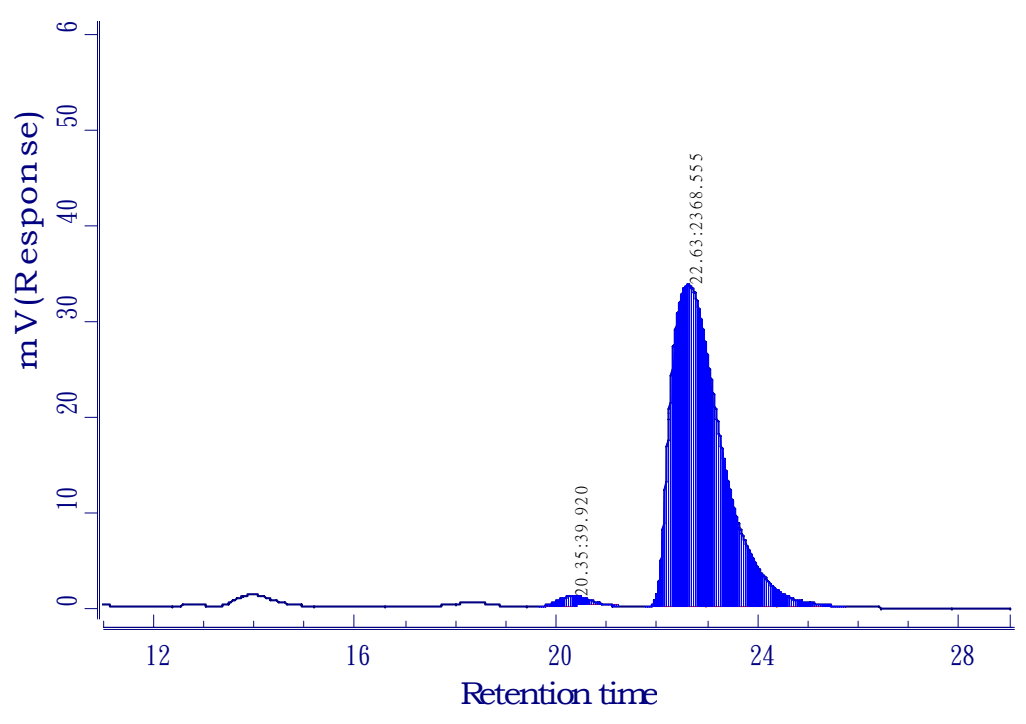

\# 成分峰名稱

$\begin{array}{rrrr}\text { 滯留時間 } & \text { Area } & \text { 百分比 } & \text { 濃度 } \\ 20.348 & 39.92 & 1.66 & 0.00 \\ 22.626 & 2368.55 & 98.34 & 0.00\end{array}$

